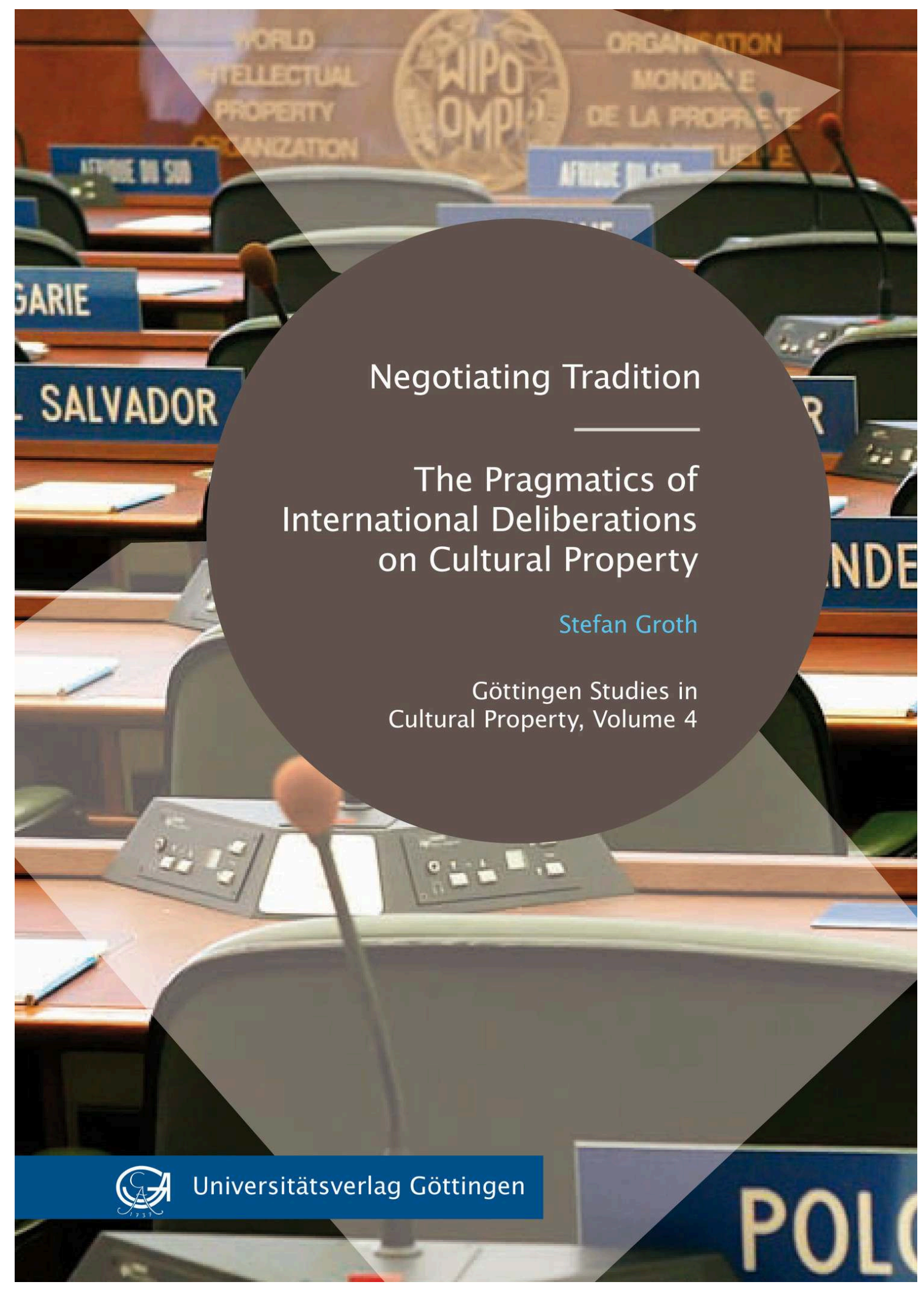




\section{Negotiating Tradition}

The Pragmatics of International Deliberations on Cultural Property

\section{Stefan Groth}

Publisher: Göttingen University Press

Year of publication: 2012

Published on OpenEdition Books: 12 April 2017

Serie: Göttingen Studies in Cultural Property

Electronic ISBN: 9782821875456

\section{Qboooks}

http://books.openedition.org

\section{Printed version}

ISBN: 9783863951009

Number of pages: 190

Electronic reference

GROTH, Stefan. Negotiating Tradition: The Pragmatics of International Deliberations on Cultural Property. New edition [online]. Göttingen: Göttingen University Press, 2012 (generated 10 September 2020). Available on the Internet: <http://books.openedition.org/gup/608>. ISBN: 9782821875456

(c) Göttingen University Press, 2012

Terms of use:

http://www.openedition.org/6540 


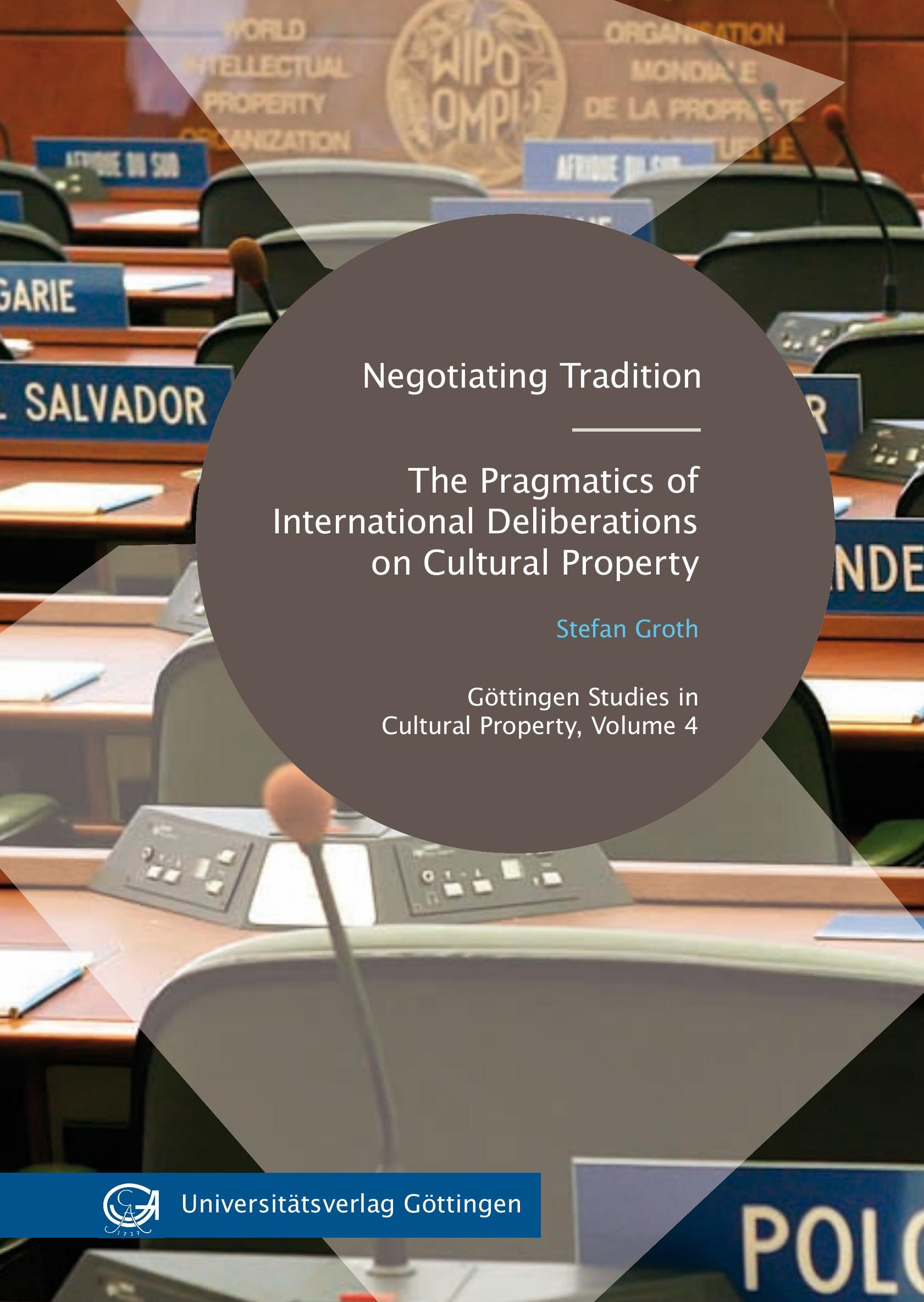



Stefan Groth

Negotiating Tradition

This work is licensed under the Creative Commons License 3.0 "by-nd", allowing you to download, distribute and print the document in a few copies for private or educational use, given that the document stays unchanged and the creator is mentioned. You are not allowed to sell copies of the free version.

SORIERIIGHIS RESERVED 
Published in 2012 by Universitätsverlag Göttingen as volume 4 in the series "Göttingen Studies in Cultural Property" 
Stefan Groth

Negotiating Tradition

The Pragmatics of International

Deliberations on Cultural Property

Göttingen Studies

in Cultural Property, Volume 4

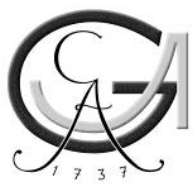

Universitätsverlag Göttingen

2012 


\section{Bibliographische Information der Deutschen Nationalbibliothek}

Die Deutsche Nationalbibliothek verzeichnet diese Publikation in der Deutschen Nationalbibliographie; detaillierte bibliographische Daten sind im Internet über $<$ http://dnb.ddb.de $>$ abrufbar.

Printed with funding from the German Research Foundation (Deutsche Forschungsgemeinschaft, DFG).

\section{Address of the Author}

Stefan Groth

e-mail: sgroth@gwdg.de

An earlier version of Chapter 5, "Multiperspectivity \& Differentiation", has been published as: Groth, S. (2010a) Perspectives of Differentiation: Negotiating Traditional Knowledge on the International Level.

Journal of Ethnology and Folkloristics 4(1): 7-24.

This work is protected by German Intellectual Property Right Law.

It is also available as an Open Access version through the publisher's homepage and the Online Catalogue of the State and University Library of Goettingen

(http://www.sub.uni-goettingen.de). Users of the free online version are invited to read, download and distribute it. Users may also print a small number for educational or private use. However they may not sell print versions of the online book.

Set and Layout: Stefan Groth

Cover: Stefan Groth

Cover Image: Ville Oksanen (http://www.flickr.com/photos/villoks/243843209/)

(C) 2012 Universitätsverlag Göttingen

http://univerlag.uni-goettingen.de

ISBN: 978-3-86395-100-9

ISSN: $2190-8672$ 
„Göttinger Studien zu Cultural Property“ / "Göttingen Studies in Cultural Property”

\section{Reihenherausgeber}

Regina Bendix

Kilian Bizer

Brigitta Hauser-Schäublin

Gerald Spindler

Peter-Tobias Stoll

\section{Editorial Board}

Andreas Busch, Göttingen

Rosemary Coombe, Toronto

Ejan Mackaay, Montreal

Dorothy Noyes, Columbus

Achim Spiller, Göttingen

Bernhard Tschofen, Tübingen

\section{Homepage}

http://gscp.cultural-property.org 

When a diplomat says yes, he means 'perhaps';

When he says perhaps, he means 'no';

When he says no, he is not a diplomat.

Voltaire, cited in Korta and Perry 2011 



\section{Table of Contents}

Preface: Up for Negotiation i

Donald L. Brenneis

Acknowledgements

List of Abbreviations vii

Note on Documents ix

1 Introduction 1

International Negotiations on Cultural Property and Language 1 The Debate on Cultural Property 8

Approach and Aims of this Study 10

2 On Terminology: Why Language Matters

Linguistic Anthropology 16

The Ethnography of Communication 18

Pragmatics \& Metapragmatics 21 
3 Negotiating Tradition on the Global Stage

February 200825

WIPO's Committee on Traditional Knowledge 31

Actors and Alliances 41

The Multiplicity of Communicative Events 49

4 The Pragmatics of Negotiating Cultural Property

The IGC as a Speech Community 56

Opening Statements 59

Language Politics in International Negotiations 68

Opening Statements as Framing Devices 75

Referential Frames of the IGC 107

Micro-Editing 136

5 Multiperspectivity \& Differentiation

Stigma 150

Potential 157

Right 160

Unity 161

Justice 164

6 Conclusion

Bibliography 


\title{
Preface: Up for Negotiation
}

\author{
Donald L. Brenneis
}

We often read of topics ranging from the details of local environmental planning to such potentially catastrophic issues as nuclear arms control being "under negotiation." We may have some sense of who the actors are, from local officials to diplomats, and we may have a sense that talk, document drafting, and the ongoing navigation of technical issues play major roles in such processes. At the same time, for most of us, and for many scholars, negotiation itself provides something of a terra incognita. A great deal has been written linking antecedent conditions, political and otherwise, to outcomes. Rarely, however, do we get a sense of the specific topics, styles, and strategies that figure in such crucial interstitial conversations, or of the complex intersections of talk, text, and decision-making they encompass.

Stefan Groth's book introduces us, with remarkable insight and ethnographic texture, to the often invoked but rarely deeply explored processes of diplomatic negotiation and into the routine communicative practices central to such activity. More specifically, he brings a breadth of methodological perspectives together to focus on the "negotiation of tradition" at the World Intellectual Property Organization (WIPO): How are decisions made, avoided, or transformed regarding the intellectual property status of "traditional knowledge and traditional cultural expressions/folklore" in this major transnational agency? And how do the specifics 
of quotidian talk in such cases speak to the larger political, commercial, and community interests that often figure more centrally in academic models of such decision-making?

In part his work reflects his strong core training in cultural anthropology, and especially his intense engagement with his colleagues in the Göttingen cultural property research group. It also draws very effectively upon recent social anthropological research on complex organizations. In particular, his research resonates with an emergent scholarly commitment to the ethnography of transnational regulatory regimes, whether in official institutions such as the United Nations and its derivative offices or in less formal nongovernmental organizations, parastatal agencies, and ad hoc entities. There is a great deal of significant and pathbreaking work being done in such sites, research that certainly challenges more orthodox organizational studies of these institutions. At the same time, Groth's work is utterly original, in part because of a third scholarly strain he brings to the work, that is, a thoroughgoing commitment to the detailed analysis of communicative practice at WIPO, a perspective grounded in linguistic anthropology.

The underlying argument of Groth's book begins with his emphasis on the signal importance of the sociolinguistic practices through which negotiation is constituted. He argues compellingly for the revelatory role of an "analytical triad" that brings together institutional ethnography, micro-linguistic analysis, and a thorough examination of those macro-processes, incentives, and constraints central to the broader context within which negotiation takes place. While institutional ethnographers often invoke the importance of negotiation, their analyses usually remain more or less metaphorical; they rarely grapple with the language through which such key work is accomplished. Groth not only focuses on such language, but he does so with a rich and subtle sense of the complex interworkings of meaning, style, social positioning, and indirection characteristic of such talk. This is linguistic anthropology of a very high order indeed. He has read widely, and with insight and imagination. His engagements with and uses of the work of key scholars are among the most lucid and generative I have read in recent years. Further, his innovative refigurings and applications of such concepts as shifters, "fractal recursivity" and social differentiation, intentionality, and speech community are sophisticated and fresh at the same time. In the United States, where there has been a much longer tradition of linguistic anthropology than in Europe, language specialists have only recently turned their research to the kinds of complex institutions at the heart of Groth's work: here again, his book provides a broadly applicable model for research of real value for scholars on both sides of the Atlantic.

Groth clearly demonstrates the value of his "analytical triad" through very detailed examinations of a range of recurrent communicative events and speech and documentary performances at WIPO. He argues that WIPO constitutes a "nascent" or always emergent speech community, that is, a collectivity that is always working to shape shared understandings not only of what their talk means but also 
of how it might most appropriately and effectively be pursued. In his examination of such occasions as formal meetings, hallway conversations, faction gatherings, and cocktail parties, Groth also explores such key features of WIPO talk as indirection, courteous evasion, and the multiple communicative manifestations of that constant tension between those delegations that use language to stall forward motion on binding legislation and those that work with equal energy to move towards regulatory closure. While his focus is on spoken language, he contextualizes all the talk within an extraordinary welter of well-explored documentary and documentmaking practices. In fact, the complex relationship between talk and text-making is at the political heart of WIPO's work. Groth is an acute and reflective ethnographer, one who consistently demonstrates great eyes and ears for telling materials. And he turns the materials of such ongoing interaction around to cast a strong and interpretively invaluable light on the broader life of the institution and of the transnational political economy in which it figures so significantly.

Negotiating Tradition illuminates or, perhaps more appropriately, makes audible the talk at the heart of WIPO. The talk itself, variously oblique, candid, and redundant, shaped by recurrent resistance and collaborative courtesies, is fascinating, and the interplay of formulaic performance and intentional strategy emerges elegantly. This is a compelling account in its own right, a clear-eyed, open eared, and subtle ethnography of a complex and consequential transnational forum. It also models, with principled imagination, what such an integrative perspective might bring to our own work. 



\section{Acknowledgements}

The group of people who have contributed to this book is larger than I expected when I first started working on it. In numerous conversations and encounters, they all helped shape it. All flaws, however, remain my own.

First and foremost, I would like to thank my thesis advisor Regina F. Bendix, without whom I would have never considered writing this book, let alone staying in academia. I feel truly fortunate to have had her generous support and invaluable insights on my work and on academia at large.

I am indebted to Don Brenneis for his generous support and expertise that enhanced this book significantly, both with regard to theory and methodology. I thank Charles L. Briggs and Clara Mantini-Briggs for their wonderful hospitality and the numerous conversations that made me more comfortable in viewing myself as a linguistic anthropologist.

My colleagues in the DFG Research Unit on Cultural Property provided me with assistance, commentary, and advice on numerous occasions. The interdisciplinary work environment was at times challenging, but very effective and stimulating.

Dorry Noyes is to be thanked for her always sharp comments and for helping me to think about the social dynamics of cultural property; Valdimar Hafstein for his insights on the entanglement of WIPO and UNESCO; Rosemary Coombe, Susan Gal, Anna Tsing and Christoph Bruman for their valuable comments on my Ph.D. project; and my student assistant Verena Pohl for her assistance. The DAAD is thanked for providing me with a fellowship for conducting Ph.D. research and the DFG funded the Research Unit on Cultural Property that this work stems from. Finally, I would like to thank John Bendix for his numerous valuable comments and careful final editing of the full manuscript. 



\section{List of Abbreviations}

$\begin{array}{ll}\text { AITIC } & \text { Agency for International Trade and Cooperation } \\ \text { AIWO } & \text { African Indigenous Women Organization } \\ \text { ARIPO } & \text { African Regional Intellectual Property Organization } \\ \text { ABS } & \text { Access and Benefit Sharing } \\ \text { AFS } & \text { American Folklore Society } \\ \text { BGC } & \text { Indigenous People (Bethechilokono) of Saint Lucia Governing } \\ \text { CBD } & \text { Council } \\ \text { CH-EM } & \text { Convention on Biological Diversity } \\ \text { CIEL } & \text { Ceniss Society for Ethnomusicology for International Environmental Law } \\ \text { DG } & \text { Director General } \\ \text { ECOSOC } & \text { United Nations Economic and Social Council } \\ \text { EOF } & \text { Expressions of Folklore } \\ \text { FAO } & \text { Food and Agricultural Organization } \\ \text { GA } & \text { General Assembly } \\ \text { GCCEI } & \text { Grand Council of the Crees (Eeyou Istchee) } \\ \text { GR } & \text { Genetic Resources } \\ \text { GIPID } & \text { Global Intellectual Property Issues Division } \\ \text { GRTKF } & \text { Genetic Resources, Traditional Knowledge and Folklore } \\ \text { ICC } & \text { International Chamber of Commerce } \\ \text { ICH } & \text { Intangible Cultural Heritage } \\ \text { ICTSD } & \text { International Center on Trade and Sustainable Development } \\ \text { IGC } & \text { Intergovernmental Committee on Intellectual Property and Ge- } \\ & \text { netic Resources, Traditional Knowledge and Traditional Cultural } \\ \text { IGO } & \text { Expressions } \\ & \text { Intergovernmental Organization }\end{array}$


International Institute for Environment and Development

ILC Indigenous and Local Communities

ILO International Labour Organization

IP

IPO Intellectual Property

KEI Intellectual Property Owners Association

NGO Knowledge Ecology International

OAPI

PCT

PIC Non-Governmental Organization

QMIPRI

African Intellectual Property Organization Patent Cooperation Treaty

RAIPON Prior-Informed Consent

SCP

SDS

Queen Mary Intellectual Property Research Institute

Russian Association of Indigenous Peoples of the North

Standing Committee on the Law of Patents

SIEF

RGCP

TCE

TK

Strategically-Deployable Shifter

TKDL

Societé Internationale d'Ethnologie et de Folklore

TRIPS

TWN

UN

Research Group on Cultural Property, Göttingen

Traditional Cultural Expressions

Traditional Knowledge

Traditional Knowledge Digital Library

Trade-Related Aspects of Intellectual Property

Third World Network

UNCTAD

UNDRIP

United Nations

UNESCO

UNHRC

UNPFII

UPOV

USPTO

$\mathrm{WCH}$

United Nations Conference on Trade and Development

United Nations Declaration on the Rights of Indigenous Peoples

United Nations Educational, Scientific and Cultural Organization

United Nations Human Rights Council

United Nations Permanent Forum on Indigenous Issues

International Union for the Protection of New Varieties of Plants

United States Patent and Trade-mark Office

WHO

World Cultural Heritage

World Health Organization

WIPO

WMO

World Intellectual Property Organization

WTI

World Meteorological Organization

World Trade Institute

WTO

World Trade Organization 


\section{Note on Documents}

The documents from the WIPO process used in this study are too numerous to be included in the bibliography with title and URL. For that reason, they are referred to by their document code. The documents themselves can be found on WIPO's homepage at:

\section{http:// www.wipo.int/meetings/en/topic.jsp?group_id=110}

They are listed by session, document code and title and by referencing convention. Thus, WIPO/IGC/GRTKF/10/4 refers to a document from the tenth session of WIPO's Intergovernmental Committee (IGC) on Intellectual Property and Genetic Resources, Traditional Knowledge and Folklore (GRTKF). 



\section{Introduction}

\section{International Negotiations on Cultural Property and Language}

In February 2008, I travelled to Geneva for my first fieldwork session at the World Intellectual Property Organization (WIPO), like the United Nations Educational, Scientific and Cultural Organization (UNESCO) or the World Health Organization (WHO) one of the United Nations' (UN) specialized agencies under the roof of its Economic and Social Council (ECOSOC). WIPO's activities "are dedicated to the use of intellectual property (patents, copyright, trademarks, designs, etc.) as a means of stimulating innovation and creativity", as the organization's website states. Since 2000, these activities include the Intergovernmental Committee on Intellectual Property and Genetic Resources, Traditional Knowledge and Traditional Cultural Expressions (henceforth the IGC). It brings together WIPO's 185 member states as well as hundreds of governmental and non-governmental organizations and deals with the protection of traditional knowledge and genetic resources by means of intellectual property (IP) law in its international dimension. As part of a larger interdisciplinary research unit based at the University of Göttingen, the main interest of my participation in the week-long twelfth session of the committee was in how and by what communicative means actors negotiate about the legal protection of cultural property on the international level. While one would expect actors in this forum to engage in controversial, yet substantive discussions about intellectual property legislation and cultural property, much of what could be observed were the exchange of very general views and deliberations about procedure: long-drawn statements were made with very few specific proposals, and under negotiation were rather questions about how to procede in the committee than how to reconcile the different interests of state actors and civil society representatives. At the end of the twelfth session in early 2008, the IGC's 
main decision was to compile two analyses identifying gaps in the intellectual property protection of traditional knowledge and traditional cultural expressions ${ }^{1}-\mathrm{a}$ late discussion of this basic task after almost seven years of committee meetings.

The WIPO IGC process is very slow, often with only minimal results at the end of a session (Bizer et al. 2010). Yet, in terms of efficiency, the negotiations at WIPO should not be judged only by their immediate outcomes. The IGC is a political arena where state actors represent their interest, and at times it is in the interest of actors to keep negotiations going without substantive results, be it to prevent issues from being negotiated elsewhere or to create political pressures in other fora. Being successful in negotiations can thus mean not to move forward, but just to continue discussions and maintain the status quo. Furthermore, the creation of alliances or an influence on the power dynamics of the international system can factor in the position of actors as well as domestic policy issues. WIPO is only one of many institutions on the international level dealing with cultural property questions. Their interplay and other political issues influence the position of state actors in this arena as well. Thus, what appears to be a slow and unproductive process at first sight might - under different evaluative criteria - be a successful and productive venture for some actors.

The purpose of this book is to clear up these evaluative criteria by looking at how actors in the IGC negotiate, and how their interests and intentions are reflected in their communicative actions. Its subject matter are the communicative strategies and patterns of international deliberations on cultural property. The book has two goals. The first is to clarify how participants in the IGC pursue their interests by means of language. How do they argue with each other, and how do they keep negotiations going despite extremely diverging interests? What communicative events and specifities characterize such negotiations? The second goal of this book is to apply the methods and theories of linguistic anthropology to the study of international negotiations. This book argues that they are an essential tool to understand the dynamics and presuppositions of deliberations on the international level.

This book focuses on the meetings of WIPO's IGC as well as on documentation about it and analyzes the communicative process involved in constituting cultural property at the international level. At the core stands an ethnography of communication of the committee meetings that take place twice a year in Geneva. The focus is on the communicative strategies that actors develop and employ, be these actors from the WIPO Secretariat, the national delegations, NGOs, indigenous groups or interest groups with observer status. Each of these actors has different motivations and very different levels of knowledge or information. What will be investigated in this book are the changing dynamics of communication and

\footnotetext{
${ }^{1}$ WIPO/GRTKF/IC/12/Decisions.
} 
the different forms of communication, including the ways in which IGC participants use language to defend their interests and strategies.

The debate on cultural property has grown very large, as has the literature on the subject. This debate is conducted under the heading of traditional knowledge, indigenous knowledge, traditional cultural expressions, expressions of folklore, ${ }^{2}$ tangible and intangible cultural heritage, or world heritage, and it takes place in conjunction with debates on biological diversity, biopiracy, ${ }^{3}$ genetic resources, land rights, human and cultural rights, safeguarding, repatriation or preservation efforts. The debate on cultural property is very difficult to define and delimit (Johler 2009: 44 ), and the very vagueness and versatility of the notion of cultural property and its many labels indicate its pervasiveness and ambiguity. Cases discussed under this label range from the unauthorized use of traditional knowledge for pharmaceutical research and development, the restitution of cultural artifacts in the context of museums to the commercialization of cultural expressions in the entertainment industry. Despite the extremely broad range of exemplary cases and their peculiarities there is a core issue under discussion that ties all these different fields and issues together. There are shared features and patterns that justify speaking of the debates about cultural property in terms of their commonality. The core issue of debates involves a cultural artifact or expression as the object of a process entailing:

(1) the justified or unjustified, recognized or unrecognized, attribution or appropriation of said artifact or expression to or by a group of social actors or an individual. These include but are not limited to nation-states, indigenous organizations and corporations in the entertainment or pharmaceutical sector; and

(2) decisions and judgements whether this artifact or expression should or can be conserved as is, altered in its qualities, or left to its own devices; thus, whether it should or can be safeguarded, protected or preserved, or not, or something in between. ${ }^{4}$

\footnotetext{
2 The term expressions of folklore (EOF) in this context stems from discussions between WIPO and UNESCO in 1982, resulting in "Model Provisions for National Laws on the Protection of Expressions of Folklore Against Illicit Exploitation and other Forms of Prejudicial Action" (1985). It specifically denotes folklore and expressions of folklore as part of national cultural heritage (cf. http://www. wipo.int/wipolex/en/text.jsp?file_id=184668, last accessed 07.01.2012).

3 See Dutfield 2004 for a definition: "The word 'biopiracy' [...] normally refers either to the unauthorized extraction of biological resources and/or associated traditional knowledge from developing countries, or to the patenting of spurious 'inventions' based on such knowledge or resources without compensation" (52).

${ }^{4}$ Folklorists have argued intensely against the stereotypical assumption that the perspective of the humanities - in particular, of folkloristics and anthropology - is to insist on collecting and preserving. See Noyes 2010, Hafstein 2009, Kirshenblatt-Gimblett 1998 for more balanced, reflexive and critical assessments of cultural property from a folkloristic viewpoint.
} 
While the first aspect concerns the relationship of the object - the cultural artifact or expression - to a social actor in terms of belonging or being attributed to someone, the second aspect looks at the qualities of this object and its actual and notional functions and values for a group of social actors via the discursive processes it is being referenced in. Thus, one aspect of the core issue in cultural property debates is the social attribution of meaning concerning the ownership of both material and immaterial culture in and across different groups of social actors. ${ }^{5}$ This relational dimension involves questions of temporality - how attributing practices evolve and change over time in the sense of the social biographies of artifacts and expressions - and space - how processes of spatial demarcation and distribution influence the ways in which these artifacts and expressions are perceived. A second aspect are the discursive processes and actions pertaining to the question how cultural artifacts and expressions are to be dealt with. Among these options are legal protection, physical preservation and conservation, archival documentation and commercialization, or taking no specific actions at all, acquiescing exploitation and decay of cultural property. These two aspects are hard to separate from one another: indeed, they act in mutually reinforcing manner. ${ }^{6}$ Most studies of cultural property issues thus deal with both aspects, some focussing more on property relations, others more on questions of safeguarding, protection and preservation. There are also studies which primarily examine the relation between the two.

Missing in these wide-ranging debates is an analysis of how different perceptions of cultural property, including assessments of their social, political and economical potentials and values, come together in international negotiations. On the international level, there is a large and growing number of committees, agencies and other bodies dealing with questions of cultural property. It is on this level that nation states, non-governmental organizations (NGOs) and other actors deliberate on the aspects of owning and handling cultural property. Guidelines, conventions or treaties regulating the use and ownership of cultural artifacts and expressions are generated, connections to other issues are drawn and political coalitions are formed. This international dimension has - for good reason - gained increasing

\footnotetext{
5 The distinction between material and immaterial or tangible and intangible culture is not to be understood as a strict dichotomy. This perspective has been criticized at length, as intangible culture almost always includes some kind of materialized form and vice versa (see Shand 2002: 60).

6 Property primarily has a social dimension that demarcates what is mine from what is yours - without a demarcation of desire for a thing there is no property to start with, so that property is less the relationship between people and things, but rather the relationship of people with people by means of things (Kojève 1969: 40). To draw the distinction between oneself and an object, one needs to be self-conscious, but in order for this self-consciousness to exist, one needs to recognize the existence of other self-conscious subjects. Property in this sense is always social, and the question is less whether property relations influence assessments of cultural artifacts or expressions, but how the constellations between them are framed. See also Hann's notion of the "embeddedness of property" (Hann 1998), Kasten 2002a, 2002b, 2004, and Kuutma 2009a for a discussion of the social function of cultural property.
} 
attention in the course of the last years (Dutfield and Suthersanen 2008), ${ }^{7}$ as instruments and frameworks stemming from its activities significantly shape national policies and local practices (Bendix et al. 2012).

Yet, aspects of how and by what specific communicative means actors negotiate on this level have largely been neglected. This is somewhat surprising, given that the communicative interactions in international negotiations do not only provide information about the emergence and proliferation of arguments, rhetorics, and registers related to cultural property issues, but also permit valuable insights into actors' positions, strategies and alliances. Furthermore, they significantly influence local and national practices and views related to cultural property debates. The discussions and decisions on the ownership and handling of cultural artifacts and expressions in supranational fora considerably shape heritage and property practices that have been the subject of scholarship from anthropology and folkloristics in the last decades. An analysis of only contents and outcomes is not sufficient for an understanding of the processes constituting the "metacultural" fields of cultural property and cultural heritage (Kirshenblatt-Gimblett 1998). Essential for international negotiations on cultural property, but also on other issues, is that people participate in communicative interactions. On the one hand, what can be gained from a deep analysis of the communicative patterns and strategies that actors engage in - the entailing text and talk of negotiations - is a better understanding of the process itself: how do different actors argue, what kind of strategies and rhetorics do they use, to which instruments and institutions do they refer, and in what way do actors react to each other? In other words, an analysis of communicative interactions contributes to the question of how international negotiations work. On the other hand, the analytic inclusion of sociolinguistic practices allows insights into positions, strategies, and perspectives pertaining to cultural property. By looking at not only what actors say, but also at how and in what contexts they do so, it is possible to make more accurate statements about their positions and perceptions in cultural property debates. As these communicative interactions influence outcomes considerably, an approach like this is in the last instance not only beneficial for an understanding of specific negotiations, but also for the analysis of broader cultural property issues.

There is thus much reason to analyze the specific ways various actors debate about these issues, and this study will do so by looking at an intergovernmental committee dealing with intellectual property (IP) and traditional knowledge (TK), traditional cultural expressions (TCEs) and genetic resources (GRs). It focuses on language and the communicative processes of international negotiations on cul-

\footnotetext{
${ }^{7}$ However, despite anthropologists and folklorists being involved in international fora as observers or advisers, most of the relevant literature comes from legal studies (Graber 2007, Porsdam 2007, Macmillan 2008, Coombe 1998, 2003; see Coombe 2009b for an extended literature review). A number of anthropological projects are taking a closer look at processes of global governance and cultural property, but as of this writing, no published papers or monographs have resulted.
} 
tural property. More precisely, it is about the various ways that the usage of language influences settings in which actors from a multitude of social and professional backgrounds engage in deliberations about cultural property. My focus will be on the substantive as well as strategic bearing of linguistic utterances and their ideological content ${ }^{8}$ in a contested field in which slight changes in text and talk can at times have severe implications for its participants: in treaty language, replacing a "should" for a "shall" can cause conflict.

The main claim of this study is that there is a specific dynamic repertoire of linguistic conduct and strategies in such international settings, and that the analysis of this repertoire can help to clarify different perspectives on cultural property, both for scholars and practitioners. By analyzing the communicative interactions in negotiations, strategies pertaining to cultural property as well as relations to other issues and fora can be pointed out. Furthermore, by combining "conventional" ethnography with linguistic analysis and a study of the macro-processes central to the broader context within which negotiations take place, perceptions of cultural property and related issues can be grasped in their richness and complexity. While cultural property negotiations have their specificity which will come to light in the course of the study, the focus on repertoires of linguistic conduct and strategy is essential for contributing to the understanding of all international negotiations. By illustrating the merits of this analytic perspective, the study thus hopes to encourage its inclusion in studies of international negotiations as well as international organizations more generally.

The functions of language and communicative practices in international negotiations are manifold, and both language and communicative practices are of central importance to the study of international negotiations on cultural property. Language in this context of international negotiations is used to purposefully differentiate positions and to create pressure on actors that do not share the same view or strategy. It is used to stall or speed up the proceedings of an international body, to build alliances and to frame the issues under negotiation as connected or disconnected from other contexts. It is used to protest, to shed light on complex situations - or to obscure them. Language is used to render question of social justice technical and to translate issues of morality into the language of a given body of law. Language is used to bargain, to barter and to block, and at times, it is also used to reach an understanding.

International negotiations can be seen as communicative events in which actors negotiate meaning with a view to achieve their goals: decisions are drafted, revised and discussed to be precise at one point and vague at another; oral and written statements are translated and checked for accuracy and mistakes; different termi-

\footnotetext{
${ }^{8}$ In this context, ideologies are understood as socially shared belief systems with axiomatic qualities and high stability (van Dijk 2006: 116; see also van Dijk 1998).
} 
nology that needs to be understood and employed is attached to different fora and issues; communicative styles vary from venue to venue and conflicting language ideologies ${ }^{9}$ are in place. While many of these aspects are applicable to all international negotiations as well as other types of deliberations, their specific composition differs from forum to forum. For example, the ambiguity of terminology or sets of communicative strategies are shared by different settings. But regarding the substance and topic, their form shifts depending on contextual variables like the composition of actors and issues. While the specific substance of negotiations is of importance to the analysis and shapes communicative interactions, elementary communicative patterns and strategies are applicable to other fora as well. This study of international negotiations on TK, TCEs and GR is thus primarily relevant to debates on cultural property. But as regards sociolinguistic practices and pragmatic strategies, it applies to a much broader range of settings.

As early as 1982, the United Nations Educational, Scientific and Cultural Organization (UNESCO) and the World Intellectual Property Organization (WIPO) cooperated in drafting "Model Provisions for National Laws on the Protection of Expressions of Folklore Against Illicit Exploitation and Other Prejudicial Action". This can be viewed as the starting point for a series of international ventures and fora occupied with questions of intangible cultural property. Over time, such international negotiations have increased in importance and have drawn attention in domestic and international policy debates. This has been most notably evident in the context of UNESCO and the branches within it that are concerned with questions of cultural property and cultural heritage. ${ }^{10}$

Negotiations at WIPO, on the other hand, are less visible, with little news coverage of its proceedings as substantial results have yet to be produced by the committee dealing with cultural property. WIPO negotiations are moreover entangled with a number of other fora, for instance the Convention on Biological Diversity (CBD), the World Trade Organization (WTO), the International Labour Organization (ILO) and of course with UNESCO itself. The interlocking negotia-

${ }^{9}$ I use the notion of language ideology as defined in Errington 2001: "Language ideology refers to the situated, partial, and interested character of conceptions and uses of language. It covers a wide range of concerns: the differential openness of language structure for metalinguistic objectification; the ways metalinguistic discourses can mediate social interests; the 'naturalization' of social differences through construals of language as embodying identity and community. In these and other ways, 'language ideology' is a rubric for dealing with ideas about language structure and use relative to social contexts" (110).

10 The UNESCO conventions dealing with tangible or intangible cultural property are, in reverse chronological order, Protection and Promotion of the Diversity of Cultural Expressions (2005), Safeguarding of the Intangible Cultural Heritage (2003), Protection of the Underwater Cultural Heritage (2001), Protection of the World Cultural and Natural Heritage (1972), Fighting Against the Illicit Trafficking of Cultural Property (1970), Protection of Cultural Property in the Event of Armed Conflict (1954), Protection of Copyright and Neighbouring Rights (1952). 
tions have the result that various actors are brought together in the context of cultural property debates, so the TK, TCE and GR issues debated reflect the interests of member states, civil society organizations, as well as indigenous or local communities. The communicative practices and perspectives on substantive issues of the committee are for this reason not only of importance to WIPO and the intellectual property system, but also to various other fora and contexts on the national, regional and international level.

How actors negotiate about cultural property in sessions of WIPO's Intergovernmental Committee on Intellectual Property and Genetic Resources, Traditional Knowledge and Traditional Cultural Expressions (IGC) allows for inferences about the processes and strategies in other negotiations as well as. It permits insights into broader perspectives on cultural property. Thus, this study goes beyond the scope of WIPO's IGC. By combining the analysis of communicative interactions with ethnography and including perspectives on macro-processes, it is able to contribute to the anthropological study of international negotiations, especially in, but not limited to the context of cultural property. Valuable insights can be gained by analyzing the pragmatics of negotiations at WIPO; they are of relevance for other bodies dealing with issues of TK, TCEs and GR as well, and moreover shed light on international negotiations from the perspective of linguistic anthropology.

\section{The Debate on Cultural Property}

The notion of cultural property is an "umbrella term" for the substantive issues discussed in WIPO's IGC. WIPO uses the terms TK, TCEs (also referred to as "expressions of folklore", EOF) and GR, and in addition, IGC participants make use of terms like "indigenous knowledge", "cultural heritage" and "cultural resources". As Regina Bendix has shown, using the example of "heritage", terms in this context have numerous contested denotations, veiled implications and ideological histories that have to be considered in analyzing discursive practices (Bendix 2009a, 2009b; see also Groth 2010a, Groth 2010b).

The ambiguity in terms contributes to their specific manifestations in the debates. The term cultural property as a meta-term incorporates differing uses, perceptions and perspectives on processes in which tangible or intangible culture artefacts are negotiated, whether in terms of social, political, economic or legal aspects. Therefore, the lack of specificity of this term allows for an inclusive view that understands the phenomenon as broadly manifested and does not exclude aspects of this debate from the outset. Cultural property as a term thus refers to UNESCO processes as well as CBD negotiations, to material cultural artefacts as well as discourses on repatriation and restitution, and spans a continuum from an international process like WIPO's IGC to local discussions of cultural heritage. The term will be more narrowly specified when contextually necessary. 
Anthropology has been preoccupied with the commodification of culture and cultural expressions, and not just since Michael Brown's programmatic monograph "Who Owns Native Culture?" (2003). There has been an ongoing, extended discussion of the intricacies involved in bringing culture to the market, preventing the misappropriation of cultural expressions and knowledge and protecting and preserving world heritage, whether in the "discovery" of specific cultural practices as ideological and monetary assets, UNESCO's conventions on safeguarding cultural heritage, or WIPO's Intergovernmental Committee on Intellectual Property and Traditional Knowledge and Folklore.

Economic and political processes draw attention to aesthetically flagged cultural artefacts and practices have been the subject of anthropological and folkloristic research since the 1960s. Positions taken with respect to "folklorism" and "fakelore" in the 1990s, which were initially very polarized, led to the realization that the canonization of culture by the cultural sciences was itself being reflected back into society. Thus, such canonization contributed to the ideological and monetary valorization of specifically marked culture (Tauschek 2012: 57ff, Bausinger 1991). Cultural anthropology and ethnology have since been engaged in debates about the reification and commodification of culture and cultural goods (Appadurai 1986, Barber and Lem 2004, Binsbergen and Geschiere 2005), though systematic examinations of historical and social genealogies of cultural property debates and related values are missing.

The study of the relation between tourism and culture also has been scrutinized, opening a door to the analysis of a "fourth world" which is involved in the commercial and ideological exchange of goods (Graburn 1976). It called for an ethnographical approach to layers that lie between and beneath cultural surfaces, in order to better understand the practices and strategies when culture is used in situations of contact and conflict. Research on tourism led to a theoretical approach with regard to the "museification" of culture and to UNESCO's efforts with respect to cultural heritage. Barbara Kirshenblatt-Gimblett's work (1993, 1998) on this topic is path-breaking, as it does not focus on heritage goods but on the processes by which they emerge. Kirshenblatt-Gimblett coined the term "metacultural operations" for these phenomena, which allows for an investigation into the various temporal, spatial and cultural spheres and processes of valorization that are involved. ${ }^{11}$ This focus results in conjoining heritage practices that intend to establish identity to questions of property rights.

Even so, to date there are far more case studies about heritage than about the predicaments of cultural property. However, there are exceptions, starting with Kasten's edited volume (2004) on Siberian examples of concepts of property and of the commons that include questions both on the construction of identity and

11 The notion of "metacultural operations" has been criticized for creating a dichotomy between “culture pre and post UNESCO proclamations" (Tauschek 2011: 60). 
about economic utilization. The connection between cultural property questions and an anthropological account of cultural heritage (Graburn 2001, Bendix 2007, 2009a) sheds light on political, economic and identity aspects of cultural property that are frequently discussed at WIPO and related fora (Hafstein 2009, Skrydstrup 2012). Dorothy Noyes has drawn attention to the influences cultural property processes have on social dynamics and structures (Noyes 2003, 2007; see also Tauschek 2010) and on the different conceptualizations of creativity and innovation that lead to cultural property (Noyes 2006; see also Hafstein 2004, 2008, 2009). A special issue of the journal "Ethnologia Europae" edited by Regina Bendix and Valdimar Hafstein (2009) highlights the "triad of culture, politics and economics" (10) which serves to constitute the terms of reference for ethnological debates on cultural property. An interdisciplinary edited volume by Bendix, Bizer, and Groth (2010) sheds light on the processes of constituting cultural property from economic, legal and anthropological perspectives. The "institutionalization" of cultural heritage and cultural property studies is illustrated both by two chapters in a recent "Companion to Folklore" (Hafstein 2012, Skrydstrup 2012) and a paper on the "principal points of irritation" in debates on traditional culture (Noyes 2010: 1).

Thus, the analyses of debates about cultural property are well documented, and they include many aspects relevant to IGC negotiations. However, even if some of the studies noted above focus on international processes (such as those in WIPO's IGC or the various UNESCO bodies), they typically do not analyze the specific negotiation practices. An understanding of how these negotiations work, how actors bring their positions and perspectives to the negotiating table, and how different interests and motivations are mediated in order to produce results that are either more or less substantial, is of central importance to for understanding the global processes involved in addressing cultural property. This study contributes to these questions both at the level of negotiation practices and at the level of substantive discussions of cultural property.

\section{Approach and Aims of this Study}

This study draws from fieldwork and participant observation of the sessions of WIPO's IGC on Intellectual Property and Genetic Resources, Traditional Knowledge and Traditional Cultural Expressions in Geneva in February 2008, October 2008, June 2009 and December 2010. It also draws on fieldwork conducted at WIPO's Conference on Intellectual Property and Public Policy Issues in Geneva in June, 2009. More formal interviews were carried out with WIPO staff members, delegates from WIPO member states, representatives of non-governmental organizations (NGOs), indigenous and local communities (ILCs) and other IGC participants, in addition to various informal conversations before, during and after the 
meetings. Field notes as well as audio transcriptions were used in the analysis, complemented by an extensive document analysis, including IGC working documents and reports, WIPO publications, and related material.

Using the methodological and theoretical frameworks of linguistic anthropology, including the ethnography of communication and linguistic pragmatics, the study aims to analyze communicative patterns and strategies in international negotiations. It takes into account structural as well as dynamic aspects of these negotiations, trying to shed light on the relation between them. Pragmatic strategies used by committee participants to promote their interests are also scrutinized. Given the lack of tangible outcomes, this study asks how significantly divergent views on core issues can co-exist over a long period of time without direct confrontations of oppositional parties or a failure of the committee. The study of substantial discussions about cultural property that influence negotiation processes will complement this analysis.

The second chapter of this study provides the theoretical and methodological framework. It is derived from linguistic anthropology, with a specific focus on the ethnography of communication, and from pragmatics and metapragmatics. The basic premises of these fields provide the foundation for the analysis of communicative events in WIPO's IGC.

Chapter 3 - "Negotiating Tradition on the Global Stage" - describes the genesis and contexts of IGC negotiations. It introduces the various actors and their interests, as well as the various communicative events in an IGC session. The contextualization of communicative events in Chapter 3 helps in building hypotheses where strategies are not verbalized in interviews or participant observation. The position of actors can be deduced from the larger context, so that for example the utterance of a diplomat in a communicative event needs to be linked to the position of his or her country's national position. The transparency of meeting documentation allows for the tracing of such positions over a long period of time (2000-2011), and secondary literature on the IGC process complements the data.

In Chapter 4 - "The Pragmatics of IGC Negotiations" - I analyze the communicative strategies and patterns observable in the IGC. Drawing both on the theoretical framework outlined in Chapter 2 and the ethnographic contextualization of Chapter 3, this chapter then will examine the most substantial communicative patterns observed during fieldwork at the IGC. Initially, this chapter will include a discussion of the question whether the IGC can be conceptualized as a speech community in the sense of the term used in linguistic anthropology. Thereafter, a specific communicative event of IGC meetings, the so-called "opening statements", will be analyzed with a focus on their different formal and substantial aspects and in context of the broader meeting. Using the notion of different referential frames, the negotiations will subsequently be studied as a dynamic process 
that mediates between different perspectives and interests via pragmatic strategies. The practice of "micro editing" will be analyzed as a specific case of this process.

The main task of Chapter 5 - "Multiperspectivity \& Differentiation" - is to elucidate the diverging perspectives on TK and TCEs held by actors involved in cultural property debates, coupled with terminology and communicative events of the IGC. It will examine the multiple perspectives on the substantive issues that underlies the practices analyzed in Chapter 4.

The conclusion summarizes and suggests further research that is planned. It asks what impact the elicited communicative patterns have for participants in terms of communicative and strategic competence, and how they can affect their position in negotiations. It examines what can be derived from the theoretical framework of linguistic anthropology and pragmatics for the study of international negotiations on cultural property. 


\section{On Terminology: Why Language Matters}

Language is not a neutral medium that passes freely and easily into the private property of the speaker's intentions; it is populated - overpopulated - with the intentions of others. Expropriating it, forcing it to submit to one's own intentions and accents, is a difficult and complicated process.

Bakhtin 1983: 294

The notion of terms as something stable, fixed, technical or neutral, or in other words, as things whose significance in a given context may be simply declared, has been criticized extensively, and for good reason. ${ }^{12}$ Throughout the course of this book, however, this notion will be revisited numerous times. Terms, and words, exist in contextual relationships that determine their meaning, their connotations and denotations. Context is not limited to the composition of words in sentences and texts. It also includes social and cultural factors, such as the status of a speaker or author, and the setting in which the term is used. Consider the term "tradition": it can point to valued practices developed over time and esteemed by a group of social actors. It can also be used in a negative manner or in contrast to "modern" and "better" practices. Then, "tradition" can have legal implications. When it is used in legal texts, what qualifies something as "traditional" must be defined, and such a definition differs from the two previous uses of the term. The perspectives on the term and on what it signifies are manifold. They range from appreciation to

\footnotetext{
12 For broad and basic critiques, see Adorno's lectures on Philosophische Terminologie (Adorno 1997); Wittgenstein's Tractatus Logico-philosophicus (Wittgenstein 1963), Bakhtin's work on heteroglossia (Bakhtin 2007), Fabian's "Taxonomy and Ideology" (Fabian 1977), or - for a more recent view from linguistic anthropology - Silverstein 2004.
} 
critique, from its emotive to its definitional qualities. Taken out of context, it is difficult to infer the meaning of such a term only with reference to its definition in a dictionary. Neither is the syntactical relationship of such a term to other parts of an utterance sufficient for fully understanding its meaning. A broad range of factors, external to the immediate occurrence of a term, influences its meaning. This observation has been the starting point for a number of fields interested in understanding and analyzing the relationship between language and language use, and thus the relationship between linguistic systems and their social and cultural contexts. These fields include linguistic anthropology, linguistic pragmatics, and the ethnography of communication. Their specific orientations, theoretical and methodological starting-points, and relevance to the subject of this book will be discussed in greater detail in the course of this chapter.

The premise they all share, namely that language has to be viewed in its social and cultural context, leads to the observation that the (potential and actual) use and implications of terms need to be scrutinized. Tradition is one such term that, within scholarship and especially in the disciplines of folkloristics and anthropology, has been criticized extensively..$^{13}$ Mainly the notions of tradition as something organic and authentic are viewed as problematic. Instead, scholars have pointed out the intentional use and construction of tradition for differing purposes. Tradition is thus not only a concept, but it can be a means to accomplish objectives and pursue interests. Do actors in international negotiations talking about traditional knowledge evoke the same critical concept of tradition as scholars? How can one distinguish everyday usage of the term from the heavily criticized and reflectedupon concept as used in folklore studies? Does the scholarly effort to clarify terms have an influence on how taxonomies are created and used within a group of social actors? More generally, how should one treat the everyday use of terms relative to the critical use of terms in scholarship? These questions are especially important when the object of study is embedded in everyday language use (Adorno 1997: 29): if the uses of tradition are intentional and strategic, the uses of the term are contingent on these intentions and strategies as well. Hence, the different uses of the term entail perspectives on what it signifies, and these perspectives are coupled with interests. Similar to the insight from anthropology and folkloristics that tradition is used intentionally, employing the term "tradition" is strategic as well. This is true for other terms, too: they are based on specific perspectives on their subject matter, and they are often used strategically. The analysis of terms must take this variability and bias into account.

13 See Glassie 1995, Dorson 1976, Ben-Amos 1984, Handler and Linnekin 1984, Bausinger 1969, Noyes 2009 or Bendix 1995: 189 for discussions in folkloristics and European Ethnology. For discussions from the viewpoint of the Frankfurt School, see Adorno 1986 and Benjamin 2008. For sociological approaches, see Shils 1981 or Jacobs 2007, and for a historical-constructivist perspective, see Hobsbawm and Ranger 1992. 
One principle of talk is to come to an understanding of a matter, to share information and ideas about something:

Our talk can cause others to reach, with us, an intersubjective identification of things. [...] [P] eople can use language to construct collectively reached and collectively consequential knowledge, opinion, and belief about all manner of things. In principle descriptive language can be fashioned into a tool or instrument for constructing sharable knowledge in the very event of communication. (Silverstein 2003: 89)

Yet, there is another layer to talk aside from the construction of shared knowledge. While the identification of things is part of discourse, talk is also a way for people to frame a matter according to their intentions. When people talk about tradition and other terms, their goal is not only to share knowledge. In situations where actors hold divergent views on a matter, contestation is as central to talk as understanding. Language is used to pursue one's interests and accomplish objectives. The descriptive quality of language is at times superceded by its pragmatic quality, and this is especially the case in international negotiations. It is important for a study on such negotiations to consider that terms are ambiguous, based on diverging perspectives, and used strategically.

By mapping the various uses of key terms, one can arrive at a more contextualized analysis, which is helpful in analyzing contingent terminology. One can in this manner establish how and in what (changing) contexts specific terms are used, and analyze the implications of the contextualized usages. Terminology might be interpreted in multiple ways or only one way, and terminology itself might be harnessed strategically or be used intentionally in specific interest constellations. In legal linguistics, this has been discussed in terms of the "vagueness" or "ambiguity" of terminology (Bhatia et al. 2005, Hutton 2009), with a focus on how to pragmatically deal with such uncertainties in legal interpretation (Münch 2002). Transcultural (Bhatia et al. 2008) and multilingual (Hilf 1973, Jansen 1999, Luttermann 1999, Triebel and Balthasar 2004) settings have been taken into account as well, but the emphasis is on questions of legal certainty and legal interpretation. However, the strategic and ideological ${ }^{14}$ implications of contingent (and thus ambiguous) terminology in differing sociocultural and political contexts have largely been neglected. Noteworthy exceptions, such as Alan Audi's "A Semiotics of Cultural Property Argument" (2007) do not limit themselves to formal analysis but provide a re-contextualization of terms in linguistic and ideological systems, allowing for a

\footnotetext{
${ }^{14}$ By ideological I refer to "belief systems" that are "fundamental or axiomatic", "stable" and "socially shared by the members of a collectivity of social actors" (van Dijk 2006: 116). They are connected to perspectives on terminology without necessarily being strategically harnessed. Strategies, on the other hand, are directly or indirectly based on belief systems.
} 
location and interpretation of terminology as it relates to its social and cultural embeddedness.

This chapter introduces the key analytical concepts employed in this study, setting the framework for the analysis of an intergovernmental committee that addresses questions of intellectual property (IP) and genetic resources (GR), traditional knowledge (TK) and traditional cultural expressions (TCEs). By looking at scholarly concepts and theories from a variety of fields whose interest is in language and society, the ethnography of communication, and linguistic pragmatics, one can construct a theoretical and methodological framework for analyzing the communicative events that take place during international deliberations on cultural property. Chapters 4 and 5 address questions concerning the function and ambivalence of terms used in everyday life as contrasted with the role language plays in negotiations about TK and TCEs.

\section{Linguistic Anthropology}

Language is a universal and essential capacity of mankind; study of language sheds light on buman nature as a whole, and study of languages is indispensable to an understanding of the concrete historical forms taken by buman nature.

Hymes 1975: 348

A starting point for the analysis of communicative events is the basic observation that people more often than not use language as a tool to communicate to each other how they see the world and how they want it to be. The prerequisite for this communicative processes is a shared language or a shared understanding of language. While anthropology is - according to a very broad and generalizing definition in numerous works and studies - the study of humankind, linguistic anthropology concerns itself with the role language plays in culture. The anthropological sub-field of linguistic anthropology emerged from the Boasian paradigm of cultural relativism. As cultures came increasingly to be seen from the view of their members, and not as developmental stages on an evolutionary ladder, the focus also shifted away from normative judgments to holistic and emic descriptions of social life and cultural practices. Part of this new paradigm was the attention paid to language and language use, including the relations between language and culture (see Boas's 1911 Handbook of American Indian Languages). From this emerged 
the study of language and culture or the view of "language as a cultural resource and speaking as a cultural practice" (Duranti 2002: 8899). ${ }^{15}$

Central to linguistic anthropology is thus the study of language in context, and a focus on the speakers of a given language or the participants in a speech community, and their communicative interactions. The focus of study came to be on the performative aspects of language use rather than on the competence to speak a language. ${ }^{16}$ This distinction draws on de Saussure's notions of langue, the underlying linguistic structures and the arbitrary relation between signs in a semiotic system, and parole, the actual communicative events where speakers draw from langue to form utterances (Duranti 1997: 14). Duranti outlines three central theoretical concepts for linguistic anthropology that have been of lasting importance: performance, indexicality, and participation.

The first - performance - is an interest in how language is actually used, and thus the 'things people do with words' (Austin 1962). Not the grammatical structures of language and the rules for use and interpretation - what Chomsky terms "competence" -, but mainly the way people interact by use of language are central to the study of language in linguistic anthropology: "Competence in this case is the knowledge of a language that an ideal speaker has. Performance instead is the implementation of that knowledge in acts of speaking" (Duranti 1997: 14). In contrast to Austin's use of the term, performance in linguistic anthropology is not limited to describe what can be achieved by the use of language. It includes the additional dimension of how language is used, and by what performative principles such as aesthetic criteria or the appropriateness of an utterance - listeners evaluate what is being said (15). Then, there is an interest in linguistic anthropology in the creative aspects of performance, that is the ways in which speakers change, manipulate, or reproduce these evaluative principles (16).

The concept of indexicality concerns the social functions of language and the intentions speakers have when using it. For example, language can be used to point to objects, to signal the social status of its speaker or listener, or a speaker's choice of words can hint at his political stance or intentions (18).

Participation - the last concept - is an interest in those who make, use, receive and interpret linguistic utterances (20). This concept points to the fact that taking part in communicative events also means being part of a social group with implica-

\footnotetext{
15 Much attention has been paid to definitions of the field, with further differentiations made between the terms "linguistic anthropology", "anthropological linguistics", "ethnolinguistics" or sociolinguistics" (Duranti 2001, Salzmann 1993) and specific attention to the historical development of the discipline in different contexts (Duranti 2003, Gumperz and Cook-Gumperz 2008, Rampton 2007). Here it suffices to briefly outline the basic assumptions of the field.

${ }^{16}$ Chomsky used this to differentiate between competence and performance (1965), though this was criticized by Dell Hymes, among others, who argued for a focus on performance and that the two could not be separated.
} 
tions for the use of language. In short, the connectedness of participants underlines the social and political contexts of language use. In contrast to atomistic communicative models of 'speaker' and 'hearer', the concept of participation pays attention to social dynamics and other contextual variables influencing what is said, how and with which intentions it is said, and which evaluative principles apply to a communicative event.

These three aspects of performace, indexicality and participation are of central importance for this study, and are also part of the ethnography of communication.

\section{The Ethnography of Communication}

For the anthropological study of behavior there is another area of importance, one that seems general, central, and neglected. It can be called the ethnography of speaking.

Hymes 1962: 13

The ethnography of speaking and communication emerged in the late 1960s in the United States out of common interests in linguistic anthropology, sociolinguistics and folklore. It now has a permanent place in the cultural sciences there (Duranti 1999a, 2004, Keating 2002, Saville-Troike 2003). In developing a theory of language use by systematic ethnographic methods, Dell Hymes (1964) saw a means for analyzing the role of language in culture and society in a manner that went beyond the scope of a more theoretical linguistics.

Central to this is the speech community as the basic unit of analysis. It denotes a group sharing specific rules with regard to language use, and a shared understanding of communicative interaction and interpretation (Hymes 1986: 53-55). Beyond an interest in grammatical and vocabulary-centered topics, the ethnography of communication extended the analysis of "how to do things with words" (Austin 1962) to argue that the complex socio-cultural norms of speech communities, together with their speech genres, allow for inferences about other components that constitute culture (Hymes 1979). By recording and closely analyzing what are ultimately immaterial speech events and performances, poetic as well as political dimensions of culture can be identified, and profane insights, spiritual principles and ideological aspects be shown (for examples, see Gumperz and Hymes 1954, Bauman and Sherzer 1989, and Gumperz and Cook-Gumperz 2008).

Such studies go beyond the original focus on indigenous populations in the Americas, and extend the focus on verbal performance to written material and new media. Richard Bauman (1983) has applied the approach to the analysis of historical source material to illustrate the ideological principles of Quaker practices with respect to speech and silence. Charles Briggs (1996) used the approach to draw 
attention to gender and age-related hierarchies and the contestation between Mexicans and other ethnicities in New Mexico (1996). Briggs also worked out a research manual, entitled "Learning How to Ask" (1986), that focuses on communicative competence and the need to be aware of communicative aspects while conducting fieldwork. He has extended this in analyses of the political and cultural effectiveness of language ideologies (Briggs and Mantini-Briggs 2003, Briggs 2005, 2007, Bauman and Briggs 2003). Susan Gal uses the approach to examine the relations between multiple language regimes and ideologies in the political arena ( $\mathrm{Gal}$ and Woolard 2001, Irvine and Gal 2000, Gal 1998, 2008b). Bodies of knowledge are conveyed and transformed in communicating, with the specific mode signaling how the message is to be received. In the case of non-communicating, messages may be kept secret or forgotten. The ethnography of speaking has also been used for analyses of the role of speech in political events (Brenneis and Myers 1984, Gal 2008a) and conflicts (Brenneis 1988), as well as in the study of political representation and communicative performance (Yankah 1995, Gal 2008b).

Central to the ethnography of speaking is the SPEAKING-model (Hymes 1964). It consists of eight factors and sixteen components that provide a guide for ethnographically analyzing communicative events. These are briefly outlined here and taken up in the subsequent chapters, using WIPO's IGC as an example.

The first factor is situation, its components the setting and the scene of linguistic interactions. While the setting denotes the material characteristics of a speech event, the scene signifies the "'psychological setting' or the cultural definition of an occasion as a certain type of scene" (Hymes 1986a:60). On the one hand, the scene refers to aspects such as locality, architecture, the design of rooms or the availability of microphones and speakers. On the other hand, the term also takes into account participants' understanding of how the setting is defined in relation to other speech events. Within a given setting, the interaction may be redefined "as a changed type of scene, say, from formal to informal, serious to festive, or the like" (60).

The second factor is participants, whose components are speaker, addressor, hearer and addressee. The distinction derives from the insight that the speaker of an utterance is not necessarily the addressor in cases where actors speak on behalf of another entity. Similarly, the hearer or recipient of an utterance does not need to be the addressee in situations where multiple audiences are addressed (61).

The third factor - ends - consists of the outcomes and goals of speech acts. Here, "the conventionally expected or ascribed must be distinguished from the purely situational or personal, and from the latent and unintended" (61-62). What is taken into consideration are both the intentions and motivations of participants, as well as the unintended or unforeseeable consequences of a speech event.

The fourth factor Hymes describes is the act sequence, consisting of message form and message content. While the first of these two components denotes how 
something is said, the second relates to what is said. Hymes gives the example: "He prayed, saying '..." which refers both to form and content, in contrast to "He prayed that he would get well”, which only reports message content (60).

The fifth factor, the key, denotes the "tone, manner, or spirit in which an act is done" (62) and finds its expression in verbal or non-verbal cues.

Hymes calls the sixth factor instrumentalities, and argues it consists of the components channels - as in the different modes of communication, such as speaking, writing or singing - and forms of speech - meaning dialects, language varieties, registers and code (63).

The seventh consists of norms, both of interaction and of interpretation. These point, in turn, to aspects of speech that regulate turn-taking or forms of address and to the contextual delineation of how an utterance can be interpreted by the various recipients (64).

Finally, the eighth factor, the genre of a speech event, denotes "categories such as poem, myth, tale, proverb, riddle, curse, prayer, oration, lecture, commercial, form letter, editorial, etc." (65). Hymes thus includes the formal characteristics of speech, making it possible to recognize conventional forms of speech and to use and interpret them accordingly.

The ethnography of communication including these components is qualified for the research on the IGC which constitutes a dynamic communicative arena that remains fragile as to functional intercultural communication. At the same time, the IGC is a locally situated example for communicative patterns of experience in globally connected spaces. A significant part of the WIPO committee is the communication among members who do not share the same origins, language, material culture, or world-views. This communication utilizes face-to-face meetings as well as virtual and print communication to create a communicative community that means to include, if not necessarily represent, "the world" and hence to create 'global' guidelines for cultural property. 


\section{Pragmatics \& Metapragmatics}

Pragmatics will have as its domain speakers' communicative intentions, the uses of language that require such intentions, and the strategies that hearers employ to determine what these intentions and acts are, so that they can understand what the speaker intends to communicate.

Davis 1991: 11

Metapragmatics, as it has grown out of the Jakobsonian paradigm, beginning ostensibly as a means of understanding the linguistic signal, has evolved from this purely linguistic modeling to include broader semiotic activity, now a means by which to conceptualize and explain the appropriate functioning of these signs in pragmatic usage.

Pressman 1994: 482

Pragmatics is the branch of linguistic anthropology concerned with language use and its social and cultural functions. It can be described as the study of the meanings of linguistic signs relative to their communicative and social functions. However, it has been difficult to define the field of pragmatics in a precise way that does not leave anything out and that at the same time is not too broad. ${ }^{17}$ Probably the most important aspect in this field of study is the programmatic distinction in semiotics between syntactics, semantics and pragmatics. ${ }^{18}$ While syntactics studies the formal relationship between signs, semantics looks at the relationship between signs and the things they represent. Pragmatics examines the relationship between signs and the interpreters of signs. Semantics, the "study of meaning in linguistic signs" (Silverstein 1976: 190) is limited in its scope as it does not pay attention to language use and to the contexts of linguistic utterances. Levinson attempts to weigh the pros and cons of a number of definitions, one of which is that:

Pragmatics is the study of the relations between language and context that are basic to an account of language understanding. (Levinson 1983: 21)

\footnotetext{
17 “Levinson's extremely careful weighing of the pros and cons of various definitions does not lead much further than the vague notion of pragmatics as the study of meaning in context (given substance only by ostensibly defining it as the study of whatever phenomena Pragmatics discusses). Though such a definition may lead to the acceptability of the claim that semantics deals with truthconditional aspects of meaning whereas pragmatics is concerned with aspects of meaning that cannot be accounted for in terms of truth conditions, it certainly does not support a boundary between pragmatics and sociolinguistics." (Verschueren 1985: 460).

18 This distinction dates back to Morris's "Foundations of the Theory of Signs" (1938) or Carnap's "Foundations of Logic \& Mathematics" (1951).
} 
Central here is the notion of context, used as a means to separate linguistic semantics from linguistic pragmatics.

Indeed, this distinction - however problematic and inadequate may be - is crucial for grasping the scope of pragmatics. The subject of the field is the study of language use, involving not only the semantic message, one constructed according to grammatical and syntactical rules, but also all the other factors that Hymes lists in his SPEAKING-model: the situation, participants, ends, act sequence, key, instrumentalities, norms of interpretation and interaction and genre.

Hence, the scope of pragmatics extends to such aspects as the conditions for an understanding of language, not only with regard to semantics, but also in relation to the social and cultural context of language use. That may be characterized by factors such as the appropriateness or felicity of an utterance, the communicative competence to use and interpret utterances, or the exploitation of communicative forms for specific functions. It can also involve the use of honorifics to signal differences in social status or the existence of conversational implicature ${ }^{19}$ that communicates a second pragmatic message. More generally, pragmatics deals with the different functions speech can have (Jakobson 1960) in the various contexts of its use. Thus, it goes beyond the study of semantico-referential meaning, whose signs are "the aspect of meaning which describes events in the world that are independent of the circumstance they are uttered in" (Silverstein 1976: 14) as opposed to indexical meaning, the "meanings of linguistic signs relative to their communicative functions" (20) in a given context. Thus, both the ethnography of communication as outlined by Hymes as well as pragmatics are concerned with specific instances of language use, its functions and implications.

But are participants in communicative events aware of the communicative functions that are activated by utterances? Discussions of communicative competence (Hymes 1971) partly address this, as it relates to the requirements actors need to meet to communicate adequately.

Metapragmatics also addresses this question. Its study is of "referential event[s] in which pragmatic norms are the object of description" (Silverstein 1976: 48). As Charles Briggs puts it, "much can be gained by distinguishing the complexities of how signs circulate from the way that people represent signs and attempt to regulate their uses" (Briggs 2012: 97).

The distinction between social and discursive functions of utterances (pragmatics) and talk about these functions (metapragmatics) is especially vital to the analysis of discursive practices in zones of contestation. The intentional coding of events or strategically highlighting event features uses the multi-functionality and indeterminacy of utterances, as Charles Goodwin has shown in an exemplary

\footnotetext{
19 The notion of "conversational implicature" refers to "something which is implied in conversation, that is, something which is left implicit in actual language use" (Mey 1993: 99). See also Carston 2004.
} 
manner for courtroom argument (1994). Thus, by looking both at pragmatics as the social functions of utterances and at metapragmatics as the awareness of these functions and the competence to exploit them, the communicative strategies speakers use to reach their goals can be analyzed.

Context is of central importance here. On the one hand, the knowledge of context is essential for participants of a communicative event to choose how to mediate their perspective on a given subject (e.g. to convince a jury of the guilt or innocence of a defendant) or to choose a coding scheme for an utterance appropriate to audience and context. This knowledge of context is also vital in analyzing such speech events. To understand the perspectives taken or the coding schemes of participants in communicative events, it is both important to distinguish between semantics, pragmatics and metapragmatics (by way of linguistic analyses) and to focus on the context of an utterance. As Silverstein puts it, "the use of some particular word or expression at a moment in denotational text-time [...] comes differentially to invoke - to summon to the here-and-now - some specific cultural concept in a schema of such" (Silverstein 2004: 634). Thus, only analyzing text and talk would fail to account for contextual features that are linked to embedded cultural or ideological concepts. Pragmatics studies the meanings and functions of language use and utterances in relation to contexts, and metapragmatics allows for a reflection on the intentional or directed uses of such functions.

The approaches used in linguistic anthropology, the ethnography of communication, pragmatics and metapragmatics are very important for the analysis of pragmatic strategies, communicative patterns and the differential use of language, especially in, but not limited to, the context of WIPO. What one sees in this forum is a highly politicized use of words, and linguistic strategies or patterns that change over time, space and the context in which they are used. The context as well as the social and political functions of language play a central role in this committee, so the following chapters focus on them to gain an understanding of the communicative processes shaping negotiations on cultural property. 



\section{Negotiating Tradition on the Global Stage}

\section{February 2008}

The plenary room: busts of Aristotle on the left and Alexander the Great on the right. In front is the dais, with seats for the secretariat, the Director General and Vice Director General, and the chairpersons of the session. Behind it is an upholstered panel in ocher that separates the dais from the glass facade of WIPO's 13story headquarters in Geneva's UN quarter. The curtains are almost translucent. On both sides of the elevated dais stand tables with yellow nameplates of intergovernmental organizations: UNESCO, SBCD (Secretariat of the Convention on Biological Diversity), FAO (Food and Agriculture Organization), OMC (Organisation Mondiale du Commerce or World Trade Organization), the African Union, and others. Cameras on pivots can pan across the whole room, and are used to broadcast what happens in the main room to an adjacent, smaller room. ${ }^{20}$ Rows of tables, arranged in semi-circles and facing the dais, reach to the back of the room. On each of table stand adjustable microphones and earphones for simultaneous translation. Using two arrows, one can choose between no translation, English, French, Spanish, Russian, Arabic and Chinese. Also to be found on the tables are blue nameplates with the engraved names of WIPO member states, in French. Delegations are seated in alphabetical order, so Afrique du Sud sits at the front and to the right while Zimbabwe is at the back and to the left. The last several rows of tables and a row of chairs at the back are reserved for NGOs and for "indigenous and local communities" (ILCs). Permanent observers of the committee have their

\footnotetext{
20 This system is sometimes also used for live online broadcasts of WIPO sessions, such as of the General Assembly (GA) in 2010.
} 
"own" engraved nameplates in red, others are provided with makeshift acrylic glass holders and black-and-white printouts of their delegations' acronym. The glasspaned booths for interpreters at the back oversee the plenary room (Fig. 5).

It is the first day of the 12th session of WIPO's Intergovernmental Committee on Intellectual Property and Genetic Resources, Traditional Knowledge and Traditional Cultural Expressions/Expression of Folklore (IGC on GRTKF). The meeting is scheduled to begin at 10 a.m. At 9:15 a.m., the plenary room and the foyer are almost empty, apart from WIPO staff and security personnel. Little by little, delegates arrive in WIPO's main foyer, filling out paperwork to register for the session and receiving their name badges, whose colors are similar to the larger name plates in the plenary room: delegates from member states are marked blue, observers green. During the meetings, these are an important way participants can establish the identity and status of their counterparts, as well as determine whether one is granted access to internal meetings or confidential information. Even in the cafeteria, they aid IGC participants in learning whom to speak to when and about what. Security guards are posted in front of each of the doors of the plenary room. They check the name badges and prohibit entrance to participants not authorized to attend specific meetings. At the left door stands a photocopier, next to it several tables stacked with brochures, announcements and the various documents relevant for the subsequent meeting. Behind a counter, WIPO staff members hand out additional documents from prior sessions and provide new documents that are produced during the course of the session. A few steps away from the main plenary room is Room B, partly used by observers and the staff assistants of member states who cannot find a seat in the main room. It is equipped with microphones, earphones and booths for simultaneous translation, and is also used for side-events, regional group coordinating or introductory meetings.

At 10:10 a.m., the main room is still not full, and delegates walk around or stand chatting in small groups. A small display in the foyer indicates an internal meeting of the African Group on the thirteenth floor. Finally, at around 10:30 a.m., the bulk of the delegates have arrived and WIPO's vice Director-General signals with his gavel that the session is about to begin.

The session starts with the election of a chair and vice-chairs for the next several meetings of the committee. After a proposal by Romania and approval by Russia, the elected chair takes his seat on the dais and after a few words of introduction suspends the session until the early afternoon. What follows is the so called "indigenous panel". In 2008 this was a relatively new segment at the beginning of each IGC session where representatives of ILCs could present their views on the issues discussed in the committee. During the lunch break, delegates and participants either gather for side-events, internal consultations or head to the 


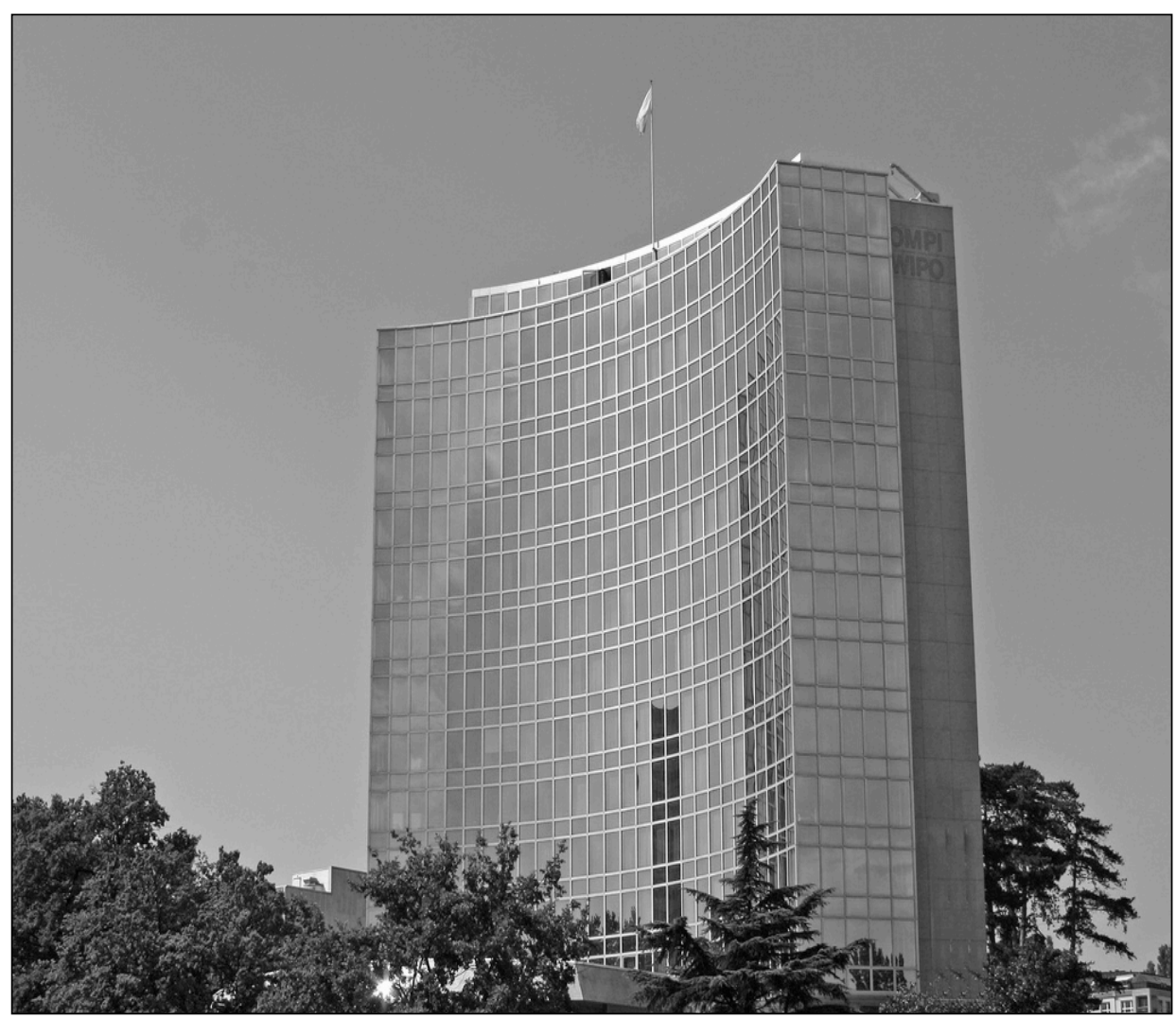

Figure 1. WIPO's main building in Geneva. Photo by Ville Oksanen.

WIPO cafeteria, situated in a connected building accessible by an airy underground hallway. ${ }^{21}$

In the cafeteria, delegates reconnect, talk strategy or just chat in small groups. In terms of fieldwork, the cafeteria and other hallway events are an important venue, since one sees who talks with whom, or one can approach delegates informally and talk about their perspective on the upcoming meeting. The more formal plenary session recommences at about 3:30 p.m. The election of the chairs has to be repeated due to an inconsistency in the agenda, and again, Romania makes the proposal, Russia approves it, and all other delegations silently assent. After adopting the agenda and the report of IGC 11, and comments by Algeria and Mexico on the wording of the report that are not replied to, additional organizations are accredited for participation in IGC meetings as observers. For the rest of this first

${ }^{21}$ In another adjacent building is a second cafeteria primarily used by WIPO staff. Since 2011, there is a third cafeteria in a new WIPO administration building next to the main building. 


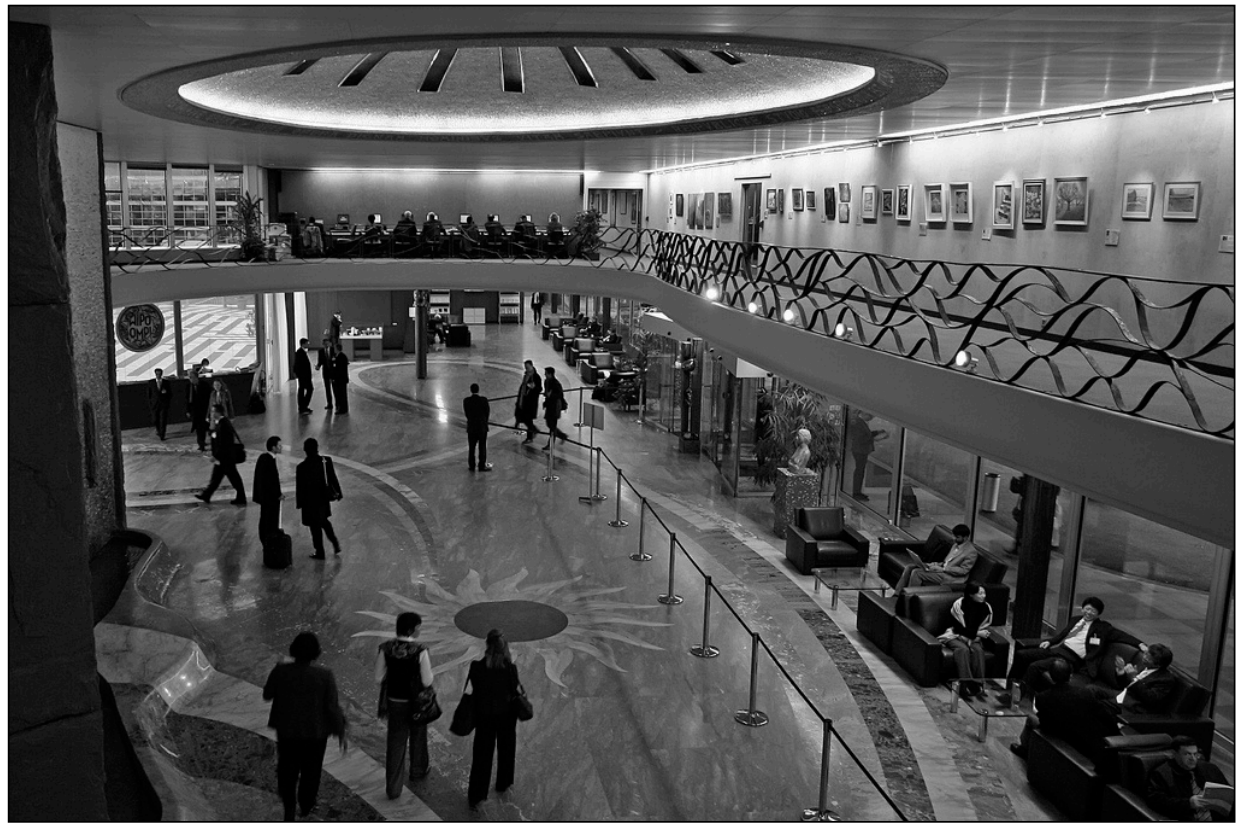

Figure 2. Foyer of WIPO's main building in Geneva. Photo by Ville Oksanen.

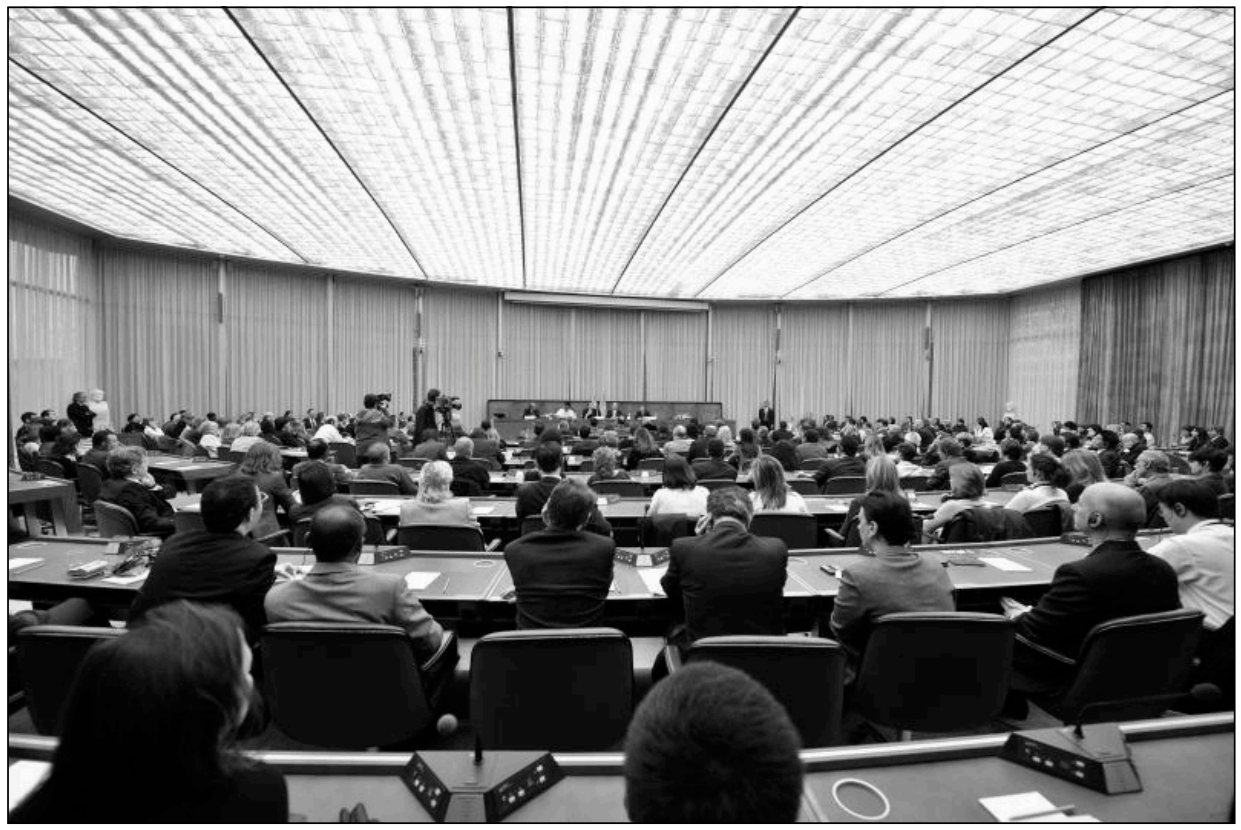

Figure 3. WIPO's main plenary room in Geneva. Photo by UN Photo/Jean-Marc Ferre. 


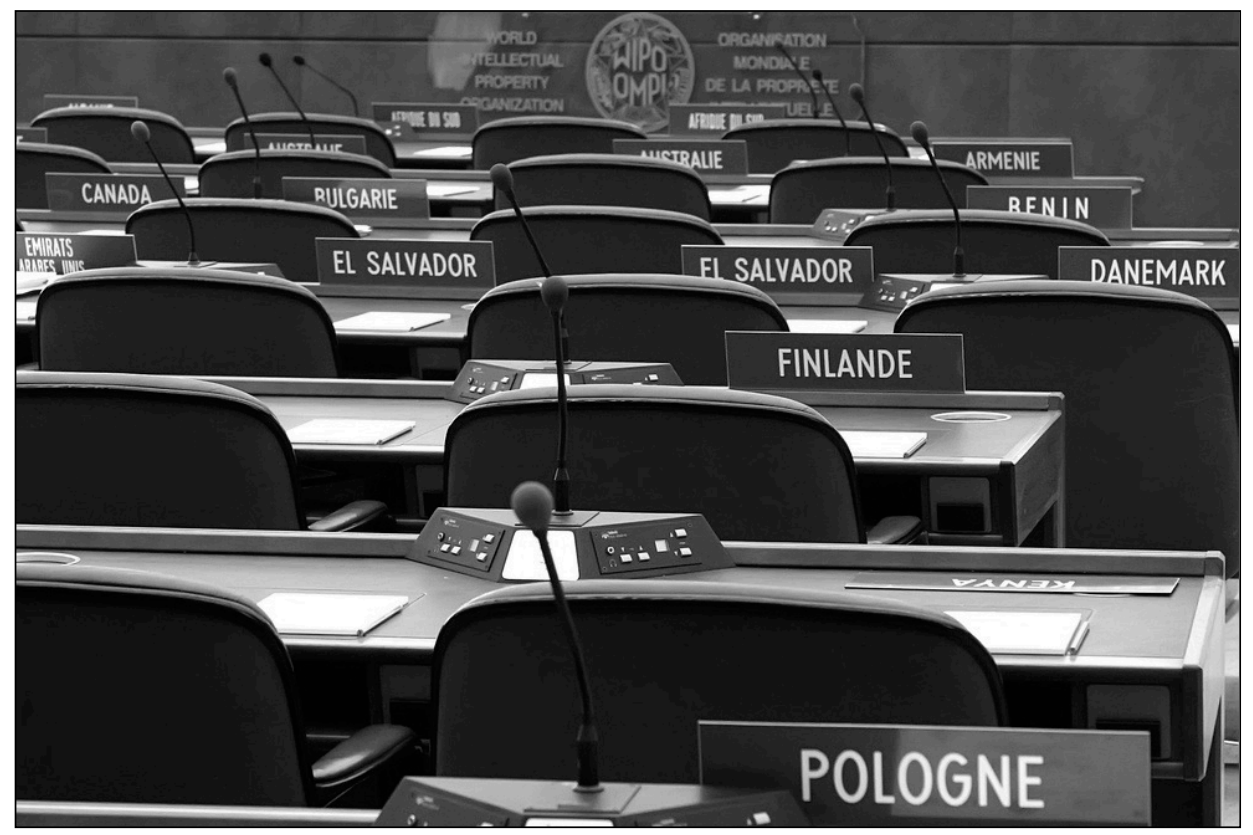

Figure 4. Seats of national delegations in WIPO's plenary room in Geneva. Photo by Ville Oksanen.

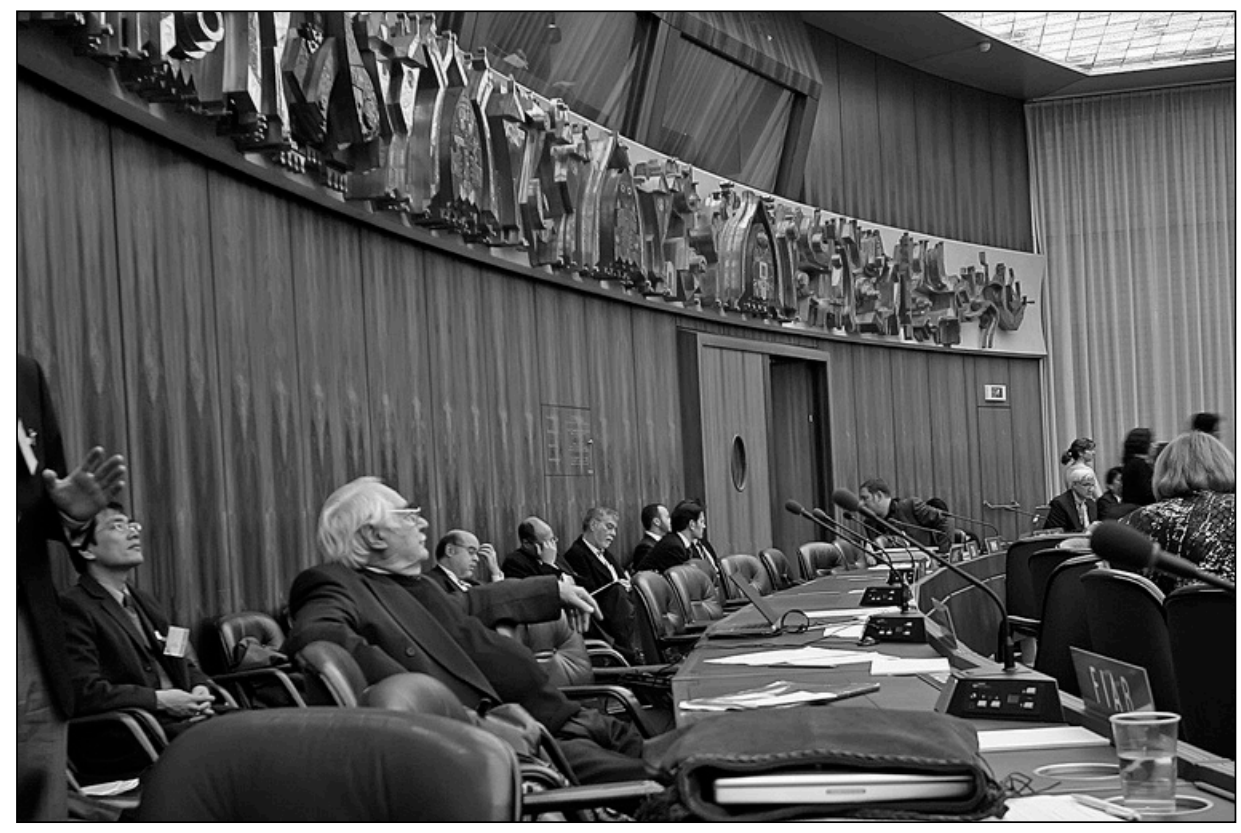

Figure 5. Back rows of WIPO's main plenary room in Geneva. Photo by Ville Oksanen. 
day, delegation after delegation delivers their opening statements, including a thank you to the chair, the expression of appreciation for the documents and the support provided by the secretariat, and express a positive outlook on what is to follow in the next days. ${ }^{22}$ As it draws nearer to 6 p.m., the session's chair suspends the meeting, and regional group coordinators announce the time and venue for internal consultation the next morning. While WIPO meetings almost never start on time, they seldom end late: it is a concession to the Genevan union of interpreters. The day ends with a reception in WIPO's main foyer, where hors d'oeuvres, wine, whisky and soft drinks are served. The mood is relaxed and delegates talk informally.

For the following four days, delegates and observers discuss matters of TK, TCEs and GR in the main plenary as well as in smaller regional group meetings and informal settings. At the end of the week-long twelfth meeting of the IGC, the committee will have decided - besides smaller procedural items - to compile two gap analyses for the next session. They are to describe "what obligations, provisions and possibilities already exist at the international level to provide protection" for TK and TCEs and "what gaps exist at the international level, illustrating those gaps, to the extent possible, with specific examples" (WIPO/GRTKF/IC/ 12/Decisions).

After seven years of negotiations, this decision is a clear indication that progress is very slow and that there is a lot of dissent between member states on how to proceed with the committee. In part, this study deals with the question how the IGC continues to exist besides this clear lack of consensus and some member states' lack of interest to move forward. How, after much preparatory work, twelve meetings and inter-sessional negotiations, is it possible that the only substantive decision on future work is to map the existing international legislation on TK and TCEs and gaps therein? Especially given the fact that there are some member states calling for a legally-binding international instrument for the protection of TK, TCEs and GR, this is a puzzling situation. IGC 12 included wider-reaching efforts and proposals to move the work of the committee in the direction of treaty negotiation, but they were mostly turned down by states not interested in this. While the session ended with critique and in conflict, in the end a fragile consensus with minimal results on future work was found - and this is a typical outcome of IGC meetings. More substantive agreements are very rare, and slow speed as well as adversarial positions resulting in decisions with only tenuous implications are what characterizes IGC negotiations. And despite delegations threatening to walk away from the negotiation table and bring issues of TK, TCEs and GR to other fora - an unwanted process for delegations not interested in new intellectual property legislation for cultural property -, the situation of the committee lingers on

\footnotetext{
${ }^{22}$ See Chapter 4 for an analysis of opening statements.
} 
without any bigger ramifications. Somehow, delegates seem to be able to create stability in an essentially unstable situation.

The main argument of this study is that communicative strategies play a key role in mediating the different interests of stalling and speeding member states, and that the analysis of linguistic behavior helps to understand what keeps the committee together despite only minimal progress in its work. The specificities of diplomatic registers and communicative practices are essential for an understanding of how stability is constantly recreated despite divergent views and interests. Thus, this study will analyze the sociolinguistic practices and patterns used by participants in international negotiations. It will shed light on how actors in WIPO's IGC employ communicative strategies in order to both mediate between different positions as well as enforce their interests while making only small concessions to opposing parties. While its results are partly applicable to intergovernmental negotiations in general, this study will focus on IGC negotiations on cultural property within the sphere of activities of WIPO.

\section{WIPO's Committee on Traditional Knowledge}

WIPO is one of the UN's specialized agencies. ${ }^{23}$ It was established in 1967 by the "WIPO Convention" and as a successor to the "United International Bureaux for the Protection of Intellectual Property" (BIRPI) set up as early as 1893 to administer treaties for the protection of industrial property (Paris Convention for the Protection of Industrial Property, 1883) and literary and artistic works (Berne Convention for the Protection of Literary and Artistic Works, 1886). Both treaties still exist (with amendments) and are nowadays administered by WIPO. The organization's mission statement reads:

The objectives of the Organization are: (i) to promote the protection of intellectual property throughout the world through cooperation among States and, where appropriate, in collaboration with any other

\footnotetext{
${ }^{23}$ The UN has fifteen specialized agencies which cooperate through the Economic and Social Council (ECOSOC). Notable UN organizations dealing with matters of traditional knowledge/traditional cultural expressions and/or concerns of Indigenous Peoples include UNESCO, the ILO, the WTO, the World Health Organization (WHO), the World Meteorological Organization (WMO), and WIPO. The WTO, the United Nations Permanent Forum on Indigenous Issues (UNPFII) - "an advisory body to the Economic and Social Council, with a mandate to discuss indigenous issues related to economic and social development, culture, the environment, education, health and human rights" (UNPFII 2011) - and the United Nations Human Rights Council (UNHRC) are three other important organizational bodies for the subject of traditional knowledge. All these organizations and their interrelation in negotiations about traditional knowledge will be dealt with in more depth in the course of this study.
} 
international organization, (ii) to ensure administrative cooperation among the Unions. ${ }^{24}$

The organization addresses intellectual property issues at the international level, which includes trademark, copyright, and patent questions, broadcasting treaties or "domain dispute settlements", such as the question who the rightful holder of the domain www.schweiz.ch is, to give a prominent example. ${ }^{25}$ WIPO is part of a global intellectual property infrastructure including a diverse range of organizations, frameworks and issues (Dutfield and Suthersanan 2008). The financial interests involved in these issues are substantial, as patents, trademarks and copyrights to protect intellectual property are of considerable value to stakeholders, especially corporations.

In 2000 , the $25^{\text {th }}$ session of the WIPO's GA discussed whether to establish an intergovernmental committee to address issues of intellectual property, traditional knowledge and folklore. It "invited member states to consider the establishment of an Intergovernmental Committee on Intellectual Property and Genetic Resources, Traditional Knowledge and Folklore (IGC)" (Dutfield 2003: 15). The text adopted by the representatives from WIPO's 184 member states at the GA argued that "[w]ith the emergence of modern biotechnologies, genetic resources have assumed increasing economic, scientific and commercial value to a wide range of stakeholders" (WIPO/GA/26/6:para.1). Furthermore, traditional knowledge associated with genetic resources as well as "other tradition-based creations, such as expressions of folklore" were receiving an increased amount of attention within a "globalized information society", making it necessary to rethink intellectual property questions in these three areas. The decision by the GA to bring this new intergovernmental committee to life read in part:

The Intergovernmental Committee would constitute a forum in which discussions could proceed among Member States on the three primary themes which they identified during the consultations: intellectual property issues that arise in the context of (i) access to genetic resources and benefit sharing; (ii) protection of traditional knowl-

\footnotetext{
${ }^{24}$ See http://www.wipo.int/treaties/en/convention/trtdocs_wo029.html, last accessed 1 May 2011.

25 This, as well as the mission statement, include ideological presuppositions about the purpose of the protection of intellectual property as well as the basic notion of intellectual property as an individual right. It has been argued for good reason that the basis of the intellectual property system are Eurocentric notions of creativity and property (Hafstein 2004, Noyes 2006). Yet, WIPO argues that forms of intellectual property can be found in all cultures and that it thus is a universal characteristic of mankind.
} 
edge, whether or not associated with those resources; and (iii) the protection of expressions of folklore. ${ }^{26}$

The IGC convened for its first session from April 30, 2001 to May 3, 2001, in WIPO's headquarter in Geneva, Switzerland. The agenda ${ }^{27}$ allotted time for discussions on the rules of procedure ${ }^{28}$ and, more importantly, about access to genetic resources and benefit-sharing, and the protection of TK and expressions of folklore $(\mathrm{EOF})$. These are - with a change in terminology from "expressions of folklore" to "traditional cultural expressions" (TCEs) ${ }^{29}$ - still the three substantive items discussed in the IGC. The threefold distinction stems from the structure of the intellectual property system - including international treaties, conventions and other legal frameworks regarding intellectual property rights - which differentiates between the aspects of knowledge, performance and biological resources. ${ }^{30}$ The IGC is one of many committees addressing intellectual property issues under the umbrella of the UN, and not one of the more publicly visible ones. Other WIPO or WTO committees on patents or copyright are much more influential and gain considerably more political and economic interest. The issues of TK, TCEs and GR discussed in the IGC are - at least at the moment - only of marginal importance for the broader international intellectual property regime.

At the end of IGC 1, the first meeting held, member states decided to convene for a second time in 2001 to continue the discussions about TK, TCEs and GR. ${ }^{31}$ The IGC's genesis, however, is much earlier. As early as the 1970s, WIPO cooperated with UNESCO in drafting the "Model Provisions for National Laws on the Protection of Expressions of Folklore Against Illicit Exploitation and other Forms

\footnotetext{
${ }^{26} \mathrm{WIPO} / \mathrm{GA} / 26 / 6$ : para.14

${ }^{27}$ WIPO/GRTK/IC/1/1 Rev.

28 The decision on this item included the accreditation of ad-hoc observers to the IGC. In contrast to other WIPO fora where only international organizations with close relations to both intellectual property and the specific issues of the committee are allowed to participate, the IGC is more open to the inclusion of other NGOs and ILCs. There has, however, been a change in the terms of participation. While observers at early IGC sessions had little opportunity to voice their concerns, it has become customary in the IGC that such participants be granted wide-reaching rights to speak and to contribute to the negotiation process, unlike in other, comparable fora. Yet, in the end only member states have the right to make decisions.

29 The rationale behind this change in terminology is to avoid using "folklore" since it can connote things regarded as outdated and of little or no value. This is given as a reason both by WIPO participants when asked about the reason for the new wording, and can also be found in the documentation of the WIPO process.

${ }^{30}$ See WIPO/GRTKF/IC/5/12 and WIPO/GRTKF/IC/6/3 on this distinction; see also the list of member state comments on this issue noted in the footnotes in WIPO/GRTKF/IC/7/5:11.

31 The main interest of this study is to analyze negotiations on cultural property, and this chapter focuses on TK and TCEs. The topic of GR is mainly discussed in its relationship to TK and TCEs, and most discussions in the IGC focus on either TK or TCEs. The role of GRs, both in relation to TK and TCEs and in pragmatic strategies, is discussed in Chapter 4.
} 
of Prejudicial Action" (WIPO/UNESCO 1985). However, these initiatives did not produce any other substantial results:

The involvement of WIPO in TK goes back more than 20 years. In the 1970s and 1980s, WIPO, with UNESCO, held a series of meetings on folklore that culminated in the 1982 adoption of the Model Provisions for National Laws on the Protection of Expressions of Folklore Against Illicit Exploitation and Other Prejudicial Actions. In 1984, WIPO and UNESCO convened a meeting to explore the possibility of developing an international treaty on folklore based on the Model Provisions. However, participants at the meeting were unable to reach agreement and the proposal for a treaty was withdrawn. Consequently, WIPO dropped the issue of folklore from its programmes for several years. (Dutfield 2004: 132-133) ${ }^{32}$

WIPO again took up issues of folklore and cooperation with UNESCO in 1997 at the World Forum on Folklore in Phuket, Thailand. The participants agreed on establishing a forum for further discussions about folklore and the intellectual property system, arguing that the existing copyright, trademark and patent system offered inadequate protection for TK, TCEs and GR. In 1998, WIPO established the Global Intellectual Property Issues Division (GIPID), to address, among other things, folklore and the possible creation of an international committee dealing with its relation to the international intellectual property system. From the beginning, its expected outcome was constrained:

GIPID's mandate is limited. American support for the new mandate was secured in return for the concession that GIPID was not "on a norm setting track"; that is to say, that its work is not intended to feed into a process which would end with the creation of a treaty or recommendations. (Halewood 1999: 986-987)

However, in 1998 and 1999, GIPID, which was later renamed the Traditional Knowledge Division, carried out nine fact-finding missions to the South Pacific, Eastern and Southern Africa, South Asia, North America, Central America, West Africa, the Arab Countries, South America (Peru and Bolivia), and to the Caribbean Region. ${ }^{33}$ In the final report's executive summary, WIPO highlights the scope

\footnotetext{
32 See also Halewood 1999:965-985 for an overview of conventions and committee concerned with TK and TCEs. They include the Convention on Biological Diversity and other documents approved at the United Nations Conference on Environment and Development (UNCED) in 1992, work done in the context of the ILO, and farmers' rights.

33 See http://www.wipo.int/tk/en/tk/ffm/report/final/, accessed 1 June 2011. The classification of the nine regions is taken from this report.
} 
and intention of these missions as mainly directed at the interests and needs of "holders" of TK:

The fact-finding missions (the FFMs) were designed to enable WIPO to identify, as far as possible, the IP needs and expectations of TK holders. While the needs of TK holders have been referred to in other international fora, there has been to date no systematic global exercise by international organizations to document and assess, firsthand, the IP-related needs of TK holders. As the United Nations specialized agency responsible for the promotion of the protection of IP, WIPO undertook the FFMs as part of a new programme of activities, initiated in 1998, to explore and study current approaches to, and future possibilities for, the protection of the IP rights of holders of $\mathrm{TK}^{34}$

The fact-finding missions have been a valuable resource for the committee, providing information on diverse cultural property contexts and the relation between national and international intellectual property infrastructures on the one hand and TK, TCEs, GR and social actors connected with these resources on the other hand.

Actions to establish the IGC ensued in 1999, though its creation seems to have been more unplanned than planned:

The establishment of this forum, the Intergovernmental Committee on Intellectual Property and Genetic Resources, Traditional Knowledge and Folklore, was an unintended outcome of a proposal submitted to the third session of WIPO's Standing Committee on the Law of Patents (SCP) (Dutfield 2004: 134).

The Colombian delegation at that session submitted a controversial document entitled 'Protection of biological and genetic resources' proposing linking patents relating to GR to an assurance that the resource had been acquired legally, and that this be accompanied by an access and benefit sharing (ABS) clause. Many developing countries supported this proposal, while developed countries argued against it. The dissatisfaction of these countries with how intellectual property legislation concerning cultural matters were being addressed was rooted in conflicts with pharmaceutical companies that were said to be capitalizing on traditional medicinal knowledge or concerned the misappropriation of indigenous artworks or folklore for commercial purposes. In consequence, delegations compromised to keep these issues out of the SCP and in return have a meeting specifically on issues of intel-

\footnotetext{
34 http://www.wipo.int/export/sites/www/tk/en/tk/ffm/report/final/pdf/part1.pdf, accessed 1 June 2011.
} 
lectual property and GRs. This meeting led to a proposal that was eventually taken up by WIPO's GA, which decided to establish the IGC in 2000 (ibid).

Actors and institutions from local, national, regional and international levels are involved in debating the constitution of a cultural property right in WIPO's IGC. They all bring specific expectations, desires, levels of competence and languages to the table, which both shapes and limits the field of discourse. Negotiations and talk about traditional knowledge takes place in a multitude of fora, including the CBD, the WTO, the ILO, the Food and Agriculture Organization (FAO), and even the World Meteorological Organization (WMO). This nicely illustrates the entanglement of institutions at the international level in the issues, and there are of course connections to local, national and regional organizations as well.

Actors in the IGC also not only come from a multitude of institutions and organizations, they also come from a broad range of sociocultural backgrounds, have differing political and moral views, expectations, levels of competence, intentions, or policy guidelines to follow. They hold personal as well as professional opinions and, in their subjectivities, maintain interpersonal relationships that are expressed in their performance on the international stage. Moreover, the actors at the IGC meet in various temporal-spatial constellations: in the main meeting room, in the hallway, the cafeteria, the Director-General's office, at a side-event or workshop, for lunch in an embassy, at a hotel or shopping mall, on the way from the UN headquarters in Geneva to the WIPO building. Or they may meet on a train from Geneva to Berne, all of which leads to an immense number of both formal and informal settings that are ethnographically of considerable interest. Most visible, of course, and the most accessible to closely study are the encounters in the WIPO meeting room where the sessions of the IGC take place.

Since WIPO's establishment, much effort in the IGC has been devoted to define TK and TCEs. ${ }^{35}$ WIPO, as well as its member states, is well aware that definitions influence the scope of discussions and potentially have far-reaching implications. This is both due to the specificities of international law that has to take national legislation into account, and with the interests and strategies of member states not to include too much under the labels TK and TCEs. In a document prepared by the WIPO secretariat, it therefore reads:

International IP standards typically defer to the national level for determining the precise scope of protected subject matter. The international level can range between a description in general terms of eligi-

\footnotetext{
${ }^{35}$ See the discussions in documents WIPO/GRTKF/IC/7/6 ("Draft Outline of Policy Options and Legal Elements for the Protection of Traditional Knowledge") and WIPO/GRTKF/IC/7/3 ("Summary of Draft Policy Objectives and Core Principles for the Protection of Traditional Cultural Expressions/Folklore”).
} 
ble subject matter, a set of criteria for eligible subject matter, or no definition at all. For example, the Paris Convention and the TRIPS Agreement do not define "invention". 36

As will be discussed in greater detail below, issues of definition are highly complex in the IGC. The IGC has worked through a large number of case studies and there is thus a general understanding on the part of IGC participants about what TK, TCEs and GRs are, including the social and political contexts they are situated in. But as actors carry divergent views as to the economic, political and social values of TK, TCEs and GR, there are also fundamentally divergent policy objectives in the IGC. They result in different perspectives on the IGC's subject matter. While there are specific examples for TK in the IGC, actors do not necessarily share the same views about these examples. Differences exist in terms of their social, cultural, or political valuation, as well as in the strategic implications of these views. There is an understanding at the level of information, but the way actors perceive of that information and the conclusions they draw from it are not necessarily shared. Likewise, there is disagreement about the legal classification: the specific qualities something must have to be classified as TK, TCEs or GR are disputed between IGC participants. This classification can have legal as well as political and financial implications for member states and other actors, and thus it is discussed in a controversial manner. However, the specific characteristics and terms that qualify something as belonging to these categories are mostly unclear with regard to the legal dimension. Similarly, this study will abstain from trying to define what TK or TCEs are as this would level the different perspectives and perceptions of these substantial issues of the IGC process. A discussion of divergent perspectives on cultural property is part of Chapter 5 .

Yet, there have been attempts at defining TK and TCEs, and they offer a broad overview of what belongs to these issues and what not. They convey an understanding of what actors in the IGC mean when referring to TK and TCEs. An important distinction made in the context of the IGC is that of TK lato sensu and TK strictu sensu:

As a broad characterization, TK lato sensu can be understood as 'the ideas and expressions thereof developed by traditional communities and Indigenous peoples, in a traditional and informal way, as a response to the needs imposed by their physical and cultural environments and that serve as means for their cultural identification.' TK latu sensu becomes a convenient umbrella term covering both aspects of protection of TK stricto sensu and TCEs (in this broader sense, it goes beyond 'knowledge' as such). Some objects of protection touch simultaneously upon those two distinct fields of IP, such as technical crea-

${ }^{36} \mathrm{WIPO} / \mathrm{GRTKF} / \mathrm{IC} / 11 / 5(\mathrm{c}): 20$. 
tions that have an aesthetic character. For instance, many handicrafts have a utilitarian function, having been developed with a utilitarian purpose and giving effect to a technical idea, but may acquire an additional aesthetic quality. Either because of their use in religious services and other spiritual events, or because of their general association with a culture and a community, handicrafts may become more important as a cultural expression than simply as the product of a technical idea. In this vein, handicrafts may embody TK stricto sensu or may be viewed as expressions of TK or TCEs. ${ }^{37}$

Thus, the distinction between TK and TCEs stems from the technical separation of these domains in the intellectual property system. Therefore, what is used in IGC negotiations is TK in its strict sense, consisting - according to a draft definition - of the

content or substance of knowledge resulting from intellectual activity in a traditional context, [including] the know-how, skills, innovations, practices and learning that form part of traditional knowledge systems, and knowledge embodying traditional lifestyles of indigenous and local communities, or contained in codified knowledge systems passed between generations. It is not limited to any specific technical field, and may include agricultural, environmental and medicinal knowledge, and knowledge associated with genetic resources. ${ }^{38}$

Similarly, there have been attempts by the IGC to outline the components of TCEs, as in this enumerative definition from "The Protection of Traditional Cultural Expressions/Expressions of Folklore: Overview of Policy Objectives and Core Principles":

'Traditional cultural expressions' or 'expressions of folklore' may be understood as including productions consisting of characteristic elements of the traditional cultural heritage developed and maintained by a community, or by individuals reflecting the traditional artistic expectations of such a community. Such productions may include, for example, the following forms of expressions, or combinations thereof: (i) verbal expressions, such as folk tales, folk poetry and rid-

\footnotetext{
${ }^{37}$ WIPO/GRTKF/IC/7/6, Annex I:22.

38 WIPO/GRTKF/IC/18/5. This definition is taken from a "Glossary of Key Terms Related to Intellectual Property and Traditional Knowledge" (WIPO/GRTKF/IC/19/INF/8:23) that is also available for TCEs (WIPO/GRTKF/IC/19/INF/7) and GRs (WIPO/GRTKF/IC/19/INF/9). These documents gather comments and text passages from other texts produced by the IGC in order to give an overview of the key terms as used in the context of the IGC. It is, however, important to note that these are not consensual definitions agreed upon by member states.
} 
dles; aspects of language such as words, signs, names, symbols and other indications; (ii) musical expressions, such as folk songs and instrumental music; (iii) expressions by action, such as folk dances, plays and artistic forms or rituals; whether or not reduced to a material form; and (iv) tangible expressions, such as: (a) productions of folk art, in particular, drawings, designs, paintings, carvings, sculptures, pottery, terracotta, mosaic, woodwork, metalware, jewelry, basket weaving, handicrafts, needlework, textiles, carpets, costumes; (b) musical instruments; (c) architectural forms. ${ }^{39}$

There is thus an understanding of what TK and TCEs are for the purposes of discussions in the IGC that actors can refer to - but the passages quoted above need to be understood for what they are: efforts to summarize key terms. These are not consensually agreed-upon codifications by WIPO participants.

For this reason, these "definitions" are an important strategic component in negotiations that are used by actors to foster their interests. Member states do not lack the competence to agree on definitions, ${ }^{40}$ but rather are unwilling to commit to definitions that might have unwanted and unforeseeable implications and consequences. In other words, definitions are matters for strategy, negotiation, and diplomacy.

WIPO's IGC now brings together 184 nation-states and a significant number of NGOs and ILCs, and focuses on developing legal frameworks for intangible cultural resources within the intellectual property regime. The founding of the committee was influenced by increasing pressure from developing countries and indigenous groups on WIPO and the UN (Hafstein 2004: 301).

The expectations of member states and observers regarding the results of this process could not be more diverse. Western industrial nations are largely satisfied with the current state of copyright and patent regimes and not very interested in changes or in creating a new legal instrument for intellectual property. The current system works to their advantage, as patented and copyrighted works are protected not only at the national, but at the international level as well.

This is not true of the resources under negotiation in WIPO's IGC. No vigorous instruments exist in international intellectual property law that can combat the transnational misappropriation of cultural resources. Therefore, countries which have large indigenous populations and a broad range of TK and TCEs exert pressure to create legally binding instruments at the international level that can protect

\footnotetext{
${ }^{39}$ WIPO/GRTKF/IC/7/3, Annex I: 4.

40 The involvement of anthropologists and folklorists in the committee, moreover, could have helped in drafting working definitions, were it not for a lack of willingness on the part of member states to accept such involvement.
} 
or compensate the holders of TK - or be financially or politically beneficial for the country itself.

Despite the lack of an agreement or legal instruments, over the course of its existence the IGC has produced a number of important documents on TK and TCEs. The most important are the "Policy Objectives and Core Principles" for the protection of TK and $\mathrm{TCEs}^{41}$ prepared by the WIPO secretariat and presented for the first time in November 2004. These have been revised and commented on numerous times and are still referred to in discussions. More importantly, they have been used by some member states - for instance in Latin America - to draft national legal instruments. They outline central aspects both for policy frameworks and for defining the legal scope of a possible instrument and have been distilled from other documents, comments and information in the IGC process. Related are the "Lists of Issues" for TCEs and TK, ${ }^{42}$ which collect central aspects of negotiations and commentary. These lists, for example, include the question of definition, the duration of protection, the beneficiaries of an instrument or possible sanctions against misuse. ${ }^{43}$ The issues outlined therein are a guideline for current negotiations as well, as they clarify the scope of negotiations and provide an overview of problems to be solved. ${ }^{44}$ Another set of important documents are the gap analyses $^{45}$ on TK and TCEs, ${ }^{46}$ analyzing whether legal instruments at the national and international level provide for the protection of cultural property or not. The resulting discovery of gaps in protection was then to be considered - with regard to intellectual property - in the legal instrument to be drafted by the IGC. Finally, there are the texts that are the current focus of the IGC. The inter-sessional working groups (IWG) on TK, TCEs and GR were, according to a decision prepared by the IGC and taken by the GA, to be held with the involvement of experts. Their task was to develop draft texts on the basis of the existing work of the IGC, taking into account the lists of issues as a guideline. These texts have been presented to the IGC as "Draft Articles on the Protection of Traditional Cultural Expressions prepared at IWG 1" and "Draft Articles on the Protection of Traditional Knowledge prepared at IWG 2". ${ }^{47}$ Both texts are used as a basis for drafting agreements on the protection of cultural property in the current sessions of the IGC.

\footnotetext{
${ }^{41} \mathrm{WIPO} / \mathrm{GRTKF} / \mathrm{IC} / 7 / 5$ for TK and WIPO/GRTKF/IC/7/3 for TCEs.

42 WIPO/GRTKF/IC/11/5(a) for TK and WIPO/GRTKF/IC/11/4(a) for TCEs.

${ }^{43}$ See http://www.wipo.int/tk/en/igc/issues.html, accessed 1 May 2011.

${ }^{44}$ The lists have been complemented by "factual extractions" gathering comments, notes and addenda by member states (WIPO/GRTKF/IC/12/5(B) for TK and WIPO/GRTKF/IC/12/4(B) for TCEs).

${ }^{45}$ See http://www.wipo.int/tk/en/igc/gap-analyses.html, last accessed 1 May 2001.

${ }^{46} \mathrm{WIPO} / \mathrm{GRTKF} / \mathrm{IC} / 13 / 5$ (b) Rev. for TK and WIPO/GRTKF/IC/13/4(b) Rev. for TCEs.

47 WIPO/GRTKF/IC/18/7 for TK and WIPO/GRTKF/IC/17/9 for TCEs. Currently, there are no such draft articles on GR.
} 
While the immense number of documents prepared by the IGC since its inception in 2001 has not produced tangible outcomes at the international level, these texts show the amount of work completed by the IGC and that the committee is moving forward, although at a very slow speed and with many member states stalling negotiations. In terms of international treaty negotiations, the IGC is still a relatively young forum. Apart from that, some member states see the slow speed of negotiations already as success: the work of the IGC, however slow it may be, shows that they are working on issues of GRTKF.

Numerous capacity-building initiatives, national and regional projects, ${ }^{48}$ and bilateral agreements draw from the work of the IGC. These aspects have to be taken into account when assessing the work the IGC has achieved so far.

IGC participants come from a broad range of sociocultural and linguistic backgrounds. Thus, the committee is challenged not only by different strategic agendas, but also a by multitude of perceptions of what "culture", "property" and "community" mean. While NGOs and indigenous communities are admitted only as observers and have no voting power, they nonetheless frequently partake in the discussions. This multiplies perspectives, increases ambiguity and reduces the degree of shared understanding of terminology and its interpretation among the different actors. A prime example for this is that after more than ten years of negotiations, there is still no consensual or binding definition of "traditional knowledge" or "traditional cultural expressions". Thus, it is appropriate to take a closer look at the constellation of actors in order to understand the different interests as well as perspectives that actors have in the context of TK, TCEs and GR.

\section{Actors and Alliances}

Actors of the WIPO IGC process are what Hymes termed 'participants' in his SPEAKING-model. The unit of analysis of the ethnography of communication is the speech event. However, as for instance the component 'setting' illustrates, the analysis of communicative events is not limited to the actual conversation or communicative exchange. The setting of a speech event also includes its broader context, like negotiations on TK in the CBD in the case of the IGC. Similarly then, the participants of the IGC's communicative events are not only those present during the sessions and meetings, but also external actors influencing what is happening at WIPO. This includes national government agencies determining the

\footnotetext{
48 The "Swakopmund Protocol on the Protection of Traditional Knowledge and Expressions of Folklore" prepared by the African Regional Intellectual Property Organization (ARIPO) and adopted by the Diplomatic Conference of ARIPO at Swakopmund (Namibia) on August 9, 2010, is one of such initiatives in national and regional contexts. The text of the protocol can be found at http://www.aripo.org/images/Swakopmund_Protocol.pdf, accessed 1 June 2011.
} 
position a delegation is going to take during negotiations as well as numerous civil society and industry organizations working in local and international contexts on the protection of GRTKF. Moreover, the work of legal scholars as well as of anthropologists influences the negotiation process at WIPO.

The range of actors who participate directly and indirectly can be grouped into five different categories: WIPO staff, member states, IGOs, NGOs and ILCs.

\section{WIPO Staff}

The IGC is part of WIPO's Global Intellectual Property Issues Division, and consists of three sections: the "Traditional Creativity, Cultural Expressions and Cultural Heritage Section", the "Genetic Resources, Traditional Knowledge and Biotechnology Section" and the "Life Science and Public Policy Section", each headed by separate individuals. The first two sections specifically deal with TK, TCEs and GR, and administer issues of the IGC. These sections have five professionals, three secretaries and two administrative assistants each. ${ }^{49}$

The tasks of WIPO's secretariat are manifold. It has to compile reports, working documents and other publications, prepare and organize meetings such as the IGC, present the activities of the IGC in numerous fora, and participate in workshops, including capacity-building programmes, and other activities relating to the protection of TK, TCEs and GR. It also provides professional assistance and expertise on issues discussed in the IGC to various actors, and its work is aided by WIPO's translation unit in preparing documents in WIPO's working languages (simultaneous translation during the sessions is provided by external interpreters).

Thus, for the IGC, the WIPO secretariat plays an essential role in both organizing and facilitating negotiations as well as furthering the IGC process in accordance with its mandate. Furthermore, the secretariat helps ILCs participating in the IGC meetings, including organizing their accommodation and arranging other organizational matters. In numerous cases, IGC participants used to work at WIPO, or where WIPO staff used to be part of a member state's delegation.

\section{Member States}

Currently, WIPO has 184 member states..$^{50}$ Regional groups include (1) the African Group; (2) Group B, consisting of industrialized countries like the U.S., Japan, New Zealand, the European Union, or Australia ; (3) the Asian Group; (4) the European Union; (5) the Central European and Baltic States; (6) the Latin American and Caribbean Group (GRULAC); and (7) the Caucasian, Central Asian and Eastern European Countries. China is considered a regional group of its own, and

\footnotetext{
${ }^{49}$ Author's notes from an interview with a WIPO staff member.

${ }^{50}$ See http://www.wipo.int/members/en/, accessed 1 June 2011.
} 
sometimes a group of least-developed countries (LDCs), with its own regional group coordinator, is in attendance. In 2007, WIPO adopted a "development agenda" 51 with 45 recommendations that, among other things, propose a stronger involvement of LDCs. It is possible these recommendations may lead to changes in the current framework of regional groups. Only member states are allowed to vote or make proposals in drafting text on their own in IGC negotiations, ${ }^{52}$ however, and thus they are the most important group of actors. ${ }^{53}$

As noted above, developed countries, such as the EU or those in Group B, are largely satisfied with the current state of the intellectual property system and generally not interested in changing existing legislation or adding new legal instruments that would change intellectual property and cultural property laws. In personal conversation, they are open about this position, and other delegates as well as NGO and ILC participants are aware of it as well. Conventional patented and copyrighted works are protected not only at the national, but at the international level as well by treaties administered by WIPO or the WTO. ${ }^{54}$

This is not true for cultural property. No binding instruments exist in international intellectual property law to prevent the transnational misappropriation of cultural resources. Therefore, countries with large indigenous populations or a broad range of TK, TCEs or GRs (e.g., India, Brazil, Peru, many African states) want to see a legally-binding agreement adopted at the international level that protects these resources, establishes access to and regulates the benefits of that access, or established prior-informed consent mechanisms. Furthermore, other developing countries not directly interested in the protection of intangible cultural property support these processes as they influence broader power relations within the UN system. In such cases, discussions of TK and TCEs are used as political leverage for other issues.

Developing countries also tie the discussions about traditional knowledge and intellectual property to broader negotiations on the development agenda. They claim development is itself a human right and argue that discussions of intellectual property need to shift from a technical exercise of minimally adjusting the existing intellectual property system to a broad-minded debate on the role intellectual property plays in development, human rights, and traditional knowledge. From

\footnotetext{
${ }^{51}$ See http://www.wipo.int/ip-development/en/agenda/overview.html, accessed 9 June 2011.

${ }^{52}$ NGOs, ILCs, and other participants can make proposals as well, but they have to be supported by at least one member state to be kept in the text.

${ }^{53}$ As in the rest of the UN, WIPO only acts according to the will of its member states. For that reason, its secretariat only compiles documents or arranges fact-finding missions on behalf of member states. In voting procedures, each member state has one vote, and a simple majority is sufficient to take decisions - but in the IGC, no votes have been taken to this point. Rather, decisions in the committee are reached by consensus.

${ }^{54}$ Especially the "Agreement on Trade-Related Aspects of Intellectual Property Rights" (TRIPS).
} 
their perspective, reshaping the intellectual property system is less a task for intellectual property lawyers and diplomats, and more a global question of justice and human rights.

So one can differentiate between countries interested in speeding the process up and trying to find agreement beneficial to their interests on the one hand, and countries trying to slow the process down and prevent the adoption of legal instruments that would be disadvantageous for them. ${ }^{55}$ However, such countries are interested in keeping TK, TCEs and GR issues inside the IGC, as conducing these discussions in other fora would be counter-productive for their strategies. In that sense, the IGC confines the debate on GRTKF to one venue that, at present, does not promise any tangible outcomes in the near future. In the WTO, the CBD and other international organizations, stalling delegations can refer to the negotiations at WIPO, arguing that these issues do not have to be discussed: a norm-setting track for addressing intellectual property aspects of TK, TCEs and GR already exists. Simultaneously, no substantive decisions are being made at the IGC, which is a beneficial situation for developed countries. Thus, speeding up delegations often threaten to leave the negotiation table and continue the discussions on GRTKF in other more promising fora.

Yet, it has to be considered that a simplifying dichotomy of "speeding up" and "stalling" delegations is an abstraction to characterize far more complex and multilayered processes. Insofar as there are tendencies towards this dichotomization, coalition-building processes in which national delegations are persuaded to join one or another side in exchange for concessions in other fora, may reinforce it. To give an example, Egypt has a notably strong voice in WIPO IGC negotiations on traditional knowledge and folklore. Yet, in private conversation, one high-ranking Egyptian delegate made clear to me that his country has no interest in the protection of TK or TCEs whatsoever - it is not on the domestic agenda, nor does it play a big role in diplomacy. Yet, in negotiations, the Egyptian delegate makes drastic statements and demands, increasing the pressure on the stalling delegations as much as possible.

The delegate explained this position by noting that Egypt regards the current UN system as unbalanced and disadvantageous to developing countries. So to increase the pressure on industrialized nations, negotiation tokens from WIPO's IGC are used to try to bring about changes in the UN system, including in neighboring fora like the WTO or CBD.

The inclusion of human rights arguments is one way to add weight to the negotiating position of developing countries. The debate on TK is modified to make it compatible with broader discussions of the current UN system, the conflict between North and South globally, and to raise issues of global justice. By shifting

\footnotetext{
55 This is a decriptive distinction between different interests of member states and not a normative dichotomy.
} 
the perspective on the substantive issues, traditional knowledge becomes no longer just knowledge of a special quality or type, but a potential right. Terminology and taxonomy are altered ever so slightly, and since there are no binding definitions of central terms, the subtle shift can have weighty implications. Similarly, questions of procedure are re-phrased. By conceptualizing the issue on the table as a matter of human rights, a relatively slow process is transformed into an urgent affair that needs to be taken care of immediately. The substantive questions about the global protection of TK and TCEs are taken out of the bounded negotiation room into a larger context of concern to civil society.

The building of coalitions and the relation between speeding up and stalling delegations is thus a dynamic process that is negotiated both in front and back rooms in and beyond the IGC process. Some of the stages where it is negotiated are described in the next section, and some of the signaling mechanisms related to language use and regulating these processes will be explored later. However, the dichotomy between stalling and speeding up is constantly reinforced by actors referring to "we" and "them" as an expression of these basic stances.

Member states are represented either by diplomats or by experts from ministries and institutions dealing with issues of GRTKF and the intellectual property system. As for diplomats, a distinct habitus and certain habits come with the profession of envoys and state representatives. Books have been written on this, most notably probably Albert Cohen's “Belle du Seigneur". Having worked as a diplomat in the Geneva UN apparatus himself, Cohen digresses over pages on the daily doings of his semi-biographical protagonist Solal, who is usually terribly busy, yet achieves nothing definite or conclusive at the end of the day. On another literary account by a Geneva based diplomat involved in the IGC, it reads:

And I won't tell about diplomats in meetings or cocktails which constitutes their natural habitat. I mean generally. Manners. Jargon. Odour. Gazes. It is true that every single profession has its own characteristics and vocabulary, but in the case of diplomats, it's like behaviour would precede them. You can smell them. They recognize each other as well, and behave accordingly. If they would just lost their diplomatic attitude in a distracted moment of normality; if they would just recover their humanity for a while, they would immediately recover their diplomatness at the simple observation of another of their kind.

It is certainly true that there is a distinct habitus or set of competencies that is required to act on the diplomatic stage without causing any damage, and members of national delegations in WIPO's IGC are very frank when it comes to admitting that it takes a lot of time to accustom to these rules of behavior and, more importantly, 
language: initially, meetings are only observed by "newcomers", then statements are drafted and coordinated with superiors, passages are copied and pasted from previous documents, advice is sought by more experienced colleagues, and it is sometimes only after years that one is allowed to act on his own in the shaping of diplomatic events.

Yet, not all members of national delegations in the IGC are professional "diplomats." While some live and work in the permanent missions in Geneva and attend numerous meetings in the UN system, many are send to Geneva by their ministry or agency in their capacity as experts on intellectual property. To name a few, there are physicists working in their national patent office, lawyers primarily dealing with industrial property issues, legal scholars or civil servants from ministries of justice. It is thus not only diplomats acting on this stage, but also a large number of professionals from other fields. This leads to an observable difference in competence: while some delegates are able to deliver their statements off-the-cuff and are well familiar with the committee's procedure, others take more time to draft their statements. To some extent, the experience of a delegate is indicative of how important the IGC negotiations are for a given country.

Intergovernmental organizations

International and intergovernmental organizations (UNESCO, SCBD, WTO, FAO, the African Union, ARIPO) participate in the IGC as observers. While they do not have the right to vote, from time to time they make statements, mainly pertaining to the work these organizations undertake that relates to cultural property. As the activities of some of these IGOs and WIPO overlap, such as the work of the CBD on TK or ARIPO's protocol on the protection of TK, their involvement reveals the interrelations between different fora and actors.

\section{Non-Governmental Organizations and Indigenous and Local Communities}

Currently, 188 organizations are accredited as ad hoc observers to the IGC, many of which are ILCs. ${ }^{56}$ Their scope is broad, and includes industry organizations such as the Agency for International Trade and Cooperation (AITIC), the Intellectual Property Owners Association (IPO), the International Chamber of Commerce (ICC) or the World Trade Institute (WTI). Civil society organizations, such as the Center for International Environmental Law (CIEL), the Third World Network (TWN), the Berne Declaration or Knowledge Ecology International (KEI), monitor the proceedings at WIPO, comment on the process, prepare publications and workshops (informing other NGOs about the current status of the IGC and the relations to other fora) and of course lobby for their cause. Other observers, in-

\footnotetext{
${ }^{56}$ For a full list of NGOs and ILCs accredited to the IGC, see http://www.wipo.int/tk/en/ngopar ticipation/, accessed 4 May 2011.
} 
cluding those from research institutions or scholarly organizations, are also accredited, and among them one finds the American Folklore Society (AFS), the Societé Internationale d'Ethnologie et de Folklore (SIEF), the Göttingen Research Group on Cultural Property (RGCP), the Max Planck Institute for Intellectual Property and Competition Law or the Queen Mary Intellectual Property Research Institute (QMIPRI). Their representatives in part contribute their expertise to the process.

Numerous indigenous and local communities are accredited as well, including the Tulalip Tribes of Washington Governmental Affairs Department, the Swiss Society for Ethnomusicology (CH-EM), the Russian Association of Indigenous Peoples of the North (RAIPON), the Inuit Circumpolar Council, the Grand Council of the Crees (Eeyou Istchee) (GCCEI) or the African Indigenous Women Organization (AIWO). Some, though not all, of these ILCs are also part of the indigenous caucus, a bloc of organizations coordinating the interests of indigenous groups. They hold meetings during the IGC sessions, communicate via email between sessions and sometimes give statements on behalf of their members in the main plenary.

As holders of TK and TCEs, ILCs participating in the IGC deserve special attention. At the thirteenth session of the IGC in Oct. 2008, held in Geneva, approximately 30 representatives of indigenous and local communities were present. Prior to attending IGC meetings "on behalf" of an organization or community, be it non-governmental, indigenous, local or cultural, a mandate has to be given to the representative of that community or organization by their "constituency". To some degree, the WIPO administration has to ensure the validity of representational claims.

This administrative requirement sets a process of formalization into motion. ${ }^{57}$ In deciding to participate in an international forum like WIPO and the IGC, an ILC must formally apply as an organization, include a description and its main objectives, and make available "the name and title of the organization's representative at the IGC sessions" ${ }^{58}$ Framing communities as organizations with a specific telos and concrete objectives requires a certain amount of reflexivity not only about socio-cultural dynamics within the community, but also with regard to its localization in global processes.

The "enforced production" of goals of a group rigidifies value systems in written form, as this requires singling out certain aspects that appear to be especially valuable and leaving out others that might otherwise conflict with the consistency of the argument. Social and cultural conflict is "smoothed away" with a view to present oneself at one's best. These processes of inventing objectives and posi-

\footnotetext{
${ }^{57}$ This also entails processes of demarcation - creating boundaries between social groups by formalizing membership categories - and transformation - modifying social and political structures due to external requirements.

${ }^{58}$ http://www.med.govt.nz/templates/MultipageDocumentTOC_40925.aspx, accessed 1 June 2011.
} 
tioning values seldom have a direct effect on the communities. But as they draw on existing discussions and discourses about intellectual property and culture, and aim to create a nexus to global negotiating processes, they embed local practices in global discourses. They thus also strategically align communal interests with global policies. In some cases, this can give strategic leverage in national legislation when arguments from WIPO discussions are employed by ILCs in negotiating with state officials in their countries of origin. The IGC is thus at times used strategically to foster the ends of ILCs in other contexts. This particularism can be against the benefit of IGC discussions.

At the same time, drafting such applications and objectives, and thereby conceptualizing the respective communities as political subjects requires actors who are competent to draw from these discussions both linguistically and in terms of content. Put differently, ILC actors need to be able to instrumentalize their arguments in the appropriate jargon. WIPO's IGC, the CBD, and the United Nations Permanent Forum on Indigenous Issues (UNPFII) all use distinct terminologies, and their participants all avail themselves of specific styles and patterns of argument. To communicate one's interests and perceptions of effectively, one has to be able to reproduce the requisite linguistic registers.

The "self-presentations" of NGOs and ILCs are available in electronic form on WIPO's homepage. In terms of structure and outline, most are near-identical, as the WIPO secretariat provides a list of required information and edits the information received to fit their formatting of official documents. Looking at the content and length of descriptions, however, one finds very large differences. Some NGOs and ILCs only provide a one-sentence summary of their activities and objectives, others delineate their policy objectives and arguments in great detail. While it is unclear to what extent these descriptions and self-representations are read or have an impact on negotiations, the information provided reveals a great deal about differing levels of competency among these groups, as well as different self-perceptions and constructions of community as a subject in a global policy-making process.

This relates to broader questions of expertise with regard to substantial and procedural issues in the IGC. While some IGC representative are professional lawyers specialized in intellectual property who have attended numerous WIPO meetings and participate in other fora, others lack such ability or experience. However, there are programmes like the UN's Indigenous Fellowship Programme (IFP) ${ }^{59}$ which aim to strengthen such competencies. WIPO and the IGC have also created a voluntary fund ${ }^{60}$ supported financially by the member states, to ensure representatives of ILCs can take part in the IGC. The voluntary fund also requires a formal application and the nomination of a representative. Thus, an informal or formal

\footnotetext{
${ }^{59}$ See http://www2.ohchr.org/english/issues/indigenous/fellowship.htm, accessed 15 April 2010.

${ }^{60}$ See http://www.wipo.int/tk/en/ngoparticipation/voluntary_fund/, accessed 4 July 2011.
} 
mechanism must be in place in the ILC to nominate a representative, an expert or a delegate who is seen fit to act on behalf of the community. Upon nomination, WIPO requires a declaration as well as a resume from the representative, and a statement from the ILC itself. An advisory board consisting of members of ILCs as well as delegates from member states then must decide about the nominations of ILCs that have been submitted. While indigenous groups thus are becoming more and more involved in international processes (Cadena 2007) and their voices more loudly heard in negotiations, they are not equally involved in the decisionmaking process: when text is drafted or decisions on future work are taken, proposals by ILCs must be supported by a member state. Otherwise, they are deleted. Similarly, if it came to a vote on an international instrument on GRTKF, ILCs would not be allowed to participate.

What can furthermore be observed are member states speaking for ILCs, arguing to represent their interests. Considering the problematic relation between the state and indigenous population in many countries, this position is critiqued by ILC participants at WIPO as well. Yet, many member states make use of this argument of representation to bolster their position in negotiations.

\section{The Multiplicity of Communicative Events}

The main plenary is of central importance to the IGC, yet there are also other communicative events in the IGC process. There are coordinating meetings of the regional groups, consultations with the session's chair or WIPO's Director-General, small groups for drafting text, inter-sessional working groups, the indigenous panel and caucus, numerous instance of hallway or cafeteria talk, forms of electronic communication as well as other fora, venues and committees where IGC participants are involved and where IGC issues are discussed. ${ }^{61}$

Regional group meetings were typically held on the morning of the first day of an IGC and each subsequent morning and/or evening. Sometimes, additional coordination meetings were held during the lunch break, contingent on the stage of negotiations. In a more informal way, at least in the meetings observed, actors discussed the strategy to be used during the main session, and coordinated tasks such as the drafting of shared statements or written proposals. Usually, the intent behind various strategies were openly debated. However, the underlying rationale behind the general stance (for Group B and the EU, stalling, as delegates confirmed in personal conversation) was only at times made explicit, as actors seemed

\footnotetext{
${ }^{61}$ For fieldwork, this poses the problem of access to these meetings. While I had access to regional group coordination meetings of Group B and the EU at IGC 12 and to the meetings of the indigenous caucus, it was not possible to observe the meetings of the African or Asian group, nor to attend inter-sessional meetings by regional groups. In the latter case, however, interviews as well as document analysis and participant observation in the main plenary and in some group meetings allowed a broad picture to be drawn that was sufficient for the purposes of this study.
} 
to agree upon it quasi-automatically by their membership to Group B or the EU.62 Thus, it was discussed how to edit texts to avoid unwanted implications, how to react to statements and proposals made by delegations who opposed them, and what to do in specific situations.

Apart from the regional group coordination during IGC sessions, member states sometimes also gather between IGC meetings to decide on strategy and draft or comment on documents. An African Group proposal on future work ${ }^{63}$ was prepared during a meeting of African member states in Durban, and a member of the WIPO secretariat was present at that meeting as an advisor. A statement by the EU had been drafted at a coordination meeting in Brussels and was then presented at the IGC. The indigenous caucus can in a way also be understood as a regional group of its own, although less institutionalized and legitimated than the other groups. Not all representatives of ILCs agree with the format or share the views expressed in it. It is interesting to note that the caucus has adopted many of the communication rules and conventions of the UN system. ${ }^{64}$

At times, and then usually in critical or deadlock situations, regional group coordinators meet with each other and the session's chair for informal consultations to try to find solutions. In some cases, these meetings take place together with WIPO's Director-General, who facilitates the exchanges. In recent meetings, "friends of the chair" - a small group of regional group coordinators and other high-level representatives - have gathered to discuss procedural and substantive issues. While the purpose is to facilitate consensus, such informal consultation has been criticized, especially by ILCs. While the result of these meeting might be positive, the lack of transparency and access means that some views are not being heard.

In more recent plenary sessions starting with IGC 14 in mid-2009, text has been edited in the main plenary. A document was projected onto the screen behind the dais and delegations commented on the listed issues. As this has proven rather inefficient, small and openly accessible drafting groups have been established in Room B of WIPO's main building instead. Due to the materiality of this room and a more crowded "feeling", the atmosphere observed during such drafting sessions was less informal than in the main plenary. There was more direct communication and a more vivid exchange of views and reactions on both the negotiated text and the activities of other delegations.

Another communicative event of the IGC are the inter-sessional working groups (IWGs) on TK, TCEs and GR. Meeting between two IGCs and consisting of only a few representatives from each regional group, along with some observers

\footnotetext{
${ }^{62}$ The case of the EU is special, as IP issues are generally coordinated on the EU level rather than on the national level.

${ }^{63} \mathrm{WIPO} / \mathrm{GRTKF} / \mathrm{IC} / 13 / 9$.

64 The chair of the indigenous caucus at IGCs 12 to 17 argued it is necessary to "play by their rules" in the plenary as well as in other communicative events in order to be heard (author's notes, IGC 14).
} 
and experts, they have proven successful at producing text on the three substantive issues. These texts are then brought back to the IGC and used as the basis for further discussions. Communication via email and WIPO's website is another form of inter-sessional work. Delegations and observers are encouraged to submit comments and proposals this way, and the large number of comments on the homepage indicates this is done frequently. ${ }^{65}$

The indigenous panel meets at the beginning of an IGC session. It is chaired by an indigenous representative, and a number of representatives of ILCs attend: its aim is to communicate examples of misuse and misappropriation in specific contexts. The format has been criticized by many IGC participants, as the quality and germaneness of presentations vary significantly. A central criticism heard especially from the delegations of member states is that the problems and grievances described in the indigenous panel are not related to what is being negotiated in the IGC. Instead, one observes and hears many lengthy statements about social and political inequalities, often without a direct connection to the intellectual property system at the international level.

Additionally, other fora, venues and committees outside WIPO have to be mentioned, as well as the numerous cases of hallway and cafeteria talk. The latter sometimes facilitates exchanges that otherwise would not happen, as when a delegate of an openly stalling country finds himself by chance at a table together with a proponent of finding quick agreement on the protection of TK, TCEs and GR.

To summarize, WIPO's IGC committee is closely related to other international fora, both with respect to the substantive issues and to the actors participating in negotiations. NGOs, ILCs, and representatives of member states often participate in more than one international organization. GR, or TK and TCE-related issues are negotiated in fora like the CBD or WTO's TRIPS committee. Discussions of "biopiracy" and "bioprospecting" as well as questions about "Access and Benefit Sharing" (ABS) and "Prior Informed Consent" (PIC) by ILCs are part of the policy environment in which cultural property is discussed. Political anthropologists have conducted micro-studies on these topics, connecting local processes with global discourses (Hayden 2003a, 2003b, 2005, 2007; Coombe 2008) and these feed back into IGC discussions. Studies of the role of NGOs in international negotiations (Fisher 1997, Abélès 2008) and at the local level (Schuller 2009) furthermore provide links for analyzing their influence on IGC processes. The anthropological debate on human rights, especially in the context of international law (Merry 2006a) and international organizations (Merry 2006b), also is part of this debate, as human rights are discussed in the IGC as well. The edited volume on "Cultural Heritage and Human Rights" (Silverman and Ruggles 2007) provides an overview of this connection. The notion of indigeneity as used in the IGC, and as reflected in the term "indigenous and local communities", is highly problematic, as Lee

\footnotetext{
${ }^{65}$ See http://www.wipo.int/tk/en/resources/, accessed 2 May 2011.
} 
(2006) and Cadena (2007) describe. The term contested and has neither been defined in WIPO nor in the CBD or the Declaration on the Rights of Indigenous Peoples (UNDRIP). Yet, it is used frequently in negotiations.

Political and linguistic anthropology also emphasizes the role documents play in negotiations and what their influence is on political and social realities (Riles 1999, 2006; Brenneis 2006). Unique to the WIPO process is a very extensive, and transparent, documentation of discussions, spanning from session reports and statements by member states to the written self-presentation of organizations.

At the time of writing, WIPO's 49 $49^{\text {th }}$ GA in August 2011 is likely to renew the IGC's mandate, and state its intent to continue its efforts to draft texts for the protection of TK, TCEs, and GR. The drafts for TK and TCEs have, according to IGC participants, made substantial progress:

According to Committee Chair Philip Owade of Kenya, most of the substantive work on the texts has been done, as of this morning. After the first reading of the text early this week, Owade designated facilitators to conduct informal meetings on the three subject matters of the IGC. [...] The texts issued by those facilitators still include options and policy issues, but "they are much cleaner," he said. Those texts are expected to be taken forward to the next session of the IGC. (IP Watch, 22.07.2011) ) $^{66}$

Additionally, a group of "like-minded" developing countries, including Algeria, Angola, Bangladesh, Colombia, Egypt, India, Indonesia, Malaysia, Myanmar, Namibia, Pakistan, Peru, South Africa, Tanzania, Thailand and Zimbabwe "released proposals for the draft articles on the protection of TCEs, TK and GR", ${ }^{67}$ signaling a consensus was in reach. However, one may assume that the stalling delegations are not likely to agree with these proposals, especially since the GR draft is not very far along and some member states have already called for more discussion to clarify open questions. It remains open if and when an international legally binding instrument on TK, TCEs and GR will be established, let alone adopted and implemented by WIPO member states.

\footnotetext{
${ }^{66}$ See http://www.ip-watch.org/weblog/2011/07/22/traditional-knowledge-folkore-treaty-texts-stilladvancing-at-wipo/, accessed 29 July 2011. The facilitator's text on TCEs is available at http:// www.ip-watch.org/weblog/wp-content/uploads/2011/07/IGC-Facilitator-text-TCEs-22-July-2011.p df, accessed 29 July 2011; the text on TK at http://www.ip-watch.org/weblog/wp-content/uploads/ 2011/07/IGC-facilitator-text-on-TK -2nd-draft-22-July-2011.pdf, accessed 29 July 2011.

${ }^{67}$ See http://www.ip-watch.org/weblog/2011/07/22/traditional-knowledge-folkore-treaty-texts-still -advancing-at-wipo/, accessed 29 July 2011. The text includes links to the three proposals.
} 


\title{
The Pragmatics of IGC Negotiations
}

\author{
We're just trying to make the text coherent here and will not speak about meaning. \\ Chair of a Drafting Meeting at IGC 17, December 2010
}

What communicative strategies do actors choose, in which situations and to what ends? As outlined above, strategy or the strategic use of language in the case of WIPO IGC negotiations does not necessarily coincide with intentional communicative behavior. ${ }^{68} \mathrm{At}$ times, what we are dealing with is the unconscious, intuitive or habitualized ${ }^{69}$ use of pragmatic strategies, or in other words, of code switching. ${ }^{70}$ In other instances, however, the intention (and intentionality) behind thinly veiled indirect speech acts is very obvious. Furthermore, actors who use indirection are,

\footnotetext{
${ }^{68}$ See Brenneis 1988:228-229 for a brief discussion of the role of a speaker's intention or how strategic behavior is conceptualized in linguistic anthropology studies about meetings and negotiations. See also Duranti 1988, 1993, 1999b, 2006 for a discussion of the notion of intentionality in linguistic anthropology.

${ }^{69}$ See Mertz 2007 for examples of how law school students acquire communicative competence for legal argumentation: "I would argue that the invisibilities and silences that emerge during the inculcation of legal language in law school classrooms become hardened and habitual through multiple means in the administration of justice (linguistic and nonlinguistic, to be sure, but at least one of the linguistic means is the core of formal metalinguistic structuring outlined in this volume). In the process, it becomes less relevant what a student's background is, for once someone has thoroughly internalized the metalinguistic system of legal reasoning, she or he will begin to habitually marginalize some aspects of social context and morally grounded reasoning." (Mertz 2007:228)

${ }^{70}$ For code switching, the unconscious or unintentional usage of a switch is an insight discussed by Blommaert and Meeuwis 1998 and Woolard 2004 in relation to Carol Myers-Scotton's Markedness Model (Myers-Scotton 1998).
} 
at least in some cases, very open about it and there is a shared understanding of why such indirection is chosen. ${ }^{71}$ A number of different constellations that feature the relationship between direct and indirect speech can be distinguished:

(1) The intentionality of an utterance is very obvious. Speaker and audience are mostly aware of the implications and its indexical meaning. They understand the pragmatic features of the utterance and there is thus a metapragmatic awareness. In some cases, the strategy behind a given kind of utterance in a specific context is even openly discussed and assumed to be a very common strategy. Members of the audience might even call the speaker on such an utterance or indirect speech act. Within the IGC, this constellation applies to a large set of stalling tactics that, for example, call for an extended discussion of already discussed questions in order to prevent the negotiations from proceeding. In numerous cases, both speaker and audience are fully aware of the implications of the utterances and even discuss them openly.

(2) The speaker of an utterance is aware of some or all of its pragmatic features, and knows the implications the utterance can have. The audience, on the other hand, is not necessarily completely aware of the pragmatics of the utterance. They might guess or anticipate the intended implications, they might perceive the utterance as strategy, or they might understand it largely as a semantico-referential set phrase. During an IGC session, a delegation introduced a new key phrase related to the specific mode of negotiations, and called it "outcome-oriented deliberations". This raised many question in the audience as to the phrase's meaning, implications, and intended consequences. The rationale behind this new phrase to negotiate in a less binding and less telic way that differs from treaty negotiation - was later openly yet informally admitted by the delegation concerned.

(3) The speaker of an utterance is only partly aware of the indexical meaning of an utterance. The metapragmatic awareness is low and the utterance comes from a set of habitualized phrases or linguistic strategies. The audience, depending on its level of communicative competence in the specific kind of communicative event, can be aware of this and perceive it as a common strategy. Like the question of speaker intentionality, the perception of pragmatic strategies by a given audience does not necessarily need to be explicatory or fully conscious in the way that a strategy could be explained. Rather, metapragmatic awareness on part of the audience also expands to a more emotive or intuitive perception of strategies and their potential implications. A common example are references that function as "pivotal objects" for constructing implicit audience categories, such as specific keywords signaling a shared interest or social distinctions between the members of an audience. Alluding to specific sets of rights, such as human rights or the rights of In-

\footnotetext{
${ }^{71}$ See Brenneis's (1984) analysis of political discourse in a Fiji Indian community where "sweet talk" - indirect speech where political messages are embedded in religious speech - and "straight talk" the open discussion of political and organizational matters - are separated.
} 
digenous Peoples, or to specific social circumstances - least-developed countries, poverty, lack of health care - is one way to create normative linguistic dichotomies that need to be resolved by the constructed opponent who is implicitly addressed.

(4) The speaker of an utterance is unaware of its pragmatic features. However, there is a reproducible effect that correlates to such utterances, be it actual or only perceived. In such constellations, if the speaker realizes the pragmatic function of this type of utterance, his or her awareness of it can rise and it can be strategically and/or intuitively deployed. This can also mean that, unlike the speaker, the audience is aware of an indexical meaning. This constellation partly overlaps with the third one, the difference being that initially the speaker of the utterance does not reflect about its pragmatic features. Thus, new participants of the IGC often lack the competence in some areas of communicative events and employ phrases or social indexes not appropriate for a given situation, such as formally addressing someone in an informal setting: in one instance, a WIPO staff member was asked to give his evaluation of the progress of a IGC meeting to be expected. Addressing him in a very formal way similar to the main plenary session - including a "thank you" at the beginning of the question, an introduction of oneself and an explication which organization or country one is speaking for -, rather than in a "small talk"-situation did have the effect that one does not get an answer, as the social role of the staff member did not permit him to answer formally. After a rephrasing of the question by a mediator - in this case the moderator of an informational session a day before the official IGC meeting - the WIPO employee was finally able to respond in an informal way with his personal evaluation of the situation. Over the course of a meeting, participants acquire the competence to choose the right type of phrases for addressing other participants in the specific situations.

(5) Added to the speaker's unawareness, the type of utterance is not very common, and only a small part of the audience or no audience member at all is aware of its pragmatic features. This can be the case for relatively unknown phrases, proverbs or sayings that are regional or colloquial. Their implicit allusions are thus not understood by many members of the audience.

(6) The speaker of an utterance is unaware of its pragmatic features, and there is little to no effect observable. The indexical meaning of such an utterance is negligible.

Of course, this is an idealized typology of utterances, and in practice one finds intermediate stages with varying characteristics. In communicative events, it is of greater importance to look at the specific manifestations of the pragmatic aspects of an utterance, such as the degree of metapragmatic awareness of speaker and audience, the indexical meaning of an utterance, the ends, goals, and outcomes of that utterance, and how customary the type of utterance is.

The primary interest here are categories (1) through (4). These are communicative events that have the characteristics of communicative patterns with identifiable 
intended or perceived pragmatic qualities. Moreover, (4) points to emerging patterns or communicative strategies that might evolve into habitualized and strategically deployable pragmatic tokens. ${ }^{72}$ Cases of coincidental or idiosyncratic usage of pragmatic strategies will be largely neglected, as the contextualized use and interpretation of language is of interest here. Moreover, a contextualized analysis focuses on pragmatic strategies by a speaker in relation to a specific audience or a number of audiences. There are, of course, also pragmatic strategies that are effective and frequently used in contexts other than the IGC, but they are largely irrelevant for the negotiations examined here. Of interest here is an audience-focused view of communicative events and associated pragmatic strategies that takes into account the dialectics between the speaker of an utterance and his audience. ${ }^{73}$

\section{The IGC as a Speech Community}

The concept of speech community ${ }^{74}$ assumes the existence of a group of people "sharing a set of norms or regularities for interaction by means of language(s)" (Silverstein 1996: 285). This concept has been criticized for its presupposition of a shared set of norms, and for that reason, Duranti proposes

$[\ldots]$ that we take a speech community to be the product of the communicative activities engaged in by a given group of people. This definition takes the notion of speech community to be a point of view of analysis rather than an already constituted object of inquiry. (Duranti 1997: 82)

Instead of concentrating on shared norms or regularities of language use, Duranti suggests shifting the focus to the communicative activities themselves, interactive processes of communication in which "set[s] of norms or regularities for interaction by means of language(s)" (Silverstein 1996: 285) are reproduced or negotiated. This revision of the concept fits well with the characteristics of the IGC. More than one linguistic variety is represented in IGC negotiations, and the committee exists largely by virtue of its shared activities rather than by a continuous spatiotemporal coexistence, socio-political cohesion, or "culture". Not only do actors or participants of this speech community negotiate in different official languages

\footnotetext{
$72 \mathrm{~A}$ token is a particular instance of a concept, whereas type is the general concept. For the type-token distinction see Wetzel 2011.

${ }^{73}$ Don Brenneis has illustrated this dialectics using the example of musical and verbal performances in an rural Fiji Indian village (Brenneis 2009).

74 See Gumperz 1972, Patrick 2008, or the chapter on language communities in Burke 2000 for an extended treatment of the concept.
} 
(English, Spanish, French, Russian, Chinese, Arabic), requiring translation and interpretation, but they also employ different linguistic varieties or sociolects.

In its communicative activities, the IGC thus simultaneously incorporates differing linguistic communities and speech communities. ${ }^{75}$ There is an overlapping of multiple, segmented, sub-communities, either within the specific groups of the IGC, the IGC itself, WIPO as a whole, the diplomatic community in Geneva, or within a number of other groups in contact with the activities of the IGC. The indigenous caucus is an example of a speech community. Its activities are not limited to the committee meetings, yet these meetings constitute a frame of reference for the communicative activities and substantive debates held in this group. Then, NGOs such as the Center for International Environmental Law (CIEL) or the International Centre for Trade and Sustainable Development (ICTSD) not only attend IGC meetings but are also involved in activities of the WTO or events that address GRTKF issues. Their activities are not limited to the IGC, but it is a point of reference for their activities related to GRTKF. The same holds true for the diplomats and national delegates who either live in Geneva permanently or only come to IGC sessions: their activities are much broader than the IGC and cultural property. All these actors meet at least for the IGC sessions and for related communicative events between sessions. All these actors bring specific knowledge and "rules for the conduct and interpretation of speech and rules for the interpretation of at least one linguistic variety" (Hymes 1986: 54) to the IGC. As a result, a number of distinct speech communities are present at committee meetings and involved in the associated communicative processes.

Should we then take the IGC to be a single speech community? Or should we take the IGC to consist of various speech communities that meet and overlap as they are brought together during the activities of the committee? I would argue that, following Duranti, an analytic focus on the processes of interaction that foster a shared understanding and shared rules of interaction and interpretation allows for an understanding of the IGC as one overarching speech community, containing various sub-communities that reciprocally influence each other through

\footnotetext{
${ }^{75}$ See Silverstein 1996, Silverstein 1998, Morgan 1999, Burke 2000, Kamusella 2009 for discussions of the difference between speech communities and linguistic communities. The former can have more than one language, though they share norms of interpretation and interaction, and are defined by "sharing rules for the conduct and interpretation of speech and rules for the interpretation of at least one linguistic variety" (Hymes 1986: 54). In contrast, linguistic communities are understood to be "a group of people who, in their implicit sense of the regularities of linguistic usage, are united in adherence to the idea that there exists a functionally differentiated norm for using their 'language' denotationally (to represent or describe things), the inclusive range of which the best language users are believed to have mastered it in the appropriate way." (Silverstein 1996: 285). Silverstein gives the example of speakers of Arabic as a single linguistic community that contains various speech communities, defined by dialects, within it. Yet, across all these Arabic speech communities, there is an idea, held in the context of the broader linguistic community, that there is a "correct" way of using the language and interpreting it.
} 
their interaction and interpretation. The IGC constitutes a frame of reference that brings all the actors together in a shared activity, though the actors themselves have extremely different motivations, interests, and perceptions. Each group, whether the indigenous caucus, the NGOs, or the diplomats make reference to the IGC negotiations as a set of shared activities. Given their strong co-dependence and interrelation, it would make little analytical sense to view the IGC as a set of connected speech communities rather than as an "umbrella community" that is - for the purpose of IGC negotiations - the central point of reference for participants in the overarching speech community. From this viewpoint, the IGC is as a speech community the result of a dynamically constituted group of social actors from various backgrounds. All the participants contribute different sets of expectations, presuppositions and rules for linguistic conduct and interpretation. In sum, they constitute a speech community that is at the same time referring to already established rules of linguistic conduct and dynamically negotiating, contesting and changing them.

While a stable repertoire of communicative practices exists, for example in "typical expressions" (Bakhtin 2007: 87), rules of linguistic conduct, or frames, there is also a large "grey area" of contested denotations (Silverstein 2004), vagueness and ambiguity (Bhatia et al. 2003, 2005), and incomplete or non-existent formalization of language use. This provides scope for the emergence, through interaction, of rules of linguistic conduct, but also opportunities for partial "mutual intelligibility" in the communicative interaction within the IGC.

Much as in the speech community itself, the various communicative events in the committee are dynamic as to registers, norms of interpretation and instrumentalities. One might call this the emergence or nascency of a speech community, a state in which the undetermined or underdetermined communicative norms outweigh generic and structural conventions of interaction. This is, however, not only true for communicative and interactional patterns or rules, but also for the level of competence of participants. The emergent aspect is especially noteworthy considering the importance actors attribute to decisions of the committee, as they constitute a temporal, written, and thus linguistic, concretization of the dynamics of negotiation. It is safe to assume that all communicative events are to some extent always emergent and changing. But the bearing of dynamic aspects for the IGC and other international negotiations is special insofar that there are constantly new participants unaccustomed to its communicative specificities. They constitute an audience that can be critical of the statements being made during negotiations: it is, to some extent, a public sphere with changing norms of communication and interpretation. Transparency plays a big role in the making of this sphere. Both the possibility for observers to participate in meetings and the accurate documentation of negotiations makes it necessary for delegates to carefully weigh their statements.

This situation of transparency and of a dynamic speech community leaves actors with a degree of uncertainty regarding the rules that apply and to which they 
can be held accountable. Large parts of the communicative conventions of IGC negotiations are stable, and actors can rely on their validity. But the inclusion of new actors, the specificities of the issues under negotiation, as well as internal and external political pressures can cause changes in the way actors negotiate. Though (or especially because) the speech community and its attendant speech events are in an emerging and constantly changing state, an inattentive use of language can turn to one's disadvantage. As Don Brenneis notes, "it is critical to suggest that, while [...] social organization is seen as emerging in the course of talk, at another level it draws upon already shared notions of the structure of interactional practice" (Brenneis 1988: 228). Actors in the IGC are hence confronted with a "processoriented" and unstable component of communicative events within the speech community that can leave them in an insecure position as to the changing conventions that are relevant to that event. Yet, they nonetheless have to take into account already established patterns.

\section{Opening Statements}

The relationship between established communicative patterns and the dynamic aspect of communicative norms and conventions can be observed especially well at the beginning of an IGC session. Here, the agenda sets aside time for "opening statements". ${ }^{76}$ These are usually scheduled for the afternoon session of the first day of a committee meeting, after the session has been opened, the chair and vicechairs elected, the agenda adopted, and an ILC panel makes presentations during the morning. In the afternoon, once the report of the previous session has been adopted and certain organizations accredited, the opening statements begin.

Looking at the agenda, one might think that these opening statements are rather short, as the time allotted to this agenda item is rather brief. Yet, taking IGC 12 in early 2008 as an example, ${ }^{77}$ these statements can very well last until the middle of the third day of a committee meeting. Correspondingly, the IGC 12 report includes almost 28 single-spaced pages filled with delegations' opening statements. ${ }^{78}$ These are not full transcriptions of the recorded opening statements, but instead an attempt by the WIPO secretariat to render the gist of what was said in each statement contained. Thus, what appears in the report depends upon the actors of WIPO's secretariat compiling the report. Moreover, it has been IGC

\footnotetext{
${ }^{76}$ See WIPO/GRTKF/IC/14/INF/3 for the agenda of the IGC's $14^{\text {th }}$ session. Since IGC 17 in late 2010, opening statements have been discontinued as a separate agenda item. Nonetheless, the interplay between established patterns and dynamic aspects of statements as well as large parts of the specific contents of opening statements continue to exist in IGC negotiations.

${ }^{77}$ At subsequent and prior IGC meeting, the opening statements took a similar chunk of time.

${ }^{78}$ WIPO/GRTKF/IC/12/9.
} 
practice that after a statement is given, WIPO staff members walk through the aisles with a USB-stick in hand, collecting the electronic versions of statements from the speakers directly. Sometimes, speakers only have handwritten notes, and these are collected as well, copied and returned to the respective speaker. Some actors simply speak off the cuff, drawing on a habitualized communicative repertoire and using "boilerplate" sentences. Delegations can request amendments and changes to their statements as they appear in the report, so the final report can be considered definitive.

Yet, there are phrases and utterances that are usually - but not always - left out of the report. ${ }^{79}$ This practice differs from report to report, so that the report for IGC $16^{80}$ includes text expressing thankfulness or commendations for the work the WIPO secretariat has done, or utterances congratulating the chair and vicechairs for their election. The reports for IGC 12, 13 and 14 do not mention such matters, even though such statements were made and observed. Members of WIPO's staff, when interviewed, state that some parts of the opening statements are considered unimportant and hence left out of the report.

The interesting aspect here is to consider that reports that are generally viewed as "objective" in the sense that they reflect what was being said and refrain from interpretation are nonetheless influenced by who writes them (and, presumably, under what kind of circumstances). This might not have an influence on negotiations, and on a factual level it might very well be insubstantial, but it interferes with an ideology that stresses the importance of transparent documentation and its objectivity. ${ }^{81}$ After all, the linguistic phrases concerned are uttered over and over again in an IGC meeting, and so they presumably actualize a specific function or need.

Opening statements are, given their prevalence, an important communicative event in IGC negotiations. In conversational analysis, this genre would be called "greetings", involving delegation representatives from WIPO member states, NGOs, ILCs and other observers participating in the session. Naturally, there are also countless informal greetings between delegates on the first day of an IGC session or even before that in informational meetings and other diplomatic functions. It is telling who makes an effort to approach someone for a personal handshake and an

\footnotetext{
79 This is also mentioned in the preamble of the report of IGC 13: "This report summarizes the discussions and provides the essence of interventions, without reflecting all the observations made in detail nor necessarily following the chronological order of interventions" (WIPO/GRTKF/IC/13/ 11:4).

${ }^{80}$ See WIPO/GRTKF/IC/16/8.

${ }^{81}$ See Riles 1999. See Riles 2006 is for an excellent edited volume discussing documents from an anthropological and legal perspective. See Garsten and Lindh de Montoya 2008 for the notion of transparency in relation to transnational organizations.
} 
exchange of a few words during the leisurely approach to the formal session opening. ${ }^{82}$ Opening statements are not only made during the time the agenda sets apart for them. When a delegation takes the floor for the first time during a meeting, for the most part they feel the need to start their statement like an opening statement, even if the meeting is in an advanced stage. Much of their content and many of their aspects are reproduced during the whole session, so that the analysis of opening statements also sheds light on the broader communicative conduct during an IGC meeting.

Given that so much of an IGC meeting is spent with opening statements, it is worth looking at what is being said in these statements, and why delegations take so much time in making these statements. Furthermore, it should be inquired what actors compiling the official reports of IGC sessions, including the opening statements, deem to be important enough to be included in them. Do omitted utterances really have no importance for negotiations or for the purpose of a transparent documentation, and if so, why do delegations spend so much effort when part of what they say is considered insubstantial?

At IGC 12, a typical - but not rigid - sequence of tokens could be observed during these opening statements. ${ }^{83}$ First, the delegates from countries representing the regional groups were given the floor by the meeting's chairperson. They all started their opening statements with a sequence like the following:

1. Thank you Mr. Chair (sic!).

2. Country of Origin,

3. On behalf of Regional Group X,

4. Would like to congratulate you and your vice-chairs for your election;

5. Would also like to thank the former chair for his work;

6. Would like to thank the secretariat for its work

7. and for the preparation of documents.

8. Would like to express support for/appreciation of/commitment to/confidence in the IGC process;

9. Appreciates the work that has been done so far.

The exact sequence at the beginning of an opening statement varies, and not all these elements are mentioned in every statement. Yet, there is a general sequence reproduced to some degree by almost all member state delegations to the IGC.

\footnotetext{
82 The nature of the IGC as an emergent speech community as well as decision-making body is strongly shaped by the thickness of formal and informal communicative channels and the relations formed by them. Thus, examining these different channels, stages, and meta-stages (virtual communicative spaces that are not materialized in specific settings) can also facilitate an understanding of how actors are "enculturated" into the IGC context, at a substantive but also at a linguistic level.

83 As parts of opening statements are not reproduced in the official reports, these notes draw from observations made during IGC 12 and subsequent committee meetings.
} 
Even NGOs, ILCs, the indigenous caucus and other observers begin their interventions in this fashion, so there is an apparent convention with respect to beginning an opening statement. Some aspects of these opening statements are more prevalent than others and even expand to other communicative events. A "thank you" to the chair begins nearly every intervention or statement made during the main plenary session where the meeting's chair is present. Sometimes there are slight modifications depending on context, such as saying "Thank you, Mr. Chair for giving me the floor" or "Thank you, Mr. Chair, I am sorry to take the floor again" ${ }^{84}$ It is an acknowledgement of the chair's authority over the meeting and of the related status difference. A simple "Thank you, Mr. Chair" can thus also be understood as a contraction of a longer form that specifies why one thanks the chair. Combined with the other aspects found in opening statements, notably items (2) and (3), it is an automatic or habitualized way of affirming the framework of the meeting that one participates in. The conventionality to thank the chair stems from the sets of communicative norms of international negotiations and is not exclusive to WIPO's IGC. Its reason is described in WIPO's general rules of procedure $^{85}$ that apply to the IGC:

(1) No person may speak without having previously obtained the permission of the Chairman. (2) The Chairman shall call upon speakers in the order in which they signify their desire to speak. The Secretariat shall be responsible for drawing up a list of such speakers. (Rule 15: Right to Speak)

As the decision to give someone the floor lies with the chairman, it has become a convention for speakers to thank him for it. Such rules of procedure and corresponding communicative conventions can be found for other international bodies as well, and diplomats as well as all NGO and ILC representatives are well aware of them and reproduce them. Even in tense or critical moments in the main plenary negotiations where other norms of communicative conduct might be neglected, speakers almost never failed to address a "thank you" to the chair. This speaks for the habitualized character of such "inventory phrases" that are used over and over without having to reflect their specific meaning and function, as they are commonly understood and as there is a shared use of them. Despite the banality and "meaninglessness" of the phrase, it structures the communicative interactions significantly: speakers cannot address another delegation directly, because their intervention is always mediated by (1) the chair giving them the floor and (2) reacting to the chair for giving them the floor. A bilaterally directed exchange between two delegations thus requires an additional utterance that reestablishes the connection between speaker A and speaker B after the chair's mediation. To some extent, this

\footnotetext{
${ }^{84}$ These phrases have their equivalents in all the other languages used in WIPO negotiations.

${ }^{85}$ See http://wipo.int/freepublications/en/general/399/wipo_pub_399.html, accessed 9 June 2011.
} 
phrase and its conventionality hamper direct communicative exchanges between participants.

The phatic function of utterances in diplomatic negotiations similar to the IGC therefore has to be directional on several levels. This directionality is actualized by pivotal linguistic objects such as a "Thank you, Mr. Chair" or a subsequent reference to a specific delegation. They constitute one or more communicative channels that have functions on different levels, such as creating and affirming the communicative context, directing a question or demand at somebody, conveying messages to different audiences and so forth. As an initial marker, the "thank you" phrase frames the following exchange and creates a normative space with certain expectations, such as that the statement (and speaker) pays attention to rules and obligations of the speech community, apart from the chair's authority to regulate sequence, duration, and frequency of statements. Similarly, expressing who one speaks for or on whose behalf one speaks communicatively establishes the structural and institutional framework in which negotiations take place. ${ }^{86}$ The "representational complex", meaning the ways in which delegates are able to realize a representative function for nation-states and organizations, relies on such a "perlocutionary effect" (Austin 1962) of an utterance and its context. Name plates and name tags, or official lists of participants can have such effects directly, as they are based in what can be thought of as printed forms of utterances. Featuring in the structuring of the setting as well are personal acquaintance, habitual practices, the materiality of the whole setting including the building, its rooms and their decoration, security personnel and the procedure of having to officially register for an IGC meeting in order to be admitted to the building and main plenary room do all contribute to these functions, as do the shared knowledge and presuppositions about international organizations and negotiations among participants. Yet, the formal affirmation of the setting, its norms and communicative practices is in the

86 See Yankah 1995 for an ethnographic analysis of the function of "speaking for" someone in Ghana. Yankah paid special attention to the function of the "Okyeame" in royal Ashanti oratory. An Okyeame is a mediator for the communication between the Ashanti king, chiefs or priests and a second party. There may be as many as thirteen Okyeames, but usually there are several present. Their function is to find the contextually appropriate or fitting proverbs, metaphors or words to describe what the king or chief says. This presupposes both the competence to speak appropriately and knowledge of the specific social, cultural and political constellations of a given situation. An Okyeame is supposed to know more about these aspects than the king or chief he serves. Work by Chris Shore (2000) and others (Thedvall 2006) argues for a similar distinction in the EU: civil servants (or "Eurocrats") regularly surmise what the decisions of their superiors would be even in the absence of direct instructions. They thus at times also know more about the constellations of interests, motivations, goals, and power than their "chiefs". Initial inquiries at WIPO indicate this is partly true for negotiations the international level in at least some member states, yet "centralized" countries like France are said to consult back with the "capital" more often than others. Additional research on these issues in international negotiations is necessary to grasp their subtleties and contradictions that Aberbach, Putnam and Rockbach (1981) worked out for bureaucrats and politicians in Western Europe. 
end contingent on the open display of shared, sometimes even "ritualized" invocations that signal a common understanding of rules and structures that frame the discourse. Communicative conventions of IGC and WIPO negotiations are not formally fixed or documented. What is formally required is to signal one wishes to speak and then to wait for the session's chair to offer an invitation to make an intervention (see Fig. 6). Communicative conventions mainly stem from the interrelations of interactional practice in a specific field - the way things are done in IGC negotiations - and its historical dimension - the way things have been done in IGC negotiations.

The initial sequence of an opening statement is ritualized communicative behavior. The initial marker "Thank you, Mr. Chair" is so habitual that omitting it would be noted by IGC participants as something odd that requires attention. This gets very obvious when over the course of almost two whole days, delegation after delegation reiterates these tokens before segueing into more substantial parts of opening statements that differ from this sequence and include specific concerns, questions, and claims. At IGC 14 in June 2009, a representative of an indigenous organization in an informal setting jokingly proposed attaching two buttons to the doors of the main plenary room. The left button one would press if one would like to congratulate the chair and thank the secretariat, the right button one would press if one would not like to do that. By largely omitting these stock phrases from the official documentation, the WIPO secretariat signals, as do many IGC participants, that a "thank you" to the chair does not constitute progress in negotiations.

That may appear to deny the agenda item "opening statements" much weight in the IGC process, and certainly the initial formalities are of little consequence. But this genre goes beyond the scope of mere greeting formulas, both as it is not restricted to the contents of the initial sequence and as a contextualized analysis of the initial sequence shows that some phrases used by delegations in these initial sequences function as indexical tokens. What appears insubstantial is embedded in the opening of an IGC session, and foreshadows or signals positions actors will take subsequently. This argument is not meant to hypostatize the function of ritualized and marginally significant semantico-referential linguistic tokens, or see them as a functional necessity that establishes a discursive space that would otherwise be dysfunctional. The existence and persistence of opening statements is brought about by three other factors other than the conventions of formal international negotiations. The first is that of legitimation, as "habit" and traditions of structuring a meeting play a large role in evoking the sense that the proceedings are right and proper as studies from linguistic and legal anthropology have repeatedly 
shown. ${ }^{87}$ The second is that the progress of a given committee determines its meeting agendas. Relatively new international fora depend on opening statements to work out different perspectives and interests, while more seasoned committees are more "saturated" and do not need such drawn out coordinative measures. The third factor is that extending opening statements over several days also slows the process of reaching consensus or a decision Spending much time on opening statements, or with insubstantial interventions, is not always counterproductive for all participants. Even delegations interested in speeding up the negotiations can use the long opening statements to their advantage by utilizing them to frame subsequent negotiations in a broad manner that allows connections to other issues and fora to be made, which can be to their advantage.

The initial sequence of opening statements should not be reduced to a semantically and pragmatically irrelevant feature of IGC negotiations. It can rather be viewed as a phatic device constituting the social frame of the meeting. It functions as a communicative introduction of participants making the statements, their delegations and the regional groups they might speak on behalf of. It also functions to prefigure the position a given delegation will take and the alliances it may form. In this respect, the initial sequences of opening statements function as orientation both with regard to structure, meaning the communicative norms and conventions, and content, meaning the documents a delegation wishes to highlight and the prospects a delegation sees for the session.

The opening and closing of communicative events sets the tone for a given sequence. In Erving Goffman's terms, the greeting is a crucial component in establishing the stage on which the assembled actors will play (Goffman 1959). They are indicative of what is to be expected from subsequent interactions, and hence they are social "framing" devices (Goffman 1974) in the sense of "schemata of interpretation" that make it possible for participants "to locate, perceive, identify, and label" communicative events (1974: 21). ${ }^{88}$ Thus, greetings contribute to the way that things are understood and categorized as well as to the way that interactions are structured (e.g. turn-taking sequences). Ritualized linguistic tokens fulfill the task of framing interactions that are repeated over a given course of time and within spatial boundaries. Given the historical dimension of international negotiations within WIPO and other international fora, the communicative conventions have been actualized before their specific instantiation in a specific context, such as a session of the IGC. Moreover, aspects of communicative behavior specific to the IGC have been perpetuated within the IGC in its prior sessions, so that there is a

\footnotetext{
87 See Richards and Kuper 1971, Sherzer 1983, Brenneis and Myers 1984, Schwartzman 1989, and Richland 2008. All these studies acknowledge there is a constant negotiation between a given interactional structure and the process of interaction that actualizes the specific conventions. The same, I argue, holds true for WIPO's IGC.

88 Note the similarities between Goffman's frame analysis and Myers-Scotton's markedness model (1998) concerning the role of expectations and obligations in a given communicative event.
} 
generalized understanding of how negotiations work as well as a shared set of expectations of what communicative rules participants are going to adhere to. A "new" negotiation of communicative conduct is thus not necessary because of this structural and interactional implicitness that reproduces schemata of interpretation and patterns of interaction. In this respect, the initial sequence of opening statements contributes to the reproduction of interactional frames, to the acknowledgment of already given interactional patterns and to the acknowledgement of the speaker's participatory status within the committee: the structuring of opening statements with its implicit assumptions about hierarchical (the chair's authority) and interactional features of the committee as well as the speaker's subscription to these conventions and his or her perception that other participants share these conventions - all these aspects are part of the initial sequence of opening statements.

Roman Jakobson has argued that the functional hierarchy of greetings places the phatic function first while other functions - referential, emotive, conative, metalingual, and poetic - are activated to a lesser degree (Jakobson 1960: 356). This supposition, that greetings are primarily "empty formulas" might explain the ease with which certain utterances are left out of the official report, while others are regarded as more important.

The two facets of greetings as - for the speech community - functionally efficient and semantically irrelevant communicative events do not contradict one another. A communicative event such as a greeting can frame an interaction while primarily actualizing a phatic function, and subordinate other functions. Michael Silverstein points to the existence of "shifters" as "pure indexical tokens" or "nonreferential pragmatic tokens that serve as social indexes and make the social parameters of speaker and hearer explicit" (Silverstein 1976: 34). They have little to no semantico-referential meaning and at the same time realize a pragmatic function. A "Thank you, Mr. Chair" following this would be a deference index that points to the difference in power between the chair and a given participant, the social difference being that the chair has the authority to invite a delegation to speak, while the delegation speaking has only a proxy authority bestowed upon it by a national or regional body, or some other organized institution. As a courtesy token or honorific, the social dimension of the utterance is of greater importance than its semantico-referential content. Viewing the initial sequence from this perspective makes clear that the utterance depends on its context and that an analysis of this utterance needs to take its broader context into account. The initial sequences of opening statements are thus indexical tokens that implicitly and explicitly validate both the work of the committee and the rules and conventions of speaking established in the IGC, while differentiating social roles within the committee and its shared communicative activity.

Yet, there is more to opening statements than this initial sequence. It is telling that the Peruvian delegate at IGC 13 made an explicit distinction between the for- 
mal part and the substantive aspects of his opening statement, ${ }^{89}$ proceeding, after the initial sequence, on to note a proposal by the African Group that suggested implementing inter-sessional expert working groups. The report of IGC 13 does not mention the distinction he made. The formal aspects are reproduced in the report as follows:

The Delegation of Peru noted that it was not a coincidence that the Committee was the first meeting that the Director General was participating in as Director General. Indeed, he had contributed greatly to the work of the Committee, he was committed to its work and the work that was ahead and, as he had clearly said when he took up his functions as Director General, the need to have a specific outcome after so many years working on this matter in the Committee. The work the Secretariat had carried out in producing the gap analyses documents was fairly positive. These documents would enable the Committee to find common ground and be able to make progress in the work. ${ }^{90}$

After these aspects that fit the initial sequence, he continued with more specific comments with regard to substantive and procedural issues as well as the mode of work of the committee:

[...] Peru supported the holding of inter-sessional meetings as long as they were geared towards speeding up the work in accordance with the mandate of the Committee. [...] The African proposal, as far as Peru understood it, sought to focus the work of the Committee through the creation of a group of experts, which would meet between the sessions. The proposal was, in principle, a positive proposal, which in all likelihood would have to be refined, but was an important proposal to make progress between the sessions and to achieve a final and concrete result before the General Assembly of next year when the mandate of the Committee ended. (ibid.)

Thus, after a thank you to the chairman for being given the floor, a congratulatory statement to the chairman to his election, and a (positive) assessment of the work done by the committee so far, opening statements are used by participants of the IGC to furthermore initially frame their intentions and strategies for the subsequent meeting. While the structural elements of these opening interventions appear to be nearly identical, their verbal content and their performative contours express a great deal about the level of engagement and approval as well as the in-

\footnotetext{
${ }^{89}$ Author's note from IGC 13.

${ }^{90} \mathrm{WIPO} / \mathrm{GRTKF} / \mathrm{IC} / 13 / 11: 8$.
} 
tent (or lack thereof) to work by a particular delegation in a given session. There are numerous delegations who simply use the "thank you" and "congratulations" part of the greeting, in some cases because a mere spectator role is intended.

\section{Language Politics in International Negotiations}

Some delegations, however, use the opening comments to signal goals beyond conducting a courteous debate. Thus the Chinese delegate's opening statement in December 2006, in Chinese and simultaneously translated, began with a profuse thank you followed by a request to broaden the language base of the committee. English, she said, was not anyone's native language in her delegation, hence working through the written papers in preparation for the meeting was very timeconsuming. In order to be able to fully participate, preparatory papers in Chinese were requested. Such requests for additional translation of working documents are numerous in the IGC, but also in other international fora where English-based documentation and negotiations are standard Yet, many delegations do not have the competence to express their views and to realize their pragmatic strategies adequately and in a manner also conducive to their interests. During the IGC sessions observed, the main claims were for Arabic, Chinese, and Russian to be considered as working languages. ${ }^{91}$ For some IGC participants, issues of translation and interpretation play a large role in negotiations and can have implications for how well a delegation's perspective can be communicated. Moreover, the contingencies and ambiguities in texts are multiplied if the negotiated text is available in many languages simultaneously. One might argue that this is also a reason for the relationship between nouns and verbs in international negotiation texts, where nouns are relatively stable as they are repeatedly used and take on the form of "typical expressions" (Bakhtin 2007) in various languages, whereas the negotiation of meaning and implications of texts largely centers around verbs and their specific grammatical modes (subjunctive, imperative, etc.). Of course, this question concerns pragmatic strategies as well, as these depend on a shared understanding or a shared negotiation about the consequences of communicative action.

Issues of language use are illustrated in what follows by several cases, though the last is of somewhat lesser importance in the context of IGC negotiations.

(1) The first case asks whether an interpreted statement by a delegation really does reflect what that delegation wants to express. Several delegations, but most

\footnotetext{
${ }^{91}$ The issues of translation and interpretation within the IGC are manifold. A B.A. thesis on the role of interpreters in IGC negotiations has been written by Verena Pohl, a research assistant of the DFG Research Unit on Cultural Property; she covers some aspects of this issue.
} 


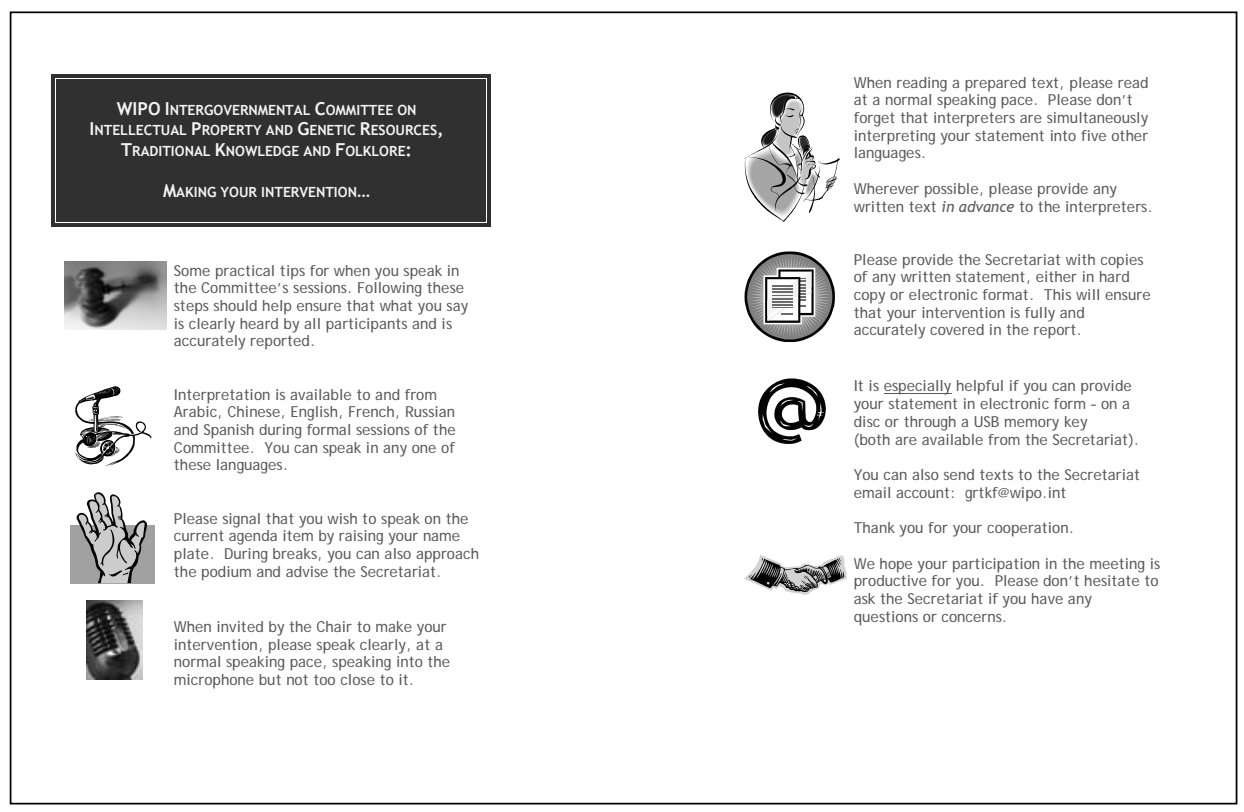

Figure 6. Leaflet distributed at WIPO's IGC 12 in February 2008.

notably China, have developed the habit of having one member of the delegation read out a statement sentence by sentence in Chinese, while a second delegate listens to what is being translated. The speaker only proceeds if he is given a signal that the translation is accurate. Another way to try to ensure accuracy is to hand in a written version prior to making the verbal statement, so that the interpreters have more than simply verbal utterances to translate and are able to translate a statement more accurately. Fig. 6 shows a leaflet that encourages delegates to follow this practice, along with the suggestion to read out statements slowly. In a number of cases, statements were read out too fast so that the interpreter had to stop the speaker. This almost always had the effect that the content of the statement was not understood by delegates dependent on the translation.

Related to this is the question of legal certainty of translated statements or documents that are not available in a specific language. The main concern here is that by translating quasi-legal negotiation text, the intended meaning may change in the target language and the text can have unintended implications. Similarly, text only available in English poses the difficulty of translating terms and concepts to other languages and legal frameworks, creating uncertainty for negotiations. At IGC 15, reflecting these issues the delegation from Spain stated:

[01] The Delegation of Spain referred to Circular 7767 prepared by the Secretariat, concerning the translation of Member States' contributions into different languages. It added that this was contrary, ipso 
jure, to the standards and practices of the United Nations. It stated that it was not able to consent to any potentially-binding document which had not been translated into Spanish and distributed with sufficient time for its analysis. ${ }^{92}$

After a clarification by WIPO's Director-General "that the Secretariat would do its best to make them available in the official working languages, but with no guarantee" (7), this statement was endorsed by the delegations of Portugal and Algeria. To give their argument more weight, the Portuguese delegate, in particular, referred to the broader issues of language and highlighted that "the languages and cultural diversity were very important values in the United Nations system" (ibid.).

(2) The second case concerns misunderstandings due to translations. A vivid example is a joke made by a delegate from Peru that referred to "la maca", an Andean plant said to have aphrodisiacal properties. Spanish-speaking delegates understood the joke and its innuendo and laughed accordingly. Yet, the interpreter translated "la maca" as "la hamaca" - "hammock" - whose short colloquial form is also "la maca". Those listening to the English translation were left baffled. This translation error was not corrected until the Peruvian delegate was approached by another delegate after the session and asked to clarify the mistake. The number of such errors is high, especially in the context of jokes, proverbs, or sayings (such as the Latin American colloquialism "¿De qué color es el caballo blanco?" that was translated in one IGC session as "What color is the green horse?"), though the implications of such errors are hard to assess. One can assume that in relation to more typical phrases there are less errors as those are also habitualized to some extent by interpreters, but such cases have still to be considered.

(3) The third case relates to the immediate effects of speech and its presentation. Statements can be read out very monotonously and soberly, which is often the case in interventions in IGC negotiations. Yet, there are also delegates from WIPO member states, NGOs, IGOs and ILCs and even the meeting's chair that act differently. In numerous instances, statements were performed passionately, furiously, desperately, sarcastically, ironically, metaphorically and jokingly, sometimes following drafts and sometimes enacted without notes. These performances and their subtleties require considerable competence on the part of IGC participants to decipher, even without translation. Delegates who are generally competent in English might not get all the nuances. Much may be lost when such emotive statements are interpreted, and it largely depends on who is interpreting.

There are substantial differences between the interpreters. Some of them unseen from the plenary room - "reenact" emotional statements, even with gestures and a similar intonation. Others take a more sober approach and mainly reproduce the contents of a given statement without paying much attention to into-

\footnotetext{
${ }^{92}$ WIPO/GRTKF/IC/15/7:6.
} 
nation, emphasis or rhetorical devices. This can also be due to the technical and natural problems of interpretation, including volume or tempo. Some delegations are well aware and self-reflexive about the difficulties of translation and interpretation.

At IGC 14, a Swiss delegate chose to make a statement in Spanish rather than in French or English. In a conversation after his statement, he argued that the hegemony of English in this setting was disadvantageous for non-native English speakers, and that the large number of Spanish-speaking delegates called for strengthening language diversity. Untranslated statements in the IGC and other international fora were better received, he argued, and a speaker could have a much larger impact on his audience in terms of attention, comprehension, and empathy if he spoke in their native tongue rather than making them rely on a translation. Similarly, a multilingual Peruvian delegate at IGC 14 chose to make several statements in English rather than in Spanish. Several sentences into his first statement, he switched to English, making a reference to the Swiss delegate who spoke Spanish, whereupon many IGC participants paid noticeably more attention to what he said. He argued in a private conversation afterwards that it was much easier to gain the attention of the audience when speaking in their respective mother tongues, and that many substantive as well as rhetorical aspects were lost in translation. ${ }^{3}$ The implications and problems of interpretation and translation are an important issue in IGC negotiations that expands from oral to written statements as well as to the drafting of text.

The performative aspect of speech ${ }^{94}$ in negotiations mainly depends on written decisions and has considerable influence on them. It creates powerful dynamics that can change the course and outcome of a meeting. The creation of such dynamics is to a large part also contingent on questions of translation and (foreign) language competence, which - together with other factors - might help to explain the reflexivity about them as well as calls for the inclusion of additional working languages.

\footnotetext{
93 The representative of the indigenous group Tupaj Amaru concluded his opening statement (one interrupted by the chair calling him to order) with the words: "I could speak French or Russian but I notice that there will still be no progress" (author's field-notes from IGC 13). This shows that such language issues are of importance, but yet they should not be overestimated. National and commercial interests as well as strategies play a much more important role in WIPO negotiations than the language used.

${ }^{94}$ See Richard Bauman "Verbal Art as Performance" (Bauman 1977) for an analysis of the performative aspects of speech; Briggs 1988 for a reflection on the interrelation between competence and performance in Mexicano verbal art; Bauman and Briggs 1990 for an overview of studies of poetics and performance from the perspective of linguistic anthropology and folkloristics; and Kapchan1995 for an article on performance from the perspective of folkloristics. The ethnography of speaking has adopted many of these methodological and theoretical considerations. In its early stages, some aspects of verbal performance were taken into account in Hymes' SPEAKING model (Hymes 1986), mainly as part of the factors act sequence, keys, instrumentalities, and genre.
} 
(4) Finally, the fourth case is about the translatability of concepts in intercultural settings. ${ }^{95}$ In WIPO's IGC, concepts like tradition, property and ownership, community or law are central. ${ }^{96}$ These might be understood as universal concepts ${ }^{97}$ with equivalents in each specific society or community. Some WIPO staff members argue this is the case. ${ }^{98}$ Yet, one can also argue that some concepts, such as intellectual property, knowledge or individual creativity, stem specifically from the tradition of the European Enlightenment and cannot be simply applied to other cultures. ${ }^{99}$ This second view has been argued by folklorists and anthropologists for some time. They question the assumed universality of central categories in the intellectual property system, mainly of individual creativity, possessive individualism, the incentive structure of property rights, and the authenticity of cultural creations. ${ }^{100}$ The gist of these discussions is that sometimes there is no "structural fit" between the categories used in international fora and the local realities (CIEL 2008, Shand 2002); that complex adaptation measures may be needed to mediate between different levels (Pfaff-Czarnecka 2005); and that such processes can have negative and unintended consequences for the social groups involved (Noyes 2003, Tauschek 2010).

Three of many examples from the IGC are especially noteworthy in this regard. The first refers to two different meanings of the word "tradition" in Arabic.

\footnotetext{
${ }^{95}$ See Sarat Maharaj's work on difference and untranslatability (Hall and Maharaj 2001) for a discussion.

96 The concepts of heritage play a role here, too, as the issues negotiated in the IGC overlap with those discussed in the various UNESCO fora and conventions on World Heritage and tangible or intangible culture. See Bendix 2009b for an examination of the semantics of heritage and inheriting that addresses the implications of different semantic approaches to these issues.
}

${ }^{97}$ See Marceau 2007 on the question of universality of collecting cultural artefacts. This discussion ties into the larger anthropological debates on cultural relativism (Spiro 1986 for an overview, Dundes-Renteln 1988 for the connection to human rights, Geertz 1984, Brown 2008) and linguistic relativism (Duranti 1999c for an overview, Kay and Kempton 1984, Martin 1986). Put simply, the relevance to the IGC are the questions whether there can be, are, or should be shared norms, practices, and understandings across different "cultures", meaning in relation to concepts and institutions of property or tradition.

${ }^{98}$ Interview with a member of the IGC secretariat, October 2008.

99 See Nadasdy 2003 for different conceptualizations of "knowledge" in First Nation contexts that conflict with the notion of knowledge as used in national bureaucracies; Yarrow 2008 for a case study from Ghana; and Strang 2006 on the differences between anthropological and indigenous knowledge. See also Raven 2006 for an article on "protocols" as a way to regulate relations between bureaucracies and indigenous communities.

100 See especially Hafstein 2004, 2008, 2009, Rikoon 2004, Kasten 2004, Hann 1998, and Kuutma 2009a, 2009b for the debate on the relation between culture and ownership in international and national settings; Noyes 2006, 2007 for an examination of the connections between collective creativity, ownership and cultural property processes; and Bendix 1997 for a critique of the notion of authenticity. 
One meaning connotes a rather static and fixed form, the other implies it is dynamic and evolves:

[T] here are sometimes errors ... that creep in - difficult terms like "tradition", "folklore", "culture". Sometimes these difficult terms we've had ... complaints. ... Not very often but now and again we've had a delegation saying that it wasn't translated perfectly correctly. There's one issue that is interesting, and that is the word "tradition" in Arabic. ... I've heard several times ... the Arabic, the Egyptian delegation complaining [about how] ... we use "traditional" or "tradition". In Arabic, there's more than one word that one can use for that. One of those Arabic words emphasizes "tradition" as being old, something of the past, and another word emphasizes that it's also something that's renewable, something that can also be new. And if I remember correctly, the delegate from Egypt prefers the second version of the word and our [translation] unit insists upon using the first meaning. Now this is all subject to correction. I don't speak Arabic and I don't know what the discussion's about, but that's a good example ... of whether there is a real linguistic difference which has a substantial bearing. ${ }^{101}$

The question then was to figure out which one of the two terms was (more) in accordance with the term "tradition" as used in IGC negotiations, and which one could be translated to be in compliance with the underlying concept.

The second example concerns the translation of WIPO documents into kweyol, a version of Antillean Creole spoken in Saint Lucia. The sociolinguist involved argued that many concepts used in negotiations and IGC texts had no counterpart in kweyol, and it was thus necessary to translate the meaning of concepts such as individual creativity or knowledge in order to facilitate an understanding of key issues for local communities. ${ }^{102}$

The third example is the frequent claim by ILCs and other IGC participants that culture or tradition cannot be viewed as a separate sphere of social life. Rather, they argue that a holistic view is necessary to understand the complex relationships between people, their land, their knowledge, and their tradition. The separation of TK from TCEs, for example, would thus inevitably violate the claimed cultural holism. Thus it would be extremely difficult to translate this ho-

\footnotetext{
${ }^{101}$ Interview with a member of the IGC secretariat, October 2008.

102 Conversation with the representative of the Bethechilokono of Saint Lucia Governing Council, BGC, at IGC 12, March 2008. The BGC is only one of several ILCs and NGOs that works towards facilitating the exchange of information, in particular to educate local communities and groups about the work of the IGC. WIPO also undertakes "capacity-building" programmes with the same intention.
} 
lism into the concepts used in the IGC process or other arenas dealing with cultural property. ${ }^{103}$

While there are such examples for the difficulties of conceptual translations between different languages and social contexts, I would argue that they are only marginally relevant for the IGC negotiations. First and foremost, this is because almost all IGC delegates have some prior experience and are educated professionals with competence in intellectual property matters. It can be assumed they all grasp the gist of core concepts relevant for the IGC, and can evaluate specific commonalities and differences. Added to this, the documentation is so dense that finer points concerning the understanding of concepts are explained and are furthermore explicated numerous times during the meetings, such as during sessions of the indigenous panel. The issue of translatability of concepts is thus largely negligible.

Still, there are of course ideological, substantive, and technical differences in the use of concepts and associated terms. The questions about the commensurability of concepts and ideas cannot be solved through the IGC, and a legal instrument cannot fully take these different conceptualizations into account. Instead, differences can be viewed as differentiating perspectives on specific aspects rather than as fundamental, unresolvable distinctions that hinder shared understanding. There is enough knowledge, competence, and willingness to understand differing positions with respect, for example to cultural holism on the one hand and the concept of the individual creator on the other. Whether such differing perspectives are recognized or included in decisions is something else, but it does not depend on a lack of basic understanding or an untranslatability of concepts.

Assertions about language politics are an aspect of opening statements that expand into "substantial" questions raised in the ensuing negotiations. Referring to such issues of language is a way a delegation's interest in having a specific language be revalued can be fostered, and opening statements are a way to voice such claims in a formal and documented way. Language politics are moreover not confined to the IGC, but need to be analyzed in the larger context of the UN system. Efforts to make working documents available in Chinese or Arabic must also be understood as attempts to strengthen the position of national or regional groups within the entire UN system. Repeated references to claims for the inclusion of a specific language in various fora increases the pressure on other delegations to consent to them, and such pressures can be transformed and added to other arguments or disputes as bargaining tokens. ${ }^{104}$

\footnotetext{
${ }^{103}$ See Blakeney 1999, Marinova and Raven 2006, Graber 2007.

104 See Schiffman 1996, Mar-Molinero 2000, Castiglione and Longman 2007, Nic Craith 2007 and Kamusella 2009 on language politics that are relevant for this question; see Gad 2006 for a discussion of representational fairness in WTO rule-making that also addresses some language-related issues of negotiations. The body of literature on language ideology, mainly from linguistic anthropology, is also worth noting. See Errington 2001 or Kroskrity 2004 for introductory papers on this issue.
} 


\section{Opening Statements as Framing Devices}

One can examine a number of exemplary opening statements in more detail and as framing devices in order to distill other substantive aspects from them. ${ }^{105}$ Language politics are only one aspect that is referred to in such statements, and there are other patterns that can be found that have a bigger influence on the specific negotiations. The statements chosen here serve as examples and as a synopsis of the main aspects of opening statements.

At IGC 12 in early 2008, the agenda item "opening statements" took up nearly two full days of session, thus had more than marginal substantive value. Taking the statement by the delegation of Algeria on behalf of the African Group - the first opening statement made during IGC 12 - as an example, one can recognize a number of specific aspects:

[02] The Delegation of Algeria on behalf of the African Group reiterated the importance it attached to the work of the Committee and the issue of genetic resources (GR), traditional knowledge (TK), and expressions of folklore (EoF), and its desire to contribute in a positive and constructive fashion to the negotiation process which was under way. TK and GR had played and still played a vital role in the day to day life of the bearers and beneficiaries of such rights or knowledge. The interest in their protection rested not only in their attachment to the traditional cultural and scientific heritage but also the benefits arising from this TK as a source of well-being and cultural scientific and economic development. Indeed they were key to food security and key to the health of millions of people in the developing world. In many developing countries a great part of the population depended on traditional medicine. Modern medicine being inaccessible, traditional medicine remained the only way for affordable care for the less well-off. Yet the traditional methods and medicinal plants were tested and exploited without the knowledge and consent of indigenous communities by third parties to develop medicines and other products which then themselves benefit from protection. ${ }^{106}$

Beginning with a rather general appreciation of the negotiation process (items eight and nine in the initial sequence, noted above), the statement goes on to note the relevance and urgency of issues related to GRTKF. Individuals and groups

\footnotetext{
105 The statements analyzed hereafter are largely reproduced from the official meeting reports produced by the WIPO secretariat on the basis of the written statements collected by WIPO staff during the session. They are thus not verbatim but - apart from parts of the initial sequence - nonetheless reflect quite accurately what was said by delegations. They are complemented by fieldnotes by the author.
}

${ }^{106}$ WIPO/GRTKF/IC/12/9:9-10. 
affected by the lack of protection of TK and GR - "bearers and beneficiaries"107 are particularly emphasized, and the statement elaborates on issues of food security and health. There is also the assessment that due to "piracy"108 the situation is getting worse:

[03] At the same time as this meeting, TK, GR and TCEs of entire peoples were being pirated and the rights of local communities were being infringed. This led to a serious situation which was getting worse without any definitive and acceptable solution for all being found, despite the efforts deployed over the last two decades.

Framing the issues as not only synchronous but also causally connected to poverty, food shortages, and misappropriation creates a reference point outside the IGC, that can nonetheless be influenced by the course of the subsequent meeting, thus from within the IGC. This reference simultaneously locates the issues being negotiated as taking place outside the IGC, in the specific local contexts and processes, as well as inside the IGC, which makes them less abstract at both levels.

The statement refers not only to the general issue of the misappropriation and lack of protection for GRTKF but also to the potential direct influence IGC participants can have. The moral dimension created here is - even if it is mainly a discursive construction - strategically advantageous for the claims of the African Group. It postulates an underlying imperative or guideline differentiating between a good course of action, namely acting against the misappropriation of GRTKF, the bad course of action implied by doing nothing. The statement introduces several differing actor categories: (1) "entire peoples" and "local communities" as "bearers and beneficiaries" of TK and TCEs. Their rights are being infringed upon, and they suffer from poverty, bad health care, and food insecurity; (2) actors who speak on behalf of them in a representative capacity, such as the African Group and its member states ${ }^{109}$ or organizational actors such as OAPI and ARIPO

\footnotetext{
107 The terms by which "holders" of TK and TCEs are named play an important role in negotiations as well as in the drafting of specific legal text. Among them are "holders", "bearers", "guardians", and - as a more technical term that denotes a group of actors within an access and benefit sharing (ABS) system - "beneficiaries".

108 The notion of piracy in the context of the IGC mainly stems from discussions in the realm of GR, bioprospecting and biopiracy; see Peterson 2001, Hayden 2003a, 2005 for an anthropological discussion, and Mgbeoji 2006 for a legal perspective with a specific focus on indigenous communities and bioprospecting. In the context of WIPO, the term "piracy" is mainly used by ILCs and NGOs while the term "bioprospecting" is rather uncommon, perhaps because genetic resource issues are addressed less than issues of TK and TCEs in the IGC.

109 The statement by Algeria includes the phrase "most indigenous local and traditional communities of the Member States", which hints at the conceptualization of the nation-state as the guardian of both ILCs and GRTKF. This view of indigenous communities as subordinate and non-autonomous entities is frequently held by African countries and other member states.
} 
that adopt legal instruments to actively protect the TK and TCEs of actors in category (1); (3) "third parties", meaning "research institutions and companies" which actively misappropriate or "pirate" the traditional knowledge of ILCs and infringe their rights; and (4) the implicit category of actors that neglect or do not understand the urgency of the issues and hinder substantial progress in the IGC.

This categorization is similar to the "membership categorization devices" described by Sacks. The best-known example is the sentence "The baby cried, the mommy picked it up" (Sacks 1972a), where the relationship between the two actors referred to in the sentence is inferred by contextual social knowledge. Sacks argues that due to social categories assumed by the recipient, the mommy in this example is automatically understood to be the mommy of the baby, with membership categories grouped as "standard relational pairs" that constitute the frame for the interaction and its interpretation. These include rules and obligations of the communicative exchange, here the mother's obligation and right to attend to the needs of the baby.

In the case of the IGC, the Algerian categorization creates an opposition between groups (1) and (2) and groups (3) and (4). It positions the speaker on the morally "good" side of the two sides and vests authority in the speaker to perform a representational function for this "good" side. For the other side, meaning group (4), which in the IGC context largely means the Group B countries, an opportunity is opened to change this situation as it provides two possible ways to frame a position. On the one hand, they can distance themselves from the implicit reproach by supporting ILCs and thus the African group. On the other hand, if they fail to do so, they risk being accused of directly supporting the misappropriation and piracy of TK and TCEs. However, this setting up of opposites is only partial. Further differentiation of actor categories is possible, and the diplomatic setting itself does not allow for too direct accusations. Nevertheless, it creates a referential space in which differentiated positions and perspectives have to be mediated between the polar opposition of "good" and "bad" categories of actors, even if they are only constructions.

The Algerian delegate accordingly continues:

[04] The African Group encouraged the Committee to speed up its work in order to find specific and tangible results that would meet the expectations of most indigenous local and traditional communities of the Member States. They hoped the process under way would lead in a speedy fashion to the adoption of a legally binding international instrument to counter the misappropriation and the misuse of TK committed every day to the detriment of their heritage.

Again, this passage illustrates a differentiation between groups of actors, partly as participants of the IGC, and partly as outsiders. Particular local actors - "indige- 
nous local and traditional communities of the Member States" - are referred to in conjunction with the African Group, which is portrayed as representing their interests in the committee. On the other hand, acts of misappropriation and misuse are mentioned that require an immediate response by the committee, as they are committed on a daily basis. This construction of urgency claims complicity in misuse and "piracy" on the part of actors who could do something about it but choose not to. The plea to draft and adopt a legally binding international agreement for the protection of GRTKF is complemented by the construction of a dichotomy of actors, indicating possible ways a delegation can position itself in the negotiations. In this, the speaker's position is portrayed as the only viable or "good" way to act. As concerns time, the situation ILCs are facing is portrayed as urgent, and thus the IGC's actions are constructed as urgently necessary as well. Two different but interconnected spheres are framed in a specific "temporal mode" suggesting the subsequent session should prioritize concrete results over drawn-out discussions.

The statement continues on, outlining possible scenarios for legal regimes and mechanisms at the international level, and drawing on specific national experiences in GRTKF protection. The form is that of an authoritative discourse as to substantive questions and specificities, which not only evaluates the situation, but projects the image of a speaker who is objectively capable of assessing the various legal instruments available and their implications. This signals that outstanding problems have already been solved by a competent authority (the speaker) and that there is already a factual basis for answering specific questions in a brief amount of time. The Algerian delegate states:

[05] Indeed, the African instruments proposed constructive solutions to questions that continue to be controversial within the committee. Among these were the issues of the beneficiaries of such protection, as well as the objectives of protection, the exceptions or limitations to the rights linked to cultural expressions and TK which are eligible for protection and the duration of the protection that they are to be granted. The difficulties met in the area of a definition of certain concepts should not serve as an alibi or a subterfuge to delay further this process globally. The contributions made by Member States on the basis of the twenty issues previously identified and the discussions on the questions of substance debated in the Committee's recent sessions should enable progress on the substantive issues.

According to this way of framing the issues, the onus of proof that there is an alternative solution lies with the opposing parties in the negotiations. As the matter is of urgency in local contexts, and as the implicit question of morally "good" and "bad" actors is answered, and as the factual base on which the rest of negotiations can take place has already been established, the burden to follow suit and act is 
placed on the "other" side of the dualism of actors. Preemptively, the statement declares that questions of "definition of certain concepts" are, due to the already established factual base, merely an alibi and do not constitute a real problem.

Framing IGC issues like this has both advantages and disadvantages. Moral aspects can be strategically harnessed to bolster arguments of the African Group. Even if they are contradictory and inconsistent with policies on the national levele.g. regarding the legal status of indigenous groups -, it is hard to argue against them in diplomatic settings. They require an reaction by opposing delegations, and this reaction cannot simply rebut the claim, but needs to reframe the issue to argue against it. However, such a strategy can also be problematic as it creates a situation of conflict potentially hindering consensus.

Taking the statement by Slovenia (on behalf of the European Union) during this IGC as a second example, one can see how this "other" side reacts. The statement starts with the common initial sequence and a general expression of appreciation for the work and progress of the committee since its creation. It continues:

[06] The EU continued to believe that one of the most important achievements of the Committee had been to recognize the importance of TK, TCEs and GR to traditional and indigenous cultures worldwide. [...] It continued to support the Committee's spirit of open and responsible collaboration, and looked forward to further progress in the form of consensus solutions. However, the EU also recognized the inherent difficulty that had been encountered by the Committee in defining the essence of TK and TCEs, and the methods that could be used in protecting them. It was not enough to create a definition of what was obviously TK or TCE. The line between what was TK or TCE, and what was not, had to be identified. The European Community and its Member States recognized that some Members of the Committee might wish to reach a practical conclusion of its work within a certain time, for example, during the current mandate of the Committee for the biennium 2008 to 2009. Such a timely result for the work could be achieved in areas where a consensus had already been reached or nearly reached and only if flexible solutions were considered that would not bind individual countries to commitments that did not necessarily meet their needs. With respect to the schedule of the meeting, the EU insisted that sufficient time should be reserved for discussing GR as the last item on the agenda. The Committee now had a great deal of information before it. Therefore, the Delegation encouraged the Committee to focus primarily on the areas where consensus was possible in the short term, 
in order to achieve a practical result that could be accepted by all Members. ${ }^{110}$

This intervention was made immediately after the statement by Algeria on behalf of the African Group. For this reason it cannot be viewed as an immediate reaction to the first statement. Rather, the specific aspects that make reference to the points taken up in the prior statement can be understood as having been drafted in anticipation of the position the African Group would take. Similarly, the African Group statement anticipated the issues of definition taken up in the statement by the EU. The two opening statements thus hint at the history of the IGC and delegates' knowledge thereof, thus allowing for an evaluation of how delegations will behave during subsequent meetings. Both prior meetings and documents preform the content and structure of an IGC session, as do inter-sessional communication processes between regional groups, member states, individual delegates based in Geneva and the WIPO secretariat. ${ }^{111}$ Furthermore, the aforementioned habitualized aspects - stock phrases, formulations, and the modality of arguments - of speech play a role in the way that statements are drafted in anticipation of a specific audience. This aspect is of much importance for the analysis of IGC negotiations, and is part of what constitutes the IGC as a speech community.

Slovenia's statement on behalf of the EU begins with a very abstract and short reference to the subject matter:

[07] to recognize the importance of TK, TCEs and GR to traditional and indigenous cultures worldwide.

In contrast to the statement by the African Group, no specific actors or issues are mentioned. While the Algerian delegate talks about very specific problems - the misuse and misappropriation of GRTKF, food security, health, poverty - in a graphic way that includes groups of social actors that suffer from the current situation, analogous references are missing from the European statement. Instead, it focusses on "recognition", in the context of international negotiations a very weak expression that does not include proposing any specific action. The specificities of this recognition are neither explained nor are reasons given why one should recognize (such as the misuse the African Group alludes to). "To recognize", in contrast

\footnotetext{
${ }^{110}$ WIPO/GRTKF/IC/12/9:10-11.

111 In the case of the question of definitions, documents WIPO/GRTKF/IC/9/4 and WIPO/ GRTKF/IC/9/5 on "revised objectives and principles" for the protection of TK and TCEs foreshadow the development, as do documents from IGC 11 in July 2008 where comments on these two documents are collated that partly address the problem of defining TK and TCEs as well. Specifically the revised objectives and principles and the lists of issues (WIPO/GRTKF/IC/11/4(A), WIPO/ GRTKF/IC/11/4(B) and addenda for TCEs, and WIPO/GRTKF/IC/11/5(A), WIPO/GRTKF/ $\mathrm{IC} / 11 / 5(\mathrm{~B})$ and addenda for $\mathrm{TK})$ have to be mentioned here.
} 
with "to counter" (see [04]), is a weak and non-materialized process with a vague content and vague implications.

One could argue here with Austin (1962) that distinguishing whether an utterance is locutionary or illocutionary is difficult in the context of diplomatic negotiations. when it contains as a verb "to recognize". The aspect of "doing" something material, in the contextual sense of the verb "to recognize", is liminal and mainly defined by its negation, meaning by the absence of any particular actions apart from the ideational level. Furthermore, it is a very common phrasal expression in legal texts as well as international conventions and treaties, ${ }^{112}$ and one usually used in preambles to express the ideational foundations of a normative text, but lacking any legally binding force. ${ }^{113}$ "Recognizing" has a soft and flexible quality, and a lack of specificity to its associated actions. Coupled with the object of recognition (the "importance of TK, TCEs and GR to traditional and indigenous cultures worldwide"), there is no reference either to a specific set of situations or to specific groups of actors.

While the statement by Algeria mentions social inequalities and injustices that call for immediate action, the EU statement does not. ${ }^{114}$ Moreover, the Slovenian statement claims that recognition has already been achieved by the committee, meaning no element in the EU's intervention points to further goals to be achieved by the IGC. There is an allusion to the protection of TK and TCEs (see [06]), but it is defined ex negativo by the doubt expressed that the methods needed can be consensually agreed upon, or whether they have been yet identified. While the first statement highlights very specific actors that are represented and advocated by the personae of the speaker, the second statement remains largely abstract with regard to this aspect. The reference to "traditional and indigenous cultures worldwide" can in this sense be understood as a way to depersonalize the negotiated issues and to remove them from the level of individual suffering and moral imperatives. While this formalization of legal issues is common or even necessary for the legal

\footnotetext{
112 See Bhatia et al. 2005 for an edited volume on vagueness in legal texts (especially the contributions by Williams or Giannoni) for discussions of auxiliary verbs and their function in normative texts.

113 The United Nations Declaration on the Rights of Indigenous Peoples (UN 2008) uses the expression "recognizing" six times in its preamble, complemented by verbs such as "affirming", "welcoming", "convinced", "emphasizing", "considering", "encouraging" and "believing". The UNESCO Convention for the Safeguarding of Intangible Cultural Heritage (UNESCO 2003) uses "recognizing" twice as well as "considering", "noting", "referring" and "recalling". The UNESCO Convention on the Protection and Promotion of the Diversity of Cultural Expressions (UNESCO 2005) as well as the expressions "emphasizing", "recalling", "taking into account" and "celebrating". The UNESCO World Heritage Convention (UNESCO 1972) does not use "recognizing" but does use the verbs "considering", "noting" and "recalling". Expressions used in the articles of these conventions are equally "soft", because they are "soft law" with few tools to sanction breaches of convention provisions.

114 This is no normative evalutation of the EU's position, but only the description of communicative strategies.
} 
and political process, in the the IGC and in other contexts it is also useful to weaken moralized rhetorics. ${ }^{115}$

In the EU's statement, this goes along with a conception of culture as something that is primarily ideational, which neglects its social implications, functions and the material forms it is expressed in. The substantive matter discussed in the IGC is framed as less urgent and as less problematic and grave than claimed by the African Group. The way it is framed is an abstraction of specific problems repeatedly illustrated by the indigenous panel, fact-finding reports and ILCs since 2001. The EU's statement accordingly provides different categorizations of actors than the African statement, as seen by framing the actors concerned as "cultures" and not as individuals or groups of social actors. Thus, it both depersonalizes and technicalizes the discussions of the IGC. This differentiation of actor categories in the EU's statement takes place at two different levels. On the one hand, there is the realm of GRTKF to be recognized by the international community and the traditional and indigenous cultures associated with it. According to the EU's statement, this level is of lesser importance to the tasks of the IGC. On the other hand, there is the IGC with two main sets of actors: those represented by the speaker and affiliated member states (in this case, the EU and Group B), and the rest of the committee. By limiting the scope of discussions to the latter level of the IGC, the main issues and problems to be discussed are not related to the aforementioned social inequalities and injustices, but rather to the interrelations between participants of the committee who seek a "consensus" and "short-term results". Questions related to the representation of ILCs or other social actors are left out, and in contrast to the categories of actors introduced by the statement of the African Group, the focus is put on actors within the IGC.

The speaker in this instance represents the member states he is speaking for, and other member states usually associated with the speaker, which is related to the habitualized and patterned aspects of alliances of actors in IGC negotiations. These groups are relatively stable and only change due to external influences rather than being persuaded or convinced during the course of negotiations. It is thus possible for delegations to anticipate such alliances (though they might vary from forum to forum), and utterances such as those cited above accordingly imply alliances without having to be explicit. ${ }^{116}$ Substantially, the discussions are limited to an abstract level of negotiations and thus to an exchange between member states.

\footnotetext{
115 Similarly, moralized rhetorics can be understood as a useful strategy for member states.

116 An exemplary case of external influence changing the usual position of a delegation was related to me by the representative of Peru at IGC 13 (October 2008). He had wanted to make a statement in support of Chile, but was reminded by a superior that he could not do so because Peru was in bilateral negotiations with the U.S. over an issue which conflicted with the position he wanted to take. It is not easy to measure the influence of such external influences and processes. Putnam (1988) has noted the complex interrelations between domestic policy and diplomacy and they are reflected in cases such as this.
} 
Moreover, the focus of the Slovenian statement is on technical questions: it is argued that while there are areas where a consensus has already formed, the "essence of TK and TCEs" was inherently hard to define, and the methods to protect them were hard to find. By framing difficulties as inherent to the subject matter, the issues are projected away from the committee, its deliberations, and the motivations and interests of member states. Instead, difficulties are said to be part of the subject matter itself. According to this rationale, the reason why there are neither definitions of TK and TCEs nor solutions to the problems of the committee is not that there are delegations that stall the negotiations. Rather, the argument is that what hinders the IGC process is the abstract and complex "nature" of cultural property. This conceptualization allows one to see the failure of the IGC to reach consensus not as a question of blame or moral wrongdoing, but as a question of expertise, existing classification schemes and rationality. ${ }^{117}$

The technicalization also neutralizes ethical aspects of the debate and it, too, takes place at two different levels, as can be shown by the following sentences of the EU statement:

[08] It was not enough to create a definition of what was obviously TK or TCE. The line between what was TK or TCE, and what was not, had to be identified.

On the one hand, defining TK and TCEs is portrayed as inherently difficult. It depends on abstracting from social and cultural situations, which must be reduced to their main aspects and specificities so as to generalize them for inclusion in a typology of TK and TCEs cases. On the other hand, these definitions and the elicited typologies need to fit into existing legal mechanisms and frameworks. Thus, a definition of "what was obviously TK or TCE" is not sufficient as it does not meet the requirements of a legal definition.

So in addition to the description of substantive aspects of TK and TCEs, a legal definition is needed so it can be put in the context of existing legal frameworks. These frameworks exist at national, regional and international levels with regard to cultural property, as well as with respect to allied intellectual property issues. The specific legal instrument used carries its own requirements. Examples of these requirements can be the clarity of a definition, setting the object of definition apart from other objects, or by contrast, its ambiguity or vagueness if it includes a relatively large number of phenomena. The requirements are for that reason contingent on their context of use, and terms like TK and TCEs are accord-

\footnotetext{
117 The question Marshall Sahlins raised in 1976, one that has also been part of the cultural relativism debates, is to what conceptualizations of rationality and logic do we subscribe, especially in the context of intercultural research (Sahlins 1976)? Max Horkheimer's "Eclipse of Reason" (Horkheimer 1947), in a similar vein, differentiated two main understandings of reason in Western societies.
} 
ingly termini ad quem that are constructed with specific goals and uses already in mind.

The task of the committee is then seen as clarifying these technical aspects in a process that mainly relies on relevant "neutral" facts and structures of TK, TCEs and the intellectual property systems at national and international levels. Social actors only play a subordinated role in this constellation, and the factual level is highlighted. ${ }^{118}$ The "essence" of TK and TCEs ([06]) according to this conceptualization primarily consists of characteristics of certain kinds of knowledge and expression that can be isolated rather than complex social constellations and interrelations. The EU statement accordingly continues:

[09] [...] that some Members of the Committee might wish to reach a practical conclusion of its work within a certain time, for example, during the current mandate of the Committee for the biennium 2008 to 2009. Such a timely result for the work could be achieved in areas where a consensus had already been reached or nearly reached and only if flexible solutions were considered that would not bind individual countries to commitments that did not necessarily meet their needs.

The main problem diagnosed is that due to time pressures, a practical solution to some of the committee's issues could only be found in areas where a consensus had already formed. The temporal aspect, also taken up in the statement from the African Group, is modified to fit the strategy of delegations not interested in widereaching legal protection measures for GRTKF. Instead, the issue of time is used as leverage to limit the scope of "a practical conclusion" to a small number of areas the committee works on. It is not the claimed injustices and inequalities arising from the misuse and misappropriation of TK, GR and TCEs at the local and national level that cause problems for ILCs. It is the institutional time pressure caused by the IGC's status that is used as an argument for a specific mode of speeding the IGC negotiations.

Ultimately, the EU's statement proposes an alternate mode of resolving issues discussed in the IGC by moving away from the idea of a legal instrument as demanded by the African Group. This alternative as proposed by the EU's statement

\footnotetext{
118 The notion of "facticity" that is used in processes such as "fact-finding missions" and "factual extractions" is worth analyzing further. It goes along with a specific perception of what facts can be and what not. The debate on positivism and especially the contributions to it by critical theorists are helpful for understanding the difference between what is judged a fact and what is a non-fact. Briefly, the positivist stance, criticized among others by Habermas (1971), claims that the only relevant data are positive facts, and metaphysics are dismissed as irrelevant. This notion is shared by many IGC participants, influencing how indigenous perceptions of culture as holistic and spiritual (as communicated by some ILC representatives in the IGC) are addressed and dealt with in the context of a positivist and fact-based intellectual property system.
} 
would include an instrument that was not legally binding and flexible, covering only partial aspects of GRTKF: a legally binding international instrument would "not necessarily meet [the] needs" of some member states. Put differently, in the eyes of the EU it would be counterproductive for countries benefiting from the current status of the intellectual property system and not interested in a substantial change in relation to GRTKF. Moreover, the alternative instrument would have to be decided on consensually. Given that at the time this statement was delivered, there was virtually no consensus on central questions, this would have meant a very weak instrument, presumably in the form of a non-binding convention with no measures against violations against it.

By stressing a practical solution for the committee's problems, the differences that exist on substantive levels, or with regard to ethical questions, are subordinated under procedural issues. While the statement expresses that the EU wants a conclusion or a result from the IGC's work, it asks for a flexible solution. In doing so, it places the burden to compromise on the African Group asking for more binding commitments: as the EU is willing to take a decision under certain conditions that are portrayed as primarily being of a technical nature, or that are subject to factual and practical constraints, the African Group has to move away from their demands that go further. This is underlined by the end of the EU's statement:

[10] The Committee now had a great deal of information before it. Therefore, the Delegation encouraged the Committee to focus primarily on the areas where consensus was possible in the short term, in order to achieve a practical result that could be accepted by all Members.

On the basis of facts and information gathered during the IGC process, the task for the committee is portrayed as trying to elicit a possible consensus, one limited by various constraints and time pressures. Calling for a practical result on the basis of a consensus between member states implicitly argues against the far-reaching demands by the African Group. As these demands are against the interests of the EU and Group B, this call to speed up negotiations and come to specific results can be viewed as an attempt to counter the value-laden statement of the African Group without arguing against the ethical problems outlined in it: while denying the implications of the current situation for the bearers of TK, TCEs and GR would be problematic for the EU, pointing to the limits and differences in a more procedural and technical manner is possible. The call for specific results is thus a way to limit the work of the committee to a result acceptable to both stalling and speeding up delegations without arguing against the African Group as to the ethical problems of the intellectual property system.

The EU statement also includes a reference to time allotted to the three substantive items of the IGC process. An equal amount of time should be allotted to 
the discussions on GR (cf. [06]). ${ }^{119}$ In the context of IGC 12, as well as other IGC sessions, the focus of discussions was mainly on TK and TCEs. ${ }^{120}$ The call to put more effort into the discussions on GR can thus be understood as a way to limit the time for discussing the aspects of the IGC where more results have been achieved, for example in the form of lists of objectives.

The statement by the EU reaffirms the assumption that TK, TCEs and GR are important, and that the status quo has to be changed, but perhaps only by further "recognizing" their importance to indigenous cultures. ${ }^{121}$ The EU statement does not contradict IGC principles, but points to specific problems and constraints that restrict the possibilities of the IGC to achieve closure. It is a way both to make clear that one is not keen on extensively changing the existing intellectual property system - as delegates are eager to admit - and to say it in a way that makes it hard to be blamed for this, as it is achieved by conversational implicature that retains full deniability for the underlying strategy. This does not mean, however, that the EU's or Group B's strategy is not legitimate and only the proposal by the African Group is morally viable. Rather, it illustrates a situation in negotiations where one party constructs its interests as being the only legitimate position by making reference to moral aspects. Arguing against this position would imply a dismissal of these moral aspects as well, and thus it has to be countered in a different way. The EU can in this case always revert to saying that what they meant was an honest attempt at making progress, and not an attempt to argue against human rights and stall the negotiations. ${ }^{122}$ In a negotiating situation where issues of intellectual property are framed as human rights issues by one party, such implicature is a possibility to counter claims construed as being primarily about morality without having to downplay human rights aspects of the debate.

The examples examined above are only a small fragment of opening statements given during the IGC sessions since 2001. Yet, a study of the WIPO documentation, the fieldwork at IGC sessions as well as interviews with participants of the IGC have shown that the aspects outlined here are part of communicative patterns that are reproduced from session to session. The statements by the African Group

\footnotetext{
${ }^{119}$ In a statement by Japan at IGC 13 in October 2008, the delegation said equal time should be given to all three substantive items. This claim refers back to the decisions on future work from IGC 12 that states: "At its thirteenth and subsequent sessions, including any intersessional sessions, all three substantive items of the Committee's mandate should be discussed in depth and that the time allotted to each item should be balanced." (WIPO/GRTKF/IC/12/Decisions).

120 The reasons for neglecting GR are manifold but in part are due to existing, active discussions elsewhere, such as in the WTO or the CBD.

121 A proposal by the EU, issued as WIPO/GRTKF/IC/14/11 at IGC 14 in 2009, illustrates both continuity and change with regard to this question: while it is more specific in its proposed process, it is still vague as to the actual mechanism to be installed.

${ }^{122}$ Michael Silverstein calls this pragmatic strategic "diplomatic non-indexicality” (Silverstein 1976).
} 
and by the EU are two cases illustrating specific communicative forms and perspectives. The mode of reproduction of these patterns and perspectives is special insofar as it can be described as a process of instantiations. Prior utterances are not exactly reproduced modified in ways that that draw from habitualized and structural features of discourse. This modification is contingent on the context of the instantiation of an utterance, taking into account personal, strategic, temporal and structural factors that constitute the context. Other actors, strategic considerations in external fora, time pressure or constraints of the organizational frame of a meeting all contribute. One does not see a mere repetition of the same arguments and strategies, but a context-aware (not necessarily metapragmatically-aware) modification and progression influenced by idiosyncrasies, coincidences and mistakes on the part of IGC participants. This instantiation can take the form of simple repetition, but framing it only as a reproduction would miss the fact that simple repetition also implies reflexive or non-reflexive cognitive processes that result in the sameness of utterances.

The particular aspect of instantiations that reacts to or anticipates the presence of other participants in a communicative event has been termed "recipient design" in conversation analysis:

By "recipient design" we refer to a multitude of respects in which the talk by a party in a conversation is constructed or designed in ways which display an orientation and sensitivity to the particular other(s) who are the co-participants. In our work we have found recipient design to operate with regard to word selection, topic selection, admissibility and ordering of sequences, options and obligations for starting and terminating conversations etc. (Sacks et al. 1974: 272)

What Sacks et al. describe for turn-taking in conversations holds true for the drafting of IGC opening statements as well. Speakers anticipate the stance an opposing party is going to take using already available information. ${ }^{123}$ This can be in the form of prior statements, comments or proposals by a delegation, personal and professional experience and knowledge in diplomacy and the subject matter, or the broader position of a country in international processes and politics. Also, it is tied to the specificities and characteristics of the IGC as a speech community, taking into account communicative patterns, conventions, structures and dynamics that

\footnotetext{
123 See Fox 2008 for a discussion of dynamic features in accommodating to a recipient in discourse: "While, at a particular moment in an interaction, the pressures of crafting an utterance for a particular recipient, doing particular work, may seem daunting when looked at from the outside by an analyst, it is clear from research in this area that for participants this work can be accomplished, and in the speed of real-time interaction, and made to appear 'natural', 'normal', and even 'effortless'." (Fox 2008: 255)
} 
can have a significant influence on negotiations. ${ }^{124}$ It is similar to the anticipation of alliances and actor stances mentioned above, and it has the effect that statements are given already accommodating the assumed positions and the assumed strategies of the recipients of an utterance. For this reason, opening statements and other interventions are drafted dynamically and are the result of a multilayered process of recipient design, including the patterned instantiations of strategies, alliances, communicative conventions and other aspects.

A number of instantiations of communicative patterns and perspectives can be identified in many opening statements. ${ }^{125}$ The statements of the African Group and the EU are specific illustrations of more general patterns and found in the majority of statements. These can be grouped according to the specific aspects they relate to. What results is not to be understood as a typology that differentiates bounded categories, but rather as a set of generalizations that can help in the analysis of the instantiations.

These generalizations include: the technicalization of a discourse that obscures the ethical dimensions of the debate on cultural property. This is in contrast to the use of heavily value-laden references, such as to exploitation, poverty, sickness or food shortages. Accompanying this contrast, one finds either the exclusion of social actors as irrelevant for the negotiations, and as isolatable from the substantive issues or the inclusion of the bearers or holders of GRTKF as social actors, portrayed as being represented by a given speaker at the IGC. Added to this, there is a focus on facts claimed to be neutral, creating the image that negotiations are not about politics but about the objective reconciliation of interests on the basis of existing structures, such as the intellectual property system, national legislation or other legal regimes related to the substantive issues of the IGC. In contrast, there is the argument that the substantive issues discussed in the IGC heavily relate to questions of justice, fairness and morality, and that the existing legal frameworks should accommodate these principles. Time constraints are either displayed as the reason why a quick and extensive solution should be drafted in the form of a legally binding agreement and on the basis of already existing work of the committee, or as the reason why practical and consensual solutions should be found in the form of a flexible legal instrument, such as a convention that has few practical implications for member states. Two overarching strategies result: the first is speed-

\footnotetext{
124 Aspects of language policy such as translation or interpretation can also be considered part of these characteristics. Switching from one language to another during a statement is a reflexive instance of recipient design that, based on assumptions about communicability and attention, tries to elicit a non-typical reaction from the audience.

${ }^{125}$ Moreover, they can also be found in other more specific statements during IGC sessions. The distinction between opening statements and other inventions made here is due to the organizational separation. However, the expression and placement of interests are very fluid and flexible with regard to communicative patterns and conventions, as will be shown below.
} 
ing towards an agreement to change the current intellectual property system, and the second is stalling the negotiations in such a manner as to retain the status quo.

These last two aspects are not limited to the IGC or the question discussed therein. They exist in other fora and are closely linked to broader policy issues at the national, regional and international level. As the two main identifiable positions that actors take in negotiations, they are abstractions of a dynamic relationship between different member states and other participants of the IGC. They are subject to change and constitute a space within which member states position themselves: at times, some aspects might change while other stay the same. As such, the specific stance of actors is not fixed in every aspect, but may be progressively adjusted according to the changing context. For example, while stalling delegations might not agree to "text-based negotiations" in one sessions, they might accept it during the next session. However, their fundamental stance stays the same. A last factor, with the meta-quality of being explicitly metapragmatically aware, are the direct reproaches made of actors for their assumed strategies, in most cases for stalling the negotiations. These are rather infrequent and did not factor in the two statements examined above.

To look at these aspects in more detail, it is helpful to examine further examples from IGC opening statements. This shows both their diffusion as well as their dynamic and contextual modification. As these aspects are intertwined with one another, it makes more sense to group them according to their overall mood. Proceeding like this also prevents the methodological mistake of hypostatizing the various aspects as fixed tokens, when one of the important characteristics of them is their flexibility and variability, e.g. with regard to their recipient design. Taking the two overarching strategies that also distinguish the statements by the EU and the African Group as a starting point, the following illustrates how speeding up delegations and stalling delegations use opening statements to frame negotiations. The statement by Senegal on behalf of the African Group at IGC 14 in October 2009 nicely illustrates the position of speeding delegates and features many of the aforementioned aspects that contribute to the overall tone of the intervention:

[11] The Delegation of Senegal added that the other delegations were convinced that the background work already carried out as well as increased participation and dialogue would lead to tangible results. The Delegation had stated that the comparison between those expectations and the results obtained were an indication of the disappointment of the African Group as regards the outcome of the work of the Committee on the eve of the expiration of its mandate in September 2009. The Delegation stated that the African Group remained hopeful and that its interest in the issues under discussion remained high. [It] believed that the Committee could not renew the same 
terms of reference for its mandate which had led to stalemate. The Delegation underscored the holistic approach which was to lead to the effective protection of genetic resources, traditional knowledge and traditional cultural expressions, and noted the African Group had made proposals, inter alia, on the renewal of the mandate of the Committee along with a work program with a timetable for, in particular, intersession meetings as a means of accelerating the work of the Committee. The Delegation noted that the proposal of the Group referred to the negotiations based on texts [...]. The Delegation reaffirmed that only the adoption of a legally binding international instrument could guarantee the effective protection of folklore and traditional knowledge as well as genetic resources of indigenous and local communities of Member States. It expressed the wish that its proposals would, in the context of future work of the Committee, serve to correct the imbalance inherent in intellectual property at the international level with on the one hand individual property well protected by various rights (patents, copyright, related rights, ...) and on the other community-based assets at the mercy of piracy, illicit use, misappropriations or other prejudicial actions. The Delegation expressed the wish that the Committee could put a stop to that situation. ${ }^{126}$

Referring both to specific documents and methods of work on the one hand and the specific situation of GRTKF as a matter of urgency, the statement frames the upcoming negotiations as important and necessary. It pressures member states to act in a way that results in a legal instrument to change the current intellectual property system for the benefit of ILCs. The statement by the Sri Lankan delegation, speaking on behalf of the Asian Group, similarly urges the committee to move forward:

[12] It hoped that the Committee would revitalize its mandate towards a tangible result, including the possible development of an international instrument for the protection of GR, TK and TCEs. The Group expressed its ongoing concern for the misappropriation of TCEs, TK and GRs. The fundamental objective of the Committee's work was the protection and preservation of indigenous peoples' knowledge and resources. The sharing of benefits from GR, TK and TCEs should also be a guiding objective, and it was time for the Committee to have a more focused work program. ${ }^{127}$

\footnotetext{
${ }^{126}$ WIPO/GRTKF/IC/14/12:5.

127 ibid.
} 
What can be observed here is a general tone constituted by the composition of several aspects common to speeding delegation. This does not mean that interventions of this type always include all aspects. Rather, they are a composition influenced by the position of the speaker as well as the context of the meeting. As instantiations of the abstraction of one of two main positions, they contribute to the number of participants interested in progress of IGC negotiations.

Along with the stress on the urgency of the discussed issues, it is common for stalling delegations to highlight the time pressure that the committee is subjected to. In the opening statement of Zimbabwe at IGC 13, the delegate noted "with agony" the slow pace of negotiations. African countries were marginalized and the exploitation of TK, TCEs and GR as "our indigenous knowledge" had to be prevented. He argued with the legal maxim that "justice delayed is justice denied", and that in face of the urgency of issues and the sometimes relaxed atmosphere of the IGC, "seven years without an agreement are no joke". ${ }^{28}$ In a similar vein, the Nigerian delegate referred to the "urgency" of the committee's tasks due to the "exploitation" of GRTKF. According to his statement, the IGC was at a crossroads, and that a proposal by the African Group ${ }^{129}$ that outlined specific options for the "Protection of Traditional Knowledge, Traditional Cultural Expressions and Genetic Resources" was the only way to move forward.

The dynamic and context-bound character of opening statements and the specific aspects they include can be further illustrated by the interventions of speeding delegations beginning at IGC 14 in June and July 2009. Delegations from Indonesia, Zimbabwe, Iran, Egypt, Brazil, Senegal, Ecuador and Yemen mentioned the term

\section{[13] text-based negotiations}

in their opening statements ${ }^{130}$ as a signifier for an advanced stage of negotiations. According to them, the IGC was at a point where the non-committal exchange of views was not enough anymore and more tangible results had to be produced. This term was used in prior IGC sessions, ${ }^{131}$ but its use increased significantly beginning with the fourteenth session, subsequent to its use in a passage on the future work of the IGC that read:

\footnotetext{
${ }^{128}$ Author's field-notes from IGC 13.

${ }^{129} \mathrm{WIPO} / \mathrm{GRTKF} / \mathrm{IC} / 13 / 9$.

${ }^{130}$ See WIPO/GRTKF/IC/14/12 and WIPO/GRTKF/IC/15/7.

131 The official reports for IGC 13 (WIPO/GRTKF/IC/13/11) in April 2009, IGC 12 (WIPO/ GRTKF/IC/12/9) in February 2009, IGC 10 in April 2007 (WIPO/GRTKF/IC/10/7 Prov 2) show no results for the phrase; the report for IGC 11 (WIPO/GRTKF/IC/11/15) shows two results for "text-based approach", both by the delegation of South Africa.
} 
[14] The Committee will undertake during the next budgetary biennium (2010/2011) text-based negotiations on genetic resources, traditional knowledge and traditional cultural expressions. It will adopt, as set out in the Annex, a clearly defined work program and timeframe, including the holding of inter-sessional work sessions. The focus of its work, without prejudice to the work pursued in other fora, will build on the existing work carried out by the Committee and use WIPO documents WIPO/GRTKF/IC/9/4, WIPO/GRTKF/IC/9/5 and WIPO/GRTKF/IC/11/8A (TCE, TK, and GR) which is to constitute the basis of the Committees' work on text based negotiations. The Committee is requested to submit to the 2011 GA a text for an internationally legally binding instrument/instruments on TCEs, TK and GR and recommend a date for the Diplomatic Conference as agreed in its work program. ${ }^{132}$

As a change of the committee's context, the new proposal influenced delegations in the way they drafted their opening statements. It was made available to some delegations prior to the beginning of the session, and it can also be assumed that most other member states also knew about it by word of mouth or rumor. The new phrase that had been only sporadically used in prior sessions now not only redefined the tone of IGC negotiations but came to be included in the final version of the new mandate for 2010/2011.

Speeding up delegations thus used the term to frame the work done in the meetings as being primarily about deciding on text-based negotiations as a mode of work, though the term "internationally legally-binding agreement" was also used to specify the desired legal character of the outcome. The dynamic character of the aspects used in such utterances is illustrated by this example, inasmuch as the term only gained significance and a specific meaning due to the introduction of a new proposal expressing a new strategy of the African Group and other speeding up delegations.

The opening statement by Senegal on behalf of the African Group at IGC 15 in Dec. 2009 goes so far as to claim the conversational genre itself has become obsolete due to the advanced stage of negotiations. The delegate said the Group

[15] had no intention of making an opening statement in the customary sense of the term as the Group had prepared a statement focusing on what it believed to be the most salient point, namely Agenda Item 10, which was scheduled for the end of the meeting. Wishing to explain the lack of a general statement, the Delegation of Senegal declared

132 WIPO/GRTKF/IC/14/8 Rev. 
that Agenda Item 7 immediately placed the Committee at the negotiation stage, which exempted it from such statements. ${ }^{133}$

In a similar vein, the Nigerian delegate at IGC 14 noted that "we have spent the last years to congratulate the chair, then elect another chair and congratulate him again. This time I will spare this tradition". ${ }^{134}$

In conclusion, a relatively new term (at least for the IGC) was introduced and used by many delegations to frame the negotiations and set a new tone. It gained its force in the dynamic conversational interrelation between various delegations of the IGC. This example of the introduction and adoption of a specific term shows that the IGC setting can lead to specific and flexible instantiations of underlying communicative patterns and strategies rather than only reproducing existing structures. The procedural seizing of such patterns and strategies is for that reason not necessarily contingent on explicit strategic arrangement between delegations, but the result of communicative processes such as anticipation or recipient design by which alliances are formed dynamically and utterance fragments with pragmatic quality are instantiated across actors.

Direct reproaches, by contrast, are rarely used as they violate the communicative conventions and rules of conduct in the IGC. These are not documented or explicitly agreed upon, but do proliferate through the personal sanctioning of actors. One example, related in an interview, was given of a relatively young diplomat who was extremely rude in a statement and verbally attacked the delegate of another member state. After the meeting, the chairperson contacted the diplomat's superior, who had not been present during the statement, and told him about the incident. The young diplomat was subsequently admonished by his superior for his rude behavior and told to abstain from making statements for some time.

This example is presumably the exception, as communicative norms for the most part are internalized and there are other ways of expressing critique. However, various transgressions of interactional conventions do take place during IGC sessions. At IGC 13, for example, the representative of the indigenous group Tupaj Amaru directly addressed an intervention at the German delegation, claiming theirs was one of many "rhetorical statements" without much substance. Earlier during that session, he had complained about the "double standards" in the IGC, as the representative of the secretariat of the CBD spoke for almost 30 minutes whereas he had been called to order after only a couple of minutes.

His critique was understandable, as the influence of ILCs and NGOs was minimal, especially at IGC 13 . As a result, and in response to the lack of possibili-

\footnotetext{
133 WIPO/GRTKF/IC/15/7:7.

134 Author's field-notes from IGC 13.
} 
ties for ILC involvement, many indigenous representatives left the session early. The statement by the indigenous caucus announcing this boycott reads as follows:

[16] [W] are cognizant of the difficult task that you have taken on in leading this committee in its work to fulfill its revised mandate to accelerate its work. However, we must express our severe disappointment that in your haste to move into regional consultations you did not afford an opportunity to the WIPO Indigenous Caucus to express its positions on future work which could have fed into the informal discussions. This negates the very purpose of having a voluntary fund which supports indigenous participation in this committee. As you may be able to tell, some of our colleagues have already left, seeing no further purpose in simply rubber-stamping the decisions already taken. This may have been corrected by the inclusion of the elected representative of the WIPO Indigenous Caucus in the informal consultations, something which, in any case, would be required by Articles 18 and 19 of the UN Declaration on the Rights of Indigenous Peoples. [...] We will, of course, submit our now obsolete, prepared statement for inclusion in the records of the committee, and sincerely hope that the future operation of this committee will more properly reflect the rights of participation of indigenous peoples. Having not participated in the deliberations and decisions on future work, the WIPO Indigenous Caucus regrets that its inputs may not be reflected in the decisions taken by the IGC. Mr Chairman, thank you again for this opportunity to voice our concerns, and we look forward to continuing to work with you and the member states in ensuring respect and protection for the rights of indigenous peoples and local communities (reproduced from a hard copy of the statement from IGC 13).

In other cases, particular member states were directly addressed and critiqued for acts of misuse or misappropriation of TK and TCEs by commercial organizations. Such transgressions are not limited to IGCs - delegates of member states also act against communicative conventions. However, they are rare and they are, for the most part, not sanctioned but instead ignored.

Another far more frequent way of expressing critique is by way of conversational implicature. At IGC 13, the ILC Ethio Africa Union Millenium Council used the phrase "politricks" to express their view that international politics consist in large part of trickery. The Peruvian delegate made the indirect accusation that there had been "five years with no progress", and that the committee was losing time with the "same chit-chatting as always". The delegate from South Africa noted that one cannot just "come home with outcome-oriented deliberations". 
This manner of criticizing other delegations, groups of delegations or specific strategies by implying something without explicitly saying it is quite common in the IGC. It is also part of the aforementioned statements by the African Group who referred to the "stalemate" of negotiations (see [11]). The speaker and associated participants were portrayed as active, implying others were being passive, and the misuse and misappropriation of TK and TCEs were criticized with the implication that those who do not act against this are complicit in the misuse. Reproaches, either in the direct form or by conversational implicature, frame negotiations and are contingent on having a recipient in mind.

The position of stalling delegations can be exemplified by a number of opening statements along the lines of those already outlined above. Similar to the interventions by speeding delegations, one can find instantiations of communicative patterns and strategies adjusted to their specific context. They contain the framing of the subsequent meeting and foreshadow the position a delegation is going to take. At IGC 14, for example, the delegation of Germany, speaking on behalf of Group $\mathrm{B}$, and following a typical initial sequence stated that

[17] TK and TCEs had taken on new economic and cultural significance within the globalized information society. GRs had assumed increasing economic, scientific, and commercial value to a wide range of stakeholders with the emergence of modern biotechnologies. The conservation, sustainable utilization, and benefit sharing were already being addressed within a range of different policy areas and forums, including food and agriculture, biological diversity, biotechnology, public health, and economic development. The Delegation said that the contribution of TK and GRs to tackling global challenges would be reflected in discussions during the WIPO Conference on Intellectual Property and Public Policy, which would take place in July 2009. The Committee had a leadership role to play in addressing the IP aspects of the protection, promotion, and preservation of TK, TCEs, and GRs. In particular, Group B hoped for progress in identifying the policy objectives and principles for protection of TK and TCEs. It wanted to deepen its understanding of the issues related to TK and TCEs, and of the possible gaps in TK and TCE protection. [...] It agreed with other Delegations that the Committee could assist in bridging gaps in protection by producing tangible outcomes, such as guidelines and recommendations. Group B also hoped that the Committee would enhance its work on GRs. Concerning the international dimension of protection, it said that it was looking forward to discussions on the IP aspects of GR, TK and TCEs. The Committee should focus its discussion on the IP aspects, as a common understanding of these as- 
pects would substantially advance the international dimension of the Committee's work. ${ }^{135}$

Included in this statement are aspects typical of stalling delegations. Among them are shades of an generalized affirmation of the process and a valuation of GRTKF as instrumental for various processes. ${ }^{136}$ The statement mentions "a wide range of stakeholders" as the relevant social actors for the process. This implies that the construction of TK and TCEs as belonging to ILCs who are represented by some of the member states is contested. In conjunction with the reference to the various fora already discussing cultural property issues, the statement also delimits the scope of WIPO deliberations solely to intellectual property aspects. By using unspecific and pragmatically weak phrases such as "to deepen the understanding", "could assist", or "addressing the IP aspects", the statement expresses a general willingness to participate in the work of the committee, but abstains from making concessions to the demands of the African Group, most notably the demand for a legally-binding international instrument. The flexibility or softness of verbs reveals the speaker's position for the subsequent negotiations: he is unwilling to concede to claims by speeding up delegations, but does so without being too open and direct about it. Here, the non-pragmatic character of the statement with regard to progress of the committee is contrasted by a highly pragmatic conversational implicature clarifying the stalling stance of the delegation. Finally, by implying that a "common understanding" is lacking as of this session, the speaker justifies his stance: the understanding has to be deepened by further discussions, as a consensus cannot be found. This is complemented by the expression "the possible gaps in TK and TCE protection" that questions the existence of such gaps until they are proven by the technical work of the committee. The non-specificity of the statement as to the specific steps to be taken by the IGC can thus be understood is a way of stalling the negotiations. It is an example of recipient design and the instantiation of underlying communicative strategies and patterns, related to the statements of speeding up delegations by affirming some aspects of them, such as the general appreciation of TK, TCEs, and GRs and the possibility of gaps in their protection, while contesting others, meaning a shared understanding of intellectual property aspects.

With regard to text-based negotiations, the statement by the Singapore delegation at IGC 14 illustrates how stalling delegations justify their reservations against this specific mode of work:

\footnotetext{
${ }^{135} \mathrm{WIPO} / \mathrm{GRTKF} / \mathrm{IC} / 14 / 12: 6$, my italics.

136 This conceptualization of TK and TCEs as resources for modern technology is noteworthy and one possible perspective on the substantive issues discussed in the IGC.
} 
[18] However, it expressed concerns with some elements in the proposal, such as moving to text-based negotiations given the present lack of consensus on many fundamental concepts, the limited range of issues for discussion, the proposed timelines, and the use of a restricted range of documents for the Committee's work..$^{137}$

It is argued that this approach would have negative influences on the work of the IGC as it was too early to proceed with a more focused method of work. However, the reason given is not a lack of motivation or willingness to move forward, but a lack of consensus and understanding. In a similar vein, the U.S. delegation at IGC 14 argued that

[19] [...] much work remained to be done at the international level including the unfinished analytical work of the current biennium. [T]he Delegation believed that the acceleration of the substantive work of the Committee held the greatest promise of reaching the consensus that was necessary to advance its work. [N]o outcome of the Committee, including the adoption of an international instrument or instruments addressing the preservation, protection and promotion of TK, TCEs and GR, should be excluded and, similarly, no outcome should be prejudged. [...] The African Group was thanked for tabling its thoughtful proposal for the renewal of the mandate and the Delegation was reviewing it closely under the general positions and principles that have informed its participation in the Committee. It was interested in learning more about the proposal from its proponents, about the views of other delegations, and about the financial and administrative implications of the proposal from the Secretariat. [...] The list of ten issues on both TK and TCEs provided a useful framework for facilitating the kind of sustained and focused discussion of these issues needed to reach a consensus on these important issues. The Delegation continued to believe that the Committee had begun to make some progress in identifying the possible gaps in the existing framework for the protection of TK and TCEs, including identifying certain options that the Committee might wish to consider addressing any such identified gaps. At its last session, however, the Committee barely scratched the surface of its deliberations on this important topic. Much work remained to be done. Against a background of high expectations, the Delegation shared the frustration of many other Delegations with the slow progress of the Committee over the last two years. Nonetheless it looked forward to a positive outcome at the fourteenth session and would be willing to support a

${ }^{137}$ WIPO/GRTKF/IC/14/12:20. 
recommendation to the General Assembly to renew the mandate for another two year period. ${ }^{138}$

The typical aspects outlined above are included in this statement. However, the most interesting aspect is the framing of stalling delegations as unsatisfied with the situation as well. The reasons for the slow progress are said to be the nature of the substantial issues as well as analytical work that has to be done in order to proceed to the next stage of negotiations. The argument is that technical issues hinder the adoption of a legal instrument, and that the U.S. delegation and other Group B states are as interested in finding a way to protect the TK and TCEs of ILCs as the typical speeding delegations.

Yet, implied in phrases like "being interested in learning more" and "looking forward to a positive outcome" is the rejection of the demands of other delegations and their demands: Group B is critical of a new international legally-binding agreement, but openly telling the African Group their demands will not be met would likely result in more direct confrontation. Using implicature is thus a way to convey this position more subtly and less confrontational. The combination of portraying the work of the IGC as unfinished ("barely scratched the surface of its deliberations on this important topic", "much work remained to be done") on the one hand, and expressing the hope of making substantial progress on the other is indicative of such a strategy. Judging from the reactions by other delegations to such statements and drawing from conversations and interviews with participants, most delegates are aware of this strategy. Yet, it constitutes a documented position of the specific delegation that is hard to contest. On the surface, delegations in this manner do offer a positive evaluation and an expression of willingness to achieve progress. This is the advantage of conversational implicature or "diplomatic nonindexicality" (Silverstein 1976): the assumed indexical meaning of the statement is only that, assumed. The speaker of an utterance can thus not be held directly accountable for its implied pragmatic aspect, as the actual semantico-referential meaning is hard to criticize.

At IGC 15 the Swedish delegation, speaking on behalf of the European Union and its member states, was confronted with having the term "text-based negotiations" included in the new mandate. The delegation expressed that it

[20] [...] looked forward to a constructive, efficient and fruitful meeting. It said that it remained committed to making progress in the important issues under the Committee's agenda and attached great importance to its work. It acknowledged that the Committee had a leading role in addressing the IP aspects of protecting, promoting and preserving TK, TCEs and GRs, and strongly welcomed the decision of

${ }^{138}$ WIPO/GRTKF/IC/14/12:19-20. 
the General Assembly to renew its mandate. It hoped that the renewed mandate would imply an accelerated and more constructive work within the Committee. It recalled that the discussions had for some time focused on the issue whether the outcome of the Committee's work should be legally binding or not and stated that the renewed mandate allowed for both options. It added that the Committee's work needed to get some positive momentum by constructive discussions on basic substantive issues. It said that once a broad agreement in substance would be reached, the Committee could come back to the issue of the legal character of the legal instrument or instruments that the Committee was requested to submit to the 2011 General Assembly. [...] It recalled that the Committee had worked on the interplay between intellectual property and TK, TCEs and GRs for more than eight years and that significant achievements had been made during this time. It was however of the view that a lot of work was still to be carried out. Many difficult questions regarding the essence of the sought protection and its interplay with existing intellectual property rights needed to be analyzed and answered. ${ }^{139}$

Again, this statement shows common aspects for stalling. It also highlights the significance of a contextual analysis of these aspects, as it makes reference to the new situation by modifying the instantiation of an underlying stalling strategy: given that the mandate for the IGC was renewed by WIPO's GA prior to the meeting and that the new mandate included "text-based negotiations" as a mode and that work on drafting an "international legal instrument" " was necessary, stalling delegations had to adapt to this new situation. Stalling tactics used at prior IGC meetings would not have been sufficient to counter this strong mandate. What can be observed then are attempts to reframe the wording of the new mandate in order to make it less binding and more favorable for stalling delegations. This is achieved by arguing that the question about the specific legal nature of a possible instrument would hinder a quick agreement in the IGC, and that this question could be settled at a later point of time. Moreover, it is stressed that the committee has already achieved a lot of substantial results, thus taking away some of the pressure.

\footnotetext{
${ }^{139}$ WIPO/GRTKF/IC/15/7:10.

140 The final text of the renewed mandate is similar to the proposal by the African Group at IGC 14 ([14]). It includes the main aspects of this proposal by keeping the phrases "text-based negotiations" and "international legal instrument" with some modifications that can be described as the result of a micro-editing process: "The Committee will, during the next budgetary biennium (2010/2011), and without prejudice to the work pursued in other fora, continue its work and undertake text-based negotiations with the objective of reaching agreement on a text of an international legal instrument (or instruments) which will ensure the effective protection of GRs, TK and TCEs." (WIPO/GRTKF/ IC/15/Ref. Decision 28)
} 
In conclusion, opening statements consist of a variety of different aspects reaching beyond the initial sequences. They are both a possibility for the speaker to pave the way for subsequent negotiations, and for the listeners to gain insight into the position and strategies the delegation will follow. It appears that uttering the greeting has become a sign of accepting the nature of the speech community's rules, a signal that one is willing to play by those rules, as well as a speech genre that allows one to express attitudes toward potential results or even just the generating of results without having to be specific.

Aside from the functions of initial sequences, opening statements contribute to framing different positions and strategies at the beginning of a session. By stressing terms such as "practical", "effective", "comprehensive", and "harmonious" in their greetings, some delegations signal their interest in making progress, others go further and demand more speed through "improved results" or "tenable progress". Yet others are more interested in stalling, such as Group B who during the February 2008 meeting included in their opening intervention the phrase that it was important to them "to continue the discussion and learn more" - that is, a leaning back and waiting approach. ${ }^{141}$ Opening statements thus consist of recurring and flexible patterns and strategies contingent on their specific context, usually drafted in anticipation of a recipient's position and reaction. During IGC meetings, the dialogic quality of opening statements results in the construction of oppositions, primarily between member states interested in progress and others not interested in changing the status quo. This construction is the result of communicative interaction and opposing interests of state actors rather than a planned and intentional process. These oppositional perspectives are framed in the process of statements by instantiations of superordinated communicative patterns and strategies, adapted to the specific needs and configurations of a situation.

Part of this framing process are "shifters" (Silverstein 1976:24), referential indexes whose reference shifts regularly, depending on the contextual variables of a communicative event. ${ }^{142}$ According to Silverstein, the referential value of a shifter "depends on the presupposition of its pragmatic value". That one is interested in learning more about some specific issue, for example, includes the presupposition that one has already learned about this issue before. The dependence on contextual information and presuppositions by many participants of a communicative event thus causes uncertainty as to the specific pragmatic and referential meaning of an utterance. This is because the meaning of shifters changes from context to context, but also from recipient to recipient. "Interested to learn more" can mean that

\footnotetext{
141 Aside from the implicature of such an expression, it is of course a legitimate argument to request extensive discussions and to try to reach a consensus on many levels. Likewise, "stalling" the IGC process is not to be understood as a normative evaluation, but it is - just as "speeding up" - a legitimate position for state actors to take.

${ }^{142}$ Silverstein notes that these shifters are called "duplex signs" by Jakobson (1971).
} 
the speaker really wants to learn more, but at the same time a contextual analysis of this utterance elicits its pragmatic value that the speaker wants to stall the negotiations. Yet, on the semantic level this pragmatic value can be denied. The indeterminacy and variability of shifters makes it possible to use them as part of pragmatic strategies for opening statements, for example allowing a signaling of position to the recipients of a communicative event without explicating the implications and presuppositions of an utterance.

This can be illustrated by two examples based on the diverging interests of delegations. First, speeding up delegations often refer to indigenous and local communities as social actors suffering from the misuse and misappropriation of their TK, GR and TCEs. In opening statements, this is one of the key arguments of speeding up delegations for the validity of their demands, as well as for the urgency and importance of a legally-binding instrument. Yet, this position is accompanied by the presupposed view - at least for many African countries - that in the last instance there are no specific indigenous groups in their countries because the majority of Africans are indigenous. Accordingly, cultural property was to be held and managed by the "competent national authorities"143 and not by autonomous indigenous organizations. This position was heavily criticized both by ILCs and Group B member states holding the view that indigenous groups should be the main beneficiaries of a potential legal instrument. The proxy-representation of ILCs by member states, including the pragmatic value of the state as the quasibeneficiary, was to some extent veiled by the referential value of the statement that urgently called for the adoption of a legal instrument to benefit ILCs.

Second, stalling delegations frequently stress their willingness to contribute to the discussions and achieve progress. These utterances are in most cases accompanied by reservations as to the specific approach and technical possibility of coming to a quick conclusion. A lack of shared understanding, outstanding analytical work or other constraints are cited making it problematic to come to an immediate consensus. Again, these utterances function as shifters that have different contextual meanings on the pragmatic and referential level, as well as the capability to veil the strategic dimension of an utterance to some extent.

How can the function of such shifters in international negotiations such as WIPO's IGC be best explained? I argue that what Urciuoli calls "strategically deployable shifters" is a useful concept for understanding these processes. Using the example of specific terms such as "multiculturalism" in college marketing, she examines their pragmatic quality in relation to the specific contexts they are used in:

\footnotetext{
143 This phrase is part of the "Draft Articles on the Protection of Traditional Cultural Expressions Prepared at IWG 1" (WIPO/GRTKF/IC/17/9) from September, 2010. The document was discussed in depth at IGC 17 in December 2010. The phrase "the competent authority" was part of the section on the management of rights and was mainly defended by African delegations with the rationale that GRTKF was to be managed by the state. However, this was not argued openly.
} 
In these institutional registers and discursive fields, terms like skills, communication, leadership, citizenship, multicultural and diversity can serve as strategically deployable shifters, or SDSs. By this I mean a lexical item or expression deployed in different discursive fields so that, in effect, people using term $\mathrm{X}$ in a referring expression in field $\mathrm{A}$ are engaged in a different pragmatic activity from those using the formally identical term $\mathrm{X}$ in a referring expression in field $\mathrm{B}$. The salient interpretation of the term depends on the relation of its user to its audience and so shifts with context; in that sense SDSs have shifter-like qualities. These qualities are most evident in the differential deployments of multiculturalism in various education-associated fields [...]. (Urciuoli 2000: n. pag.)

The strategic dimension to these shifters is of importance to specific terms and utterance fragments in the IGC as well. Their distinctiveness in this context lies not only in "shifting" between contextualized referential and the pragmatic meaning of an utterance, but also in the strategic use and conscious choice to employ these terms. Thus, the phrase "text-based negotiations" is contingent on the context of its use and the speaker who uses it. It can mean various things, but used by a speeding up delegation at a specific point of time, it refers to a very specific presupposition that implies the drafting of a treaty or convention. Similarly, phrases like "to deepen the understanding", or more generally the use of "soft" verbs with little pragmatic implication ("to recognize"; "to consider") reflect certain strategies of stalling delegations. However, their specific meaning and interpretation by recipients depends on the context of the utterance or "the relation of its user to its audience" (Urciuoli 2000: n. pag.). They are strategically deployed in negotiations, e.g. in order to establish certain frames, to rebut the arguments of opposing parties, to doubt the willingness of other delegations to achieve progress, or to question the saturation of negotiations. Such shifters realize a contextualized signaling function that hints at realms of shared understanding between groups of participants or alliances. Aside from their semantico-referential meaning that is, as Urciuoli argues, often denotatively empty, there is a two-fold indexical meaning. Terms like "text-based negotiations" are powerful not only because they denote a specific concept, but because of their indexical and pragmatic values. An affirmative use of the term highlights the strategic position of actors who want to exert pressure and it signals this position to other delegations. The substantive framing in terms of general position of a delegation is thus accompanied by a strategic framing outlining possible actor positions to be taken in negotiations. The substantive framing in terms of general position of a delegation is thus accompanied by a strategic framing outlining possible actor positions to be taken in negotiations.

Strategically deployable shifters are not limited to separate terms. They also include specific phrases and expressions and even syntactical constellations such as "analytical work", "exploitation" and "piracy", "to continue the discussions and 
learn more" as well as the combination of soft verbs with relatively strong nouns (as in "to recognize the importance"). Their meaning is contingent on their specific context which includes substance, strategy, as well as the relation of a given speaker to his audience. They establish different referential frames and discursive fields that allow for pragmatic strategies often only thinly veiled, such as expressing commitment for the process while arguing for the impracticality of an agreement. Shifters are embedded in opening statements drafted in anticipation of a specific audience that consists of possible allies and opponents. When pointing to a shared understanding of substantive questions and shared strategic positions they index possible alliances. As such, shifters can be described as "pivotal devices" within these statements, realizing different indexical functions. By indexing negotiating positions, they differentiate between actors and interests and refer to perspectives with regard to the substantive issues negotiated in the IGC. As they are shifting depending on context, it is necessary to reflect this flexibility and resulting ambiguity at the analytical level. From the perspective of IGC participants, it is necessary for the speaker of an utterance to take the context into close consideration, as a shifter is only conducive to one's interests if it is used in the "right" way.

The specificity of shifters for opening statements adds to the overall importance of this conversational genre. Not only does it function to acknowledge and accept norms, conventions and organizational constraints, give insight into a delegations' position, and to prepare strategies and legitimate them. It can also - depending on the shifter or the composition of shifters that is used - signal general or specific actor group positions. Shifters not only differentiate between positions, they also map commonalities and common ground between actors who otherwise hold incommensurable positions. They also mediate between different actors, contribute to the cohesion of the committee and allow for the expression of fundamentally different viewpoints - and this is the foundation of the possibility to negotiate. This is of course not to say that they enable progress in international negotiations. However, they are a way for actors to exchange highly divergent views while at the same time creating a discursive space that allows this exchange.

Utterances recognizing the importance of TK, TCEs and GR exemplify this, and both representatives of stalling and speeding up delegations use them. Looking at them in context and in relation to the speaker, the first group of actors makes use of them to create a basis for stalling. As they affirm the general goal of the committee and highlight the importance of their work, stalling strategies can be legitimated by a technicalized discourse that projects the reason for stalling onto procedural and technical questions such as a lack of analytical work. Those who wish to see more rapid progress use these utterances and the ideational foundation they create to argue for a quick agreement and the implementation of their claims. Even the use of IGC terminology such as "traditional knowledge", "traditional cultural expressions", "genetic resources" and "indigenous and local communities", - contributes to the creation of common ground and a degree of shared 
understanding. While there is thus the expression of commonalities implied in these utterances, they also include a more specific reference that differentiates between groups of actors, strategies and goals. How opening statements are composed leads to this situation that both stresses basic commonalities and emphasizes nuanced distinctions. Situated at the beginning of an IGC session, they establish different referential frames as well as an underlying sphere that is agreed on and enables the discussions to proceed. Furthermore, they signal alliances and create opposing actor positions by including strategically deployable shifters.

For this reason, opening statements can be conceptualized as "boundary objects". The term stems from the work of Susan Leigh Star und James R. Griesemer (1989) who analyzed communicative processes among actors connected to Berkeley's museum of vertebrate zoology. Their question was how social cohesion could be maintained, given the wide variety of actors and viewpoints. They found the situation was managed by using boundary objects:

Boundary objects are objects which are both plastic enough to adapt to local needs and constraints of the several parties employing them, yet robust enough to maintain a common identity across sites. They are weakly structured in common use, and become strongly structured in individual-site use. They may be abstract or concrete. They have different meanings in different social worlds but their structure is common enough to more than one world to make them recognizable means of translation. The creation and management of boundary objects is key in developing and maintaining coherence across intersecting social worlds. (Star and Griesemer 1989: 393)

The combination of opening statements that include shifters and other instantiations of communicative patterns and strategies also features in the construction of such boundary objects. They enable actors from "intersecting social worlds" to maintain a coherent and stable identity, in this case of the IGC as a speech community, while encompassing diverging and incommensurable viewpoints. The resulting speech community is flexible enough to include these different perspectives as it draws from a coherence established by common factors, terms and viewpoints. Boundary objects, in the context of the IGC, mediate between the two main positions of stalling and speeding delegations.

Neither boundary objects nor strategically deployable shifters and the recipient design of opening statements are static, but are subject to dynamic processes influenced by the composition of actors and strategies. Boundary objects and shifters can signal a specific stage in negotiations, as illustrated by the phrase "text-based negotiations". This flexibility is also the result of how young the IGC is. If the speech community cannot draw from an longer history and stable communicative conventions, shifts in meanings and understandings are more likely to happen. At the same time, it is part of the nature of international negotiations that continual 
attempts are made to redefine and reframe the context to gain an advantage in position.

A counter-proposal by the European Union illustrates this point. It was formulated in reaction to a proposal by the African Group ${ }^{144}$ at IGC 13 in October 2008. The proposal was referred to in the EU's opening statement and included a relatively strongly-worded preamble uncommon for stalling delegations:

[21] The IGC acknowledges the intrinsic value of these issues which are also important in global trade and economic development. Indigenous and local communities have the right to maintain, control, protect and develop their intellectual property over such cultural heritage, traditional knowledge, and traditional cultural heritage. In this respect, the IGC will prepare a declaration on the value of traditional knowledge, traditional cultural expressions and genetic resources and their protection against misappropriation to be adopted by the WIPO General Assembly. ${ }^{145}$

The relative clarity and openness of this preamble, including an uncommon reference to the misappropriation of GRTKF, is primarily possible because the beginning of the proposal is followed by rather weak paragraphs that do not mention text-based negotiations and use the phrase "outcome-oriented deliberations" instead. They also do not make any larger concessions to demands by the African Group.

However, the shift from phrases like "to recognize the importance" to a critique of misappropriation is noteworthy, as it illustrates the awareness of stalling delegations that the specific context of IGC 13 called for a different manner of framing the subsequent negotiations. Using less strong wording would have led to a lack of boundary objects constituting a shared sphere mediating between stalling and speeding delegations. As a strategically deployable shifter, this wording is still mostly semantically empty.

By modifying the text of an opening statement, the EU was able to provide an instantiation of an underlying communicative strategy that fit the specific context of negotiations. For that reason opening statements contribute to the processes of negotiating cultural property within the IGC in a significant way. Their analysis reveals communicative patterns, conventions and strategies that are valid for other communicative events of the IGC and other international fora.

The $16^{\text {th }}$ session of the IGC was the last to include opening statements on its agenda. Since IGC 17 in December 2010, the procedure of these long-drawn initial

\footnotetext{
${ }^{144}$ WIPO/GRTKF/IC/14/8 Rev.

${ }^{145} \mathrm{WIPO} / \mathrm{GRTKF} / \mathrm{IC} / 14 / 11$.
} 
statements has been abandoned. ${ }^{146}$ This was part of a larger attempt by the chair of the 2010-2011 biennium, Ambassador Philip Richard Owade of Kenya, to move negotiations ahead. In his opening remarks of IGC 17, he announced that he would put more stress on the observation of time allotted to agenda items and be very punctual about keeping to this schedule. He hoped "to continue with the journey" and the "course we have set", that there was a "momentum to be maintained" and a "chartered out course with time-lines" for this "working meeting". The "positive dynamism" of the first inter-sessional working group (IWG 1) and its great success should be maintained by "making the most effective use of time":

Focus was therefore required and any attempts to detract or sidetrack the Committee had to be resisted. He implored the Committee to collectively chart out the most direct path to its destination. As Chairman, he would help to steer the process and, in doing so, endeavor to exercise fairness and impartiality. However, coming from a developing country in Africa, he could not be oblivious to the desires of millions of people from his continent and other developing countries for whom a legally binding instrument for the protection of GRs, TK and TCEs should already have been concluded. There was no doubt that the Committee had exhausted the preliminary deliberations and needed to move straight to the norm-setting mode. A legally binding instrument was long overdue to combat bio-piracy that had proceeded unchecked for decades. ${ }^{147}$

Thus, there were "no opening or general statements", the sessions would continue during regional meetings, and there were no official tea or coffee breaks. A "precise working methodology" and a focus on the "substantive work of the meeting" were to achieve tangible results (author's notes, IGC 17). ${ }^{148}$

Though opening statements have been abandoned, the associated communicative patterns and strategies are still present. Indeed, an earlier attempt to change communicative habits, namely during the IGC 12 in early 2008 was not notably successful. After more than two days of opening statements, the chair of the session decided to switch into an "informal mode" of negotiations. Yet, subsequent statements did not differ significantly from previous ones, with parts of the initial sequence reproduced by delegates, though by that point, most member states had already made their statements.

\footnotetext{
${ }^{146}$ See WIPO/GRTKF/IC/17/INF/3 for the agenda of IGC 17.

147 WIPO/GRTKF/IC/16/8: 4.

${ }^{148}$ In an interview with the delegates of Switzerland, they stated that the chair's influence can be significant in negotiations, both in formal and informal sessions, but that this was also contingent on the chair's competence and way of chairing the session.
} 
When asked, the chair said the "informal mode" was chosen to encourage national delegations to engage in "a more flexible and vivid discussion". Delegates were not to repeat themselves and limit themselves to "new" statements, though this, too, was not adhered to.

In the EU's internal consultation, delegates were baffled as to what the chair wanted, nor did most of them understand what this "informal mode" entailed. However, some delegates did note that it was common to switch to an informal mode to speed up negotiations with regard to coming to a decision about future work to be done. Both delegates and the chair thus were (and are) cognizant of the inefficiency of the conversational genre being used. The underlying communicative patterns are found in other communicative events as well. One reason for this clearly is that some delegations do not mind this inefficiency, or even deem it helpful for their interests. Patterns and strategies of opening statements therefore are both common and significant for the IGC in its entirety, and they persist although the conversational genre as such does not exits anymore.

\section{Referential Frames in the IGC}

Actors in the IGC face a process-oriented component of communicative events within the speech community that leaves them partly unsure about the events' conventions. They nonetheless have to take in account already established patterns, both in opening statements they make and in other conversational genres. The indexical features of specific utterances are thus only in part collectively understood and hence viewed as binding. The committee itself, however, produces text, including written decisions on future work the committee is to undertake, proposals for the wording of future agreements, or definitions of the core items under negotiation. There are also written reports of the sessions that reproduce, almost verbatim, statements that are made during the meetings. The written documentation, in particular, gives the language used in the IGC its binding character.

Committing to a specific phrase in official decisions taken by the IGC creates a fixed point of reference. Participants in the speech community who are opposed can use it to their advantage or disadvantage during subsequent meetings, for example to speed up or stall the proceedings. A number of such indexical markers can be heard during the sessions, including:

[22] taking into account the previous work of the IGC

[23] without prejudice to any outcome

[24] text-based negotiations 
These devices blur and burden any future work of the committee by making reference to existing yet not adopted and thus controversial texts [22], by not making concessions to any form of potential legal instrument [23], or by making a commitment to a specific kind of negotiation procedure that can lead to a binding agreement [24].

What they share is that they are based on the contingencies of linguistic rules of interaction and interpretation. Analyzed without knowledge of the context, these examples appear to be "neutral" phrases without much impact. Of course a committee in existence since 2001 should, seven years later, not have forgotten all the substantive work it has carried out. Of course, negotiations should remain open, avoid lock-in effects and consider different outcomes. Of course, text-based negotiations have the advantage of being more transparent, open to scrutiny and reflecting the opinions of all participants. Yet, these phrases have indexical meaning and pragmatic implications that can make them - depending on what one's stakes and interest in the IGC process are - less desirable for participants in speech acts than they appear to be at first sight. Bakhtin uses the notion of "typical expressions" to describe a patterned form of the recurrence of certain phrases in speech genres that during the course of repeated communicative interaction have taken on a specific semantic or pragmatic meaning:

When we select words in the process of constructing an utterance, we by no means always take them from the system of language in their neutral, dictionary form. We usually take them from other utterances, and mainly from utterances that are kindred to ours in genre, that is, in theme, composition, or style. Consequently, we choose words according to their generic specifications. A speech genre is not a form of language, but a typical form of utterance; as such the genre also includes a certain typical kind of expression that inheres in it. In the genre the word acquires a typical expression. Genres correspond to typical situations of speech communication, typical themes, and, consequently, also to particular contacts between the meanings of words and actual concrete reality under certain typical circumstances. Hence also the possibility of typical expressions that seem to adhere to words. This typical expression (and the typical intonation that corresponds to it) does not have that force of compulsoriness that language forms have. The generic normative quality is freer. (Bakhtin 2007:87)

This holds especially for phrases such as "text-based negotiations", which after repeated use take on the character of typical expressions. At the same time, these expressions and their meaning depend on a shared understanding and shared norms of interaction and interpretation. 
In situations of contingency, such as in a relatively new speech community that brings together diverse actors, the interpretation of the semantic and pragmatic meanings of such expressions is unstable. It can vary depending on context, speaker and recipient, and its meaning can be contested by various actors. This instability or indeterminacy of meaning can be both advantageous and disadvantageous for actors. On the one hand, if the utterance of a typical expression has the desired effect, the speaker can affirm the prescribed meaning. An example can be seen in the following statement by Zimbabwe that was made in the context of discussions on the future mandate of the committee for the 2010/2011 biennium at IGC 14:

[25] My limited knowledge would only confine me to refer those delegations who have concerns with the term text-based negotiations to review or revisit UN language. It is a common terminology. Right now what we are doing, we have a text in front of us - that's textbased negotiation what we are doing. There's a text in front of us we are negotiating to improve the text that is there. And paragraph 3 clearly spells out the recommendations that we will be looking at. So if delegations have problems with the documents cited, they should not hide behind the finger, pretending not to understand the term, a UN terminology, text-based negotiations. For me it's ironical and it's unacceptable. It's difficult to understand what we are doing here if certain delegations think that they don't understand whilst they understand a UN terminology which is commonly used everywhere. ${ }^{149}$

Here, the repeated usage of the term "text-based negotiations" in UN negotiations is used to bolster the argument that it is indeed a fixed term with clear implications as to the procedures of such negotiations, and that deliberations about the specific meaning of the term are primarily efforts to sabotage the work of the committee. According to an Egyptian diplomat based in Geneva, the concept of text-based negotiations unambiguously denotes the drafting of a text as the basis for a treaty or a convention; the term signals an advanced stage of negotiations with available documents that can function as reference texts for a draft text. ${ }^{150}$ Instead of a noncommittal exchange of opinions and positions on the substantive issues under negotiations, in text-based negotiations text is actually drafted and agreed upon.

\footnotetext{
${ }^{149}$ Statement on WIPO/GRTKF/IC/14/8 Rev. by the Zimbabwean delegate at IGC 14, 1 July 2009.

150 Personal conversation at the Conference on Intellectual Property and Public Policy Issues, Geneva, 14.07.2009.
} 
In [25], the delegate from Zimbabwe is referring to documents ("recommendations") proposed by the African Group to be the basis of negotiations. ${ }^{151}$ The indexical meaning of the term "text-based negotiations" within the UN system is both emphasized and is claimed to be commonly used. The delegate argues that "text-based negotiations" means, as a matter of course, negotiations based on a given text, and that is what a committee working towards an agreement should do. Pointing out the shared use of the phrase and its conventionality is a means for participants of the speech community who want to speed the proceedings and reach an agreement to put pressure on delegations who are unwilling to agree on this specific method of negotiation. Having an exchange of opinions, commissioning fact-finding missions and gap analyses, or deciding what should be done during the next committee meeting is far less binding than actually working on specific paragraphs of a potential legal instrument. "Continuing the discussion" and "learning more about the issue" are the corresponding phrases that signal a stalling position, where advancing to the next stage of negotiations - towards some sort of agreement - is not wanted.

Arguing that "text-based negotiations" are a common term and a common approach in international negotiations is a way to impose, or foster, a process on other participants. It is a communicative strategy used by the African Group to strengthen their demand for a legally binding instrument: if the ordinary sequence in committees like the IGC is to proceed on to text-based negotiations after extensive phases of fact-finding, analysis and oral negotiations, then rejecting this phase of doing so can be discursively portrayed as a refusal to move forward, and thus to stall the process. To "continue discussing" and "learn more" would in this context not primarily be viewed as interest and willingness to broaden the knowledge-base of the committee, but rather as a signal that one does not concur with the demands of the opposing party. In this specific case, it is a way for Group B and the EU to say that they are not willing to give in to the African Group's demands and commit to a legally binding agreement.

In the context of the specific example given, the discussions were about the text for the new IGC mandate to be decided by WIPO's general assembly. It was thus of considerable relevance that what would be included in the text had to be agreed upon by the committee, as this would determine the work to be done by the IGC during the next two years. If "text-based negotiations" can be framed as a "natural" subsequent step in committee meetings, the rejection of such negotia-

\footnotetext{
151 WIPO/GRTKF/IC/14/8 Rev., para. 3: "It will adopt, as set out in the Annex, a clearly defined work program and timeframe, including the holding of intersessional work sessions. The focus of its work, without prejudice to the work pursued in other fora, will build on the existing work carried out by the Committee and use WIPO documents WIPO/GRTKF/IC/9/4, WIPO/GRTKF/IC/9/5 and WIPO/GRTKF/IC/11/8A (TCE, TK, and GR) which is to constitute the basis of the Committees' work on text based negotiations."
} 
tions would thus not only be associated with a position of seeking clarity on issues, but also with blocking the decision about the new IGC mandate. ${ }^{152}$

A way to establish the conventionality of a term or a phrase when it is being contested by some participants in the speech community is to underline its previous use by the opposing party. If they used the term in the past, arguing it was common to international negotiations, asking for clarification of its exact use appears misleading and spurious:

[26] As to my colleague who wanted to know what we meant by textbased negotiations: I would remind you that in other fora the EU has proposed text-based negotiations as a phrase. And in many negotiations this phrase is used, text-based negotiations, and now we are being asked to define it when it is in common use. ${ }^{153}$

Here, the Angolan representative, as a member of a speeding up delegation, responds to a question by the EU and other delegations as to what the phrase "textbased negotiations" means and what it specifically implies. He argues that the phrase is common in international negotiations and suggests that the question by the EU was mainly used as a way to stall the process, in this case the discussion on the future work of the committee as well as the new mandate for the IGC outlining the subsequent work programme. For parties cautious of the practice of textbased negotiations, it is risky to openly and directly contest the concept behind the term and the implied procedure, as this could be viewed as a refusal to commit to progress in the IGC in the form of a legally binding instrument. As some members of the African Group have stressed in the course of negotiations, such a refusal could be the reason for some delegations to leave the negotiation table. It is thus more conducive for stalling parties to ask for clarification on what the proposed approach of "text-based negotiations" entails than to state an obvious direct opposition:

[27] Our comment in relation to the term text-based negotiations in paragraph $2^{154}$ was more specifically around the fact that we asked questions of clarification yesterday afternoon in relation to what is meant by that term. And that pending a response to our question in relation to that we like the term to be bracketed and that pending a response

\footnotetext{
152 This is one reason for the "micro-editing" of texts (Groth 2010a). Stalling participants edit decisions about future work to keep the balance between showing a willingness to advance negotiations and not committing to too much.

${ }^{153}$ Interpreted statement on WIPO/GRTKF/IC/14/8 Rev. by the delegation of Angola, 03.07.2009. ${ }^{154}$ WIPO/GRTKF/IC/14/8 Rev., para. 2: “The Committee will undertake during the next budgetary biennium (2010/2011) text-based negotiations on genetic resources, traditional knowledge and traditional cultural expressions."
} 
we would also like to propose an alternative wording, so we are not proposing that it be deleted at this stage. ${ }^{155}$

In response to the delegate of Zimbabwe, the representative of New Zealand despite the open and direct accusation that they are only "pretending not to understand the term" - continues to ask for clarification of the term "text-based negotiations". This is possible because stalling delegations can argue that the explanations given as to what the term implies were not sufficient.

Another statement by the representative from Zimbabwe went even further, talking about "usual tricks" and the "questioning of simple terms and concepts". According to him, "even UN standard terms have been questioned, so maybe next time we should use a dictionary". He concluded with the analogy that he had "realized that the mother can not take care of its child", and that thus speeding up delegations could pursue the issues outside the IGC. However, his statement remained unanswered, and stalling delegations continued to ask for clarification and eventually introduced an alternative phrase. IGC negotiations are slow. Especially in the plenary session, in most cases there is no direct communication, meaning there is no immediate response by an addressee to a speaker's question. Even though a number of African Group delegations already explained the intentions and implications of WIPO/GRTKF/IC/14/8 Rev. and especially the approach behind text-based negotiations openly and directly, it is nonetheless possible, meaning not sanctioned, to ask for clarification and express reservations about a term's vagueness. Participants arguing for the inclusion of the phrase in most cases cannot respond directly to these reservations, as there is a list of speakers managed by the chair of the meeting. Hence it is "safer" for stalling parties to contest a phrase as being too vague or ambiguous - denying the possibility that it is a typical expression - than to openly state that one does not want to proceed to new and advanced stage of negotiations. Directly denying one's willingness to further the negotiation process would be unfavorable for a variety of reasons.

The reason why such indirection with regard to phrases like "text-based negotiations" is chosen is that even stalling delegations are interested in the continued stability of the committee, and one can assume they are aware of the implications and indexical meaning of the phrase to mean drafting text that will be the foundation for an international treaty or convention. It is, however, not in their interest to work toward a situation where a decision on adopting a text is imminent and their rejection of such a text would have to be made explicit. ${ }^{156}$ Indirect communicative

155 Statement on WIPO/GRTKF/IC/14/8 Rev. by the delegation of New Zealand at IGC 14, 01.07.2009.

156 The IGC's mandate for the 2010/2011 biennium called for a diplomatic conference at the end of the biennium that was to decide on such a text. Presumably, a conference would include a vote on a legal instrument where delegations would have to consent or dissent, thus making their position explicit. 
acts - such as asking to clarify a common term instead of overtly dissenting with the practice of text-based negotiations - are ways to maintain the committee's stability, even if this stability only entails that member states do not walk away from the negotiation table and continue the discussions. In this sense, maintaining stability is also a strategy of containment, limiting the discussions about the relation between GRTKF issues and intellectual property to the IGC and keeping them out of other fora like the WTO.

Another aspect of indirection in international negotiations is what can be termed the saturation of the negotiation process. Here, stalling actors doubt that enough information has been gathered or that enough analytic work has been done, and the opposite is true of speeding up delegations. By questioning the saturation of the process, the basic goals of the committee can be affirmed while the actual process is stalled. Both aspects - the construction of stability and the construction of saturation - will be illustrated in the following.

Regarding the aspect of stability, sustaining the "discursive illusion" of a shared venture to do good and protect cultural property from misappropriation is achieved by pragmatic strategies that contest denotationality. These strategies thus also contest a shared understanding of certain phrases that are inopportune to one's interests and goals. If an utterance has an undesired effect, the speaker can deny the prescribed meaning, and ask for clarification as to the specific implications. This entails a construction of the speech community both as a rather unstable entity with contesting denotations and presuppositions and a cohesive group of political actors all ascribing to universalistic values relating to cultural property. The basic motivations, principles and goals of the committee are reaffirmed, yet at the same time more specific aspects of communicative events are being displayed as vague and in need of clarification. This is a common practice in political contestation, and pragmatic terms like "text-based negotiations" (as a mode of negotiation) are just one example of such simultaneity of stability of ideology and instability of practice.

Showing a commitment to the core issues of negotiations can be accomplished using abstract wording as the following proposal by the European Community from IGC 14 shows:

[28] The IGC reaffirms its belief that traditional knowledge and other tradition-based creations, such as traditional cultural expressions, have taken on new economic and cultural significance within a globalized information society and that genetic resources have assumed increasing economic, scientific and commercial value to a wide range of stakeholders with the emergence of modern biotechnologies. The IGC acknowledges the intrinsic value of these issues which are also important in global trade and economic development. Indigenous and local communities have the right to maintain, control, protect 
and develop their intellectual property over such cultural heritage, traditional knowledge, and traditional cultural heritage. In this respect, the IGC will prepare a declaration on the value of traditional knowledge, traditional cultural expressions and genetic resources and their protection against misappropriation to be adopted by the WIPO General Assembly. 157

The broader ideational frame of reference for negotiations on cultural property entails that indigenous and local communities should have the rights to control, protect and develop their intellectual property, which is reflected in their cultural knowledge and expressions. This frame of reference implies an intrinsic value of cultural resources and speaks against their misappropriation. This connects the community of states and NGOs in international processes and creates the image of shared interests and motivations. Yet, this frame of reference has an only weak indexical meaning, meaning the specific pragmatic consequences of such generalized statements and sentiments are few. In the specific situation of [28], tabling such a proposal was a possibility to foil a much stronger proposal that included more concrete and binding wording as referred to in [25], [26] and [27]. The European proposal accordingly goes on to propose

[29] outcome oriented deliberations on genetic resources, traditional knowledge and traditional cultural expressions, based on their impartial treatment and noting the different levels of development in the texts.

While one frame of reference with weak indexical meaning - here, the allegedly shared presupposition that the committee has to act in favor of ILCs - is affirmed, another frame of reference with stronger indexical meaning is contested. An agreement on "text-based negotiations" as a mode of work would entail more specific mechanisms ${ }^{158}$ than, for example, "outcome oriented deliberations". The former would effectively speed up the proceedings considerably, whereas the latter would mean a continuation of non-committal negotiations.

\footnotetext{
157 WIPO/GRTKF/IC/14/11, Elements for the new mandate - Proposal by the European Community and its member states presented at IGC 14 , my italics.

158 IGC 14 in 2009 failed to compromise on a decision on future work ("The Committee did not reach an agreement on this agenda item", WIPO/GRTKF/IC/14 Decisions), largely due to the fact that Group B was not willing to accept the term "text-based negotiation". WIPO's GA later that year, however, decided to renew the IGC's mandate for the 2010-2011 biennium in favor of wording proposed by the African Group at IGC 14 (WIPO/GRTKF/IC/14/8 Rev.): "The Committee will, during the next budgetary biennium (2010/2011), and without prejudice to the work pursued in other fora, continue its work and undertake text-based negotiations with the objective of reaching agreement on a text of an international legal instrument (or instruments) which will ensure the effective protection of GRs, TK and TCEs" (WIPO/GRTKF/IC/15/REF-DECISION 28).
} 


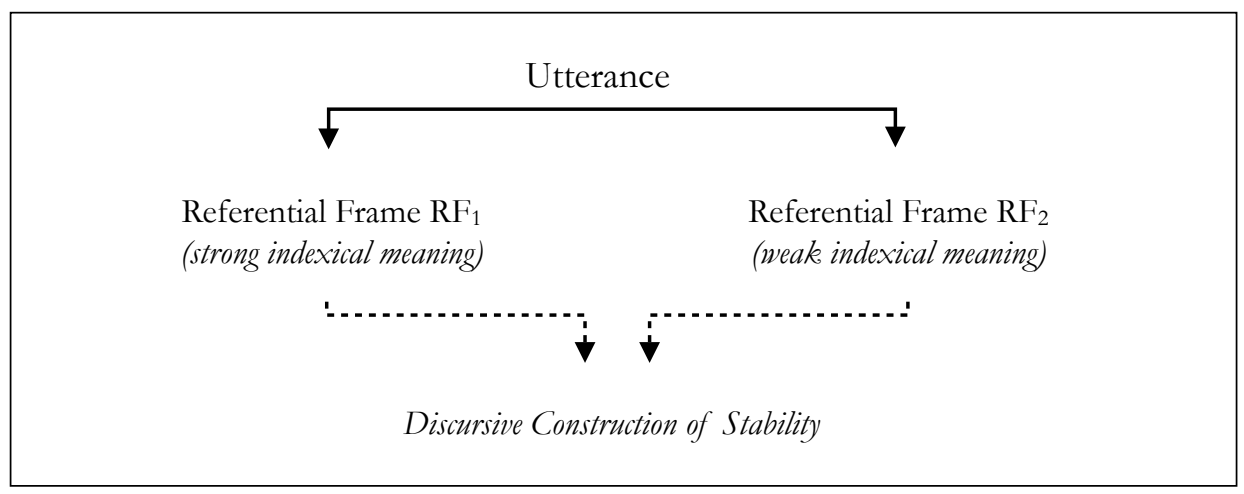

Figure 7. Multiple Referential Frames for the Construction of Stability

Thus, two types of referential frames can be distinguished for communicative events like this (Fig. 7). While the first $\left(\mathrm{RF}_{1}\right)$ has strong indexical meaning and entails the proposal of specific actions as well as pragmatic markers, the second referential frame $\left(\mathrm{RF}_{2}\right)$ has only weak indexical meaning, few to no pragmatic implications and mainly generalized statements.

In the discussions on "text-based negotiations", $\mathrm{RF}_{1}$ refers to the assumption of a high degree of shared denotationality as to specific terms which signify different stages of negotiation and their implications. In $\mathrm{RF}_{1}$, the conventional nature of the terms as well as their implications are established, constructing the image of a natural and common sequence of negotiation phases. This frame is partly exclusive as it points out the conventionality of denotations and indexical meanings by excluding contesting meanings. The stability of the speech community in this case is constructed by pointing to semantic and indexical patterns that have emerged over the course of time in various fora, shared by opposing actors as well. Using $\mathrm{RF}_{1}$ is a very direct way to signal and invoke frames with rules and obligations that require specific actions.

Using $\mathrm{RF}_{1}$ invokes certain rules and obligations for the communicative event. In the case of "text-based negotiations", one of those actions required would be the drafting of text as a basis for an international legal instrument, and thus a different stage of negotiations than exchanging views or creating gap analyses. This presupposes and at the same time discursively reproduces a high degree of stability of the speech community as it only makes sense to move forward in negotiations if there is common ground and a shared understanding about what the problems are and what the solutions might be. The process of gathering information during fact-finding missions, questionnaires, professional expertise and extended discussions in the IGC is thus the groundwork for this advanced stage that delegations 
eager to speed the proceedings can refer to. ${ }^{159}$ Invoking this referential frame at an earlier point of time would have been counterproductive as the parties in opposition would have pointed out the lack of information and discussion, and it does thus presuppose a certain degree of stability in terms of prior work and documentation. Requesting to move on to text-based negotiations is therefore also the attempt to discursively reproduce already given stability: if opposing delegations agree to this step, they also admit to a certain degree of stability in the form of already achieved work.

The "discursive illusion" of a shared venture to find ways to protect cultural property is vital for stalling parties as well. For them, proposals like WIPO/ GRTKF/IC/14/8 Rev. pose the problem that the invocation of $\mathrm{RF}_{1}$ (as well as the corresponding rules and obligations) by speeding up delegations requires maintaining a constructed stability while not being able to concede to the proposed new mode of negotiation. Openly contesting such proposals would result in an instability that could be blamed on the refusing party: simply rejecting "text-based negotiations" as an advanced stage of negotiations could be portrayed as a rejection of the whole process. In such cases, switching to the second frame of reference $\left(\mathrm{RF}_{2}\right)$ is a possibility to maintain stability while not making substantial concessions. ${ }^{160}$ $\mathrm{RF}_{2}$ in this case has weaker indexical meaning and its implications are - compared to $\mathrm{RF}_{1}$ - only minor (cf. [28] and [29]). A generalized affirmation of shared basic values - see example [28] - is also a strategy to maintain the image of shared interest, thus reaffirming the grounds on which negotiations take place, without necessarily achieving substantive progress and committing to a schedule of future work. Shifting to a different referential frame with less specific indexical meaning $\left(\mathrm{RF}_{2}\right)$ on the surface achieves the same result, in this case the discursive construction of stability, as a reference to a more pragmatic referential frame $\left(\mathrm{RF}_{1}\right)$.

Nonetheless, even if the result is the same, the function that a given referential frame realizes is a different one. $\mathrm{RF}_{1}$ is invoked to build pressure on the opposing parties. In the case of a refusal to agree, the stalling actors can be blamed directly for obstructing the negotiation process, or more strongly, for neglecting and violating universal human or cultural rights. The claims made in $\mathrm{RF}_{1}$ are portrayed as the ideal solution, one without any alternatives. $\mathrm{RF}_{2}$, on the other hand, has the compensatory function to divert blame and to stabilize the illusion of a shared venture while expressing "technical" objections. The claims made in $\mathrm{RF}_{1}$ are refuted in so far as they go beyond abstract motivations, and pragmatic stalling mechanisms

\footnotetext{
${ }^{159}$ However, it has to be noted that these factual documents are not discussed very often, at least not in detail. They are referred to from time to time, but their content or the arguments they contain are not made explicit.

${ }^{160}$ It should be noted that switching referential frames is different from switching code. The metapragmatic awareness involved in the strategic and conscious usage of such a pragmatic strategy is one major difference between code and frame switching. Another specificity of such switching of referential frames is that it does not necessarily involve or require code switching.
} 
such as filibustering, calling for more fact-finding and discussion, and so forth are used to endorse these abstract values, thus creating stability while not conceding to the demands of opposing delegations.

The basic functions of references to $\mathrm{RF}_{2}$ are to stall the proceedings while maintaining the illusion that one wants to speed up the proceedings and find a solution for the problems at hand. Openly stalling, stating that one is not interested in any progress during the main session and that one only participates in the IGC in order to keep the issues from being discussed in other fora, would inevitably lead to conflicts and instability that could cause the IGC negotiations to come to an end without any results. As this is not in the interest of Group B or other stalling parties, stalling mechanisms have to be "veiled" or compensated for by maintaining the possibility of a consensual decision that would benefit parties interested in implementing an international legally-binding agreement on intellectual property and TK, TCEs, and GR. The stability of the process needs to be maintained. In this case, keeping the process stable and the participants of the speech community at the negotiation table does not mean only to maintain the status quo. Rather, it includes the need to move forward and to achieve progress in negotiations. ${ }^{161}$ An example for this is the following statement by the United States of America at IGC 14:

[30] During the next day or two, we are interested in learning more about the proposal from its proponents, we are interested in learning more about the view of other delegations, and learning more about the financial and administrative implications of the proposal from the secretariat. $^{162}$

Embedded in other statements that reaffirm the shared goals and motivations of the IGC, utterances like this unite different pragmatic functions. On the one hand, they establish the argumentative groundwork for keeping negotiations going without making to many concessions by asking for extended clarifications on items such as "financial and administrative implications" or by asking for a continuation of discussions rather than proceeding to the next stage of negotiations. On the other hand, they express an interest in moving forward by "learning more" about the issues under negotiation, thus creating the foundation on which a convention could be drafted.

The combination of these two functions bypasses the highly indexical referential frame $\mathrm{RF}_{1}$ and at the same time contributes to the discursive construction of stability. No specific commitments or concessions are being made, and yet the in-

\footnotetext{
161 A basic mechanism for this is "micro-editing" written decisions on future work or the drafting of text.

162 Opening statement by the delegation of the United States of America at IGC 14, 29.06.2009.
} 
vocation of $\mathrm{RF}_{2}$ still entails an abstract affirmation of the process. Stalling parties using a referential frame, with its abstract affirmative value and low indexical meaning can, if pressed, argue they too want the process to continue. The functional difference is that their construction of stability proposes a vague and slow process rather than specific steps that would speed up the proceedings. Both include some sort of moving forward, they differ in the degree.

This invocation as well as the shifting of referential frames is contingent on context. Shifting frames presupposes realizing several contextual variables, for without them, shifting as a pragmatic strategy may not be conducive to one's interests. The invocation of $\mathrm{RF}_{1}$ requires a certain amount of saturation, i.e. whether enough work to be built on has been already done, or progress in negotiations. If this condition is not met, the indexical meaning of $\mathrm{RF}_{1}$ can easily be countered by referring to the incipient nature of the process and how premature the proposed measures are. In the case of the IGC, this saturation concerns among other things the availability of various texts. ${ }^{163}$ Furthermore, the expression of national positions, the inclusion of comments by ILCs, NGOs and related IGOs during the IGC process as documented in the extensive meeting reports can be considered to be a prerequisite for the invocation of a referential frame that calls for advanced work or the drafting of a treaty or convention.

The question whether the degree of saturation is sufficient is, of course, subject to negotiation. The ability to refer to $\mathrm{RF}_{1}$ is thus contingent upon the ability of actors to agree the work done to date is sufficient to build upon. The construction of saturation differs from the construction of stability in that the latter construes the IGC as a speech community with shared interests and denotations, while the former denotes a specific stage within the communicative activities that looks back at what has or has not been achieved. There is a simultaneity of stability and instability at different levels, the most fundamental being the perceived or actual commonality of abstract values associated with human rights, cultural rights, indigenous rights, and authors' rights.

\footnotetext{
163 Among those texts are the compilation of gap analyses on the protection of TCEs/EoF and on the protection of TK (WIPO/GRTKF/13/4(b) Rev. and WIPO/GRTKF/13/5(b) Rev.), questionnaires on national experiences with the legal protection of TCEs (WIPO/GRTKF/IC/2/7) and on existing forms of IP protection for TK (WIPO/GRTKF/IC/2/5 - see http://www.wipo.int/tk/en/ consultations/archive.html, accessed 27 April 2012, for a list of questionnaires and comments gathered during the IGC process), case studies and fact-finding reports (See http://www.wipo.int/tk/en/ resources/ (accessed 27. April 2012) for an overview of case studies and reports) as well as draft provisions and articles (WIPO/GRTKF/IC/18/4 on TCEs/EoF, WIPO/GRTKF/IC/18/5 and WIPO/GRTKF/18/7 on TK). The case study edited by Terry Janke (2003) and the fact-finding missions carried out in 1998 and 1999 (see http://www.wipo.int/tk/en/tk/ffm/report/index.html. accessed 27. April 2012) should be noted in particular.
} 
On the basis of these values and by way of pragmatic strategies, stalling and speeding actors IGC actors can reaffirm their commitment to the process. Openly dismissing or neglecting these values would not benefit their interests, and could have repercussions in diplomatic or civil society discourses.

There is also the matter of a shared understanding of terms and issues. These are quite specific concepts and their implications, such as the meaning of "textbased negotiations" or what "traditional knowledge" is. While stability can exist at the underlying level of generalized affirmation of the IGC process, the level of shared meaning can be unstable. Yet, by referring to the stable level of generalized affirmation as well as taking a historical perspective at the practical level, the stability of one level can be mapped onto the other. By making reference to preceding work and texts, shared values are projected onto the level of shared understanding. As a result, stability is reproduced in the process of moving forward by looking at past work and perceived shared values.

The construction of saturation therefore bolsters the argument of speeding up parties by connecting these different levels. Stalling or opposing parties do not have to target the layer of stability related to the generalized affirmation of the IGC's objectives, but can concentrate both on the construction of saturation and on the construction of the stability of shared understanding. If the committee has not done enough work, meaning not collected and analyzed enough data to make an informed decision, and if, therefore, participants do not share an understanding of basic terminology and concepts, then moving forward does not make sense, regardless of the degree of stability on the level of generalized affirmation.

The discussions about the definitions of TK and TCEs during the twelfth session of the IGC in early 2008 are a prime example for this discursive construction of saturation. The main positions during these discussions were that definitions of TK and TCEs are a prerequisite for moving forward in negotiating an agreement (bottom-up approach), and that there was sufficient shared understanding of what TK and TCEs are to proceed with broad working definitions and refine them during the course of negotiations (top-down approach). As summarized in this statement by Pakistan from the report of IGC 12

[31] [t]here were two options. The first option was a bottom-up approach that was to continue these discussions, see more and more clarity and develop consensus on these issues. This approach might help to improve the comfort level of different countries, but it would be long and that too without any surety to see light at the end of the tunnel. The second option was a top-down approach that was to take a decision to start discussions on any international instrument. Pakistan understood that a majority of countries was in favor of this approach, whereas others felt that time was yet not ripe for such discus- 
sions. Taking this approach was neither new to the UN system nor to the WIPO itself, as was done in case of a Broadcasting Treaty. ${ }^{164}$

The communicated fault-lines in this discussion were thus whether there were or were not enough data or consensus on the ground of which it was possible to advance negotiations. Various countries of Group B generally considered to be stalling WIPO IGC negotiations - for example Canada, Japan, the United States of America, and the European Union - voiced their concerns that there was "a lack of concurrence and consensus" about the essence of TK and TCEs. ${ }^{165}$ Their line of argument was that a "common understanding was needed" for clear definitions of TK and TCEs, and that the "depth of understanding" concerning terms and perceptions achieved so far was "insufficient for a decision on the international level." (ibid.) Due to these claimed insufficiencies, for those countries drafting a formal definition was not considered to be a viable option, and furthermore all of the IGC's work was portrayed as having been fruitful, but not sufficient, as this statement by the Canadian delegation shows:

[32] A great deal of very useful work had been accomplished by the Committee to date. The issues at stake were complex and extremely challenging and further discussions were required to identify what concrete steps could be taken with regard to the IP-related aspects of TCEs and TK. Canada was committed to deepening the discussions with a view of reaching a common understanding of these multidimensional subject matters, bearing in mind the considerable amount of work Member States and observers had undertaken in refining the draft policy objectives and general guiding principles on TCEs and TK. [...] Canada looked forward to engaging constructively in discussions, making progress this week on all core agenda items, and working towards defining a practical and concrete path forward for the work to be undertaken by the Committee for the present biennium. ${ }^{166}$

Note that there is on the one hand a generalized appreciation of the IGC's previous work that is bolstered by a description of the subject matter as "complex and extremely challenging", and on the other hand a specific reference to problems

\footnotetext{
164 WIPO/GRTKF/IC/12/9:21. Analogously to establishing the conventionality of a term or a phrase (see example [03] - "text-based negotiations"), delegations wanting to speed negotiations up can try to establish that a procedure is conventional, as with the reference to the UN system in general and to a specific WIPO treaty here. Such references are used frequently in IGC negotiations to portray specific approaches as inherent to and common within UN structures, rather than as procedural anomalies (author's notes from February 2008, October 2008, June 2009, and December 2010). This can be termed a "contestation of conventionality" and builds on contextual contingencies.

165 Author's notes, IGC 12

$166 \mathrm{WIPO} / \mathrm{GRTKF} / \mathrm{IC} / 12 / 9: 17$.
} 
that one is not able to solve due to external circumstances, and not due to a lack of willingness. According to this conceptualization, the reason why Canada and other Group B countries oppose formal definitions is not because they do not want them, but because there are technical and structural impediments that make them objectively impracticable:

[33] At the most fundamental, the factual extraction demonstrated that there was as of yet no consensus as to a definition of TCEs. It also showed that there continued to be a lack of concurrence as to the scope of other terms such as "community" and "beneficiaries". As noted in the 1998-1999 WIPO Fact-Finding Mission (at p. 211), "Lack of terminological clarity can confuse and obscure what is already, terminology aside, a complex inquiry." Canada supported engaging in focused, in-depth analysis of these fundamental issues in order to achieve greater consensus and to move the work of the Committee forward. [...] It was hoped that the Committee would continue to engage in an in-depth, step by step analysis of these important over-arching issues in order to achieve greater understanding of the definition of "TCEs", "communities" and "beneficiaries". This would help Member States in reaching further consensus on the matters before the Committee. ${ }^{167}$

This combination of positive acknowledgement of previous work, articulation of eagerness to deepen a "common understanding", and pointing to substantial and procedural insufficiencies is a common pattern in IGC negotiations. It is used especially in the so-called "opening statements" (see above). Similarly, this statement by the delegation of the United States of America can be read as an attempt to legitimate the declination of formal definitions:

[34] The Delegation of the United States of America thanked the Secretariat for its work on the preparation of the "factual extraction" on TCEs, which captured the richness and diversity of the views of the Committee and which should prove to be an invaluable tool as the Committee moved forward to deepen its discussion of these complex questions, with a view toward gaining a shared understanding of these difficult issues. The record of work of the Committee was indeed extensive. In the view of the Delegation, however, the extensiveness of the record of the discussions, along with the extremely useful documents and studies produced by the Secretariat, was not a cause for alarm or apology, and certainly not a reason to depart from the tradition, within this Committee, of Member State-driven delib-

167 WIPO/GRTKF/IC/12/9:39. 
erations. Rather, the rich record of work, not unlike a traditional tapestry, reflected the differing views and underlying values of the Member States that had produced it. The Delegation would view with considerable skepticism any attempt to substitute the actual views of Member States with distillation of those views in a document produced by the Secretariat. ${ }^{168}$

Again, what can be observed from this statement from the report of IGC 12 is that an abstract commitment to the process coupled with a high evaluation of the progress the committee has achieved so far (cf. [30]) is "countered" by the regret that due to the complexities of the subject matter and the diverse points of view, a formalization of this progress (for example in the form of definitions of TCEs and TK) cannot be attained. This process can be described as the argued causality in the shifting of referential frames: because - so the argument goes - there is not enough saturation in negotiations, one has to switch from specific methods of formalization like definitions $-\mathrm{RF}_{1}-$ to an unspecific mode of work without immediate outcomes $-\mathrm{RF}_{2}$. The decision to do so can thus be portrayed as the result of procedural and technical conditions, rather than as the result of specific constellations of interest.

In the discussions about a top-down or a bottom-up approach for definitions of TK and TCEs, speeding countries referred to the set of "factual extractions"169 prepared by the WIPO secretariat for IGC 12 as a valuable resource and a point of reference for drafting definitions. Proponents of this top-down approach (the African Group, India, Brazil) voiced their demand for formalized working definitions. This formalization would have entailed a written definition which IGC member states would need to agree about. These definitions would have - and that was one of the concerns of stalling delegations - established a precedent and formal point of reference for all subsequent negotiations. The formulations could easily have taken on the character of "typical expressions" and thereby restricted the room for maneuver of delegations not interested in progress of IGC negotiations.

The general argument of those delegations in favor of formalized working definitions was that they "are not complete, but sufficient as a base for progress". ${ }^{170}$ In their view, focusing on a working definition would allow the IGC to move ahead and achieve progress. The definitions would not need to be perfect, as they could be revised at a later point of time.

The problems of definitions - their static nature, vis-à-vis a dynamic object as well as a multitude of differing national, regional, and cultural specifics - were acknowledged by various delegations. Yet countries such as India, Brazil, or Algeria

\footnotetext{
168 WIPO/GRTKF/IC/12/9:37.

${ }^{169} \mathrm{WIPO} / \mathrm{GRTKF} / \mathrm{IC} / 12 / 4$ (b) for TCEs and WIPO/GRTKF/IC/12/5(b) for TK.

${ }^{170}$ Statement of the American Folklore Society at IGC 12, 26 February 2008.
} 
argued that to make headway, drafting a working definition was a prerequisite. It could be accomplished with relative ease by taking the IGC's "Revised Objectives and Principles" for TK and TCEs as a point of reference. ${ }^{171}$ Such documents drafted by the WIPO secretariat had distilled "in coherent and focused form the kind of specific questions that may need to be weighed by policymakers at the national, regional and international level, when considering the appropriate form and means of protection of TCEs/EoF" 172 and TK. "Without prejudging [their] status or legal implications", these texts included working definitions of TK and TCEs. For TCEs this definition read:

[35] TCEs/EoF include tangible and intangible forms in which traditional culture and knowledge are expressed, communicated, appear or are manifested. They may be verbal expressions or symbols, musical expressions, expressions by action, such as dances and other performances, and tangible expressions, such as productions of art, in particular, drawings, designs, paintings (including body-painting), carvings, sculptures, pottery, terracotta, mosaic, woodwork, metalware, jewelry, baskets, needlework, textiles, glassware, carpets, costumes; handicrafts; musical instruments; and architectural forms. [...] To be eligible for specific protection, TCEs/EoF would need to meet three criteria. They should be: the products of creative intellectual activity, including individual and communal creativity; characteristic of a community's cultural and social identity and cultural heritage; and, maintained, used or developed by such community, or by individuals having the right or responsibility to do so in accordance with the customary law and practices of that community. ${ }^{173}$

For TK the proposed definition was:

[36] TK is defined in general, indicative terms as the content or substance of knowledge resulting from intellectual activity in a traditional context, and is not limited to any specific field, extending to agricultural, environmental and medicinal knowledge, and knowledge associated

\footnotetext{
171 These documents were originally drafted for the IGC's seventh session in November 2004 and have been reproduced for IGC 9 in April 2006 after a first review process; they have been reproduced for IGC 12 as WIPO/GRTKF/IC/12/4(c) for TCEs and WIPO/GRTKF/IC/12/5(c) for TK. They were complemented both with an overview over the current work on TCEs (WIPO/GRTKF/ IC/12/4(a)) and TK (WIPO/GRTKF/IC/12/5(a)) prepared by the secretariat as well as with two sets of "factual extractions" (WIPO/GRTKF/IC/12/4(b) and (WIPO/GRTKF/IC/12/5(b)) including comments from delegations and observers on the original documents from IGC 7 and pointing to points of convergence and differences between the positions of WIPO member states.

$172 \mathrm{WIPO} / \mathrm{GRTKF} / \mathrm{IC} / 12 / 4(\mathrm{c}): 4$.

${ }^{173} \mathrm{WIPO} / \mathrm{GRTKF} / \mathrm{IC} / 12 / 4(\mathrm{c}): 7$.
} 
with genetic resources. [...] However, the draft suggests that to be eligible for specific protection against misuse or misappropriation, more precision is needed, and that TK should (i) exist in a traditional and intergenerational context; (ii) be distinctively associated with a traditional or indigenous community or people which preserves and transmits it between generations; and (iii) be integral to the cultural identity of an indigenous or traditional community or people which is recognized as holding the knowledge through a form of custodianship, guardianship, collective ownership or cultural responsibility. This relationship may be expressed formally or informally by customary or traditional practices, protocols or laws. ${ }^{174}$

In the view of speeding up parties, this was an adequate basis for the committee to move on to the substantive issues that needed to be resolved. The delegate from Nigeria urged the committee to establish "clear guidelines for work", 175 and that there had to be more results than just an exchange of statements. ${ }^{176}$ At IGC 13, the African Group tabled a proposal on the "Protection of Traditional Knowledge, Traditional Cultural Expressions and Genetic Resources"177 that collected the views of delegations on ten central issues discussed in the IGC. ${ }^{178}$ It included as issues the "Definition of Traditional Cultural Expressions that should be protected" and the "Definition of Traditional Knowledge".

The proposal for the definition of TK (see Fig. 8) states that

[37] [t] he general consensus is that a working definition of Traditional Knowledge (TK) is important. The views vary from a broad definition to a more concise and narrower definition.

It continues by listing three views on the issue of definition said to be extracted from various documents and discussions of the IGC. By outlining these views as well the separate aspects they contain and by framing them as compatible with each other after minor adjustments (e.g. by phrasing the third view in a way that can be complemented by the "way forward"), the proposal implies that there is enough information available and therefore enough saturation. The differences

\footnotetext{
174 WIPO/GRTKF/IC/12/5(c):7.

175 Author's notes from IGC 12.

176 This exchange about a top-down or bottom-up approach to definitions on TK and TCEs was part of the opening statements of IGC 12 that lasted until the late afternoon of the third day of this session (see the beginning of this chapter for a more detailed view on these opening statements).

177 WIPO/GRTKF/IC/13/9.

178 The number and content of the issues refers back to a list of issues that was prepared by the secretariat following a decision at IGC 10. These lists on TK and TCEs are reproduced as annex I in the report of IGC 11 (WIPO/GRTKF/IC/11/15).
} 


\section{WIPO/GRTKF/IC/13/9}

Annex, page 2

\section{THE PROTECTION OF TRADITIONAL KNOWLEDGE}

\section{Issue 1 - Definition of Traditional Knowledge}

The general consensus is that a working definition of Traditional Knowledge (TK) is important. The views vary from a broad definition to a more concise and narrower definition. Article 3 ("general scope of subject matter") in document WIPO/GRTKF/IC/11/5C would be a good starting pointing for discussions.

View 1:

The definition should:

- Include "knowledge systems, generated from local indigenous or traditional communities" as well those "generated, preserved and transmitted in different approaches, different people, including ethical (minorities) groups".

- "Be anthropological"

- $\quad$ "Be accumulated knowledge that was the result of intellectual activity and insight into a traditional context and included the know-how, skills, innovations ... embodied in the traditional lifestyle systems".

View 2:

Questions whether the definition be formal or rigid? The definition should consider the evolving nature of TK.

View 3:

Precise definition important in order for a common understanding.

Definition required to obtain legal certainty so that it's clearly identifiable and

described. A single definition is not appropriate.

- Look at the purposes of protection, whether that protection is by legal, non-legal, national and international means.

Way Forward:

The IGC should have a concise and flexible working definition which is complemented by a list of examples of TK.

The definition of TK should also include innovative aspects. The term should not be limited to a single technical field and be inclusive of all knowledge systems.

Figure 8. African Group Proposal on the Protection of GRTKF (WIPO/GRTKF/IC/13/9).

between speeding up and stalling, according to this perspective, can be resolved by the outlined proposal. As it would take into account all difficulties, a top-down approach was for that reason portrayed as being suitable for the concerns voiced 
by Group B countries in favor of a bottom-up approach. On the terminological level, speeding up delegations argued that the available documentation and expertise are sufficient for formulating a working definition as a step forward. Stalling delegations, on the other hand, argued there was neither enough clarity nor sufficient consensus on substantive questions, such as over the elements of a definition of TK and TCEs.

It is important to note this difference between the conventionality of procedure (as in discussions about text-based negotiations) and the conventionality of terminology (as in discussions of top-down versus bottom-up approaches to defining TK and TCEs). They relate to different pragmatic dimensions. The first asks whether a procedural approach (denoted by a term) is common and whether there is a shared understanding of the factors involved in a specific approach. By focusing on procedure, stalling delegations can bypass substantive questions, for example about the specific contents of a text as the basis for negotiations. The arguments against text-based negotiations are thus not that there is a lack of shared understanding as to the importance of an instrument for the protection of TK and TCEs, but that the proposed procedure is unclear. On the other hand, the discussions on the way to move forward with regard to definitions primarily focus on substantive debates, arguments and views denoted by a term. "Text-based negotiations" thus point to procedural issues, "traditional knowledge" to substantive discussions. In both cases, stalling delegations can, by employing a contextualized instantiation of a communicative strategy, use an indexically weak referential frame. They can either argue that the procedure is not common and needs to be explained, or they can argue that the available documentation and information is not sufficient to make decisions about substantive questions.

In certain situations, references to $\mathrm{RF}_{2}$ do not have the ability to maintain the "illusion of stability" that is mediated by the construction of shared goals and motivations. There are situations where invoking abstract motivations, to avoid highly indexical speech, is not enough and concessions have to be made. In such instances, an imbalance between a high level of assumed stability, meaning a high degree of shared motivations, openly expressed, and a contested degree of saturation, meaning divergent views on the quality and sufficiency of work carried out by the committee so far, can be observed. Assertions of commitment are not sufficient, and a frame of reference of generalized affirmation cannot compensate for the lack of progress on a pragmatic level.

Such situations are usually enunciated by speeding up delegations. At IGC 14, the discussions about the new mandate lead to a number of delegates openly criticizing stalling delegations, with the speaker of the African Group complaining that "all we do is talk, talk, talk", the Peruvian delegate describing the session as the "same chit-chatting as always", the Egyptian delegate comparing the IGC to a "talk-shop", or the delegate from South Africa arguing that it was mostly "talking for the sake of talking". The delegate from Angola in a very figurative statement 
used the metaphor of the IGC as an "aircraft with no destination", and the Brazilian diplomat described the approach taken by stalling delegations as "socratic" in that "it does not seek results, but wisdom". Yet, he argued that "we need courage now to take hard decisions" and not "act like philosophers". ${ }^{179}$

These examples illustrate the contextual dependency of referential frames. They were all uttered in a rather intense moment of IGC negotiations: IGC 14 was the last meeting before WIPO's General Assembly would decide on the committee's new mandate. Taking a decision to move forward was thus viewed by many delegates as essential. Without an agreement on the new mandate, the discussions would have to be continued in the General Assembly, with an unclear outcome. By referring to this situation, speeding up delegations were able to dismiss $\mathrm{RF}_{2}$ as insufficient and to establish $\mathrm{RF}_{1}$ as the only way forward to ensure the future existence of the committee.

The effectiveness and potency of shifting frames is thus connected to specific situations that link pragmatic strategies, referential frames, and the socially perceived and mediated positions in negotiations: there is a dialectical relationship between different desires and restrictions to these desires at a distinct moment that creates the possibility of the realization of a strategy. These desires and restrictions are differentiated among actors or groups of actors, either as the speakers of utterances or only as receivers and observers of communicative events. For the IGC as a speech community - a dynamically constituted group of social actors from various backgrounds - this means that at any given point in negotiations, there is more than one "frame" and thus also multiple sets of desires and restrictions.

The success of invoking referential frames as a pragmatic function with strong $\left(\mathrm{RF}_{1}\right)$ or weak $\left(\mathrm{RF}_{2}\right)$ indexical meaning is contingent on "targeting" powerful sets, i.e. framing one's interest or strategy in a way that is convincing to groups of actors that matter. As outlined above, this concerns both questions of terminology and questions of procedure. This "twofold nature" of saturation creates ambiguity and thus a lot of leeway for both stalling and speeding delegations. If one of the two aspects is covered, e.g. if there is a consensual definition of property rights (to take one of the terms that can seemingly be translated a lot easier than TK or TCEs), stalling actors can refer to a lack of procedural clarity, e.g. by pointing to the need to examine existing intellectual property legislation on national, regional, and international levels in order to achieve an overview about possible gaps (cf. the IGC's two gap analyses for TCEs and TK). The other way round, speeding delegations can point out procedural saturation, for instance long-drawn prior negotiations and existing working documents, in order to claim stability that allows for the drafting of definitions or of an agreement.

${ }^{179}$ Author's notes from IGC 14. 
The group of relevant social actors that matter for the invocation of frames or sets is constituted dynamically and differs from communicative event to communicative event. Civil society organizations can for example at times be important to build pressure while they are utterly neglected in the consultation of regional group coordinators. Similarly, ILCs are usually paid more attention at the beginning of an IGC meeting, while they are predominantly neglected at the end of a session where member states often seclude themselves to internal negotiations such as consultations between the chair and regional group coordinators. Even some member states become less relevant in the course of a meeting, as they either hold no stake in the negotiations or they simply have no interest in the substantive questions discussed. The instantiations of communicative patterns and strategies are for that reason contingent on the specific context constituted by, among other factors, relevant social actors that need to be addressed.

Referential frames thus correspond with and depend on specific groups of recipients as audiences that determine the suitability of referential frames. These groups are part of the specific context of an utterance. They are constructed in communicative events, and at the same time have a social materialization in the form of NGOs, ILCs, member states, and other observers and participants of the IGC process. Three main dimensions of these audiences can be distinguished: (1) their competence, including what the specific audience understands (or wants to understand) on what level. The various levels relate to semantic and pragmatic meaning, meta-pragmatic awareness, the ability to differentiate various functions of utterances and to weigh technical, legal and ethical implications. The second dimension is (2) time as the perceived and mediated sense of what is an adequate span of time after which decisions should be taken, and it is influence by external factors (such as domestic policy pressures) and internal factors. A third dimension consists of (3) the interests and motivations of actors as the various stakes of the specific audience. Referential frames are partly determined by relevant audiences and their dimensions have thus to be taken into consideration when analyzing negotiations. As concerns the question of blame for failed or stalled negotiations, the shifting of referential frames needs to take into account these different audiences as well as the dynamic relationship between referential frames: $\mathrm{RF}_{2}$ implies a shared understanding and interest that is reinforced by the involvement and appreciation of civil society and pressure stemming from other fora and institutions. These specific audiences require the acknowledgement of $\mathrm{RF}_{2}$ as an ideational foundation of negotiations. If stalling delegations would not use $\mathrm{RF}_{2}$ and basically dismiss the calls for a legal instrument, direct reproaches as well as a additional pressure by audiences interested in the protection of GRTKF would be the consequence. There is thus a "back and forth" between the expression of commitment and the dismissal of concrete procedure. However, this back and forth must move forward as the excuses to stall the procedures proposed by speeding delegations in $\mathrm{RF}_{1}$ get subsequently weaker: at some point of time, $\mathrm{RF}_{2}$ is overburdened and not able to 
compensate and create stability anymore. Then, concessions in $\mathrm{RF}_{1}$ need to be made (for example the introduction of inter-sessional working groups and textbased negotiations that was, in the last instance, agreed to by stalling delegations as well). However, such concessions are able to make $\mathrm{RF}_{2}$ viable again and to stall negotiations by dismiss demands in $\mathrm{RF}_{1}$. This does not necessarily mean that at some point, all $\mathrm{RF}_{1}$ demands are fulfilled as a result of the progression of concessions, as other compensatory mechanisms can be installed (for example bi-lateral agreements or national legislation). In conclusion the shifting of referential frames can be viewed as an instability that produces stability and instability at the same time, and therefore allows for pragmatic strategies as outlined above. It is a productive "dialectical movement" that lacks the capacity to relapse behind its thesis, as relapsing would imply a rejection of the process.

Silverstein introduces four categories of pragmatic strategies that are all based on such contingencies or instabilities of context, meaning a lack or incompleteness of shared pragmatic norms or contextualization (Silverstein 1976:47-48): Pragmatic contradiction is a strategy where "two or more communicative media" signal "contradictory indexical meanings", or "contradictory highly presupposing indexes within the same medium" are employed. Here, the speaker of an utterance simultaneously signals two meanings, as is common for the shifting of referential frames as outlined above where utterances signal a commitment to the process and a simultaneous rejection of the specific procedures suitable to achieve results. Residual semanticity refers to the semantico-referential meaning which "a speaker can claim after the fact for potentially highly pragmatically charged speech" (47). Similarly, stalling delegations can claim their reservations are caused by technical questions or a lack of understanding, and not by their interest in stalling.

The third strategy, imputed indexicality, denotes the strategy where "a speaker can create a social persona for himself, playing upon the hearer's perspective of imputed indexicality, where the speaker has characteristics attributed to him on the basis of the rules of use for certain utterance fractions" (47). As a way to veil controversial motivations, communicative strategies can contribute to the representation of a speaker as uncontroversial, for example by heavily stressing the presumably shared ideational foundations. Diplomatic non-indexicality as the fourth strategy (making an analogy with diplomatic non-recognition in foreign policy) "allows the hearer to respond to speech as though it constituted a semantico-referential event, all the while understanding completely the distinct function 2 of the indexes which overlap in surface form" (48). This last strategy is common in IGC negotiations, especially with regard to the cases where speeding up delegations overtly critique the stalling tactics. Responses to such utterances usually fail to materialize or only refer to the aspects of the utterances that can be reduced to their semantico-referential meaning. The four strategies that Silverstein outlines can thus be found in WIPO IGC negotiations. 
In all of these cases the ability to strategically employ language depends on a kind of uncertainty (or diversity) of context that allows for a pragmatic operation, be it signaling the affiliation to a certain social group, the denial of a certain intention, the coding of an event according to certain perspectives, or the invocation of specific frames. However, as instantiations of communicative patterns and strategies, these pragmatic strategies need to be examined in context of specific referential frames and audiences - otherwise, neither their function nor their indexical meaning in relation to their referential meaning can be elicited. An example for this contextualization concerns the question of metapragmatic awareness: who understands these strategies to the desired effect? It can only be answered by taking into account the broader context and history of the committee, cultural property negotiations, communicative patterns, referential frames and audiences. This concerns not only the analytical level, but mainly the level of participation, that is the specific communicative practices. The knowledge of context (and thus also of the participants partaking in a communicative event) is essential to choose a way conducive to mediating one's perspective on an event (for example to convince other participants of the advantages of one's strategy or position), to choose a coding scheme that fits bureaucratic systems, or to shift to a referential frame.

Yet, on the other hand this knowledge of context is also vital when analyzing such communicative events. In order to understand the perspectives taken, the coding schemes applied, or the referential frames invoked, it is important to distinguish between semantics, pragmatics and metapragmatics (by way of linguistic analyses) and to carry out the necessary contextualization. As Silverstein puts it, "the use of some particular word or expression at a moment in denotational texttime $[\ldots]$ comes differentially to invoke - to summon to the here-and-now - some specific cultural concept in a schema of such" (Silverstein 2004:634), and thus, an analysis of only text and talk would inevitably fall short of taking into account contextual features that link to embedded normative concepts.

A combination of the micro-linguistic analysis of discourse, ethnographic contextualization, and ideological concepts about larger processes is needed for a complete analysis of speech events and their contexts. Goodwin's study of courtroom negotiations (1994) illustrates that a pure linguistic (semantic) analysis of talk negotiations would miss indexical (pragmatic) features that point to social features of the event (such as the speaker's affiliation to a stigmatized social group, invoking frames including ethical arguments). The communicative event can in turn only be adequately understood by looking at its context before linking it to broader normative systems and perspectives. I propose that a methodological and theoretical triad of pragmatic-linguistic analysis, ethnographic contextualization and theorizing on the macro-level is needed for studying a speech community like the IGC and its communicative processes. The utterance alone cannot be adequately analyzed otherwise without looking at its broader context and taking into account 
ideological perspectives on the substantive issues under negotiation. All three aspects need to be combined in the analysis.

For the analysis of terminology, this has a number of implications. What Fabian describes for the study of emerging languages (Fabian 1977) equally holds true for taxonomic systems deriving from the communicative activities of a speech community primarily defined by those activities (and not, as the concept initially suggested, by descent, one shared language, or spatial limitation):

While classificatory relationships are undeniably involved in the determination of what a term 'means', investigations of emerging special languages whose formation can still be traced to concrete events, experiences and 'ideologues', compel us to ascribe equal importance to innovative, definitory articulations which become visible as the dogmatic closures of taxonomies. [...] Taxonomies [...] would have to be retrieved from texts, rather than from sentences and that may mean that not the dictionary of a language but its 'literature' is the appropriate source (making a dictionary one codification among others.) (Fabian 1977:194)

The shared activity of negotiating "traditional knowledge" among a varying number of actors, embedded in multiple institutional contexts, is in itself constitutive of a speech community that generates referential linguistic structures of shared terms and contested denotations. Looking at the elicitation of term-meaning relationships in documents or interview-responses in simple definitional modes $(\mathrm{X}$ means Y) would then be an equally insufficient source, much like Fabian's sentence-dictionary-complex. Instead, the opening of taxonomies and their contextualization in text-narrative relationships is a prerequisite for eliciting meaning as the dialectical relationship between terms and objects (Fabian 1977:183). Thus, the relationship between terms and their meaning is necessarily normative (as are taxonomies), shaping reality from subject-positions in a predominantly objectifying and naturalizing fashion.

This can best be illustrated by the discussions during IGC 14 and following meetings about the working method during subsequent sessions. The African Group Proposal ${ }^{180}$ called for "text-based negotiations" while Group B instead proposed using the term

[38] outcome-oriented deliberations (cf. [29])

It was introduced by the representative of the United States of America at IGC 14 during discussions on future work. ${ }^{181}$ This term has no "referent" in international

\footnotetext{
${ }^{180}$ WIPO/GRTKF/IC/14/8 Rev.

${ }^{181}$ Author's notes, IGC 14.
} 
negotiations or in IGC negotiations, leading the delegate of South Africa to remark that he was "puzzled by some of these concepts", that he "never heard of outcome-oriented deliberations" and that he "can't come back to my ministry with 'outcome-oriented deliberations' because of the seriousness of the issues".

The term has only weak indexical meaning and is a way to bypass a highly indexical referential frame and term - "text-based negotiations". At IGC 14, the term was used repeatedly by different actors (mainly the EU and the U.S.) and is documented in the report of IGC $14,{ }^{182}$ so it has a point of reference now in the official documentation.

Establishing such references can be viewed as an important strategy in international negotiations, as it contributes to the legitimation of terms and to the construction of stability and saturation by, for example, arguing the term has been used before and that this is a sufficient basis for shared understanding and use of the term. The phrase "outcome-oriented deliberations" is in this case used in conjunction with phrases such as "without prejudice to any outcome" and in contrast to "text-based negotiations", itself portrayed as too vague and ambiguous (cf. [27]). In a statement on this terminological opposition,

[39] [t]he Delegation of Brazil indicated that there existed a conflict between the terms "text-based negotiations" and "outcome-oriented deliberations". The term "text-based negotiations" could be considered as new language, since it had never been used before by this Committee. After nine years of deliberations, the Committee might need new tools and new language, such as "text-based negotiations", in order to move forward. The term "outcome-oriented deliberations", on the other hand, although it may be well-crafted or a new expression, could be perceived as non-committal language. ${ }^{183}$

While other delegations constantly tried to establish the conventionality of the phrase "text-based negotiations", the Brazilian delegate, belonging to a speeding up delegation, takes the step of portraying it as "new language" for the committee, while arguing that the "competing" term could be understood as "non-committal" language. Thus, he shifts the discussion on the two terms away from $\mathrm{RF}_{1}$ (technical discussions including procedure, specific definitions and other pragmatic aspects concerning the specific work the committee is to do) to $\mathrm{RF}_{2}$ (the indexically weak commitment to the IGC process). Dismissing the new term would, for that reason, not only denote a lack of terminological and procedural clarity, but also imply a rejection of commitment as the alternative term is "non-committal language".

\footnotetext{
$182 \mathrm{WIPO} / \mathrm{GRTKF} / \mathrm{IC} / 14 / 12$.

${ }^{183} \mathrm{WIPO} / \mathrm{GRTKF} / \mathrm{IC} / 14 / 12: 53$.
} 
In this example, specific terms are used as strategically deployable shifters mediating between the two main referential frames of the IGC. Taking into account this quality of terms central to IGC negotiations, their indexical and semanticoreferential meaning can only be elicited by looking at their narrative context, in this case the broad discussions of the two contrasting terms. The subject position of the speaker of an utterance influences this context and the resulting constellation in a major way. He plays a central aspect in portraying the relations between terms and the contextual objects they denote as already legitimated, thus lending these terms authority.

A number of important aspects can be distilled from this example: actors pursue strategies in international negotiations and use terms conducive to their interests. The use of these terms is contingent on the context of the utterance, both in synchronic and diachronic perspective. To be efficient, speakers have to take into account pragmatic and semantic meanings of terms, and have to consider relevant referential frames and audiences.

A number of important aspects can be distilled from this example: actors pursue strategies in international negotiations and use terms conducive to their interests. The use of these terms is contingent on the context of the utterance, both in synchronic and diachronic perspective. To be efficient, speakers have to take into account pragmatic and semantic meanings of terms, and have to consider relevant referential frames and audiences. They need to reflect the ambiguity, vagueness and instabilities of an emerging speech community and can use this latitude to their advantage by strategically deploying shifters, which calls forth a reaction from parties which are opposed. The creation of such a situation involves the intensification of instabilities demanding a response as well as the generation of stability: the speaker, by naturalizing his stance, can refer to a stable and authoritative position, while the recipient must make an effort to stabilize the situation.

Terms can only be understood by taking this context into account and by paying attention to the dialectical relationship between the specific term and its specific indexical meaning in "denotational text-time" (Silverstein 2004:634). In this example, they are used with the aim to make the decision between them a technical rather than an normative decision. Proponents of a given wording each try to portray them not as meaning-laden or intentional but as "natural" and as the only possible wording. Thus they shape reality from subject positions in a predominantly objectifying and naturalizing fashion. This can be achieved both by referring to abstract values (when all participants want the IGC to succeed, outcome-oriented deliberations are a good thing), or to discrete pragmatic functions (text-based negotiations are a common term and the natural next step in negotiations). Yet, the arguments can also be reversed, as the Brazilian example shows. And this requires delegations in opposition to change their strategy as well. 
The same dialectical relationship between terms and their meanings holds true for the analysis of pragmatic strategies. They can be viewed as the dialectical relationship between different desires and restrictions to these desires at a distinct moment that creates the possibility to realize a strategy, changing between stability and instability. Contextualization and the analysis of ideological perspectives here, perspectives on cultural property - are a presupposition for the analysis of utterances in the context, as they allow for an understanding of the speech community within which an utterance is realized. Pragmatic strategies are linked to terms and utterances, but they are also linked to social and political interests or desires that determine the shape of linguistic utterances. Limiting the scope of analysis to terms, their meanings and the pragmatic strategies they are part of misses the broader socio-political context that influences them.

Hence, the concept of the speech community is both useful and problematic. As Silverstein notes, pragmatic strategies "depend in the last analysis upon the shared understanding of norms for indexical elements in speech acts" (Silverstein 1976:48), meaning the normative implications of utterances need to be understood by at least some participants in the speech community. If nobody understands the pragmatic meaning of an utterance apart from the speaker, its indexical value is zero.

If we subscribe to an understanding of the speech community as the process of communicative interaction that reproduces and shapes rules of linguistic conduct, a "shared understanding of norms" depends on specific factors that influence the specific communicative exchange. There are already established rules and norms of communicative practice. There are also rules of interaction and interpretation that are in flux, contested, or vague. At any time during communicative events within the IGC, participants come from different linguistic and speech communities and thus do not necessarily share an understanding of all the indexical meanings of utterances during a communicative event. In such extremely heterogeneous and dynamic speech communities like WIPO's IGC, ${ }^{184}$ a "shared understanding of norms" is too limited a focus for analysis. What has to be taken into account as well are the strategic interests and ideological presuppositions of participants. The IGC as speech community would thus be constituted both by communicative interactions and the broader context.

A second aspect is the diversity of participants. The skill sets, level of competence, ideological presuppositions, motivations and "thought patterns" 185 that characterize each actor is very heterogeneous, as is how each actor perceives language, linguistic registers and linguistic choice. For an analysis of pragmatic strate-

\footnotetext{
${ }^{184}$ Some of them are professional diplomats, others experts in copyright, trademark, or international law, scholars from a broad range of disciplines, and NGO representatives with various professional backgrounds.

${ }^{185}$ See Mertz 2007 for an account of how one learns how to think like a lawyer in law school.
} 
gies or pragmatic qualities of utterances, this needs to be taken into consideration, as this is not an ideal-typical situation of a speech community in which a high degree of shared understanding can be presupposed. Neither Bakhtin's notion of "typical expressions" nor Fabian's dialectical analysis of taxonomies pay attention to the fact that in any given communicative event with more than two participants, there is more than one dialectical negotiation of taxonomies or attribution of meaning to typical expressions. ${ }^{186}$

Utterances in such communicative events of a speech community in a dynamic state have different simultaneous functions (Silverstein 1976:47). In any given communicative event, actors perform more than one function of an utterance simultaneously, consciously or unconsciously, intentionally or unintentionally. These functions are directed at a number of different audiences at the same time, meaning the indexical or referential meaning of an utterance is different from audience to audience. What can be distinguished are different dimensions of audiences involved in the IGC process as well as different functions relating to them. They can be used to signal positions and strategies, to build and maintain alliances between different delegations, and to establish hierarchies and pressure.

Taking the statement by Brazil as an example ([38]), what is signaled to the audience is not only the delegation's position with regard to the discussions of "text-based negotiations" and "outcome-oriented deliberations", but also the delegation's perspective on the IGC process as a whole, the pragmatic strategy of wanting to speed up negotiations, and the construction of an opposition. All these different functions influence communicative patterns and strategies significantly.

Interactional practices of the IGC are thus contingent on the multifunctionality of utterances, and responses to utterances must compensate for their multiple relevant functions. This requires creativity and going beyond structured interactional patterns. During the heated discussions on future work at IGC 14, representatives of stalling delegations such as Germany do so using personalized forms of address. A harsh critique of opposing delegations was countered by a courteous statement that touched on substantive questions and made use of $\mathrm{RF}_{2}$, but also used the first name of the representative of the African Group to relax a tense situation and signal a shared interest. This created the possibility of making a response that was essentially not in the interest of the opposed party, but was hard to respond to critically due to the use of a social index signaling a friendly exchange. Such unconventional forms of address can of course also be used by speeding up delegations.

In conclusion, the creative use of interactional practices draws its force from instabilities of the speech community. As outlined in Chapter 3, the maintenance of stability is of interest both to speeding and stalling delegations. Stability can be

\footnotetext{
186 Similarly, one might argue that even in communicative events with only two participants - including the reading of a book - there is more than one negotiation of meaning (Bakhtin 1983).
} 
maintained mainly by progressively moving forward, as the discussion of referential frames has shown. This also holds true for the practice of micro-editing that will be described in the following.

\section{Micro-Editing}

Micro-editing is part of more general tactics in international organizations. In contrast to the pragmatic strategies described above, the process of micro-editing does not concern whole utterances as instantiations of communicative patterns and strategies. Rather, it is the modification of small utterance fragments to change the indexical meaning of utterances in context.

As an example, during IGC 12 in early 2008, the decision process on future work of the committee did not take place in the main session but was brokered in meetings of regional groups. Their spokespersons communicated with one another via the chairman and the WIPO secretariat. At the end of this process, a written proposal was drafted which then had to be decided upon in the main plenary. Given the high uncertainty as to the conventions of this bartering process as well as the high stakes, actors in this setting relied upon micro-editing of text to channel their interests into these documents. This process is both in evidence in such small, closed group sessions preparing the decision making documents for the general session, but can also be observed during drafting exercises in the main plenary.

In the Group B regional group consultations, preparing the future work list at IGC 12, a great deal of strategic discussion took place, albeit in a highly relaxed, even familiar conversational tone. There was speculation on the specific intent of the session's chair in asking for a decision on this task, as well as on the possible moves to be expected from delegations eager to see quicker results.

The draft document on future work prepared by the IGC secretariat was carefully scrutinized. Upon encountering the formulation "in-depth work", one participant argued for crossing this out for fear that another two- week committee session, the format of some IGC meetings prior to IGC 12, might be the result; his fears made the room erupt in laughter. In another group meeting it was proposed to label a proposal by Group B with a new document number (i.e. WIPO/ GRTKF/IC/12/...) to signal progress. Similarly, a delegate proposed to replace the term "gap" in a document about the gap analyses on TK and TCEs with "interface issues", as this was a more ambiguous term that could strengthen the negotiating position of Group B. ${ }^{187}$

${ }^{187}$ Author's notes, IGC 12. 
The informality of conduct and the idioms used in this setting, such as "kicking the can further down the road" or "breaking some china", illustrates a high level of awareness of the emergent habitus of the committee and an offhand awareness of the power of effectively used colloquial expressions. It also allowed delegations to openly discuss strategies for main plenary discussions. In one regional group meeting, it was argued that a proposal by the African Group on future work should not be discussed substantively. Instead, it was proposed to argue that Group B needed "more time to evaluate" the proposal, as one should not "ask for information because we might get it." Needless to say, the group observed here consisted of delegations that felt they had the upper hand in this setting. Microedits can have a significant influence on IGC meetings, be it by effectively establishing referential frames, deploying shifters or by framing decisions in a way conducive to one's interests.

Three examples illustrate the use and effects of micro-edits, all of which stem from the initial draft document on future work prepared by the IGC secretariat and the reactions to this document by Group B delegations at IGC 12. In this small setting in Room B, delegates huddled together in small groups, discussing specific phrases and formulations and trying to take all possible implications of the paragraphs into account. The Group B meeting was chaired by a representative of the U.S. permanent mission in Geneva. Aside from the informal character of the meeting, there seemed to be a general shared understanding of the regional group's goals and motivations. Some of the more active delegations (Canada, the U.S.) were assisted by younger staff members who provided copies of documents, prepared texts and modified proposals according to the discussions. The specific instances of micro-edits were thus prepared in these informal settings and later introduced into the main plenary.

The first example addressed the scope of documents and prior work to create the gap analyses for TK and TCEs. The initial draft prepared by the secretariat read as follows:

[40] The Intergovernmental Committee reviewed the progress made on its substantive agenda items at the current session, and agreed that: (i) concerning item 8 (traditional cultural expressions/expressions of folklore (TCEs/EoF)), the Secretariat will prepare, as the working document for the next session of the IGC, a document that will describe $(\ldots)$

In contrast, after discussions Group B successfully requested to change the wording to:

[41] The Intergovernmental Committee reviewed the progress made on its substantive agenda items at the current session, and agreed that: (i) 
concerning item 8 (traditional cultural expressions/expressions of folklore (TCEs/EoF)), the Secretariat will, taking into account the previous work of the IGC, prepare, as the working document for the next session of the IGC, a document that will describe (...)

By inserting a clause such as "taking into account the previous work of the IGC" into the working document, the scope of documents that must be considered is significantly expanded. This leads to an increased ambiguity of the resulting document, which is in the interest of stalling delegations.

Similarly, the following change of wording has the effect of stalling negotiations by way of broadening the scope of information the committee has to deal with:

[42] At its thirteenth and subsequent sessions, all three substantive items of the Committee's mandate (genetic resources, TK and TCEs/EoF) should be discussed in depth and that the time allotted to each item be balanced.

The final version of this paragraph read:

[43] At its thirteenth and subsequent sessions, including any intersessional sessions, all three substantive items of the Committee's mandate should be discussed in depth and that the time allotted to each item should be balanced.

By adding the phrase "including any intersessional sessions", stalling delegations can insure that inter-sessional work will need to be considered in equal measure with regard to the three substantive issues of the IGC. Even if some parties might see the possibility of achieving tangible consensus in one of the areas discussed, the insertion insists such progress cannot be made at the cost of another area, either during the bi-annual meetings or even during any formal intersessional work. This inclusion is especially noteworthy since the discussions on GR lag behind, and thus any progress in the other areas could be stalled by this issue.

A third example for micro-editing relates to the softness of verbs. With reference to the introduction of inter-sessional working groups as proposed by the African Group, the initial draft from the secretariat read:

[44] With a view to accelerating the work of the Committee in accordance with its mandate, at its thirteenth session the Committee will develop proposed modalities and terms of reference for the establishment of intersessional mechanisms or processes $(\ldots)$ 
The wording of this paragraph that is also part of the decisions of IGC $12^{188}$ was altered after Group B discussions and consultations between the regional group coordinators to read:

[45] With a view to accelerating the work of the Committee in accordance with its mandate, at its thirteenth session the Committee will consider taking a decision on proposed modalities and terms of reference for the establishment of intersessional mechanisms or processes $(. .$.

The change from "will develop" to "will consider taking a decision on" nicely reflects the efforts of Group B to stall the negotiations. By using non-committal and ambiguous language, the process is to be prolonged and specific decisions, as well as the drafting of documents needed for progress, are delayed. The reluctance of Group B countries to take a binding decision on the given subject is reflected in this change of wording.

The opposing parties are well aware of the significance of these micro-edits. This creates a dynamic of conceding and retaining. You have to concede some of your opponents "linguistic moves" in order to retain some of yours. It is thus not about winning (as winning would imply that there is no consent), but about obtaining a strategically advantageous position for the next round of negotiations. Although the intentions are often clearly recognizable, pragmatic strategies and micro-edits allow for a continuation of the IGC process at a slow pace and with dim prospects for coming to an agreement. They are a way for stalling delegations to keep issues of TK and TCEs largely limited to WIPO while not making too many concessions.

The presupposition for such thinly veiled communicative strategies is their vagueness and ambiguity. In this respect, micro-edits are similar to shifters, in that both not only differentiate between positions, but also map commonalities and the common ground between actors who otherwise hold incommensurable positions. They mediate between different actors, contribute to the cohesion of the committee and allow for the expression of fundamentally different viewpoints. Thus, they are a way for actors to exchange views that can differ from each other extremely, while at the same time creating a discursive space that allows this exchange. The specificities of diplomatic negotiations allow for pragmatic strategies balancing between distinct interests and motivations. While the intent behind micro-edits like changing "will develop" to "will consider taking a decision" is well-understood by participants in the IGC, its specific instantiation prevents delegations from being held fully accountable for this implicit stalling of the IGC process. The veiled intention behind such indirect speech acts must be inferred from its context. This inference can be understood as a "buffer" making it possible for stalling delega-

188 WIPO/GRTKF/IC/12/9. 
tions to realize their strategies in order to slow down negotiations. On the other hand, this buffer prevents speeding delegations to openly critique them for doing so, as the changes in wording are ambiguous and can be attributed to other external factors, such as the need for further consultations and discussions to achieve more clarity.

The practice of micro-editing is not limited to regional group meetings. In IGC sessions beginning at IGC 14 in June/July 2009, the discussion of specific paragraphs was shifted to the main plenary session. Projected onto a screen behind the dais, delegation after delegation commented on the African Group proposal for a new IGC mandate for the 2010/2011 biennium. ${ }^{189}$ Although this mode of drafting texts is common to international negotiations, the session's chair as well as the WIPO secretariat initially struggled with implementing this approach. The chair was not wholly satisfied with this mode of work from the beginning and stated a number of times that "this is not a drafting exercise", although delegations subsequently engaged in just that. He repeatedly complained that he could not see what was projected onto the screen behind him. Numerous offers by delegations to provide him with a laptop or a second screen so that he could monitor what happened behind his back were ignored.

The specific methods for documenting the statements and comments onscreen also had to be figured out. It was unclear, for instance, whether new text should be bracketed or underlined, or whether the member state requesting the change should be noted in a footnote or in the continuous text. These uncertainties have been addressed, so that starting with IGC 17, deletions are marked by brackets, additions by underlining, both preceded by the delegation's name set in upper case:

[46] Peoples and Communities, INDIA, MBOSCUDA, MEXICO, TRINIDAD AND TOBAGO [for example] including Indigenous Peoples, Local Communities, Cultural Communities, and/or MBOSCUDA First Nations IRAN individual groups and families in whom INDIA [the custody], and safeguarding of the traditional cultural expressions/expressions of folklore are INDIA [entrusted or held] presumed to be vested in accordance with $[\ldots]^{190}$

As micro-edits, the amendments and requested deletions can be understood as instantiations of communicative patterns and strategies related to interests and power. For instance, the changes requested by India to this passage on the definition of beneficiaries in WIPO/GRTKF/IC/16/5 ("The Protection of Traditional Knowledge: Revised Objectives and Principles") hint at domestic policy issues and

\footnotetext{
${ }^{189} \mathrm{WIPO} / \mathrm{GRTKF} / \mathrm{IC} / 14 / 8$ Rev.

190 Author's notes, IGC 17.
} 
the relation between indigenous groups (or adivasi) and the state in India. ${ }^{191}$ Similarly, a proposed amendment by Iran can be read as the awareness of potential cultural resources tied not to communities but to individuals in the Iranian context. Micro-edits thus go beyond the level of strategy within IGC negotiations and relate to the political and economic interests of member states.

For example, at IGC 17, the delegation of Trinidad and Tobago requested to add

\section{[47] works of mas}

to the list of forms constituting the subject matter of protection for a possible legal instrument. The representative argued that this specific form of TCEs was not adequately reflected in the document, though other delegations referred to the phrase "tangible and intangible" as encompassing this form as well. The example indicates that member states want to see their interests accurately reflected in WIPO documents, but it also leads to highly specific formulations, undermining the intent of creating broad, encompassing legal tools. This makes it difficult to draft and decide on texts, as the legal implications of all aspects in these texts need to be considered by member states.

Speeding up delegations can thus also stall negotiations by being too specific in their micro-edits. The distinction between stalling and speeding delegations is only an abstraction of a more complex constellation of actors and motivations, for at times the strategies and arguments employed by delegations interested in substantial results of the negotiations are ineffective or even cause a deceleration of the process. This relates to the complex interplay between the interests of member states, ILCs, NGOs, regional groups and the two basic strategies (speeding and stalling). If a given delegation or organization is part of speeding up delegations but views the position of other speeding delegations as problematic, the specific interest of the delegation can have more weight than the general interest in coming to an agreement. The proposed inclusion of "works of mas" is one example for this: while it serves the national interest of Trinidad and Tobago and other states, ${ }^{192}$ it takes some speed and momentum out of negotiations as it causes discussions about its reasonability.

Another example concerns the relation between ILCs and the African Group: as the main proponent of a legally-binding agreement for the protection of TK and TCEs, the African Group often portrays itself as representing the bearers or holders of cultural property as well. However, many IGCs contest this notion as it is counterproductive to their interest of maintaining and controlling their TK and TCEs autonomously. They thus contradict some proposals by the African Group,

\footnotetext{
${ }^{191}$ See Fenelon and Hall 2008, which includes a number of case studies.

192 Barbados and Jamaica supported the amendment as well.
} 
although this leads to a weaker position of speeding up delegations. Micro-edits thus mediate between different levels and aspects of negotiations, and as instantiations of communicative strategies they balance various interests and motivations of participants as well. For that reason, there is a hierarchy of strategies that at times subordinates the general strategies of speeding and stalling.

To come back to the specific process of micro-editing as a way to either speed or stall negotiations, a number of examples at IGC 14 illustrate how delegations make use of utterance fragments in order to edit their interests into documents. The discussions centered around the African Group proposal ${ }^{193}$ show that the modification of text, even in small dimensions, has relevant implications for the course of negotiations. Similar to opening statements, it also permits an insight into the perspectives and strategies of actors in IGC negotiations. The second sentence of the original text of the African Group proposal - clearly aiming at fast progress and establishing a mode of work preparing a legally binding international instrument for the protection of GRTKF - read as follows:

[48] The Committee will undertake during the next budgetary biennium (2010/2011) text-based negotiations on genetic resources, traditional knowledge and traditional cultural expressions.

During the discussions of this passage at IGC 14, a number of delegations made comments with regard to the specific wording. While the text was projected onto the screen behind the dais, representatives requested amendments, changes and deletions to this sentence. The Australian delegation proposed to rephrase the text as follows:

[49] The Committee will undertake during the next budgetary biennium (2010/2011) text-based negotiations without prejudice to the outcome, including a possible legally binding instrument on genetic resources, traditional knowledge and traditional cultural expressions.

The addendum to the paragraph leaves the door open for stalling delegations to contradict arguments by speeding delegations that text-based negotiations are a clear indication for the drafting of a legally binding instrument. Other types of outcomes, especially a non-binding convention or a declaration, are included in the edited statement, thus reducing the stakes for stalling delegations not interested in substantial changes to the intellectual property system. Similarly, the EU requested the paragraph to be rephrased as follows:

${ }^{193}$ WIPO/GRTKF/IC/14/8 Rev. 
[50] The Committee will continue its work and undertake during the next budgetary biennium (2010/2011) text based negations outcomeoriented deliberations on genetic resources, traditional knowledge and traditional cultural expressions, based on their impartial treatment and noting the different levels of development in the texts.

As outlined above, outcome-oriented deliberations lack the implication of working towards a text as the basis for a potential legal instrument. The delegation from New Zealand, in a similar vein, proposed to replace text-based negotiations with "the development of text." On the other hand, the representative of Brazil argued that the passage should be complemented by a formulation differentiating between the three substantive issues of the IGC:

[51] The Committee will undertake during the next budgetary biennium (2010/2011) text-based negotiations on genetic resources, traditional knowledge and traditional cultural expressions, taking into account the different levels of development reached by the three substantive topics and the different levels of development of the texts indicated in the third paragraph.

The rationale behind this proposal was that by factually separating TK and TCEs from GR, it was possible to speed up negotiations on the first two issues where substantial progress was more likely. Adding this formulation as a qualifier into the text, speeding delegations would thus be able to refer to it and argue for a quick agreement on TK and TCEs independent from the work on GR that was lagging behind.

In the process of constructing text by micro-edits, proposals are edited in order to reflect strategic perspectives on the consequences implied in the text. "Textbased negotiations" are rephrased as "outcome-oriented deliberations" or "the development of text" or fully discarded, as they are regarded as conceding too much to the parties in opposition. These "drafting exercises" are quite common in the UN system, and call for the competence, if not sophistication, to phrase one's desired perspectives at the appropriate linguistic micro-level. The implications of terminology need to be considered closely, such as the - at least in the UN system - shared understanding that "text-based negotiations" refers to the drafting of a convention or a treaty. "The development of text", or for that matter "outcomeoriented deliberations" have different implications - first and foremost not the drafting of a convention or treaty. While on the first sight these distinctions seem to be rather simplistic, the extensive amount of work that was put into these drafting exercises illustrate that even minor changes of linguistic fragments can have major implications on the further proceedings - a fact that is acknowledged by the actors involved and taken into account when drafting texts. Interviews and conversations with IGC participants indicate they are quite cognizant of and very reflective about these practices. 
Like opening statements, micro-edits can be understood as boundary objects mediating between stability and instability in the process of IGC negotiations. They maintain a common ground and a shared understanding that allows actors to stay at the negotiating table and continue their deliberations despite radical differences in perspective. This is possible through the interplay of referential frames in the sense of conceding and retaining - and the communicative frame. They permit the expression of interests and strategies in a manner that prevents overt reproaches or disputes. By editing small utterance fragments, actors can frame texts and passages of text according to their perceptions and interests. As instantiations of communicative strategies, micro-edits are compensatory mechanisms, as they permit conflicting utterances while maintaining the IGC as a speech community. Furthermore, they are highly variable strategically deployable shifters, The meaning of micro-edits is mostly at the pragmatic and not the semantico-referential level. Phrases like "without prejudice to the outcome, including a possible legally binding instrument" ([49]) gain their power not from semantic meaning, but because they point a more general strategies within the IGC while maintaining coherence among the participants.

To understand these edits, it is necessary to analyze not only the utterances and the IGC as its context. What has to be taken into account as well is the broader ethnographic context, issues of domestic policy and diplomacy, including ideological presuppositions about the substantial issues negotiated. The following chapter will shed light on this interplay using the example of different perspectives on traditional knowledge in IGC negotiations. 


\section{Multiperspectivity \& Differentiation}

As shown in Chapter 4, the relation between terms and their contexts is of great importance. This chapter surveys perspectives on traditional knowledge of indigenous communities, and highlights the connections and interdependencies between these perspectives. This chapter also outlines the different interpretations of terms used in WIPO's IGC, showing specific social conceptions implicit in these interpretations, thereby shedding light on the constitution of IGC terminological constellations.

These specific social conceptions can influence negotiations and the interpretation of the IGC's subject matter at various levels. ${ }^{194}$ Opening statements, referential frames and micro-edits all draw from such conceptions, and the instantiations of communicative patterns and strategies can only be adequately understood by taking into account participants' ideological presuppositions about the substantive issues discussed in the IGC. Although these issues - TK, TCEs and GRs - are relatively marginal in face of the mainly procedural discussions in IGC sessions, participants's perspectives on these issues contribute significantly to the negotiation process. What will be examined here are the five different perspectives of differentiation and confirmation that actors in the IGC take with respect to TK and TCEs.

Two prefatory remark are in order. First, because there is a close connection between the concepts of TK or TCEs and the attribute "indigenous" in IGC negotiations (sometimes called "indigenous knowledge"), it is necessary to combine these concepts when analyzing them. "Bearers" (Carneiro da Cunha 2009: 9) of TK in the context of the IGC are in most cases indigenous communities. An

${ }^{194}$ For a discussion of tradition and value in the context of the IGC, see Groth 2009. 
analysis of perspectives on traditional knowledge therefore inevitably has to deal with questions of indigeneity. However, this shall not attempt to fully grasp the complex usages and meanings of indigeneity. Rather, the specific terminology employed in WIPO IGC negotiations will be used to show how the interrelation of traditional knowledge and the attribute "indigenous" influences actors' perspectives.

Second, examining multiperspectivity in diplomatic - and therefore strategic contexts raises questions about the intentionality and agency of linguistic utterances and negotiation strategies: what do actors try to achieve by framing subject matter in a certain way? This question points back to the relationship between direct and indirect speech discussed at the beginning of Chapter 4, a relationship centered around intentionality and metapragmatic awareness. Of interest to this study are mainly the four constellations in which: (1) the intentionality of an utterance is very obvious, so that speaker and audience are (mostly) aware of the implications and its indexical meaning. There is also a metapragmatic awareness since the pragmatic features of the utterance are commonly understood; (2) the speaker of an utterance is aware of its pragmatic features and potential implications, but the audience may not be completely aware of the pragmatic meaning of the utterance, and only anticipates the intended implications; (3) the speaker of an utterance is only partly or unconsciously aware of the indexical meaning of an utterance, and the utterance is part of a set of habitualized phrases or linguistic strategies. The audience, depending on its level of communicative competence in the specific kind of communicative event, may be aware of this strategy and perceive it as common for the context of negotiations; (4) the speaker of an utterance is unaware of its pragmatic features, but there is a reproducible effect that can be observed in correlation with such utterances. If the speaker realizes the pragmatic function of this type of utterance, his awareness of it can rise so that the utterance can be strategically and intuitively deployed. These four constellations are typical for communicative events, including communicative patterns with identifiable intended or perceived pragmatic qualities. Of interest for this study is an audiencefocused view of communicative events and associated pragmatic strategies that takes into account the dialectics between the speaker of an utterance and his audience.

Multiple perspectives on TK and TCEs find their expression in IGC communicative events. Whether explicitly or implicitly, the instantiations of communicative strategies and patterns (for instance, specific stalling tactics, or the framing of TK as primarily a matter of economic and cultural values disconnected from social and political struggles and inequalities) reflect these perspectives. In analyzing them, one must determine whether their indexical meaning of an instantiation or perspective is reflected by its speaker, and thereby whether the observed language use is conscious and intentional, or unintentional, habitualized and incidental. This is especially difficult because the IGC consists of multiple audiences with varying 
levels of communicative and professional competence in the implications of strategic language use. The problem is further compounded by the IGC's emergent nature as a speech community: while some communicative conventions are prestructured as part of UN norms, others are flexible and constantly changing. Analyzing substantive discussions and perceptions along with abstract strategic language use poses the additional problem that actors not only depend on their communicative and professional skills (that is, to speak like a diplomat and weigh the implications of speech like a lawyer), but also on a sensitivity to different conceptions of TK and TCEs.

I argue that one can use the notion "mutual partial intelligibility" - used in linguistics when speakers of different languages understand each other to some degree without being capable of speaking each others' language - to understand this situation. In IGC negotiations, the arguments and basic ideological and substantive stances of opposed delegations and participants are understood, yet the fundamental presuppositions which lead to the different perceptions are lacking. However, the negotiations do reflect instantiations of diverging perspectives in communicative events. These instantiations are characterized by their differentiating relationship to each other: a given perception of TK or TCEs is contextually constituted by its presumed counterpart in negotiations. It is therefore possible to map the recipient design of communicative strategies onto the level of the three main IGC issues such that these different perspectives constitute each other in a dialectical relationship.

Susan Gal and Judith Irvine describe these processes of differentiation as "fractal recursions" involving "the projection of an opposition, salient at one level of relationship onto some other level" (Irvine and Gal 2000: 38). Taking the example of the public/private dichotomy, Gal clarifies:

To be fractal, a distinction must be co-constitutive, so that the terms - like right and left or east and west - define each other. Such coconstitutive contrasts can be used to organize virtually any kind of social fact: spaces, institutions, bodies, groups, activities, interactions, and relations. Furthermore, whatever the local, historically specific cultural prototypes or images that motivate oppositions like public and private, the distinction can be reproduced repeatedly by projecting it onto narrower and broader comparisons. This always involves a change in perspective by those making the comparison. Fractal recursions are repetitions of the same contrast but at different scales. (Gal 2005: 26-27)

The dialectics of strategic communicative behavior in international negotiations operate in a similar way with regard to ideological presuppositions about the subject matter. Switching referential frames or using strategically deployable shift- 
ers in micro-editing processes always implicitly involves a second scale separate from the specific negotiations. These processes also involve substantive stances and perspectives on what is being negotiated: the procedural scale is superordinated to a substantial scale that is made explicit only at times. The constitution of such scales occurs at, and draws from, different levels, so stalling and speeding up tactics in negotiations depend on each other. Since the scale of substantive perspectives determines the procedural scale, examining only the procedural level, meaning the instantiations of communicative strategies and patterns in negotiations, overlooks the essential scale. Thus, the EU's position cannot be understood only in terms of the strategic value or pragmatic meaning of utterances in the context of negotiations; value-related and structural factors must be considered as well. Among these factors are the valuation of individual creativity in Western societies, and the fact that the current intellectual property system benefits the economic interests of industrialized nations. ${ }^{195}$

The complementary nature of oppositions constituted as fractal recursions coincides with another situation in which overt distinctions potentially counterproductive to the interests of speakers are veiled. Irvine and Gal describe these situations as different forms of erasure that blur dichotomizing distinctions on the various scales:

The process of fractal recursion allows and indeed invites erasures. In general, erasures are forms of forgetting, denying, ignoring, or forcibly eliminating those distinctions or social facts that fail to fit the picture of the world presented by an ideology. [...] One level of distinction can be foregrounded at the expense of another, eliding or ignoring that there have been several nested contrasts made. [...] In these cases, fractal recursions focus on similarities between contrasts made at different scales, and the differences are ignored. The several (somewhat different) distinctions involved in the reiterations can then be conflated into a single contrast. But erasure can also operate in a way that does not entirely obliterate newly created nestings in existing distinctions but merely highlights their similarities. This makes new contrasts seem like old friends returning in somewhat different clothes, novel versions of familiar phenomena. (Gal 2005: 27)

In IGC negotiations, one form of erasure is the switching of referential frames: by stressing stability on the indexically weak level, differences at another level are portrayed as being constituted not of substantive discrepancies, but rather of insubstantial technical and procedural discrepancies that can be solved over time. Fractal

\footnotetext{
195 As bilateral agreements and developments on the national level show, industrialized nations are not per se against agreements over TK, TCEs, or GR. However, they are cautious as to the implications an international and legally-binding agreement might have for the intellectual property system.
} 
recursions and erasure are especially important for the analysis of IGC negotiations because of how they relate to intentionality. Because they expand the scope of analysis to different scales, these two processes exacerbate the already problematic distinction between intentional communicative behavior and utterances that have indexical meaning but lack metapragmatic awareness. The relationship between different scales emphasizes the need for both a contextualized study of negotiations (encompassing linguistic analysis as well as an ethnographic account), and for macro-level reflection on the broader ideological presuppositions that help construct observable communicative behavior.

The relationship between scales also makes analyzing the intentionality of communicative behavior more complex, especially given the emergent nature of the IGC as a speech community. With regard to intentionality, Duranti argues that

[f]or anthropologists, the crucial issue is whether it is possible to separate intentional acts from the cultural context in which they are produced. Since the route to interpretation relies on conventionality as much as on intentionality, it is possible, and in fact quite common, that an audience may respond to what they judge to be contextually relevant conventions, ignoring the issue of the speaker's intentions. Ethnographers have also shown that the view that one can know what goes on in another person's mind is not shared by all cultures, and a difference in power or authority, sometimes defined in terms of expertise, may grant some individuals or groups the right to interpret while depriving others of the right to reclaim their original intentions. (Duranti 1999: 135)

This view is essential for the study of IGC negotiations and for the discussion of intentional or unintentional communicative acts. In the context of the IGC, Duranti's idea of conventionality is heavily contested. At the heart of any definition of conventional communicative behavior lie questions of power, since the prerogative of interpretation can be a crucial advantage in pragmatic strategies and negotiations. Any such definition is thus unstable.

The concept of intentionality is equally contested and vague, as intentionality (consider the notion of erasure) is sometimes blurred or veiled, contingent on various scales of interaction and not necessarily shared within the speech community. In strategic communicative behavior, however, the process of ascription and self-ascription is often intentional (Barth 1969). Though fieldwork indicates that actors in the IGC are sometimes fully cognizant of the strategies they employ, I would argue that the dialogical constitution of communicative events (in the sense of both fractal recursions and habitualized communicative practices) is equally important in this context. Furthermore, ex post facto rationalizations of successful strategies cannot be excluded. This is because it is more important to observe the 
communicative strategies actually employed in the IGC than to hypothesize about whether an utterance was made with an intentional strategy in mind. The fluid character of the IGC as a speech community leads to a constant shifting of communicative conventions, so any pragmatic strategies observed are more an expression of reactions to this fluidity and contingency on the basis of stable intentions than the reflexive use of pre-structured behavior. This contingent and dynamic relationship between actors and their strategies is illustrated by Elizabeth Povinellis' work (2002) on recognition practices in the context of Australian indigeneity. She shows that overtly direct claims to cultural property can be counterproductive to the interests of indigenous groups, as such claims are viewed as not "authentic" to these groups. ${ }^{196}$ These limitations on discourse prevent overt strategies of positioning using the notion of indigeneity.

Taking into consideration this complex relationship between terminology and utterances and normative presuppositions about substantial issues, the following will outline a survey of different perspectives on the traditional knowledge of indigenous groups in IGC negotiations.

\section{Stigma}

In many African countries, the term "indigenous" refers to those who originally lived on the land - it is a temporal difference to something else, a dichotomy that is only functional with a counterpart in the past, in this specific case with colonialism. As a discursive marker, the attribute "indigenous" is open to interpretation and exploitation. It is used in varying contexts for different purposes, of which the dichotomy between colonialism in the past and sociopolitical regimes of the present is only one example, albeit a very powerful one. Indigeneity is a relational concept:

[S]ocial groups become indigenous or aboriginal or native by virtue of the recognition that someone else arrived in a place and found them or their ancestors 'already' there. (Pratt 2007: 398)

Indigeneity exists and is constituted by virtue of difference, and the representation of difference exists and is constituted by opposition. As a semantic label, the poles of this dichotomy point to interpretations and ideologies that attach positive or negative meaning to it.

During the first IGC in 2001, the Zambian delegation emphasized this dichotomy and argued for an expansion of the term:

196 Povinelli 2002; see also Trouillot 2003. 
[48] In the Zambian context, Indigenous and Local Communities refers to people who by necessity or own desire depend on living off their ancestral land and/or live under traditional authorities and share common cultural values. These must include communities in urban and peri-urban areas of Africa. Some members of urban and periurban communities have strong ties with people who live off their ancestral land and/or live under traditional authorities and share common cultural values. Traditional knowledge systems, traditional knowledge and innovations are manifested through traditional practices and lifestyles. The introduction of foreign values, foreign religions, changing lifestyles and the legacy of colonialism have contributed greatly to lowering the status of traditional knowledge systems, traditional knowledge and innovations in Zambia. ${ }^{197}$

The delegation argues that the viable difference exists between foreign or external influences in contrast to TK systems tied to the past and including geographic materializations of the past. Similarly, a WIPO publication on "Traditional Knowledge \& Indigenous Peoples" stresses this dichotomy of perspectives on TK as the difference between colonial actors and indigenous peoples:

[49] Within this context, perceptions of indigenous knowledge rested on the dialectical tension between the "colonial" views of indigenous historic, cultural, and intellectual knowledge and the understanding of these bodies of knowledge as expressed by indigenous peoples themselves. (Popova-Gosart 2009: 17)

This dichotomizing view is commonly held by stalling delegations in IGC negotiations. As outlined above, it coincides with the construction of different actor categories in communicative events where ILCs and their knowledge are portrayed to be spoken for by speeding delegations in contrast to external actors either causing or not acting against acts of misappropriation and misuse. A typical instantiation of this perspective can be found in a statement by the delegation of Senegal on behalf of the African Group at IGC 14:

[50] The Delegation reaffirmed that only the adoption of a legally binding international instrument could guarantee the effective protection of folklore and traditional knowledge as well as genetic resources of indigenous and local communities of Member States. ${ }^{198}$

\footnotetext{
${ }^{197} \mathrm{WIPO} / \mathrm{GRTKF} / \mathrm{IC} / 1 / 12: 1-2$.

${ }^{198}$ WIPO/GRTKF/IC/14/12:5, my italics.
} 
The aspect of claimed representation is of importance for this dichotomy, as it conceptualizes the debates of the IGC as a dichotomous conflict between speeding delegations and ILCs on the one hand and other actors on the other hand.

On one level the dichotomy between indigenous and non-indigenous is national, domestic policy as related to history and to the confined territorial space demarcated on maps - however artificial those demarcations are culturally. As such, the colonial (or outsider) versus indigenous dichotomy influences social relations and the institutionalized forms of mutual recognition (Hegel 2006). The dichotomy also fleshes out power relations between urban elites, the holders of traditional knowledge, and of everybody in between and beyond. The idea of representation, meaning the state as the representative of ILCs and their cultural resources, reflects these power relations on the national level.

At IGC 16, the representative of the ILC Indian Council of South America (CISA) argued that at the national level, the indigenous/non-indigenous dichotomy is both highly problematic and powerful:

[51] What needed to be avoided were so-called national authorities where the colonizer was effectively making the law and reducing the ability of the indigenous peoples to be protected. He stressed that the indigenous peoples were recognized as colonized peoples, and that national authorities were actually colonial authorities. ${ }^{199}$

Thus, as a political concept, it points back to the past to have an influence on the present, to be used as political leverage. A large number of non-governmental organizations that deal with these power relations and the inequalities they cause are a vivid demonstration of how powerful this concept is.

In its negative connotation "indigenous" is an attribute that is imposed on a social group as a stigma: you are indigenous, therefore you are backwards and not modern. Its semantic proximity to and frequent conjunction with the notion of "community" as an outdated form of social structure points to the way in which it is conceptualized as negative ideological residue of pre-modernity:

As a rule, groups represented as "communities" are comparatively isolated, subaltern, and not considered to be viable autonomous collective subjects. Indeed, "community" is in part a euphemism conferring dignity and value on groups in a negative position: it is a verbal gift from the rich to the poor. At the same time, insofar as the label implies a refusal of individualism, it distances its referent from modernity. (Noyes 2006: 29)

${ }^{199}$ WIPO/GRTKF/IC/16/8:45-46. 
In this terminological constellation, indigenous communities are construed as distancing themselves from the promises of modernity and adhering to the past without embracing and contributing to the present. While the concepts of traditionalism and conservatism are commonly linked to the idea that something from the past is pursued to redound to the benefit of society, the notion of community as a social actor grounded in the past is frequently associated with isolation, subalternity, and reproduction rather than with innovation.

Numerous actors at the IGC, especially those representing ILCs and NGOs, make reference to indigeneity as a stigma:

[52] In the post-colonial context, indigenousness remained a characteristic that denoted an inferior and temporary social state of peoples, now approached as territories possessed by their respective states. This characteristic did not reflect the actual conditions of the indigenous societies - conditions that differed significantly across the world and, in many instances, even within one country. The later proposed theory that all post-colonial peoples were equally victims of the colonial regimes and should be uniformly deemed indigenous only enhanced the abstracted character of the term indigenousness. (Popova-Gosart 2009: 23)

[53] The colonial project largely succeeded in depicting TK as barbaric, heathen, and satanic. Although the formal structures of colonialism have been dismantled, resurgent religious fundamentalism and the hangover of colonial mindsets make it very difficult for TK and forms of CE [cultural expressions, SG] to take their pride of place. ${ }^{200}$

Both passages stress the construction of ILCs and their knowledge as inferior to other forms of knowledge and social organization on the one hand and the persistence of this constellation on the other hand. The derogatory view on ILCs and TK is especially present in the realm of cultural creativity. As Valdimar Hafstein observes, not only European peasants, but also colonial subjects were (and are) denied the ability to artistically create: "The subaltern do not produce, they reproduce." (2004:79).

In consequence, indigenous communities are in a Catch-22, with their indigeneity accusation and impediment at the same time. As the Other in a differentiation not of their own making, they are confronted with a dissociation that is constructed as reprimand: you are indigenous, therefore you are not modern - and, therefore: we are modern. ${ }^{201}$ Employing this notion of exclusion makes it possible to distance

\footnotetext{
200 WIPO/GRTKF/IC/10/INF/5(d).

201 See Bauman and Briggs 2003 for a discussion of the connection between language ideologies, modernity and tradition, and the perception of others.
} 
oneself by ascribing a negative social position to the Other, here the indigenous, and placing it in the past (Fabian 1983). Concurrently it places oneself at the other pole of this dichotomy in the present and as a part of modernity.

In terms of cultural creativity, with the rise of individualism comes an acknowledgement of individual innovation. Such innovation is denied to the Other:

[...] creativity and originality were the privilege of the bourgeoisie, while the masses were unoriginal and could only transmit the songs and tales of earlier generations. The art of the common people consisted only of copies. (Hafstein 2004: 79)

What Hafstein describes for the European concept of the original author in contrast to the unoriginal communal subject is analogously true for the relationship between indigenous communities and their self-proclaimed modern counterparts, at least in this specific constellation. This is illustrated by a passage from a work regarded as one of the foundational documents of the WIPO IGC process.

In one particular instance, a lawsuit was filed in Australia in 1989 by an indigenous artist claiming a T-shirt manufacturer had used his artworks without permission. Prior to this case, it was generally assumed that indigenous artworks were not protected by copyright:

[54] Indigenous artworks were not "original" because they are based on traditional creation designs; they are passed on through the generations and are not the independent creative effort of the individual artist. (Janke 2003:52)

The case was decided in favor of the indigenous claimant, and it was argued there was a large amount of individual creativity in the respective artworks.

Yet, the underlying notion that traditional knowledge and folklore do not satisfy the requirements of originality persists in WIPO negotiations and in national settings. As Gregory Younging of the Creative Rights Alliance argued before the indigenous panel of IGC 17 in December 2010,

[55] [d]espite the advances made by knowledge systems throughout the Indigenous world, the Western world's general response throughout the colonial and most of the post-colonial periods was to dismiss the value of TK. Since only European people could progress, all Indigenous knowledge was viewed as static and historical. ${ }^{202}$

${ }^{202}$ WIPO/GRTKF/IC/17/INF/5(a):3. 
The influence of this perspective of differentiation and the allied negation of innovativeness has considerable influence on international processes that try to agree how to protect traditional knowledge and folklore.

Of course, the practices of differentiation that are at play in this constellation are not bound to the national level. All social encounters draw from broader narratives and discourses, so though their status as viable social entities is sometimes denied to indigenous communities, socialization with these communities nevertheless exists. To exist, the self-conscious subject needs to recognize other self-conscious subjects as such (Hegel 2006: 120-136), and intersubjectivity depends on mutual self-consciousness. The attribution of otherness, as delimiting as it might be, is always acknowledgement of entanglement with other self-conscious subjects, so in practice, there is more social practice involved than mere narrative. ${ }^{203}$

As property relations are social (as a process of recognition and differentiation of desires towards an object), so are the practices of differentiation. They are manifested in social events and rituals (in the sense outlined by Silverstein 2004) and as such influence communicative practices in and between social groups.

To give an example, in a side event of an IGC meeting in October 2008, a WIPO pilot project in Kenya was presented. Its aim was to provide the necessary competence to the Maasai community of southern Kenya to document and digitize their traditional knowledge and folklore - a prime example for the "capacity building" programs that make up a large part of the UN's development initiatives.

During the ensuing discussion, an indigenous representative of the Samburu community of north-central Kenya voiced her concern that by documenting traditional expressions with a view to protect them, other cultural communities might be legally excluded from their rights or discriminated against. ${ }^{204}$ As the Maasai and the Samburu share a significant amount of cultural artifacts and traditional knowledge and expressions, seeking protection for Masai resources was potentially in conflict with competing claims, such as those the Samburu could make.

Hence such local entanglements multiply difference. An international process might valorize one indigenous community to the exclusion, even stigmatization, of another. Difference, in this instance, means contestation between "competing" stakeholders seeking to improve their social status. In addition to the reservations she voiced, the Samburu representative inquired whether there were plans to include her community in the documentation and preservation efforts: this would clearly mean a revaluation and strengthening of the Samburu in this context. But at the national level, changing the how indigenous communities are viewed or treated

\footnotetext{
203 There is also a pragmatic dimension of narratives, though specific social practices at times supersedes it.

204 Author's notes, October 16, 2008.
} 
is a selective and ambiguous process. The statement given by a representative of the Maasai Cultural Heritage Foundation at a WIPO panel on traditional knowledge illustrates this:

[56] The Maasai heritage in its all forms is facing serious problems and challenges. In most circumstances, the indigenous governance systems are not recognized by the Government as most of these cultural practices are considered to be primitive and do contribute to underdevelopment of the Maasai people. (Ole Kaunga 2006: 4)

When considered opportune by a government, indigenous communities may be supported. Otherwise, the stigmatization of indigenous communities is the rule rather than the exception. This is in line with the view that constructs ILCs not as autonomous social entities, but as being represented and administered by nation states, including with respect to rights involving TK, TCEs and GR. This conception was illustrated during a discussion of the scope of beneficiaries and the management of rights at IGC 17. The EU proposed adding a paragraph to the "Draft Articles on the Protection of Traditional Knowledge" that read:

[57] the competent authority shall report to WIPO each year and in a transparent way on the distribution of benefits arising from the use of TK. ${ }^{205}$

This proposal was aimed at ensuring a higher degree of control in the distribution of benefits, to ensure ILCs would profit. The Nigerian delegation countered that it was "ridiculous that people should report to WIPO", thus both stressing the principle of national sovereignty and the view that ILCs are not autonomous entities. In IGC negotiations, member states often portray themselves as speaking on behalf of "their" indigenous and local communities. As the representative of the Indigenous People (Bethechilokono) of Saint Lucia (BGC) put it, it was not clear

[58] whether those States that spoke about their respective indigenous and local communities were mandated to participate in the Committee and speak on their behalf. ${ }^{206}$

There is thus an awareness of this situations on part of ILCs in the context of IGC negotiations, that was moreover voiced by ILCs in private conversations during IGC sessions.

\footnotetext{
${ }^{205} \mathrm{WIPO} / \mathrm{GRTKF} / \mathrm{IC} / 17 / 5$.

${ }^{206} \mathrm{WIPO} / \mathrm{GRTKF} / \mathrm{IC} / 16 / 8: 37$.
} 


\section{Potential}

The "indigenous stigma" described here applies not only to individual and communal subjects, but also to material artifacts (Martínez Novo 2005), biological resources (Pilcher 1998), and traditional knowledge. The last is frequently conceived as something of potential value that is stuck in an archaic, irrational and mythical belief-system. It is seen as something pre-modern that needs to be subjected to modern science or rational calculation in order for it to be utilized and exploited (Latour 1997). Yet,

[59] [...] it is not considered so when non-Maasai have expropriated and used the same culture and used it for economic gains. The Maasai culture is a resource and it is being used by unauthorized non-Maasai for their own benefits. (Ole Kaunga 2006: 4)

In the context of the IGC, it is largely among the ILCs that one hears complaints about the devaluation of TK. One of these ILCs is Llamado de la Tierra, "an independent indigenous controlled initiative that supports and enables indigenous peoples to reframe the discussions and negotiations on intellectual property rights and traditional knowledge that are occurring in a wide range of forums, through our own perspectives and from within our own cultures". ${ }^{207}$

[60] This construction of the intrinsic value of traditional knowledge compartmentalises traditional knowledge into categories of value recognisable to non-Indigenous cultures rather than treating traditional knowledge in the interrelated and holistic manner it is regarded in Indigenous cultures. This reframing of traditional knowledge into an intellectual property framework is at the core of our dissatisfaction with the Draft. There is no mention of the role of traditional knowledge in sustaining culture and passing on information which perpetuates Indigenous identity.

The argument is that the intrinsic value of TK and its role in maintaining culture and identity is undervalued in international negotiations and specifically in WIPO's "Draft Policy Objectives and Core Principles" for the protection of TK and TCEs. Added to this aspect of the value of TK, at the indigenous panel at IGC 17 Greg Younging pointed to a more general conception of value of TK:

[61] In the early colonial period Western perspectives interpreted Indigenous Nations through the lens of Social Darwinism as subhuman and

\footnotetext{
${ }^{207}$ http://wipo.int/export/sites/www/tk/en/consultations/draft_provisions/pdf/pdf-tce-tk/ coe.pdf, accessed 20 July 2011.
} 
primitive. Consequently, despite it's immense universal value, TK was also seen to be of little or no value. ${ }^{208}$

The devaluation, or valorization, of traditional knowledge goes hand-in-hand with its perceived potential, be it for tourism, the marketing of crafts, biodiversity, or the development of pharmaceuticals. Yet, that potential needs to be revealed and to some degree separated from its aboriginal background. For tourism, a touch of "authenticity" is vital, yet it should be clean and free of conflict (Graburn 1976). For crafts, it needs to be standardized and receive a positive connotation (Chibnik 2003). For technological and agricultural innovation, it needs to be registered in databases and connected with modern knowledge (Seleti 2009). For biodiversity and potential use as medicine, the components of traditional knowledge need to be decoupled from interfering folklore and transferred to the laboratory (Hayden 2003a).

A few examples of how this stigma functions are evident in the WIPO process:

[62] Traditional Medicine is a source of prosperity proper to Oman. However this intellectual asset has so far not been fully exploited, mainly because Omani are not yet fully aware of the value of the wealth they own. (Ghafele 2005: n. pag.)

Reminiscent of the Marxist doctrine that the proletariat needs to be led by a "proletariat elite" to free themselves from oppression, this passage argues that the "wealth" hidden within archaic knowledge systems can only be capitalized on by transferring that wealth of knowledge to (scientific) processes that lie outside of these (indigenous Omani) knowledge systems. Similarly, the following alludes to the potential of traditional knowledge for pharmaceutical production:

[63] The [Egyptian] Delegation stated that traditional knowledge and its experiences were of paramount importance ... to consumers, producers and breeders in general. In addition, the Delegation stressed the importance and potential of traditional knowledge in the field of pharmaceutical production. ${ }^{209}$

It is not so much the current use and traditional practice that constitute the value of traditional knowledge, but rather the prospect of transforming it for use in "the development of scientifically acceptable products and processes" (Satish 2003). At IGC 11, the Moroccan delegation alluded in a similar vein to the potential of TK, GR, and TCEs for the development of modern technologies:

\footnotetext{
${ }^{208}$ WIPO/GRTKF/IC/17/INF/5(a):7.

${ }^{209} \mathrm{WIPO} / \mathrm{GRTKF} / \mathrm{IC} / 2 / 16: 60$.
} 
[64] In the region of North Africa, 70 per cent of wild plants had potential value in relation to medicine and biotechnology. That showed to what extent the increasing, and at the same time alarming, degree of misappropriation of GR, TK and EoF required the Committee to work for their protection. ${ }^{210}$

Such views are common in the IGC, mainly with regard to GR, which was part of the decision to establish the IGC in the first place:

[65] With the emergence of modern biotechnologies, genetic resources have assumed increasing economic, scientific and commercial value to a wide range of stakeholders. ${ }^{211}$

The "indigenous stigma", that sees something as pre-modern and irrational, needs to be removed and the knowledge associated with indigenous communities translated into the language of science and rationality. The independent ability of those indigenous communities to create and innovate is thereby also negated. This is of a part with the notion that the traditional uses of genetic resources do not reflect human intellectual activity but instead discovery, in the sense that the properties of medicinal plants first need to be discovered in order to be of value:

[66] The fact that TK is natural does not necessarily mean that there is an absence of human intellectual input. As already noted in the preceding pages, native healers in Ngwaland undergo many years of rigorous training and apprenticeship. Native healers in Ngwaland vary in their skills, competence, and knowledge. Some native healers are less knowledgeable than others. The difference in skill is often a function of their research abilities, experience, and willingness to experiment or innovate. It is therefore no coincidence that a decisive number of drugs derived from plant resources have been with the help of the most knowledgeable and innovative native healers. ${ }^{212}$

Translation is in this sense also acquisition. By transferring knowledge from a state of "uselessness", as those outside indigenous communities regard native knowledge, into something exploitable, one acquires the right to use this new knowledge. Bioprospecting, the patenting of genetic resources that have been discovered by studying traditional cultural expressions and plant use in traditional medicine (Hayden 2003) is a poignant example of this practice.

\footnotetext{
${ }^{210}$ WIPO/GRTKF/IC/11/15:12.

211 WIPO/GA/26/6:para.1.

${ }^{212}$ WIPO/GRTKF/IC/10/INF/5(d):15.
} 


\section{Rights}

From a different perspective, social struggles are fought with terminology: we are indigenous, therefore we have rights. In numerous instances, indigenous representatives in WIPO negotiations refer to the UN's "Declaration on the Rights of Indigenous Peoples" (2007) to legitimize their claims as indigenous people. This is a very frequent reference to existing international law relating to the rights of indigenous groups, for instance voiced by the representative of the BGC at IGC 16:

[67] Referring to the UN Declaration of the Rights of Indigenous Peoples, he suggested that Member States should have a copy of the UN Declaration as many had adopted it. Article 31 of the UN Declaration stated that "indigenous peoples have the right to maintain, control, protect and develop their cultural heritage, traditional knowledge and traditional cultural expressions". This issue was being discussed by the Committee and yet Member States, whilst having adopted the UN Declaration, had taken away this right from indigenous peoples. $^{213}$

The perspective is reversed in this case, such that in its positive connotation, the attribute "indigenous" is used for political and legal leverage. Existing regimes at the national and international level are referred to as legitimation for claims. The access to property, land and human rights can be established by making a link to their respective discourses.

For indigenous communities and NGOs in the UN system, various avenues exist to frame an issue. For example, UNESCO has often singled out language, or an endangered language, as a distinct marker or means for determining whether a group is indigenous and hence deserving of protection. As a matter of strategy, NGOs or ILCs in such processes would be well advised to frame their concerns and desires from the perspective of an endangered linguistic community (Toivanen 2007). WIPO, on the other hand, deals with intellectual property rights in the context of traditional knowledge, so it would be advisable to avoid the issue of language and focus on traditional knowledge and folklore. The Convention on Biological Diversity (CBD) has installed access and benefit sharing (ABS) and priorinformed consent (PIC) mechanisms that are frequently referred to by IGC participants in order to claim these rights in the context of WIPO negotiations. The statement by the NGO ICRA International (Commission Internationale pour les Droits des Peuples Indigènes) at IGC 16 provides one example:

[68] It should integrate the principles of self-determination, PIC by the communities holding TK, equitable sharing of benefits derived from

${ }^{213}$ WIPO/GRTKF/IC/16/8:37. 
biodiversity, and respect for customary indigenous rules and protocols, as well as their integration into the hierarchy of the rules contained in domestic laws. ${ }^{214}$

Different organizational settings or constellations require to frame issues accordingly, to translate oneself and one's desires into that framework. Another effort by indigenous communities is to bring human rights language in, meaning to frame and translate issues of tradition as issues of human rights and become recognized by international bodies that deal with human rights issues. That increases the pressure, in their view, on those who do not want anything to result from WIPO's IGC meetings.

The use of these discourses is not limited to references to international bodies, conventions or agreements. Global rights discourses also are entangled with national practices, since UN conventions feature in national discussions about indigenous communities as well. Indigeneity is used both as a dichotomizing marker and leverage, so terminology designed to denigrate a social group can be stood on its head and given a positive connotation. In the context of the IGC, references to indigeneity or indigenous communities then function as strategically deployable shifters. Still, to engage in such translation requires a knowledge of rights discourses, how differentiation discourses function, and be able to frame issues accordingly.

Stigma, potential, and rights all highlight differences. When perceived as a stigma, indigenous communities are deprecated, and their traditional knowledge is nullified. As long as it remains untranslated, it is treated as not being of value. In terms of rights, indigenous communities can underscore their difference in order to tap discourses that can grant them rights.

\section{Unity}

Yet, there are also perspectives that underline commonalities at the national level and externalize difference. The Declaration on the Rights of Indigenous Peoples does not contain a definition of the term "indigenous", nor does the mandate for the United Nations Permanent Forum on on Indigenous Issues. Yet, more recent and critical definitions do focus on the social and economic marginalization of groups (Lee 2006, Cadena 2007). This is problematic if the perspective a state takes is one of "national unity". The principle of national sovereignty, and domestic policy discourses, are at odds with claims of marginality due to an ascribed ethnic background or ascribed membership in a social group. Claiming indigeneity stands in opposition to national identity-building processes and struggles for na-

${ }^{214}$ WIPO/GRTKF/IC/16/8:15. 
tional unity (Anderson 1983). Again, the resistance against this political concept (e.g. by NGOs and indigenous communities in WIPO's IGC) is a vivid demonstration of how powerful it is.

One way to resolve this is to externalize difference, for example by contrasting the present with some counterpart in the past. Points of reference can be specific historical events such as national independence or, more generally, colonial phases. Colonialism is used as a shared experience, characterized in an amalgamating spirit that temporarily bridges social gaps. Susan Philips' (2000) analysis of language ideologies in Tongan courtrooms is a vivid demonstration of how linguistic regimes are used to construct a national identity that bridges social differences by referring to the past:

(...) they are invoking a relationship that establishes continuity between past and present political regimes. The distinctive features of the Tongan sister-brother relationship are viewed by Tongans as having existed prior to European contact (...) (254)

Terminology is used to construct an overarching identity based on drawing a contrast to a manner of social organization that existed in the past. The externalization of difference in the IGC context is best illustrated by a "representational complex" in which national authorities speak on behalf of "their" indigenous and local communities, thus creating a dichotomy between different actor groups that is then used as leverage in negotiations. By portraying ILCs as both represented and in accordance with national governance structures and practices, opposing parties are constructed as acting against the legitimate interests of ILCs and indirectly supporting acts of misuse and misappropriation. ${ }^{215}$ In this process, ILCs are usurped by the respective member states and subordinated to national interests, ${ }^{216}$ legitimated by an external entity constituted by colonial experience.

\footnotetext{
215 A speech by Zimbabwean president Robert Mugabe in 2000, in which he stated that "the white man is not indigenous to Africa. Africa is for Africans. Zimbabwe is for Zimbabweans," (CBC 2000) illustrates the violent potential here (Anderson 1983). Mugabe used colonialism not only as a marker in the past, but also as a political and ideological tool in the present. The "Indigenisation and Economic Empowerment Act", passed 2007 and now in force, though not consistently enforced (The Economist 2010), states that 51 per cent shares of foreign enterprises or enterprises owned by white Zimbabweans must be transferred to "indigenous Zimbabweans". Reference to the past, in this case, independence day, is made to create exclusionary practices by using the marker of indigeneity. The act defines "П] ndigenous Zimbabwean" as "any person who, before the 18th April, 1980, was disadvantaged by unfair discrimination on the grounds of his or her race, and any descendant of such person, and includes any company, association, syndicate or partnership of which indigenous Zimbabweans form the majority of the members or hold the controlling interest." (Indigenisation and Economic Empowerment Act, 1.2, 2007)
}

${ }^{216}$ Popova-Gosart 2009: 22. 
Yet colonialism is past. The time-frame in this dichotomy has changed: if colonialism is conceived of negatively, a break in time used to evaluate the past and the present, then those who lived on the land earlier become just another group of persons not judged by sociocultural realities but by an externalized entity lying in the past. The claim that every person who lived on the land before colonialism is indigenous can be heard both in diplomatic negotiations and from African delegates at the IGC. ${ }^{217}$ NGOs and indigenous communities contest this notion. There is a struggle over recognition (Honneth 1995), as the interests of traditional knowledge holders and NGOs are not identical with those expressed in national domestic or foreign policy.

Still, some ILCs continue to refer to colonialism:

[69] Consequently, the concept of TK is better understood within the context of colonialism and the irruption and truncation of the natural development of pre-colonial epistemological frameworks. In this framework, the attempt to use Western empiricism as the measuring rod for TK may be construed as a continuation of the colonial entrapment and marginalization of colonized cultures and peoples. Hence, the concept of TK is part of the legal and socio-cultural claims of indigenous peoples to shared equality, dignity, and respect with other peoples across the world. ${ }^{218}$

They also contest the argument that TK and TCEs are to be administered by the state. The monopolization of the representation of interests by nation-states is, in effect, a continuation of the politics of difference of the national level. It denies indigenous communities the right to argue their own causes and desires. At the same time, the state appropriates traditional knowledge from these communities as it claims everyone is indigenous, or that the interests of the member state and of the ILCs in that state are perfectly aligned. That makes it easy to argue that the administration of rights to traditional knowledge lies with the national government and not the indigenous communities. National unity functions as political leverage in international negotiations such as WIPO's IGC, and it is - as shown above frequently contested by ILCs.

\footnotetext{
217 Author's notes, February 2008, July 2009, December 2010.

${ }^{218}$ WIPO/GRTKF/IC/10/INF/5(d):2.
} 


\section{Justice}

To add emphasis to this argument, another dichotomy is invoked: the South versus the North, and related to it, transnational justice. Inequalities that exist between developing and developed countries are addressed in numerous UN fora (under aspects such as technology transfer, patents on essential medicines, education, and so forth). The question is whether the current UN system is fair, understood to mean in terms of equal representation of developing countries and industrialized nations (Gad 2006), and how it could be reformed.

The combination of this North/South divide with the causes raised by indigenous peoples is especially powerful, as it combines economic/moral rights with human rights. One delegate from a developing country argued during an IGC session that the current system (of intellectual property rights) for the most part benefited the industrialized nations and continues to do so, but that "now it is time for us to finally get something" (author's notes, July 2009). The statement by the Indian delegation at IGC 14 makes a similar point to invoke the notion of justice:

[70] For the first time developing countries were asking for protection of their rights and that as one NGO had pointed out, it was a very small portion of the global IP rights that developing countries were asking for, and that non-binding declarations or guiding principles or model laws would not be acceptable. The Committee was at a critical juncture where it had to be recognized that there was a need for equity, balance and justice, whether it be in IP, global equity in economy, political rights, and that it was the occasion to bring a certain semblance of proportion, equity and justice to the IP discourse. ${ }^{219}$

As illustrated, these constellations of difference are not limited to WIPO's IGC or to international negotiations. They expand to national and regional contexts and are reflected in the social realities and relationships between ILCs, the state, NGOs and a number of other actors, among them corporations interested in capitalizing on GRTKF. It would thus certainly be advisable to carry out case studies connecting the level of international negotiations with local contexts, especially with a focus on pragmatics.

In this constellation, the reference is made to past injustices as well as to current imbalances in the UN system. Speakers use a constructed national (and regional) unity to make their argument, with indigenous communities (and their traditional knowledge) at one pole of the dichotomy between North and South, only this time as witnesses to injustice, not as autonomous communal subjects.

${ }^{219}$ WIPO/GRTKF/IC/14/12:45. 
In these last two perspectives, national unity and justice, the indigenous subject is subsumed and incorporated as part of an argument against an externalized entity located either in the past (colonialism and national unity) or the present (South versus North and international justice). All the perspectives outlined in this chapter relate to the past and organize the present by creating dichotomies and differentiations.

The multiplicity of perspectives on the traditional knowledge of indigenous communities in international negotiations is in accompanied by isofunctional terminological fragments that refer to "property" (Carpenter 2009), "heritage" (Bendix 2009) or "value" (Myers 2001, 2004). These fragments are expressed in utterances and the instantiations of communicative strategies which draw on these substantive aspects. Contingencies and contested denotations (Silverstein 2004) in the terminology, for instance that used to refer to TK, TCEs and ILCs, can be better understood by taking them into account in the analysis of the IGC process. As perspectives on the substantive issues, they contribute to the specific manifestations of opening statements, referential frames and micro-edits. In the dialectical relationship between IGC participants, they play a constitutive role. They are part of the context that is needed to adequately analyze utterances. For an understanding of WIPO negotiations, the knowledge of these differing perspectives, at the level of substance, is essential, as they made a significant contribution to the communicative configuration of the IGC. By taking this level into account in the analysis of pragmatic strategies, minor utterance fragments such as "indigenous and local communities of Member States" ([50]) can be contextualized and connected to broader presuppositions about social organization, power and inequalities. 



\section{Conclusion}

In the Caribbean, we have a story: a locust comes to a barn, picks a corn, and flies away. On the next day, he comes again, picks a corn, and flies away. The next day, he comes again, picks a corn, and flies away again. The IGC is like this.

Conversation with ILC Representative at IGC 13

Juridically speaking, none of these paragraphs makes any sense. It's a mess.

Delegate of the U.S. during a Drafting Session at IGC 17

The sluggishness of IGC negotiations is astounding. Each session I attended saw delegations proclaiming they would leave the negotiating table if things didn't speed up, yet at the end of these meetings, delegates left with little to show for their efforts. True, some progress has been made since the IGC's installment in 2000, and draft texts are beginning to coalesce, but an international, legally binding, agreement is not in sight. More importantly, such an agreement would still have to be adopted by WIPO member states. It is conceivable that the outcome of these lengthy IGC deliberations might be no more than a non-binding convention or something similar to the Declaration on the Rights of Indigenous Peoples. ${ }^{220}$

On the other hand, WIPO negotiations do exert considerable influence on other processes at the national, regional and international level. Actors refer to ar-

${ }^{220}$ If widely adopted, this would be a success, as the political pressures stemming from such a declaration could benefit ILCs immensely. 
guments used in the IGC to strengthen their position, lawmakers use IGC provisions to draft national legal instruments, and bilateral agreements between member states, or between corporations and ILCs, take the issues discussed at WIPO into account.

Current scholarly research on the constituting of cultural property lacks reflection about the negotiations themselves, including about communicative practices and events. The aim of this study has been to shed light on the relationship between language and an international committee dealing with the protection of TK, TCEs and GR. Its focus has been to better understand the communicative processes involved and to gain insights into the interrelations between WIPO and other organizations or comparable situations.

The study began by surveying the debate on cultural property, and putting it in the context of broader scholarly debates. The second chapter introduced a theoretical and methodological framework drawn from linguistic anthropology, the ethnography of communication, and linguistic pragmatics. In the third chapter, the development of IGC negotiations, the range of actors and communicative events as well as the contexts in which they are situated in were delineated. This revealed the main difference between groups of member states as one between speeding up and stalling. The fourth chapter provided a detailed, case-rich, analysis of communicative strategies and patterns, while the fifth chapter sketched the diverging perspectives on TK and TCEs coupled with terminology and communicative events of the IGC.

International negotiations exist as a series of communicative events in which actors negotiate meaning with a view to achieve their particular goals. Decisions are made through a process of drafting, revising and discussing, which can involve being precise at one point and vague at another. The process involves making oral and written statements that are translated and checked for accuracy and mistakes. Differing terminology needs to be comprehended and used to take account of various fora and issues. Finally, the communicative styles vary from venue to venue and conflicting language ideologies are in place.

All these language-related aspects point to a major necessity for participants: they must have communicative competence to be able to effectively engage in negotiations. Technical competence in a given issue is insufficient. Actors also must have the linguistic competence to switch communicative styles, to micro-edit the meaning inserted into documents, and to recognize ambiguities in speech and text.

Communicative competence in this context is twofold. First, it entails the ability of an individual or a group to decipher the meaning of terminology and the arguments employed. Shifting perspectives are used to translate contextualized sociopolitical realities into specific discourses and terminology. For the purposes of analysis, these translation processes need to be retraced to grasp the meanings and implications. Second, communicative competence entails translating and trans- 
forming issues into a perspective adequate to the specific context and referential frame. Communicating one's viewpoint is less an issue of being proficient in the question at hand than it is the ability to analyze underlying perspectives and intentions - and thus being able to place oneself into what one regards as an advantageous position. Communicative competence in this sense does not only mean that what is said is appropriate in a specific situation, but also that what is said is conducive to one's intentions, and this might be described as the strategic dimension of communicative competence coupled with the reflexivity about linguistic performance, referential frames and boundary objects in the context of a communicative event.

Thus, because pragmatic strategies employed in discussions are what essentially constitute processes and outcomes, the pragmatics of speech and the performative aspects of language are of central importance for negotiations. For that reason, the role of pragmatics is significant, since both actors and their competence is contingent both on pre-structured communicative conventions and patterns and contingent on the dynamics of negotiations. The resulting contingencies and ambivalences allow participants to manipulate and exploit the communicative context according to their interests, capitalizing on instabilities caused by the emergent status of the speech community. The nascent or emergent character of the speech community is not a passing characteristic in the negotiations. Rather, because delegations see advantages in these inconsistencies, they are interested in maintaining this "state of exception" (Agamben 2005) as a basis for negotiations. In this vein, one might also term it a speech community of exception. Speeding up delegations benefit from the slow, minimal progress 221 as they can generate political, procedural and ethical pressure as a result. That leads to instabilities which need to be countered by concessions offered by the stalling delegations. Stalling delegations, on the other hand, are able to keep discussions on TK, TCEs and GR out of other fora only by making minimal progression, which is achieved by using micro-editing and other pragmatic strategies.

While all member states thus create instabilities, either by bringing direct pressure to bear or by arguing that the IGC's work does not yet suffice for an agreement, there is a simultaneous construction of a stability that prevents the negotiations from collapsing. The balance between the instabilities and stabilities that keep the committee in a state of exception is maintained primarily by employing pragmatic strategies. This is exactly why it is so important to take the study of language and language use into account when analyzing international negotiations - and not only over issues of cultural property.

The instantiations of communicative patterns and strategies analyzed in this study show how actors mediate between different perspectives and contexts, attend ${ }^{221}$ See also Bizer et al. 2010 for an economic analysis of IGC negotiations as a game of "minimal
results". 
to their interests and capitalize on situations of stability and instability. Referring to structural aspects and existing presuppositions for negotiations on the one hand, and dynamic features, contingencies and ambivalences of context, meaning and procedure on the other hand, their activities take place in, and help constitute, a discursive field ridden with contested denotations, strategic behavior, rules and conventions. It makes the IGC both chaotic and ordered at the same time.

Actors pursue strategies in international negotiations and use terms conducive to their interests. The use of terms in international negotiations is contingent on the context of the utterance, both synchronically and diachronically. To act efficiently, speakers must take pragmatic and semantic meanings and terms into account, and they have to consider the relevant referential frames and audiences. Utterances, as instantiations of communicative patterns and strategies, need to reflect on the ambiguity, vagueness and instability of a speech community in emergence. One way to use this latitude to one's advantage is by strategically deployable shifters, as they help evoke a reaction by the opposing parties. Creating such situations involves intensifying instabilities as well as generating stability. A speaker can refer back to a stable, authoritative position, while the recipient must make an effort to stabilize the situation.

The context of the IGC is one of differentiation with respect to the substantive issues being discussed. That context is one of differing, underlying conceptions and presuppositions: these are crucial for understanding the communicative processes and strategies actually adopted. They are closely coupled with terminological constellations and contested denotations of terms, which strengthens an argument for using the ethnography of communication and linguistic pragmatics as methodological and theoretical frameworks for this study. As a core concept, the speech community allows for a perspective on negotiation processes as mediating between structure and process while taking into account the diversity of participants as well as their different skill sets and levels of competence. As it does not presuppose a shared understanding, but rather argues for a partial mutual intelligibility of core issues and strategies on the part of participants, the speech community is a way of characterizing the complex constellations observed at the IGC meetings.

The pragmatic strategies and patterns of language use in the IGC can best be understood as instantiations of communicative patterns and strategies. What can be observed are not static reproductions of certain linguistic fragments or the repeated use of a fixed set of strategies, but dynamic, adapted instantiations of such patterns and strategies in context.

Two distinct types of referential frames can be distinguished in this context. The first has strong indexical meaning and assumes a high degree of shared denotationality (with respect to specific terms signifying different stages of negotiation. 
The second frame has weaker indexical meaning, refers largely to generalized statements, and its implications are, when compared to the first frame, only minor.

Referential frames are partly determined by their corresponding audiences. Here, the first referential frame refers to specific steps of action and procedures, while the second frame consists of unspecific ideological values with no implied actions. Actors shift between these two frames to make their arguments and bolster their strategies. When the first referential frame does not serve their interests or when it has been compromised by delegations in opposition, there is a switch to the second frame, and vice versa. These referential frames are also marked by a particular type of discourse: resistance to progress can be, and is, framed as caused by technical or procedural issues.

Shifting referential frames creates an instability that produces stability and instability at the same time: it therefore allows for a number of pragmatic strategies to be adopted. The use of referential frames gradually produces results, since returning to earlier stages of negotiations would imply rejecting the process. Referential frames mediate between the two stances of stalling and speeding up, where the former is primarily taken by developed countries not interested in any change of the intellectual property system and the latter is taken by delegations calling for a quick solution in the form of an international legally-binding instrument.

Referential frame shifting is also expressed in through the process of microediting. By changing small utterance fragments, actors frame texts and passages according to their perceptions and interests. Micro-edits are compensatory mechanisms that permit conflicting utterances and views to be aired while maintaining a degree of stability of the IGC as a speech community at the same time. Microedits are highly variable, strategically deployable shifters, whose meaning is mostly viable at the pragmatic, and not on the semantico-referential level.

Shifters have little to no semantico-referential meaning yet realize a pragmatic function, since the social dimension of the utterance is of what is important. As the meanings of shifters change depending on context, they cause uncertainty as to the specific pragmatic and referential meaning of an utterance. The indeterminacy and variability of shifters makes it possible to use them as part of pragmatic or useful strategies for opening statements or other communicative events in international negotiations. They enable delegations to express their perspective and signal their position to the recipients of a communicative event without having to expand on the implications and presuppositions of their utterance. Shifters not only differentiate between positions, they also map the common ground between actors who otherwise hold incommensurable views. They mediate between actors, contribute to the cohesion of the committee, and allow fundamentally different viewpoints to be expressed, part of the foundation that allows participants to negotiate at all. By indexing negotiating positions, shifters differentiate between actors and interests and refer to perspectives with regard to the substantive issues negotiated 
in the IGC. As they are shifting depending on the context, it is necessary to reflect this flexibility and resulting ambiguity on the analytical level.

Opening statements and other conversational genres heard at the IGC contain boundary objects as terms, phrases or rhetoric that bring together otherwise fundamentally different social actors. They enable actors from "intersecting social worlds" (Star and Griesemer 1989: 393) to maintain a coherent and stable identity, in this case the IGC as a speech community, even though it contains divergent, incommensurable viewpoints. The resulting speech community is flexible, as it draws from a coherence established by common factors, terms and viewpoints. Boundary objects in the context of the IGC mediate between the two main positions of stalling and speeding delegations.

Boundary objects, strategically deployable shifters, and the recipient design of opening statements are dynamic and constantly changing in response to external and internal factors. They are subject to dynamic processes influenced by the composition of actors and strategies. Boundary objects and shifters can also signal a specific stage in negotiations, as illustrated by the phrase "text-based negotiations". To some extent, this flexibility is also the result of the "state of exception" of the IGC as a speech community. If the coherence of the speech community cannot draw from an extensive history and stable communicative conventions, shifts in meanings and understandings are more likely to happen, and furthermore may even be desired by actors.

The results of this study are important for two reasons. First, they point to the need for an analytical triangulation in studying international negotiations. The ethnography of such processes needs to be complemented by micro-linguistic analyses of communicative practices and strategies in order to grasp what is happening in negotiations, at which different levels, and in relation to which referential frames. The speech community is a fruitful, expandable concept that combines these elements. Context is the third important part of the analytical triad. Macroprocesses beyond the particular setting being analyzed needs to be taken into account to understand the relations between the different levels - for instance illustrated by the perspectives of differentiation on traditional knowledge as outlined in this study.

Second, this study shows the importance of the ethnography of communication and linguistic pragmatics for the analysis of international negotiations. The pragmatics of negotiations over cultural property in this case significantly determines processes and outcomes at the international level. Without an analysis of language use in this committee, an ethnography would fail to account for important aspects and miss the dynamics, and structures, that make these negotiations possible in the first place. As pragmatic strategies are the condition for interna- 
tional negotiations to work, neglecting them at the analytical level would be a serious mistake.

For this reason I propose that such a methodological and theoretical triad of pragmatic-linguistic analysis, ethnographic contextualization and theorizing on the macro-level is needed for the study of international negotiations, in speech communities like the IGC, as well as in other international fora. 



\section{Bibliography}

Abélès, M. (2008) Rethinking NGOs: The Economy of Survival and Global Governance. Indiana Journal of Global Legal Studies 15(1):241-258.

Aberbach, J., R. Putnam, and B. Rockman (1981) Bureaucrats and Politicians in Western Democracies. Cambridge: Harvard University Press.

Adorno, T. W. (1997) Philosophische Terminologie: Zur Einleitung, Vol. 1. Frankfurt am Main: Suhrkamp.

—. (1986) Über Tradition. In Gesammelte Schriften, Vol. 10.1, ed. Rolf Tiedemann, 310-319. Frankfurt am Main: Suhrkamp.

Agamben, G. (2005) State of Exception. Chicago: University of Chicago Press.

Anderson, B. (1983) Imagined Communities: Reflections on the Origin and Spread of Nationalism. London: Verso.

Appadurai, A. (1986) The Social Life of Things: Commodities in Cultural Perspective. Cambridge: Cambridge University Press.

Audi, A. (2007) A Semiotics of Cultural Property Argument. International Journal of Cultural Property 14(02):131-156.

Austin, J. L. (1962) How to Do Things with Words. Glasgow: Oxford University Press.

Bakhtin, M. (1983) The Dialogic Imagination, eds. Michael Holquist, trans. Caryl Emerson, and Michael Holquist. Austin: University of Texas Press. 
- (2007) The Problem of Speech Genres. In Speech Genres and Other Late Essays, eds. Caryl Emerson, and Michael Holquist, trans. Vern W. McGee, 60102. Austin: University of Texas Press.

Barber, P., and W. Lem (2004) Commodities, Capitalism and Globalization. Special Journal Issue of Anthropologica 40(2).

Barth, F. (1969) Ethnic Groups and Boundaries. the Social Organization of Culture Difference. Oslo: Universitetsforlaget.

Bauman, R. (1977) Verbal Art as Performance. Prospect Heights: Waveland Press.

- (1983) Let Your Words Be Few: Symbolism of Speaking and Silence Among Seventeenth-Century Quakers. Cambridge: Cambridge University Press.

Bauman, R., and C. Briggs (1990) Poetics and Performances as Critical Perspectives on Language and Social Life. Annual Review of Anthropology 19(1):59-88.

- (2003) Voices of Modernity. Language Ideologies and the Politics of Inequality. Cambridge: Cambridge University Press.

Bauman, R., and J. Sherzer, eds. (1989) Explorations in the Ethnography of Speaking. Cambridge: Cambridge University Press.

Bausinger, H. (1969) Kritik der Tradition. Anmerkungen zur Situation der Volkskunde. Zeitschrift für Volkskunde 65:232-250.

—. (1991) Zum Begriff des Folklorismus. In Der blinde Hund: Anmerkungen zur Alltagskultur, eds. E. Frahm, W. Alber, 92-103. Tübingen: Verlag Schwäbisches Tagblatt.

Ben-Amos, D. (1984) The Seven Strands of Tradition: Varieties in Its Meaning in American Foklore Studies. Journal of Folklore Research 21(2):97-132.

Bendix, R. (1995) Amerikanische Folkloristik: Eine Einführung. Berlin: Reimer.

- (1997) In Search of Authenticity: The Formation of Folklore Studies. Madison: University of Wisconsin Press.

_. (2002) Capitalizing on Memories Past, Present and Future: Observations on the Intertwining of Tourism and Narration. Anthropological Theory 2(4):469-487.

- (2007) Kulturelles Erbe zwischen Wirtschaft und Politik: Ein Ausblick. In Prädikat Heritage. Wertschöpfungen aus kulturellen Ressourcen, eds. Dorothee Hemme, Markus Tauschek, and Regina Bendix, 337-355. Münster: Lit.

- (2009a) Heritage between Economy and Politics: An Assessment from the Perspective of Cultural Anthropology. In Intangible Heritage, eds. Laurajane Smith, and Natsuko Akagawa, 253-269. London: Routledge. . (2009b) Inheritances: Possession, Ownership and Responsibility. Traditiones 38(2):181-199. 
Bendix, R., and V. Hafstein, eds. (2009) Culture and Property. Special issue of Ethnologia Europaea 39:2.

Bendix, R., K. Bizer, and S. Groth, eds. (2010) Die Konstituierung von Cultural Property: Forschungsperspektiven. Göttingen: Universitätsverlag Göttingen.

Bendix, R., A. Eggert, and A. Peselmann, eds. (2012) Heritage Regimes and the State. Göttingen: Universitätsverlag Göttingen.

Benjamin, W. (2008) Das Kunstwerk im Zeitalter seiner technischen Reproduzierbarkeit. Frankfurt am Main: Suhrkamp.

Bhatia, V., J. Engberg and M. Gotti, eds. (2005) Vagueness in Normative Texts. Bern: Peter Lang.

Bhatia, V., C. Candlin, and P. Evangelisti Allori, eds. (2008) Language, Culture and the Law: The Formulation of Legal Concepts across Systems and Cultures. Bern: Peter Lang.

Binsbergen, W., and P. Geschiere, eds. (2005). Commodification: Things, Agency and Identities. Münster: Lit.

Bizer, K., Z. Gubaydullina, and M. Lankau (2010) Die verborgene Effektivität minimaler Resultate in internationalen Verhandlungen: Der Fall der WIPO. In Die Konstituierung von Cultural Property: Forschungsperspektiven, eds. R. Bendix, K. Bizer, and S. Groth, 197-216. Göttingen: Universitätsverlag Göttingen, 2010.

Blakeney, M. (1999) Intellectual Property in the Dreamtime - Protecting the Cultural Creativity of Indigenous Peoples. Paper presented at the Oxford Intellectual Property Research Centre. http://www.oiprc.ox.ac.uk/EJWP1199.pdf, last accessed 30.07.2011.

Blommaert, J., and M. Meeuwis (1998) A Monolectial View on Code-Switching: Layered Code-Switching Among Zairians in Belgium. In Code-Switching in Conversation: Language, Interaction and Identity, ed. Peter Auer, 76-100. London: Routledge.

Brenneis, D. (1984) Straight Talk and Sweet Talk: Political Discourse in an Occasionally Egalitarian Community. In Dangerous Words: Language and Politics in the Pacific, eds. Donald F. Brenneis, and Fred R. Myers, 69-84. New York: New York University Press.

- (1988) Language and Disputing. Annual Review of Anthropology 17:221237.

(1996) Speaking Law, Making Difference. PoLAR: Political \& Legal Anthropology Review 19(1):117-123. 
- (2006) Reforming Promise. In Documents: Artifacts of Modern Knowledge, ed. Annelise Riles, 41-70. Ann Arbor: University of Michigan Press.

- (2009) Performing Passions: Aesthetics and Politics in an Occasionally Egalitarian Community. American Ethnologist 14(2):236-250.

Brenneis, D., and F. Myers (1984) Dangerous Words: Language and Politics in the Pacific. New York: New York University Press.

Briggs, C. (1986) Learning How to Ask: A Sociolinguistic Appraisal of the Role of the Interview in Social Science Research. Cambridge: Cambridge University Press.

- (1988) Competence in Performance: The Creativity of Tradition in Mexicano Verbal Art. Philadelphia: University of Pennsylvania Press.

— . (1996) The Politics of Discursive Authority in Research on the "Invention of Tradition". Cultural Anthropology 11(4):435-469.

. (2005) Communicability, Racial Discourse, and Disease. Annual Review of Anthropology 34:269-291.

- (2007) Anthropology, Interviewing, and Communicability in Contemporary Society. Current Anthropology 48(4):551-580.

- (2012) What We Should Have Learned from Américo Paredes: The Politics of Communicability and the Making of Folkloristics. Journal of American Folklore 125(495):91-110.

Briggs, C., and R. Bauman (1992) Genre, Intertextuality, and Social Power. Journal of Linguistic Anthropology 2(2):131-172.

Briggs, C., and C. Mantini-Briggs (2003) Stories in the Time of Cholera: Racial Profiling during a Medical Nightmare. Berkeley: University of California Press.

Brown, M. (2003). Who Owns Native Culture? Cambridge: Harvard University Press.

- (2005) Heritage Trouble: Recent Work on the Protection of Intangible Cultural Property. International Journal of Cultural Property 12:40-61.

Brumann, C. (1999) Writing for Culture: Why a Successful Concept Should Not Be Discarded. Current Anthropology 40(S1):1-27.

Burke, L., T. Crowley, and A. Girvin (2000) The Routledge Language and Cultural Theory Reader. London: Routledge.

Cadena, M., and O. Starn (2007) Indigenous Experience Today. Oxford: Berg.

Carnap, R. (1951) The Nature and Application of Inductive Logic. Chicago: University of Chicago Press. 
Carneiro da Cunha, M. (2009) "Culture" and Culture: Traditional Knowledge and Intellectual Rights. Chicago: Prickly Paradigm Press.

Carpenter, K., S. Katyal, and A. Riley (2009) In Defense Of Property. Yale Law Journal 118:1022-1125.

Carston, R. (2004) Truth-Conditional Content and Conversational Implicature. In The Semantics/Pragmatics Distinction, ed. C. Bianchi, 18-48. Stanford: CSLI.

Castiglione, D., and C. Longman (2007) The Language Question in Europe and Diverse Societies: Political, Legal and Social Perspectives. Oxford: Hart Publishing.

Chibnik, M. (2003) Crafting Tradition: The Making and Marketing of Oaxacan Wood Carvings. Austin: University of Texas Press.

Chomsky, N. (1965) Aspects of the Theory of Syntax. Cambridge: MIT Press.

CIEL - Center for International Environmental Law (2008) The Gap Between Indigenous Peoples' Demands and WIPO's Framework on Traditional Knowledge. Paper distributed at the Twelfth Session of the IGC.

Coombe, R. (2008) The Cultural Life of Intellectual Properties: Authorship, Appropriation and the Law. Durham: Duke University Press.

Davis, S. (1991) Pragmatics: A Reader. Oxford: Oxford University Press.

Dorson, R. (1976) Folklore and Fakelore: Essays Toward a Discipline of Folk Studies. Cambridge: Harvard University Press.

Dundes-Renteln, A. (1988) Relativism and the Search for Human Rights. American Anthropologist 90(1):56-72.

Duranti, A. (1988) Intentions, Language, and Social Action in a Samoan Context. Journal of Pragmatics 12: 13-33.

- (1993) Truth \& Intentionality: An Ethnographic Critique. Cultural Anthropology 8(2):214- 245.

—. (1997) Linguistic Anthropology. Cambridge: Cambridge University Press.

- (1999b) Intentionality. Journal of Linguistic Anthropology 9(1-2):134-136.

- (1999c) Relativity. Journal of Linguistic Anthropology 9(1-2):220-222.

_. (2001) Linguistic Anthropology: A Reader. Malden: Blackwell.

- (2002) Linguistic Anthropology. In International Encyclopedia of the Social and Behavioral Sciences, 8899-8906. Oxford: Elsevier.

- (2003) Language as Culture in U.S. Anthropology. Current Anthropology 44(3):323-347.

_. (2004) A Companion to Linguistic Anthropology. Malden: Blackwell. 
. (2006) The Social Ontology of Intentions. Discourse Studies 8 (1):31-40.

Duranti, A., ed. (1999a) Language Matters in Anthropology: A Lexicon for the New Millennium. Special Issue of the Journal of Linguistic Anthropology, 9 (12).

Dutfield, G. (2003) Intellectual Property Rights \& the Life Science Industries: A Twentieth Century History. London: Ashgate.

- (2004) Intellectual Property, Biogenetic Resources and Traditional Knowledge. London: Earthscan.

Dutfield, G., and U. Suthersanen (2008) Global Intellectual Property Law. Cheltenham: Edward Elgar.

Errington, J. (2001) Ideology. In Key Terms in Language and Culture, ed. Alessandro Duranti, 110-112.

Fenelon, J., and T. Hall (2008) Indigenous Movements and Globalization: What is Different? What is the Same? Globalizations 5:1-11.

Fisher, W. (1997) Doing Good? The Politics and Antipolitics of NGO Practices. Annual Review of Anthropology 26:439-464.

Fox, B. (2008) Dynamics of Discourse. In Handbook of Interpersonal Communication, eds. G. Antos, E. Ventola, and T. Weber, 225-284. Berlin: Mouton De Gruyter.

Fabian, J. (1977) Taxonomy and Ideology: On the Boundaries of ConceptClassification. In Linguistics and Anthropology: In Honor of C.F. Voegelin, eds. M. Dale Kinkade, Kenneth L. Hale, and Oswald Werner, 183-197. Lisse: Peter de Ridder Press.

- (1983) Time and the Other: How Anthropology Makes Its Object. New York: Columbia University Press.

Gad, M. (2006) Representational Fairness in WTO Rule Making: Negotiating, Implementing and Disputing the TRIPS Pharmaceutical-Related Provisions. London: British Institute of International and Comparative Law.

Gal, S. (1998) Multiplicity and Contestation Among Linguistic Ideologies. In Language Ideologies: Practice and Theory, eds. Kathryn Woolard, and Bambi Schieffelin, 317-331. Oxford: Oxford University Press.

- (2005) Language Ideologies Compared. Journal of Linguistic Anthropology, 15(1), 23-37.

_- (2008a) Language and Political Space. In Language and Space, eds. P. Auer, and J. Schmidt, 33-50. Berlin: Mouton de Gruyter. 
. (2008b) Perspective and the Politics of Representation. In Beyond Yellow English. Toward a Linguistic Anthropology of Asian Pacific America, eds. A. Reyes, and A. Lo, 325-330. Oxford: Oxford University Press.

Gal, S., and K. Woolard, eds. (2001). Languages and Publics: The Making of Authority. Manchester: St. Jerome's Press.

Garsten, C., and M. Lindh de Montoya (2008) Transparency In A New Global Order: Unveiling Organizational Visions. Cheltenham: Edward Elgar.

Geertz, C. (1984) Distinguished Lecture: Anti Anti-Relativism. American Anthropologist 86:263- 278.

Giannoni, D. (2005) 'Any dispute shall be settled by arbitration': A Study of Vagueness in International Model Arbitration Clauses. In Vagueness in Normative Texts, eds. Bhatia, Vijay K., Jan Engberg, Maurizio Gotti, and Dorothee Heller, 437-467. Bern: Peter Lang.

Goffman, E. (1959) The Presentation of Self in Everyday Life. Edingurgh: University of Edinburgh Social Sciences Research Centre.

- (1974) Frame Analysis: an Essay on the Organization of Experience. London: Harper and Row.

Goodwin, C. (1994) Professional Vision. American Anthropologist 96(3):606-633.

Glassie, H. (1995) Tradition. Journal of American Folklore 108(430):395-412.

Graber, C. (2007) Traditional Cultural Expressions in a Matrix of Copyright, Cultural Diversity and Human Rights. In New Directions in Copyright Law, ed. Fiona Macmillan, 45-71. Cheltenham: Edward Elgar.

Graburn, N. (1976) Ethnic and Tourist Arts: Cultural Expressions from the Fourth World. Berkeley: University of Berkeley Press.

- (2001) Learning to Consume: What is Heritage and When is it Traditional? In Consuming Tradition, Manufacturing Heritage: Global Norms and Urban Forms in the Age of Tourism, ed. Nezar AlSayyad, 68-89. London: Routledge.

Gumperz, J. (1972) The Speech Community. In Language and Social Context, ed. P. Giglioli, 219-31. London: Penguin.

Gumperz, J., and D. Hymes (1954) Directions in Sociolinguistics: The Ethnography of Communication. Oxford: Blackwell.

Gumperz, J., and J. Cook-Gumperz (2008) Studying Language, Culture, and Society: Sociolinguistics or Linguistic Anthropology?. Journal of Sociolinguistics 12(4):532-545. 
Groth, S. (2009) Tradition und Wert: Die Weltorganisation für Geistiges Eigentum, Cultural Property und die Vorzeitigkeit von Tradition. Kulturen 2/2009:33-39. . (2010a) Perspectives of Differentiation: Negotiating Traditional Knowledge on the International Level. Journal of Ethnology and Folkloristics 4(1):724.

. (2010b) Perspektiven der Differenzierung: Multiple Ausdeutungen von traditionellem Wissen indigener Gemeinschaften in WIPO-Verhandlungen. In Die Konstituierung von Cultural Property: Forschungsperspektiven, eds. R. Bendix, K. Bizer, S. Groth, 177-195. Göttingen: Universitätsverlag Göttingen.

Hafstein, V. (2004) The Politics of Origins: Collective Creation Revisited. Journal of American Folklore 117(465):300-315.

- (2008) Claiming Culture: Intangible Heritage Inc., FolkloreC, Traditional Knowledge ${ }^{\mathrm{TM}}$. In Prädikat Heritage. Wertschöpfungen aus kulturellen Ressourcen, eds. D. Hemme, M. Tauschek, and R. Bendix, 75-100. Münster: Lit.

- (2009) Collectivity by Culture Squared: Cultural Heritage in Nordic Spaces. Arv - Nordic Yearbook of Folklore 65:11-23.

- (2012) Cultural Heritage. In A Companion to Folklore, eds. R. Bendix, and G. Hasan-Rokem, 500-519. Malden: Wiley-Blackwell.

Halewood, M. (1999) Indigenous and Local Knowledge in International Law: A Preface to Sui Generis Intellectual Property Protection. McGill Law Journal 44:953-996.

Hall, S., and S. Maharaj (2001) Annotations: Modernity and Difference. London: Institute of International Visual Arts (INIVA).

Handler, R., and J. Linnekin (1984) Tradition, Genuine or Spurious. The Journal of American Folklore 97(385):273-290.

Hann, C. (1998) Introduction: The Embeddedness of Property. In Property Relations: Renewing the Anthropological Tradition, ed. Chris M. Hann, 1-47. Cambridge: Cambridge University Press.

Hayden, C. (2003a) From Market to Market: Bioprospecting's Idioms of Inclusion. American Ethnologist 30(3):359-371.

- (2003b) When Nature Goes Public: The Making and Unmaking of Bioprospecting in Mexico. Princeton: Princeton University Press.

- (2005) Bioprospecting's Representational Dilemma. Science as Culture 14(2):185-200.

. (2007) A Generic Solution? Pharmaceuticals and the Politics of the Similar in Mexico. Current Anthropology 48(4):475-495.

Hegel, G. (2006[1807]) Phänomenologie des Geistes. Hamburg: Meiner.

Hilf, M. (1973) Die Auslegung mehrsprachiger Verträge. Berlin: Springer. 
Hobsbawm, Eric, and Terence Ranger (1992) The Invention of Tradition. Cambridge: Cambridge University Press.

Horkheimer, M. (1947). Eclipse of Reason. New York: Oxford University Press.

Hutton, C. (2009) Language, Meaning and the Law. Edingburgh: Edingburgh University Press.

Hymes, D. (1962) The Ethnography of Speaking. In Anthropology and Human Behavior, eds. T. Gladwin and W. Sturtevant, 13-53. Washington: The Anthropology Society of Washington.

— . (1964) The Ethnography of Communication. Washington: American Anthropological Association.

—_. (1971) On Communicative Competence. Philadelphia: University of Pennsylvania Press.

- (1975) Folklore's Nature and the Sun's Myth. Journal of American Folklore 88(350):345-369.

- (1979) Soziolinguistik: Zur Ethnographie der Kommunikation. Frankfurt: Suhrkamp.

- (1986) Foundations of Sociolinguistics: An Ethnographic Approach. Philadelphia: University of Pennsylvania Press.

Irvine, J., and S. Gal (2000) Language Ideology and Linguistic Differentiation. In Regimes of Language: Ideologies, Polities and Identities, eds. P. Kroskrity, 3584. Santa Fe: School of American Research Press

Jacobs, S. (2007) Edward Shils' Theory of Tradition. Philosophy of the Social Sciences 37(2):139-162.

Jakobson, R. (1960) Linguistics and Poetics. In Style in Language, ed. T. Sebeok, 350-377. Cambridge: MIT Press.

- (1971) Shifters, Verbal Categories and the Russian Verb. In Selected Writings II: Word and Language, ed. R. Jakobson, 130-147. The Hague: Mouton.

Janke, T. (2003) Minding Culture: Case Studies on Intellectual Property and Traditional Cultural Expression. Geneva: World Intellectual Property Organization.

Jansen, B. (1999) Die Verwendung der EU Amtssprache in Internationalen Abkommen. EuZW. Europäische Zeitung für Wirtschaftsrecht 1:1-32.

Johler, R. (2009) Kulturelles Erben: Eine europäische Zustandsbeschreibung. In Erb.gut? Kulturelles Erbe zwischen Wissenschaft und Gesellschaft, eds. Karl C. Berger, Margot Schindler, and Ingo Schneider, 35-48. Wien: Verein für Volkskunde.

Kamusella, T. (2009) The Politics of Language and Nationalism in Central Europe. Houndmills: Palgrave Macmillan. 
Kapchan, D. (1995) Performance. Journal of American Folklore 108(430): 479508.

Kasten, E. (2002a) Cultural Heritage: Property of Individuals, Collectivities or Humankind? Max Planck Institute for Social Anthropology Working Papers 39:1-13.

Kasten, E., ed. (2002b) People and the Land: Pathways to Reform in Post-Soviet Siberia. Berlin: Dietrich Reimer Verlag.

- (2004) Properties of Culture, Culture as Property: Pathways to Reform in Post-Soviet Siberia. Berlin: Dietrich Reimer Verlag.

Kay, P., and W. Kempton (1984) What is the Sapir-Whorf-Hypothesis? American Anthropologist 86(1):65-79.

Keating, E. (2001) The Ethnography of Communication. In Handbook of Ethnography, eds. P. Atkinson, A. Coffey, S. Delamont, J. Lofland, and L. Lofland, 285-301. London: Sage.

Kirshenblatt-Gimblett, Barbara (1993) Theorizing Heritage. Ethnomusicology 39(3):367-380.

- (1998) Destination Culture: Tourism, Museums, and Heritage. Berkeley: University of California Press.

Kojève, Alexandre (1969) Introduction to the Reading of Hegel: Lectures on the Phenomenology of Spirit. Ithaca: Cornell University Press.

Korta, K., and J. Perry (2011) Critical Pragmatics: An Inquiry into Reference and Communication. Cambridge: Cambridge University Press.

Kroskrity, P. (2004) Language Ideologies. In A Companion to Linguistic Anthropology, ed. A Duranti, 496-517. Malden: Blackwell.

Kuutma, K. (2009a). Who Owns Our Songs? Authority of Heritage and Resources for Restitution. Ethnologia Europaea 39(2):26-40.

- (2009b) Cultural Heritage: An Introduction to Entanglements of Knowledge, Politics. Journal of Ethnology and Folkloristics 3(2):5-12.

Latour, B. (1997). Nous n'Avons Jamais Été Modernes. Paris: La Découverte.

Lee, R. (2006) Twenty-first Century Indigenism. Anthropological Theory 6(4):455-479.

Levinson, S. (1983) Pragmatics. Cambridge: Cambridge University Press.

Luttermann, C. (1999) Rechtssprachenvergleich in der Europäischen Union: Ein Lehrbuchfall. EuZW: Europäische Zeitung für Wirtschaftsrecht 13:401-416. 
Mar-Molinero, C. (2000) The Politics of Language in the Spanish-Speaking World: From Colonisation to Globalisation. London: Routledge.

Marceau, C. (2007) The Ethics of Collecting: Universality Questioned. Museum International 59(3):80-87.

Marinova, D., and M. Raven (2006) Indigenous Knowledge and Intellectual Property: A Sustainability Agenda. Journal of Economic Surveys 20(4):587-605.

Marks, J. (2009) Why I Am Not a Scientist: Anthropology and Modern Knowledge. Berkeley: University of California Press.

Martin, L. (1986) "Eskimo Words for Snow"': A Case Study in the Genesis and Decay of an Anthropological Example. American Anthropologist 88(2):418-423.

Martínez Novo, C. (2005) Who Defines Indigenous? Identities, Development, Intellectuals, and the State in Northern Mexico. New Brunswick: Rutgers University Press.

Merry, S. (2006a) Anthropology and International Law. Annual Review of Anthropology 35:99- 116.

- (2006b) Transnational Human Rights and Local Activism: Mapping the Middle. American Anthropologist 18(1):38-51.

Mertz, E. (2007) The Language of Law School: Learning to “"Think Like a Lawyer". Oxford: Oxford University Press.

Mey, J. L. (1993). Pragmatics. An Introduction. Oxford: Blackwell.

Mgbeoji, I. (2006) Global Biopiracy: Patents, Plants and Indigenous Knowledge. Ithaca, NY: Cornell University Press.

Morgan, M. (1999) Community. Journal of Linguistic Anthropology 9(1-2):36-38.

Morris, C. (1938) Foundations of the Theory of Signs. Chicago: University of Chicago Press

Münch, I. (2002) Sprechen und Schweigen im Recht. NJW: Neue Juristische Wochenschrift 28: 1995-2001.

Myers, F. (2001) The Empire of Things: Regimes of Value and Material Culture. Santa Fe: School of American Research Press.

(2004) Ontologies of the Image and Economies of Exchange. American Ethnologist 31(1):5-20.

Myers-Scotton, C. (1998) Codes and Consequences: Choosing Linguistic Varieties. Oxford: Oxford University Press. 
Nadasdy, P. (2003) Hunters and Bureaucrats: Power, Knowledge, and AboriginalState Relations in the Southwest Yukon. Vancouver: University of British Columbia Press.

Nic Craith, M. (2007) Language, Power and Identity Politics. Houndmills: Palgrave Macmillan.

Noyes, D. (2003) Fire in the Plaça: Catalan Festival Politics After Franco. Philadelphia: University of Pennsylvania Press.

- (2006) The Judgement of Solomon: Global Protections for Tradition and the Problem of Community Ownership. Cultural Analysis 5:27-56.

- (2007) Voice in the Provinces: Submission, Recognition, and the Making of Heritage. In Prädikat Heritage: Wertschöpfungen aus kultureller Ressourcen, eds. D. Hemme, M. Tauschek and R. Bendix, 33-52. Münster: Lit.

- (2009) Tradition: Three Traditions. Journal of Folklore Research 46(3):233-268.

- (2010) Traditional Culture: How Does It Work? Concepts and Institutions in Cultural Property Working Paper Series 1/2010:1-5.

Ole Kaunga, J. (2006) Experiences from Kenya. Background Information Prepared for the WIPO Panel on Indigenous and Local Communities' Concerns and Experiences in Promoting, Sustaining and Safeguarding their Traditional Knowledge, Traditional Cultural Expressions and Genetic Resources, Geneva. http://www.kipo.ke.wipo.net/export/sites/www/tk/en/ngoparticipation/ind_ loc_com/pdf/ole-kaunga.pdf, last accessed 02.06.2011.

Patrick, P. (2008) The Speech Community. In Handbook of Language Variation and Change, eds. J. Chambers, P. Trudgill and N. Schilling-Estes, 573-597. Oxford: Blackwell.

Pfaff-Czarnecka, J. (2005) Das Lokale als Ressource im entgrenzten Wettbewerb: Das Verhandeln kollektiver Repräsentationen im Nepal-Himalaya. Zeitschrift für Soziologie Sonderheft Weltgesellschaft: 479-499.

Philips, S. (2000) Constructing a Tongan Nation-State through Language Ideology in the Courtroom. In Regimes of Language: Ideologies, Polities and Identities, ed. P. Kroskrity, 229-258. Santa Fe: School of Amercian Research Press.

Pilcher, J. (1998) Que Vivan los tamales!: Food and the Making of Mexican Identity. Albuquerque: University of New Mexico Press.

Popova-Gosart, U. (2009) Traditional Knowledge \& Indigenous Peoples. Geneva: World Intellectual Property Organization.

Povinelli, E. (2002) The Cunning of Recognition: Indigenous Alterities and the Making of Australian Multiculturalism. Durham: Duke University Press. 
Pratt, M. (2007) Afterword: Indigeneity Today. In Indigenous Experience Today, eds. M. de la Cadena and O. Starn, 397-404. Oxford: Berg.

Pressman, J. (1994) Pragmatics in the Late Twentieth Century: Countering Recent Historiographic Neglect. Pragmatics 4(4):461-489.

Putnam, R. (1988) Diplomacy and Domestic Policy: The Logic of Two-Level Games. - International Organization 42(3):427-460.

Rampton, B. (2007) Neo-Hymesian linguistic ethnography in the United Kingdom. Journal of Sociolinguistics 11(5):584-607.

Raven, M. (2006) Protocols \& ABS: Recognising Indigenous Rights to Knowledge in Australian Bureaucratic Organisations. Indigenous Law Bulletin 39:1-6.

Richards, A., and A. Kuper, eds. (1971) Councils in Action. New York: Columbia University Press.

Richland, J. (2008) Arguing with Tradition: The Language of Law in Hopi Tribal Court. Chicago: University of Chicago Press.

Rikoon, J. (2004) On the Politics of the Politics of Origins. Social (In)Justice and the International Agenda on Intellectual Property, Traditional Knowledge, and Folklore. Journal of American Folklore 117(465):325-336.

Riles, A. (1999) Models and Documents: Artefacts of Modern International Knowledge. International and Comparative Law Quarterly 48:805-825. (2006) Documents: Artifacts of Modern Knowledge. Ann Arbor: University of Michigan Press.

Sacks, H. (1972a) On the Analyzability of Stories by Children. In Directions in Sociolinguistics: the Ethnography of Communication, eds. J. Gumperz, and D. Hymes. New York: Rinehart \& Winston.

- (1972b) An Initial Investigation of the Usability of Conversational Data for Doing Sociology. In Studies in Social Interaction, ed. D. Sudnow. New York: Free Press.

Sacks, H., E. Schegloff, and G. Jefferson (1974) A Simplest Systematics for the Organisation of Turn-Taking for Conversation. Language 50:696-735.

Sahlins, M. (1976) Culture and Practical Reason. Chicago: University of Chicago Press.

Salzmann, Z. (1993) Language, Culture, and Society: An Introduction to Linguistic Anthropology. Boulder: Westview. 
Satish, N. (2003) Rediscovering Traditional Knowledge: A Case Study of Neem. International Journal of Information Technology and Management 2(3):184-196.

Saville-Troike, M. (2003) The Ethnography of Communication: An Introduction. Oxford: Blackwell.

Schiffman, H. (1996) Linguistic Culture and Language Policy. London: Routledge.

Schuller, M. (2009) Gluing Globalization: NGOs as Intermediaries in Haiti. PoLAR: Political and Legal Anthropology Review 32(1):84-104.

Schwartzman, H. (1989) The Meeting: Gatherings in Organizations and Communities. New York: Plenum Press.

Seleti, Y. (2009) Promoting Access to Traditional African Medicine, Managing the IPR: The Case of South Africa. Presentation at the Conference on Intellectual Property and Public Policy Issues, http://www.wipo.int/export/sites/www/ meetings/en/2009/ip_gc_ge/presentations/seleti.pdf, last accessed 13.07.2011.

Shand, P. (2002) Scenes from the Colonial Catwalk: Cultural Appropriation, Intellectual Property Rights, and Fashion. Cultural Analysis 3:47-88.

Sherzer, J. (1983) Kuna Ways of Speaking: An Ethnographic Perspective. Austin: University of Texas Press

Shils, E. (1981) Tradition. Chicago: University of Chicago Press.

Shore, C. (2000) Building Europe. The Cultural Politics of European Integration, London: Routledge.

Silverman, H., and D. Ruggles (2007) Cultural Heritage and Human Rights. New York: Springer.

Silverstein, M. (1976) Shifters, Linguistic Categories, and Cultural Description. In Meaning in Anthropology, eds. Keith H. Basso, and Henry A. Selby, 11-55. Santa Fe: School of American Research Press.

- (1996) Monoglot 'standard' in America: Standardization and metaphors of linguistic hegemony. In The Matrix of Language: Contemporary Linguistic Anthropology, eds. D. Brenneis, and R. Macaulay, 284-306. Boulder: Westview Press.

- (1998) Contemporary Transformations of Local Linguistic Communities. Annual Review of Anthropology 27:401-426

. (2001) The Limits of Awareness. In Linguistic Anthropology: A Reader, ed. A. Duranti, 382-401. Malden: Blackwell.

(2003) Talking Politics: The Substance of Style from Abe to "W". Chicago: Prickly Paradigm Press. 
- (2004) "Cultural" Concepts and the Language-Culture Nexus. Current Anthropology 45(5): 621-652.

Skrydstrup, M. (2012) Cultural Property. In A Companion to Folklore, eds. R. Bendix, and G. Hasan-Rokem, 520-536. Malden: Wiley-Blackwell.

Spiro, M. (1986) Cultural Relativism and the Future of Anthropology. Cultural Anthropology 1(3):259-286.

Star, S., and J. Griesemer (1989) Institutional Ecology, 'Translations' and Boundary Objects: Amateurs and Professionals in Berkeley's Museum of Vertebrate Zoology, 1907-39. Social Studies of Science 19 (3):387-420

Strang, V. (2006) A Happy Coincidence? Symbiosis and Synthesis in Anthropological and Indigenous Knowledges. Current Anthropology 47(6):981-1001.

Tauschek, M. (2010) Wertschöpfung aus Tradition: Der Karneval von Binche und die Konstituierung kulturellen Erbes. Berlin: Lit.

. (2011) Reflections on the Metacultural Nature of Intangible Cultural Heritage. Journal of Ethnology and Folkloristics 5(2):49-64.

The Economist (2010) The "Indigenisation" of Zimbabwe: Foreigners and Local Whites Out. http://www.economist.com/world/middle-east/displaystory.cfm ?story_id=15955482, last accessed 10.05.2011.

Thedvall, R. (2006) Eurocrats at Work. Negotiating Transparency in Postnational Employment Policy. Stockholm Studies in Social Anthropology, 58. Stockholm: Almqvist and Wiksell International.

Toivanen, R. (2007) Linguistic Diversity and the Paradox of Rights Discourse. In The Language Question in Europe and Diverse Societies: Political, Legal and Social Perspectives, eds. Dario Castiglione und Chris Longman, 101-121. Oxford: Hart.

Triebel, V., and S. Balthasar (2004) Auslegung Englischer Vertragstexte unter Deutschen Vertragsstatut: Fallstricke des Art. 32 I Nr. 1 EGBGB. NJW. Neue Juristische Wochenschrift 31:2189-2196.

Trouillot, M. (2003) Global Transformations: Anthropology and the Modern World. Houndmills: Palgrave Macmillan.

Urciuoli, B. (2000) Strategically Deployable Shifters in College Marketing, or Just What Do They Mean "Skills" and "Leadership" and "Multiculturalism"? Language and Culture Symposium 6 Working Paper, http://language-culture.bing hamton.edu/symposia/6, last accessed 15.06.2011.

Verschueren, J. (1985) Review Article of Stephen Levinson's 'Pragmatics', Journal of Linguistics 21(2):459-470 
Wetzel, L. (2011) Types and Tokens, The Stanford Encyclopedia of Philosophy, eds. Zalta, E., http://plato.stanford.edu/archives/spr2011/entries/types-tokens last accessed 10.07.2012.

Williams, C. (2005) Vagueness in Legal Texts: Is There a Future for Shall? In Vagueness in Normative Texts, eds. Bhatia, Vijay K., Jan Engberg, Maurizio Gotti, and Dorothee Heller, 201-225. Bern: Peter Lang.

Wittgenstein, Ludwig (1963) Tractatus Logico-Philosophicus. Frankfurt am Main: Suhrkamp.

Woolard, K. (2004) Codeswitching. In A Companion to Linguistic Anthropology, ed. A. Duranti, 73-94. Malden: Blackwell.

Yankah, K. (1995) Speaking for the Chief. Okyeame and the Politics of Akan Royal Oratory. Bloomington: Indiana University Press.

Yarrow, T. (2008) Negotiating Difference: Discourses of Indigenous Knowledge and Development in Ghana. PoLAR: Political and Legal Anthropology Review 31(2):224-242. 


\begin{abstract}
Communicative interactions in international negotiations on cultural property not only provide information about the emergence and proliferation of arguments, rhetorics, and registers, but also permit valuable insights into actors' positions, strategies and alliances. They significantly influence local and national practices and views related to cultural property debates. What can be gained from a deep analysis of the communicative patterns and strategies that actors engage in - the entailing text and talk of negotiations - is a better understanding of the process itself: how do different actors argue, what kind of strategies and rhetorics do they use, to which instruments and institutions do they refer, and in what way do actors react to each other? An analysis of communicative interactions contributes to the question of how international negotiations work. The analytic inclusion of sociolinguistic practices allows insights into positions, strategies, and perspectives pertaining to cultural property. By looking at not only what actors say, but also at how and in what contexts they do so, it is possible to make more accurate statements about their positions and perceptions in cultural property debates. As these communicative interactions influence outcomes considerably, an approach from linguistic anthropology is not only beneficial for an understanding of specific negotiations, but also for the analysis of broader cultural property issues.
\end{abstract}

